DOE/OR/21389--38

DE93 002800

\title{
The Potential for Biomass to Mitigate Greenhouse Gas Emissions in the Northeastern U.S.
}

Stephen S. Bernow

Kevin Gurney

Gary Prince

Tellus Institute

with

Michael Cyr

A Report to
CONEG POLICY RESEARCH CENTER, INC.

Northeast Regional Biomass Program

Tellus Institute

Boston, MA

April 1992

$8 B$ 


\section{DISCLAIMER}

This report was prepared as an account of work sponsored by an agency of the United States Government through the CONEG Policy Research Center, Inc. under Grant No. DE-FG05-830R21389. Neither the CONEG Policy Research Center, Inc., the United States Government nor any agency thereof, nor any of their employees, makes any warranty, express or implied, or assumes any legal liability or responsibility for the accuracy, completeness, or usefulness of any information, apparatus, product, or process disclosed, or represents that its use would not infringe privately owned rights. Reference herein to any specific commercial product, process, or service by trade name, trademark, manufacturer, or otherwise, does not necessarily constitute or imply its endorsement, recommendation, or favoring by the CONEG Policy Research Center, Inc., the United States Government or any agency thereof. The views and opinions of authors expressed herein do not necessarily state or reflect those of the CONEG Policy Research Center, Inc., the United States Government or any agency thereof. 


\section{Table of Contents}

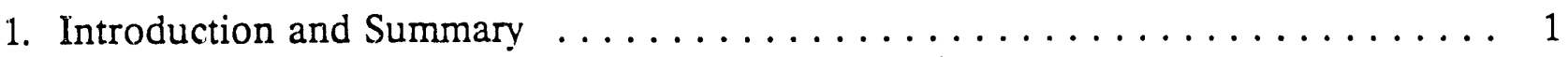

1.1 The Greenhouse Effect and Global Warming $\ldots \ldots \ldots \ldots \ldots \ldots \ldots$

1.2 Greenhouse Gas Policy and the Role of Biomass ............. 2

1.3 Structure and Scope of this Study $\ldots \ldots \ldots \ldots \ldots \ldots \ldots \ldots \ldots$

1.4 Principal Findings of this Study $\ldots \ldots \ldots \ldots \ldots \ldots \ldots \ldots \ldots$

2. Global Warming and the Role of Biomass $\ldots \ldots \ldots \ldots \ldots \ldots \ldots \ldots \ldots$

2.1 The Greenhouse Effect $\ldots \ldots \ldots \ldots \ldots \ldots \ldots \ldots \ldots \ldots \ldots$

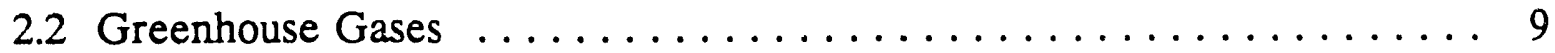

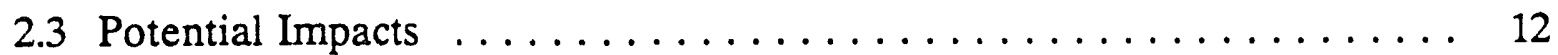

2.4 Policy Responses .............................. 14

2.5 The Biomass Carbon Cycle ..................... 20

2.6 Economic and Environmental Considerations in Biomass Energy Usage ........................... 25

3. CONEG Region Energy and Solid Waste Systems . . . . . . . . . . . 27

3.1 The Current Energy System . . . . . . . . . . . . . . . . . 27

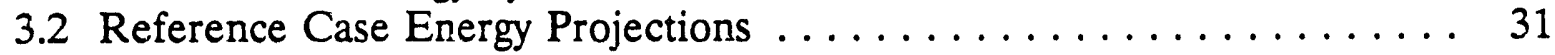

3.3 Conservation Case Energy Projections . . . . . . . . . . . . . 43

3.4 The CONEG States Solid Waste Systems $\ldots \ldots \ldots \ldots \ldots \ldots \ldots \ldots 48$

3.5 Total Emissions: Energy and Solid Waste ............... 52

3.6 Biomass Policy Scenarios . . . . . . . . . . . . . . . . . . . 54

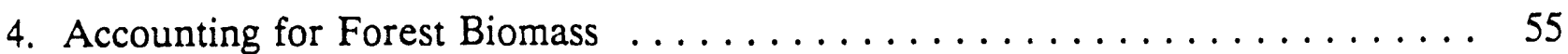

4.1 Definitions and General Procedures $\ldots \ldots \ldots \ldots \ldots \ldots \ldots \ldots \ldots 5$

4.2 Baseline Forest Data . . . . . . . . . . . . . . . . . . 56

4.3 Forest Management ....................... 59

4.4 Forest and Other Land Use Patterns $\ldots \ldots \ldots \ldots \ldots \ldots \ldots \ldots 66$

4.5 Overview of the Forests $\ldots \ldots \ldots \ldots \ldots \ldots \ldots \ldots \ldots \ldots \ldots$

4.6 Forest Products Usage $\ldots \ldots \ldots \ldots \ldots \ldots \ldots \ldots \ldots \ldots \ldots \ldots$

5. Current Biomass Policy: Reference and Conservation Cases . . . . . . . . . . 74

5.1 Current Biomass Policy - Reference Case . . . . . . . . . . . . . 74

5.2 Current Biomass Policy - Conservation Case ............... 81

6. Moderate and Advanced Biomass Policy Scenarios

to Reduce Greenhouse Gases . . . . . . . . . . . . . . . . . . . 86

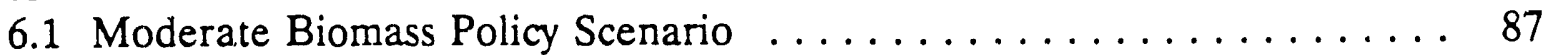

6.2 Advanced Biomass Policy Scenario ...................... 94 
6.3 Solid Waste Policy Results . . . . . . . . . . . . . . . . . . . . 101

6.4 Comparison of Summary Results . . . . . . . . . . . . . . 106

7. Policies for Reducing Greenhouse Gas Emissions $\ldots \ldots \ldots \ldots \ldots \ldots \ldots$

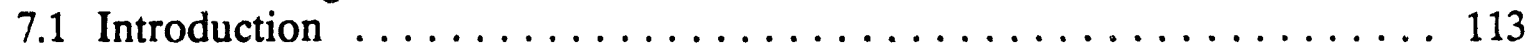

7.2 Policy Recommendations . . . . . . . . . . . . . . . . . 122

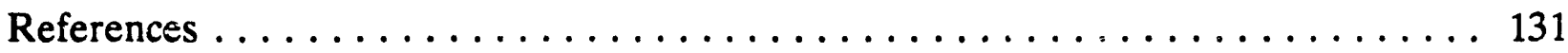

APPENDIX A: CONEG REGION SOI ID WASTE SYSTEM: STATE-SPECIFIC GREENHOUSE GAS EMISSIONS

APPENDIX B: $\quad$ ASSUMPTIONS OF CONEG STATE LAND USE, FOREST BIOMASS, AND WOOD USES

APPENDIX C: SUMMARY DESCRIPTIONS OF CONEG STATE FORESTS AND FOREST PRODUCTS 


\section{List of Tables}

Table $2.1 \quad$ Current Greenhouse Gas Emissions . . . . . . . . . . . . . . . . . 11

Table 2.2 Greenhouse Gas Emission in Global Warming Potential . . . . . . . 12

Table 2.3 International Greenhouse Gas Reduction Protocols . . . . . . . . . . . 15

Table 2.4 State Carbon Dioxide Valuations ....................... 19

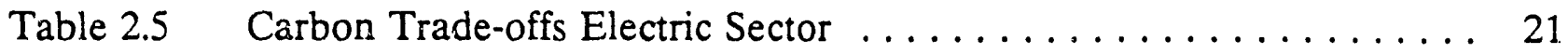

Table 3.1 CONEG Region 1988 End-Use Energy Demands . . . . . . . . . . . . 28

Table 3.2 CONEG Region, 1988 End-Use Renewable Energy Demands . . . . . 29

Table 3.3 CONEG, 1988 Electric Generation Mix . . . . . . . . . . . . . . . 29

Table $3.4 \quad$ CONEG Reference Case 1988 Biomass-Electric Power . . . . . . . . . 30

Table 3.5 CONEG Region, Reference Scenario End-Use Demand Growth

Rates ............................ 33

Table 3.6a Reference Case End-Use Energy Projections

New England ........................ 34

Table 3.6b Reference Case End-Use Energy Projections

Mid-Atlantic ..................... 34

Table 3.6c Reference Case End-Use Energy Projections

New York .......................... 35

Table 3.7 Reference Case Biomass Electric Capacity ............... 36

Table 3.8 Reference Case Electric Generation Projections

New England ....................... 38

Table 3.9 Reference Case Electric Generation Projections

Mid-Atlantic .......................... 39

Table 3.10 Reference Case Electric Generation Projections

New York $42 \ldots \ldots \ldots \ldots \ldots \ldots \ldots \ldots$.............. 40

Table 3.11 Reference Case, CONEG Energy System Greenhouse Gas Emissions . 42

Table 3.12 Conservation Case: End-Use Energy Projections

a,b c ................................. 44

Table 3.13 Conservation Case Electric Generation Projections

New England ....................... 45

Table 3.14 Conservation Case Electric Generation Projections

Mid-Atlantic ....................... 46

Table 3.15 Conservation Case Electric Generation Projections

New York ............................. 47

Table 3.16 Conservation Case, CONEG Energy System

Greenhouse Gas Emissions ................. 48

Table 3.17 CONEG Region Solid Waste System

Greenhouse Gas Emissions ................ 51

Table 3.18 Reference Case Energy and Landfill Emissions . . . . . . . . . . 53

Table 3.19 Conservation Case Energy and Landfill Emissions ............. 54 


\section{List of Tables \\ (Continued)}

Table $4.1 \quad$ Mean Annual Growth $\ldots \ldots \ldots \ldots \ldots \ldots \ldots \ldots \ldots \ldots$

Table 4.2 Estimated Per Acre Yields for Growing Stock ............ 58

Table 4.3 Current Forest Management Practice on

Ail Forest Land FY 1989 . . . . . . . . . . . . . . . . . 61

Table 4.4 Firewood and Commercial Wood Harvesting

Accounts/Losses Assumptions . . . . . . . . . . . . . . . 64

Table 4.5 Commercial Wood Accounts/Loss Assumptions ............. 66

Table 4.6 CONEG States Land Areas, 1000 Acres . . . . . . . . . . . . . . . 67

Table 4.7 Percent Shares of Key Land Types in the CONEG States . . . . . . . 68

Table 4.8 Commercial Forest Ownership Breakdown

Thousands of Acres .................... 70

Table 4.9 Estimated Wood End-Use in the CONEG States . . . . . . . . . 72

Table $5.1 \quad$ Biomass Demand Inputs . . . . . . . . . . . . . . . 76

Table 5.2 Current Policy Wood-Electric Power Supply

in the CONEG Region .................. 77

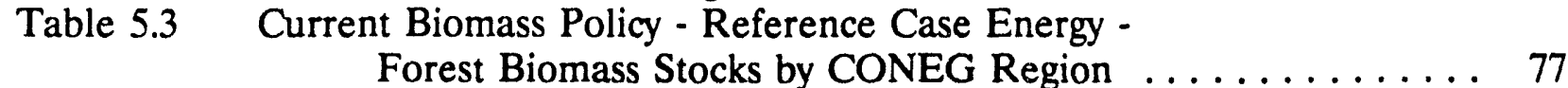

Table 5.4 Comparison of Tellus and R. Seymour Forecasts

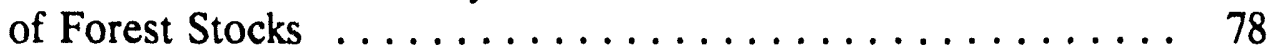

Table 5.5 Current Biomass Policy - Reference Case Energy -

Forest Biomass Yields by CONEG Region ........... 79

Table 5.6 Current Biomass Policy - Reference Case Energy -

Forest Carbon Uptake in the CONEG Region . . . . . . . . 80

Table 5.7 Carbon Balances for the CONEG Region - Current Biomass

Policy - Reference Case Energy . . . . . . . . . . . . 81

Table 5.8 Current Biomass Policy - Conservation Case Energy -

Forest Biomass Stocks by CONEG Region .......... 82

Table 5.9 Current Biomass Policy - Conservation Case Energy -

Forest Biomass Yields by CONEG Region . . . . . . . . . . 83

Table 5.10 Current Biomass Policy - Conservation Case Energy -

Forest Carbon Uptake in the CONEG Region . . . . . . . . 84

Table 5.11 Carbon Balances for the CONEG Region - Current Biomass

Policy - Conservation Case Energy .............. 85 


\section{List of Tables \\ (Continued)}

Table 6.1 Moderate Biomass Policy Wood-Electric Power Supply

in the CONEG Region .................. 87

Table 6.2 Moderate Biomass Policy - Conservation Case Energy -

Forest Biomass Stocks by CONEG Region . . . . . . . . . 90

Table 6.3 Moderate Biomass Policy - Conservation Case Energy -

Forest Biomass Yields by CONEG Region . . . . . . . . . 90

Table 6.4 Moderate Biomass Policy - Conservation Case Energy -

Forest Carbon Uptake in the CONEG Region . . . . . . . . . 92

Table 6.5

Table 6.6

Moderate Biomass Policy Capacity Additions . . . . . . . . . . . . . . 93

Carbon Balances for the CONEG Region - Moderate Biomass

Policy - Conservation Case .................... 94

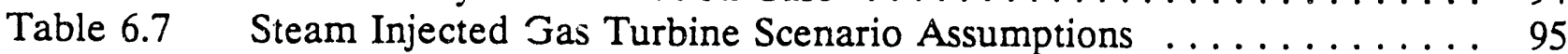

Table 6.8 Advanced Biomass Policy Wood-Electric Power Supply

in the CONEG Region ................... 96

Table 6.9

Additional Land Required for Wood-Electric Power . . . . . . . . . . 97

Table 6.10

Table 6.11

Table 6.12

Table 6.13

Potential Residential Energy Savings From Urban Tree Planting . . . . 99

Total Annual Carbon Reduction Savings From Urban Tree Planting

In the CONEG Region ..................... 100

CONEG Region Solid Waste System Greenhouse Gas Emissions . . . 102 Advanced Biomass Policy - Conservation Case Energy -

Forest Biomass Stocks by CONEG Region ........... 103

Table 6.14 Advanced Biomass Policy - Conservation Case Energy -

Forest Biomass Yields by CONEG Region ............ 104

Table 6.15 Advanced Biomass Policy - Conservation Case Energy -

Forest Carbon Uptake and Release in the CONEG Region ... 105

Table 6.16 Carbon Balances for the CONEG Region - Advanced Biomass

Policy - Conservation Case ..................... 106

Table 6.17 Forest Stocks by CONEG Region for Four Scenarios . . . . . . . . . . . . 108

Table 6.18 Forest Yields by CONEG Region for Four Scenarios . . . . . . . . . . . . . 109

Table 6.19 Net Carbon Uptake by Region for Four Scenarios . . . . . . . . . . . . 110

Table 6.20 Carbon Balances for the CONEG Region Four Scenarios

(Million Tons Carbon Equivalent) .............. 112

Table 7.1 Biomass/Fossil Cost Trade-offs Electric Sector 2000 In-Service Date . . . . . . . . . . . . . . . . . . . . . 119

Table 7.2 Costs of Carbon Dioxide Reduction From Energy Supply and

Demand Options ....................... 120

Table 7.3 Costs of Forestry Options for Carbon Storage/Reduction . . . . . . . . 121 


\section{List of Figures}

Figure $2.1 \quad$ Marginal Cost of Carbon Emission Reductions $\ldots \ldots \ldots \ldots \ldots$

Figure 4.1 Recent Forest Management Practice on All Forested Land in the CONEG States $\ldots \ldots \ldots \ldots \ldots \ldots \ldots \ldots \ldots \ldots \ldots \ldots$ 


\section{Introduction and Summary}

This study, for the Northeast Regional Biomass Program (NRBP) of the Coalition of Northeast Governors (CONEG), evaluates the potential for local, state and regional biomass policies to contribute to an overall energy/biomass strategy for the reduction of greenhouse gas releases in the Northeastern United States. Biomass is a conditionally renewable resource that can play a dual role: by reducing emissions of greenhouse gases in meeting our energy needs; and by removing carbon from the atmosphere and sequestering it in standing biomass stocks and long-lived products.

In this study we examine the contribution of biomass to the energy system in the Northeast and to the region's net releases of carbon dioxide and methane, and project these releases over three decades, given a continuation of current trends and policies. We then compare this Reference Case with three alternative scenarios, assuming successively more aggressive efforts to reduce greenhouse gas emissions through strategic implementation of energy efficiency and biomass resources. Finally, we identify and examine policy options for expanding the role of biomass in the region's energy and greenhouse gas mitigation strategies.

\subsection{The Greenhouse Effect and Global Warming}

In recent years, great attention has been focused on the threat of global warming. Based on models of the atmosphere and other evidence, many scientists have predicted that the buildup of certain anthropogenic air emissions, particularly carbon dioxide, will cause a "greenhouse effect" by which the earth's temperature will increase over the next several decades.

"Global warming" or the "greenhouse effect" are commonly used expressions to describe the anticipated rise in global average temperature owing to continued releases of the greenhouse gases into the earth's atmosphere from human activities. These gases absorb solar energy that has reached the 9arth and is reflected back towards space, and re-radiate some of this energy back down to earth. The earth's temperature will consequently reach a higher equilibrium level. The principal gases of concern carbon dioxide $\left(\mathrm{CO}_{2}\right)$, methane $\left(\mathrm{CH}_{4}\right)$, nitrous oxide $\left(\mathrm{N}_{2} \mathrm{O}\right)$, and the chlorofluorocarbons (CFCs) - are common by-products of industrial activity which have significantly increased above pre-industrial levels since approximately 1850 in both annual release rates and cumulative concentrations in the atmosphere. The combustion of fossil fuels to produce energy is one of the leading sources of greenhouse gas emissions, accounting for 85 percent of the existing global $\mathrm{CO}_{2}$ emissions and about 60 percent of total global warming potentici. of all greenhouse gases from all sources. Among the other sources are: deforestation and combustion or decay of forest biomass, production and use CFCs, cement production, rice cultivation, animal husbandry, and solid waste disposal. 
If these gases are allowed to accumulate unabated in the atmosphere, some projections indicate that the global average surface temperature of the earth may increase by 2 to 5 degrees celsius (C) by the year 2030. Such a temperature increase would result in a number of related impacts under the broader rubric of "climate change," including sea level rise and the inundation of low-lying coastal areas (including the eastern and southern United States), and shifts in agricultural productivity. Potentially severe ecological, economic, public health and political consequences could ensue in various regions of the world.

\subsection{Greenhouse Gas Policy and the Role of Biomass}

The U.S. contributes about 20 percent of the world's $\mathrm{CO}_{2}$ and its overall greenhouse gas global warming potential (GWP). The Northeastern U.S. contributes about 20 percent of this country's total contribution to global $\mathrm{CO}_{2}$ emissions. The U.S. energy sector reisases about 90 percent of the tota! GWP of all U.S. greenhouse gases. Within the energy sector, promising opportunities for significant reductions of U.S. greenhouse gas emissions are in the electric utility sector, which emits about 2 billion tons of $\mathrm{CO}_{2}$ annually from the combustion of fossil fuels, about 40 percent of all carbon dioxide releases in the nation, and about 30 percent of the total GWP carbon equivalent of all greenhouse gases released in the U.S.

Energy efficiency improvements and fuel switching can be important parts of a solution, as these will reduce both the overall level of fuel combustion and the carbon content of the fuels consumed. Renewable sources of energy that do not emit greenhouse gases - solar, geothermal, wind, hydro, etc. - can also play a role, by displacing fossil fuels and thereby helping to reduce carbon dioxide emissions from the energy sector. Biomass is a conditionally renewable resource that removes carbon dioxide from the atmosphere, sequesters it in standing stocks of biomass or in longlived wood products, and releases it upon combustion or decay. Provided it is utilized on a sustainable basis, with regrowth and carbon uptake off-setting combustion and its carbon releases, biomass can play a significant role as part of an overall strategy to mitigate the potential for global warming.

Biomass can play a negative or a positive role in efforts to stabilize or reduce greenhouse gas concentrations in the atmosphere. Carbon in the form of standing stocks of biomass (primarily in forests) can be released when forests are cut down on a non-sustainable basis, for fuel, wood products, and for agriculture and development. Carbon dioxide and methane are released to the atmosphere through combustion or decay of the woody biomass. Combustion can be for energy production, solid waste incineration or land clearing, while decay can occur on the forest flcor, at mills and at landfills. 
On the other hand, if forests are harvested for these products at sustainable levels, regrowth (or replanting) can maintain a carbon budget equilibrium as carbon dioxide is both released into and taken from the atmosphere to form carbon in standing woody biomass. Moreover, if wood is used as a fuel to displace fossil fuels, which release carbon without a compensating carbon uptake, a net reduction of carbon releases can be effected. Finally, increases in the standing forest stocks through improved forest management practices and afforestation projects will also serve to draw down from the carbon stocks accumulating in the atmosphere.

But for biomass there are other dimensions. Along with energy and greenhouse gases there is the usage and destiny of the lands upon which the biomass resource will depend. Forested lands in particular have alternative uses - paper and pulp and wood products, recreation, watershed and soil protection - and embody other values preservation of natural environments and the flora and fauna therein - apart from human uses. Thus energy and greenhouse gas policies and actions are necessarily intertwined with economic and environmental goals and policies. Consequently, it is desirable to integrate these multiple aims into coherent policy initiatives.

\subsection{Structure and Scope of this Study}

This study focuses on the eleven CONEG states, grouped into three sub-areas.

New England

Connecticut, Maine,

Massachusetts, New Hampshire,

Rhod: Island, Vermont
New York

The analysis focused on two of the most important greenhouse gases: carbon dioxide $\left(\mathrm{CO}_{2}\right)$, which contributes over 70 percent of the projected global warming potential of all greenhouse gases annually released; and methane $\left(\mathrm{CH}_{4}\right)$, which contributes an estimated 11 percent. Because a ton of methane has ten times the cumulative warming effect of a ton of carbon in the atmosphere, a conversion factor was used to estimate emissions of both gases in common terms of "global warming potential" or tons of "carbon equivalent".

Using available information about the region's forests and other land-use patterns, its energy and its solid waste systems, Tellus developed a Reference Case which estimated the current level of $\mathrm{CO}_{2}$ and $\mathrm{CH}_{4}$ emissions (and $\mathrm{CO}_{2}$ uptake from forest growth) in the Northeast, and then projected these emission levels over the next 30 years, given a continuation of current biomass policies and energy trends. Next, the Reference Case was modified to incorporate significant but fully cost-effective energy efficiency measures, themselves capable of reducing carbon emissions in the year 2020 by 20-35 percent compared to the original Reference Case. 
it was within the context of this "Conservation Case" background that the potential impact of more aggressive biomass strategies was tested. The analysis focused on wood-burning for electricity generation, with wood primarily displacing coal-fired plants. The transportation sector was not considered in this analysis, because it was assumed that the biomass grown in the Northeast is less likely than other regions to serve as a major feedstock for transportation fuels in the near future. Carbon equivalent emissions were projected over a 30 -year period under each of four scenarios:

1) Current Biomass Policy - Reference Case Energy Assumptions

2) Current Biomass Policy - Conservation Case Energy Assumptions

3) Moderate Biomass Policy - Conservation Case Energy Assumptions

4) Advanced Biomass Policy - Conservation Case Energy Assumptions

Current Biomass Policy starts with the existing forest biomass resource, assuming no significant changes in forest management practices and a doubling of wood-electric power supply over the next 30 years, from $806 \mathrm{MW}$ in 1988 to $1,676 \mathrm{MW}$ in 2020 . These assumptions are applied first to the Reference Case and then to the Conservation Case, producing the first two scenarios. In the third and fourth scenarios, the demand for end-use energy supply is held constant, while the percentage contribution of biomass to the fuel mix is successively increased.

Moderate Biomass Policy assumes that current and new biomass resources are utilized more fully to supply greater wood-electric energy production $(3,416 \mathrm{MW}$ by 2020), given existing fuelwood combustion technologies. Specific elements of this strategy include:

- $\quad$ increased forest biomass management;

- increased harvest and milling residue usage; and

- increased wood-electric power supply, using existing technology.

Advanced Biomass Policy assumes a still more significant increase in woodelectric supply (up to 6,020 MW by the year 2020), given the development of more efficient combustion technology - the biomass gasification steam injected gas turbine, or BIG STIG. In this scenario:

- forest management and biomass residue use are increased still further;

- $\quad$ BIG STIG technology supplements conventional wood power supply;

- $\quad$ short rotation high yield biomass energy crops are introduced as part of the fuel supply; 
- urban tree planting results in lower demand for building space conditioning and increased carbon uptake and sequestration; and

- greater levels of source reduction, recycling and composting reduce methane releases.

The Long-Range Energy Alternatives Planning (LEAP) system was used to provide the organizing analytical framework for the energy, emissions, and landuse/biomass scenarios. LEAP was linked with its Environmental Data Base (EDB) to provide the specific emission coefficients at each point in the energy system. LEAP and EDB have been developed by the Stockholm Environment Institute - Boston Center (SEI-B) at Tellus Institute, and applied in numerous countries and regions throughout the world. It is a "bottom-up" modelling system whose principal elements are the energy and technology characteristics of sectoral end-uses and supply sources, and the characteristics of land-use and biomass production. ${ }^{1}$

This report is organized into the following sections:

Section 2 summarizes the greenhouse effect, global warming, and the current and potential role of biomass in affecting greenhouse gas emissions.

Section 3 describes the region's current energy and solid waste systems, and projects carbon and methane emissions associated with these systems over a 30-year period, given: a) current energy trends and policies (Reference Case); and b) the achievement of cost-effective energy efficiency goals (Conservation Case).

Section 4 provides information on forest statistics, and the methods and assumptions used in this study to estimate forest biomass and its impact on the region's carbon cycle.

Section 5 combines the carbon cycle impacts associated with Current Biomass Policy with the energy and solid waste systems impacts of

\footnotetext{
${ }^{1}$ LEAP is comprised of a set of flexible computer modules covering demand (sector/sub-sector/enduse/technology and fuel), transformation (electricity supply system and other sources of energy supply), and resources (land-use and biomass production). EDB is a computerized database of energy-related environmental impacts (coefficients for air and water pollutant emissions, land-use impacts, solid waste generation, and on-site health impacts). LEAP development was originally undertaken for the Beijer Institute for Energy and Human Ecology of the Royal Swedish Academy of Sciences, funded by the Swedish International Development Agency (SIDA). EDB was originally funded by the United Nations Development Programme (UNEP), which administers this database along with SEI-B at Tellus. LEAP is administered by SEI-B at Tellus.
} 
the Reference Case and the Conservation Case, to produce Scenarios 1 and 2.

Section 6 applies first Moderate Biomass Policy and then Advanced Biomass Policy to the energy and solid waste system impacts associated with the Conservation Case, resulting in Scenarios 3 and 4.

Section 7 outlines policy options to meet the region's energy, economic, environmental and greenhouse gas goals.

\subsection{Principal Findings of this Study}

This study shows that increased reliance on biomass for energy depends on strengthening afforestation and forest management efforts, including some urban forestry and short rotation crop production in addition to better management of existing
forests.

- Currently, gross carbon equivalent emissions in the region from energy and solid waste is about 314.7 million tons. Net carbon uptake from the region's forests offsets this by 25.6 million tons or about 8 percent, to 289.1 million tons.

- $\quad$ Given current biomass and energy policies, the region's net carbon equivalent emissions would increase from 289.1 million tons in 1988 to 361.7 million tons in 2020, an increase of 25 percent. Forest growth, currently absorbing about 8 percent of gross carbon equivalent emissions from energy use and solid waste, would in this Reference Case absorb only about 7 percent of projected emissions by the year 2020 .

- $\quad$ Aggressive but realistic biomass and energy policies could reduce net carbon equivalenc emissions from 289.1 million tons to 226.9 million tons by the year 2020 - a reduction of 22 percent as compared with an increase of 25 percent in the Reference Case - with forest growth absorbing 15 percent of gross annual emissions by the year 2020 .

- In this "Advanced Biomass Policy" scenario, the annual absorption of carbon by the region's growing forests increases substantially, from 25.6 million tons per year in 1988 to 40.2 million tons per year in 2020 .

The three key areas identified here for expanded biomass strategic and policy efforts are as follows:

(1) Increased use of biomass for electric generation: 
- $\quad$ expanded use of conventional wood electric power supply

- new biomass gasification steam injection turbines (BIG STIGs)

(2) Increased use of advanced solid waste management practices, including:

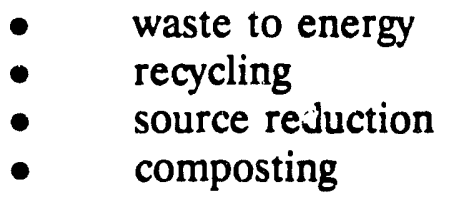

(3) Forestry:

- $\quad$ increased forest management

- $\quad$ increased use of forest biomass residues

- use of short rotation woody crops for biomass energy

- $\quad$ planting urban trees for reducing space conditioning energy requirements, and sequestering carbon

Although conservation has the dominant role to play in reducing the region's emissions of carbon dioxide, biomass strategies could account for about a 4.7 percent additional reduction in net carbon emissions by the year 2020. The region's forests are capable of playing a much greater role in producing energy, as well as other, durable forest products. By creating a reliable market for otherwise less valuable wood, biomass energy production tends to encourage better management of existing forest lands, many of which are dispersed among small private owners.

An effective greenhouse gas emission strategy will need to be built from myriad elements, each contributing a part of the entire production. In this context, biomass strategies and policies can play an important role, and should be considered as part of the region's contribution to a global solution. 


\section{Global Warming and the Role of Biomass}

Recently, great attention has been focused on the threat of global warming. Based on models of the atmosphere, and other evidence, many scientists predict that the buildup of certain anthropogenic air emissions will exacerbate the r aturallyoccurring "greenhouse effect", thereby causing the earth's temperature to increase, with potentially disastrous effects. Among the potential impacts of global warming are: rising sea levels, with resultant flooding of low-lying coastal areas (stch as the eastern and southern United States); changes in rainfall distributions (with concomitant impacts on agriculture); increasing volatility of weather patterns; and the risk that existing animal populations and ecosystems will be unable to migraie or adapt fast enough to adjust to a climate change of unprecedented rapidity.

Both the nature of the causes - chiefly the emission of carbon dioxide $\left(\mathrm{CO}_{2}\right)$ methane $\left(\mathrm{CH}_{4}\right)$, nitrous oxide $\left(\mathrm{N}_{2} \mathrm{O}\right)$ and the chlorofluorocarbons (CFCs) - and the nature of the potential consequences make the threat of global warming a problem of global scope. At this time, there is much international interest in developing protocols for reducing greenhouse gas emissions. Several such protocols have been developed, and som.e nations, states, and even cities have taken actions to reduce greenhouse gas emissions.

This section provides some additional background for understanding the greenhouse gas effect, its causes and potential impacts, as well as the range of policy responses that have been adopted or proposed to date. The second part of this section introduces the role of biomass in the carbon cycle and the relative carbon dioxide emissions characteristics of biomass versus various fossil fuels, and concludes with a brief discussion of Municipal Solid Waste and the emissions associated with different means of managing the solid waste stream.

\subsection{The Greenhouse Effect}

The greenhouse effect itself is a relatively well-understood natural phenomenon; it was first mentioned as early as 1827 in a paper by the physicist-mathematician Jean-Baptiste Fourier. The earth receives a constant amount of energy from the sun mostly in the form of visible or "incoming" radiation. While some of this energy is reflected directly back into space by the atmosphere and the surface of the earth, the majority (approximately 70 percent) is absorbed by the various media comprising the earth's surface and the atmosphere, thereby raising their temperature. This same amount of energy is re-emitted to space as thermal or "outgoing" radiation, thus maintaining an energy balance; the amount of energy entering the earth/atmosphere system is equal to the amount leaving. 
The essence of the greenhouse effect is the ability of particular trace gases in the atmosphere to absorb some of the outgoing radiation on its way out into space from the surface of the earth, thereby raising the temperature of the earth/atmosphere system above what it would be without the presence of those gases. It is this "blanketing" effect of the atmosphere that is referred to as the greenhouse effect. Without the presence of the atmosphere and its trace gases, the global average surface temperature of the earth would be approximately $43^{\circ} \mathrm{C}$ cooler, and under such conditions, life on the planet might have evolved in a significantly different manner. A greenhouse is a useful analogy; the atmosphere behaves somewhat like the glass pane of a greenhouse, letting in visible or shortwave radiation, but impeding somewhat the exit of thermal energy, thereby increasing temperature inside the greenhouse structure.

The present concern centers not on the "natural" greenhouse effect of the atmosphere on global equilibrium temperature (and climate), under which life as we know it has evolved, but on the potential additional global warming that may occur due to increases in the gases responsible for absorbing the released outgoing radiation.

\subsection{Greenhouse Ga:es}

The principal gases responsible for absorbing the outgoing radiation in order of present expected impact in the atmosphere are: carbon dioxide, methane, chlorofluorocarbons, and nitrous oxide. Carbon monoxide eventually becomes $\mathrm{CO}_{2}$ when residing in the atmosphere. Measurements taken at various remote locations around the globe have revealed that these gases are substantially exceeding pre-industrial levels. Primarily responsible for this growth in atmospheric concentrations are the dual effects of increasing population and increased combustion of fossil fuels. The following examines the principal sources and sinks (absorption and storage sites) of these gases in addition to their present growth characteristics.

\section{Carbon Dioxide}

The present emissions of carbon dioxide currently contribute more than 70 percent of the projected global warming potential of all greenhouse gases annually released. The primary sources of carbon dioxide emissions into the atmosphere are the production of commercial energy, tropical deforestation, and miscellaneous (cement manufacturing, oxidation of carbon monoxide, etc.) sources. The concentration of carbon dioxide in the atmosphere is presently 355 parts per million (ppm) increasing at a rate of 0.4 percent annually. Pre-industrial levels were about $280 \mathrm{ppm}$. Once emitted, carbon dioxide cycles through the atmosphere and the biota before being permanently removed by oceanic processes or long term increases in terrestrial biotic storage. The effective residence time of carbon dioxide in the atmosphere before permanent removal is 50 to 200 years. 


\section{Methane}

The present emissions of methane have been estimated to contribute about 11 percent of the projected global warming potential of all greenhouse gases annually released. Methane has both natural and anthropogenic sources: the latter primarily consists of releases from fuel production, enteric fermentation in ruminants, rice cultivation, landfill emissions, and deforestation. The present concentration of metiane in the atmosphere is $1.7 \mathrm{ppm}$, and is growing at about 0.9 percent per year. The estimated residence time of methane in the atmosphere is approximately 12.5 years. Methane is principally removed from the atmosphere by reacting with the hydroxyl radical $(\mathrm{OH})$. Because reaction with $\mathrm{OH}$ is the main chemical removal process in the atmosphere, increasing methane concentrations can have significant effects on the general ability of the atmosphere to remove other greenhouse gases.

\section{Chlorofluorocarbons}

The present emissions of chlorofluorocarbons (CFC) have been estimated to contribute about 7 percent of the projected global warming potential of all greenhouse gases annually released. Though existing in relatively trace amounts in the atmosphere, this class of chemical compounds exhibits powerful radiative trapping abilities in addition to their well-known ozone depleting properties. CFCs are solely of human origin, used in various industrial processes and end-use products ranging from fog horns to refrigerator insulation. The present concentration of two of the main CFCs, CFC-11 and CFC-12, are 280 and 484 parts per trillion (ppt), respectively, and are exhibiting relatively large growth rates of approximately 4 percent per year. They are removed from the atmosphere through reaction with $\mathrm{OH}$ or more commonly by reacting with sunlight. Residence times in the atmosphere range everywhere from a few years to 400 years depending on the particular CFC.

\section{Nitrous Oxide}

The present emissions of nitrous oxide have been estimated to contribute about 2 percent of the projected global warming potential of all greenhouse gases annually released. While having many natural sources, the use of fertilizer and combustion account for the majority of anthropogenic emission sources. The present concentration of nitrous oxide in the atmosphere is 310 parts per billion (ppb), and is growing at a rate of about 0.25 percent per year. The residence time of nitrous oxide in the atmosphere is estimated to be approximately 150 years.

Table 2.1 summarizes global, U.S., and CONEG region greenhouse gas emissions from essentially all sources, including energy extraction, processing, delivery and usage, cement production, agriculture, livestock, and biomass decay or combustion 
from land conversions. ${ }^{2}$ The world and U.S. emissions are taken from research at the Stockholm Environment Institute-Boston Center at Tellus Institute (SEI, 1991), while the CONEG region emissions are taken from research sponsored by the U.S. EPA (EPA, 1990). ${ }^{3}$ (Later the $\mathrm{CO}_{2}$ and $\mathrm{CH}_{4}$ figures will be compared with the results of this study.)

Table 2.1

Current Greenhouse Gas Emissions

(Million Tons)

\begin{tabular}{lccccc} 
& $\mathrm{CO}_{2}$ & $\mathrm{CO}$ & $\mathrm{CH}_{4}$ & $\mathrm{~N}_{2} \mathrm{O}$ & $\mathrm{CFCs}$ \\
World & 26,092 & 1,224 & 387 & 4.16 & 2.26 \\
U.S.A. & 5,046 & 149 & 36 & 0.83 & 0.65 \\
CONEG & 850 & $9^{4}$ & 6 & $\cdots$ & 0.10 \\
& & & & & \\
\hline
\end{tabular}

Since each of these radiatively active gases contribute to different degrees to potential cumulative global warming, depending upon their instantaneous radiative forcing per unit of gas and their residence times in the atmosphere, global warming potentials (GWPs) relative to $\mathrm{CO}_{2}$ have been proposed by a number of researchers. Here, for illustrative purposes, we use those developed by Lashof and Ahuja (1990) to provide the emissions in carbon dioxide global warming equivalents in Table 2.2. Thus, for example, over the long term a ton of methane has ten times the cumulative warming effect of a ton of carbon dioxide.

\footnotetext{
${ }^{2}$ In the U.S. there is actually a ne increase in standing biomass from forest growth.

${ }^{3}$ The EPA document also gives U.S. totals at 5,517 million tons, which is about 9 percent higher than the SEI number given above, but since it does not take account of biomass growth, it is only 5 percent higher on a gross carbon dioxide release basis. Oak Ridge National Laboratory (ORNL 1990) gives world and U.S. carbon dioxide releases at 23,768 and 5,284 million tons, respectively.

4 The U.S. total for CO in the EPA report is about 40 percent of the U.S. total in the SEI study. Thus we could expect that, consistent with the SEI total for the U.S. given above, the CONEG total would be about 22 million tons.
} 
Table 2.2

Greenhouse Gas Emission in Global Warming Potential

(Million Tons $\mathrm{CO}_{2}$ Equivalent)

\begin{tabular}{|c|c|c|c|c|c|c|}
\hline & $\mathrm{CO}_{2}$ & $\mathrm{CO}$ & $\mathrm{CH}_{4}$ & $\mathrm{~N}_{2} \mathrm{O}$ & CFCs & Total \\
\hline GWP & 1.0 & 2.2 & 10.0 & 180 & 1152 & - \\
\hline $\begin{array}{l}\text { World } \\
\text { U.S.A. } \\
\text { CONEG }\end{array}$ & $\begin{array}{r}26,092 \\
5,046 \\
850\end{array}$ & $\begin{array}{r}2,693 \\
328 \\
20^{5}\end{array}$ & $\begin{array}{r}3,870 \\
363 \\
56\end{array}$ & $\begin{array}{r}748 \\
148 \\
\cdots--\end{array}$ & $\begin{array}{r}2,603 \\
749 \\
115\end{array}$ & $\begin{array}{r}36,006 \\
6,634 \\
1,041\end{array}$ \\
\hline
\end{tabular}

Note that the U.S. contributes about 20 percent to the world's annual $\mathrm{CO}_{2}$ releases, while the CONEG region contributes about 17 percent of the U.S. total $\mathrm{CO}_{2}$ emissions. ${ }^{6}$ In the U.S. and the CONEG region, the electric utility sector contributes about 40 nercent to total $\mathrm{CO}_{2}$ emissions and very small amounts to emissions of the other greenhouse gases.

\subsection{Potential limpacts}

In order to estimate what future changes in climate might occur as a result of greenhouse gas increases, models of climate, called general circulation models (GCM's), have been developed with various assumptions about the workings of the physical climate. Although uncertain, the near-consensus projected global average temperature rise for a doubling of pre-industrial carbon dioxide-equivalent is about 2 to 5 degrees $\mathrm{C}$, expected to occur by the year 2030 under present trends. Regionally, this could be as much as $10^{\circ} \mathrm{C}$ in the polar regions and as little as $0{ }^{\circ} \mathrm{C}$ in equatorial areas. Warming beyond this estimate is pcssible given further increases in greenhouse gas concentrations.

Aside from changes in global average temperature, a variety of other climate variables, although bracketed in uncertainty, may change as a result of the increased absorption of outgoing radiation and the subsequent rise in temperature. The most important changes are: an increase in global precipitation of up to 15 percent,

\footnotetext{
${ }^{5}$ The U.S. total for CO in the EPA report is about 40 percent of the U.S. total in the SEI study. Thus we could expect that, consistent with the SEI total for the U.S. given above, the CONEG region total would be about 50 million tons.

- As will be seen later, in this study CONEG region $\mathrm{CO}_{2}$ emissions are higher (about 20 percent of the U.S. total) since we have included emissions from outside the region that occur because of energy demands within the region.
} 
especially in mid-latitude regions in winter, decrease in soil moisture over the midlatitudes in summer, increase in soil moisture in high latitudes in winter, diminishing global sea ice and snow cover, an increase in tropical storm intensity, and a rise in global sea level of up to 1 meter for a doubling of dioxide-equivalent from pre-industrial levels.

Linked to these alterations in the climate system are a host of alterations to ecological, biogeochemical, human and animal systems. These responses may occur as a result of the absolute magnitude of climate change and/or the rapidity with which the projected changes are anticipated to occur. Climate change at this magnitude and pace is unprecedented in human history.

A rising sea level due to thermal expansion of the ocean column may raise one of the greatest threats to human populations globally. A 1 meter rise in sea level for example, would flood 7 percent of Bangladesh and 12 percent of Egypt, causing relocation of 5 percent and 14 percent of the population in those countries, respectively. In addition, low-lying delta regions commonly used for agricultural activity in the developing world may be inundated. Storm surge conditions will cause unprecedented tide levels incurring both damage and mitigation costs to low-lying coastal urban areas such as the eastern U.S. Wetlands, crucial as nursery grounds for many commiercially viable fish and as pollution protection mechanisms, may be forced to migrate, changing locatiou as water levels vary, at an unprecedented rate. Where coastal wetlands border areas of human habitation, loss will likely occur.

With a change in climate will come an adjustment in the boundaries and extent of ecological regimes. Areas not constrained by human or natural barriers will likely migrate. Particularly sensitive might be existing biological reserves which lack the necessary size and migration corridors required to facilitate dispersal as habitats change with warming. Biodiversity of these regions may diminish, resulting in loss of species and population control problems. Temperate and boreal forests may not migrate with sufficient speed, restilting in forest and habitat loss. The resulting impacts to human populations will be greatest in the developing world where societies and related economies are closely dependent on natural terrestrial ecosystems for their welfare.

The relationship of human health to water availability and quality, and to food availability, raises the likelihood of significant human health impacts from global warming. In addition, a warmer climate may favor vector borne diseases, such as yellow fever, dengue fever and malaria, allowing them to spread towards the poles.

While climate inodels indicate a generally warmer world, regions exist in which water availability may decrease. Existing water resource systems may lack flexibility in dealing with significant changes in the hydrological regime in a given area. Many areas of the globe projected to experience water deficiencies are already experiencing water availability problems. The greatest impact will be on the agricultural sector which in the 
U.S. claims 83 percent of the annual streamflow and has 13 percent of its agricultural land under irrigation.

The world presently exhibits regions of food deficiency resulting, in many instances, from distribution inadequacies. Given the projections of soil moisture and precipitation, food producing regions of the globe will meet with unfavorable climatic conditions which may result in increased malnutrition and starvation to the populations of food importing countries. One of the largest grain producers, the U.S. agricultural industry, has matured under relatively favorable climatic conditions. Genetically engineered species which respond favorably to existing climatic conditions, but exhibit little tolerance to variations in climate, dominate the staple crops in the U.S.

Exacerbation of the existing stress coupled with the lack of flexibility in the major food producing regions may pose the greatest threat to human populations from global warming.

Specific local impacts in the Northeastern U.S. could include coastal inundation by ocean waters from a general sea-level rise, increased severity of storms, and decline in the region's current forests. These can ultimately have direct economic, infrastructural, ecological, recreational, and human health and safety impacts. Beyond these concerns, however, the citizens and policy makers in the region have a potential interest in impacts far beyond the region, in the U.S. and the rest of the world, both in terms of its own economic and environmental security and as an ethical commitment to other peoples, future generations, and the environmental integrity of the planet itself.

\subsection{Policy Responses}

Several international organizations have attempted to establish guidelines or targets for $\mathrm{CO}_{2}$ emissions reductions based on atmospheric stabilization or acceptable levels of climate change. For instance, Working Group 1 of the Intergovernmental Panel Climate Change has "calculate[d] with confidence that... the long-lived gases [e.g., $\mathrm{CO}_{2}$ ] would require immediate reductions in emissions of over 60 percent to stabilize their concentrations at today's level."

Many major industrialized countries have established targets for reducing $\mathrm{CO}_{2}$ emissions, as shown in Table 2.3. These targets, while not sufficient to meet the longterm stabilization goals, are considered starting points intended to curb what might otherwise be rather substantial increases in $\mathrm{CO}_{2}$ in the near term. Even achieving a zero reduction, or no increase in global emissions, will require governmental actions in most countries, including the United States. 


\section{Unilateral Efforts to Reduce Greenhouse Gases}

\begin{tabular}{|c|c|}
\hline AUSTRALIA & $\begin{array}{l}\text { - in October 1990, agreed to a } 20 \text { percent cut in gases not controlled by the } \\
\text { Montreal Protocol, from } 1998 \text { levels by } 2005\end{array}$ \\
\hline AUSTRIA & - agreed to a 20 percent cut in $\mathrm{CO}_{2}$ from 1990 levels by 2005 \\
\hline DENMARK & - agreed to a 20 percent cut in $\mathrm{CO}_{2}$ from 1990 levels by 2000 \\
\hline \multirow[t]{2}{*}{ GERMANY } & - in June 1990, agreed to a $25-30$ percent cut in $\mathrm{CO}_{2}$ from 1987 levels by 2005 \\
\hline & $\begin{array}{l}\text { - in October 1990, the Bundestag released a report outlining specific programs in } \\
\text { each sector to curb greenhouse gas emissions }\end{array}$ \\
\hline ITALY & $\begin{array}{l}\text { Environment Minister Giorgio Ruffolo committed to a } 20 \text { percent cut in } \mathrm{CO}_{2} \text { by } \\
2005\end{array}$ \\
\hline LUXEMBOURG & - agreed to a 20 percent cut in $\mathrm{CO}_{2}$ from 1990 levels by 2005 \\
\hline NETHERLANDS & $\begin{array}{l}\text { - in May 1990, agreed to a } 3-5 \text { percent annual cut in } \mathrm{CO}_{2} \text { from } 1989-90 \text { levels by } \\
2000\end{array}$ \\
\hline \multirow[t]{2}{*}{ NEW ZEALAND } & $\begin{array}{l}\text { - in October } 1990, \text { announced a five-year acceleration of previous government's } \\
\text { commitment to } \mathrm{CO}_{2} \text { reductions, agreeing to a } 20 \text { percent cut from } 1990 \text { levels by } \\
2000\end{array}$ \\
\hline & - a list of specific proposals is to be sent to the Cabinet by mid-1991 \\
\hline NORWAY & $\begin{array}{l}\text { the Parliament approved a carbon tax effective January } 1,1991 \text { (oil is taxed at US } \\
\$ .05 / \text { liter; gasoline at } \$ .10 / \text { liter; natural gas at } \$ .10 / \text { cubic meter; coal is exempt) }\end{array}$ \\
\hline \multicolumn{2}{|r|}{ Unilateral Efforts to Stabilize Greenhouse Gases } \\
\hline AUSTRALIA & $\begin{array}{l}\text { - in October } 1990 \text {, agreed to stabilize gases not controlled by ine Montreal Protocol } \\
\text { at } 1988 \text { levels by } 2000\end{array}$ \\
\hline BELGIUM & - agreed to a 5 percent cut in $\mathrm{CO}_{2}$ by 2000 , and "substantial reductions" thereafter \\
\hline CANADA & $\begin{array}{l}\text { - in December } 1990, \text { agreed to stabilize } \mathrm{CO}_{2} \text { and other greenhouse gases at } 1990 \\
\text { levels by } 2000\end{array}$ \\
\hline \multirow[t]{2}{*}{$\begin{array}{l}\text { EUROPEAN } \\
\text { COMMUNITY }\end{array}$} & $\begin{array}{l}\text { - in October } 1990, \text { energy and environment ministers agreed to a community-wide } \\
\text { stabilization of } \mathrm{CO}_{2} \text { at } 1990 \text { levels by } 2000\end{array}$ \\
\hline & - the Council of Ministers is currently considering policies to achieve cuts \\
\hline $\begin{array}{l}\text { EUROPE FREE } \\
\text { TRADE AGREEMENT }\end{array}$ & $\begin{array}{l}\text { - in November 1990, agreed to adopt the same overall } \mathrm{CO}_{2} \text { target as the European } \\
\text { Community }\end{array}$ \\
\hline \multirow[t]{2}{*}{ JAPAN } & - in June 1990 , agreed to stabilize $\mathrm{CO}_{2}$ on a per capita basis at 1990 levels by 2000 \\
\hline & - in October 1990, agreed to stabilize all greenhouse gases at 1990 levels by 2000 \\
\hline NETHERLANDS & $\begin{array}{l}\text { - agreed to stabilize } \mathrm{CO}_{2} \text { at } 1988-1989 \text { levels by } 1994-1995 \text {, as part of a national } \\
\text { environmental policy plan }\end{array}$ \\
\hline UNITED KINGDOM & - called for stabilization of $\mathrm{CO}_{2}$ at 1990 levels by 2005 \\
\hline
\end{tabular}

Source: The Bureau of National Affairs Inc., Washington D.C., February 27, 1991. 
Stricter emission reduction targets will need to be implemented soon to avoid unacceptable levels of climate change over the longer term. We should anticipaie targets on the order of 30 to 60 percent of current $\mathrm{CO}_{2}$ emissions, sometime witisin the next 40 years, e.g., 30 percent by 2010 to 2015 and 50 percent by 2030 . Accordingly, we must begin to plan for and implement the elements for achieving those targets today. Significant global warming will likely occur as a result of current and near term gree!house gas emissions, and actions to reduce these emissions are important in helping to realize climate stabilization goals.

A $\mathrm{CO}_{2}$ reduction cost curve developed by Nordhaus based upon his survey of $\mathrm{CO}_{2}$ studies (1990) indicates that a $\mathrm{CO}_{2}$ reduction of 20 percent could be achieved at a marginal cost of about $\$ 15$ per ton of $\mathrm{CO}_{2}$, and that a reduction of 30 percent would have a marginal cost of about $\$ 25$ per ton. A reduction of 50 percent would, according to Nordhaus' analysis, have a marginal cost of more than $\$ 50$ per ton.'

Numerous cost analyses of options for reducing carbon emissions in OECD countries give a range of results, largeiy because of contrasting methodological approaches, study assumptions, and national situations. As Grubb has pointed out, "Lovins claims that US consumption of oil and electricity can be reduced by 75 percent, saving trillions of dollars in the process, while Manne and Richeis estimate that merely constraining carbon emissions to present levels could cost the US trillions."

Figure 2.1 illustrates some of these differing results, which differ by a factor of three or four for a given level of emissions reductions. For instance, the marginal cost per ton of carbon dioxide for an approximately 30 percent reduction range from under $\$ 50$ to over $\$ 150$. An EEC study for Italy found that a 10 percent emissions reduction would have a marginal cost of more than $\$ 400$ per ton of $\mathrm{CO}_{2}$.

7 The relationship between marginal cost and percentage reduction developed by Nordhaus is based upon a survey which included cost data from nine different studies. Nordhaus fits a regression line to the results of these studies. Two of these studies -- representing over 50 percent of the data points analyzed -were studies by Nordhaus himself.

8 Grubb's comments include identification of weakness in current macroeconomic modelling of energy use, including the assumption that energy markets behave optimally (Grubb, 1990). 


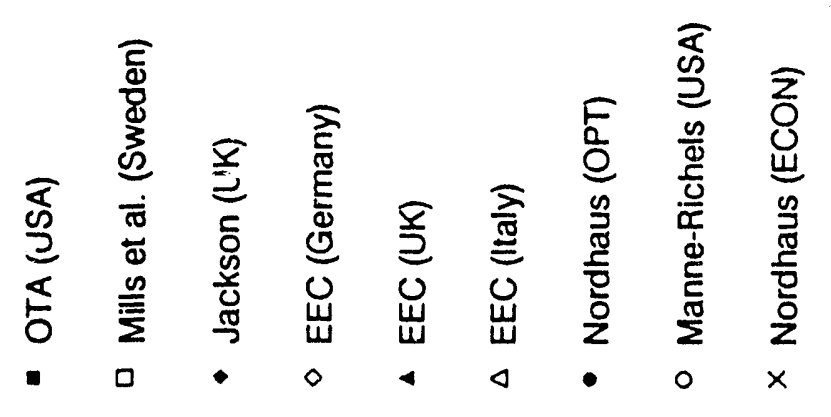

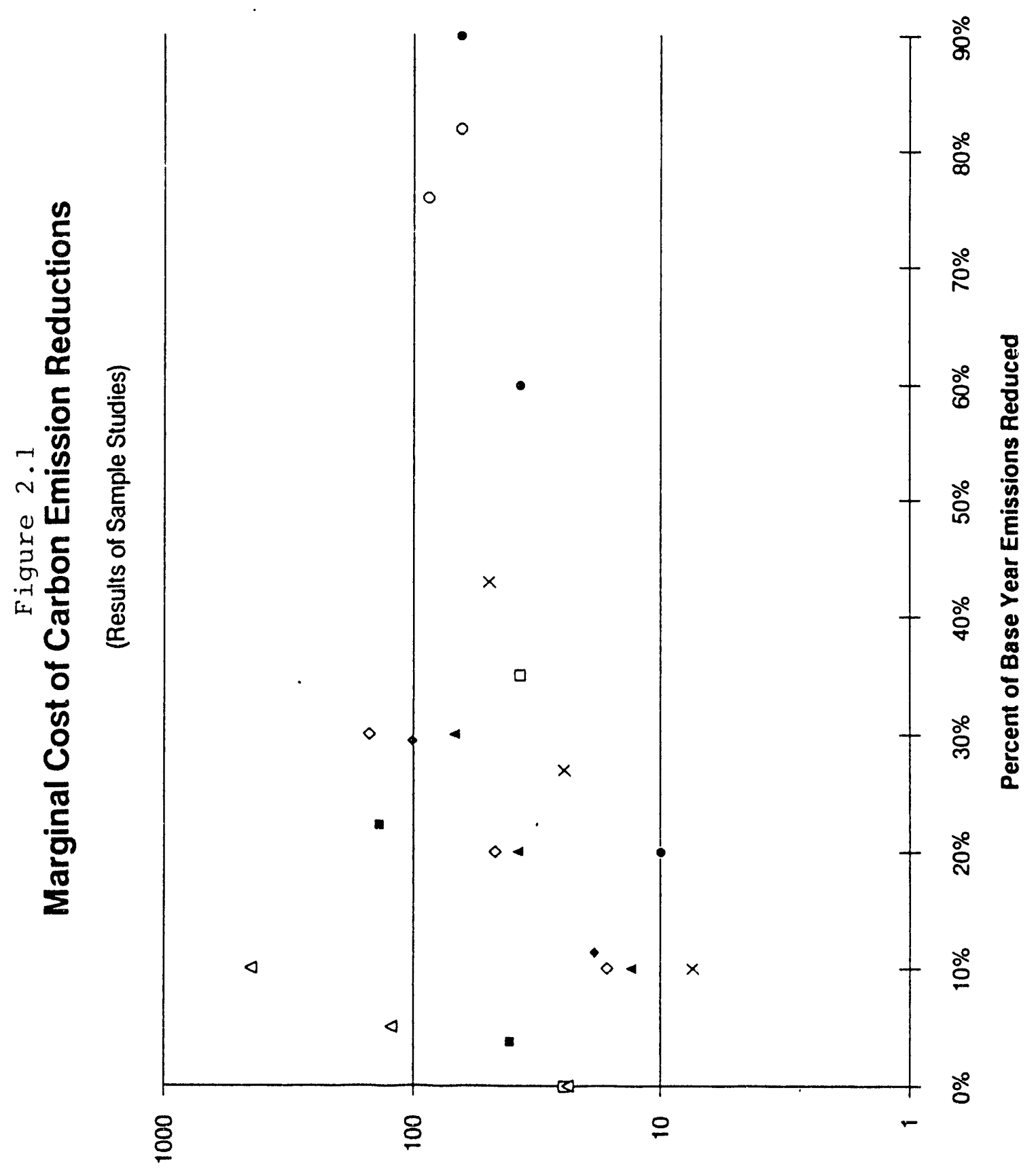

pasnpay ap!xo! u voquoj u0! Hous 120 \$ $\$ 866$ 
Such cross-comparisons exhibit differences owing to different assumptions, including: discount rates, assumed levels of conservation that would occur without intervention, the time period considered, and assumed economic growth rates and future fuel prices, just to name a few.' Nonetheless, such comparison, if interpreted with caution, can help to determine the magnitude of the marginal cost associated with various levels of reduction. While we should interpret these studies with caution, these results provide a useful indication of the magnitude of costs that would be required to achieve significant reductions in greenhouse gases and stabilization of global climate.

Recently, Tellus Institute, in collaboration with Union of Concerned Scientists, American Council for an Energy Efficient Economy, Alliance to Save Energy, and Natural Resources Defense Council, completed a study which indicated that net carbon dioxide reductions approaching 25 percent by 2005 and 70 percent by 2030 could be realized by a variety of actions and policies, at a marginal cost of up to about $\$ 25$ per ton. The Oregon Department of Energy found that options up to $\$ 40$ per ton on the power supply side and about $\$ 21$ to $\$ 66$ per ton for planting or replanting new forests would be needed to reach significant carbon reduction goals.

Currently, there are no existing national regulations explicitly governing releases of carbon dioxide. Scientists throughout the world have identified the risks of potentially catastrophic consequences, and policy makers appear to be moving towards an international protocol for greenhouse gas limitations, and some countries have begun to adopt $\mathrm{CO}_{2}$ targets and/or taxes to realize climate stabilization goals. Some states in the U.S. have adopted carbon dioxide cost adders for resource acquisition. Table 2.4 on the following page summarizes valuations for carbon duxide for energy planning which have been proposed or adopted in various states.

The costs of various options for reducing carbon emissions include those of energy efficiency, fuel switching, power supply alternatives, and afforestation. The Department of Public Utilities in Massachusetts has adopted a $\mathrm{CO}_{2}$ cost of $\$ 23$ per ton emitted - for purposes of utilities' resource planning, evaluation and acquisition (Massachusetts DPU, 1990) - based upon modest levels of afforestation. As greater levels of afforestation are pursued the land and other costs will likely increase well above the value used here. Alternative costs for carbon removal, in the neighborhood of $\$ 20$ to $\$ 30$ per ton removed, could be c'eveloped from power plant carbon removal technologies. The

\footnotetext{
9 An additional reason for inconsistencies relates to study methodology. In part this divergence reflects the split between the two major camps in energy/economic approaches and modelling: the engineeringeconomic approach and associated "bottom-up" models and the macroeconomic approach and its "topdown" models. The bottom-up analysts typically find that cost-effective efficiency measures (and some fuel switching) can reduce carbon emissions while saving money, while the "top-down" macroeconomic models (and most optimization models) implicitly assume that no such options are possible since in the equilibrium situation they presume, these investments should already have occurred.
} 
Public Service Commission of Nevada has recently adopted a new Rule governing the utility planning process in that state, that also embodies these values (Nevada PSC, 1990). Somewhat higher values for $\mathrm{CO}_{2}-\$ 40$ per ton - have been embodied in the design of utility emissions taxes in Sweden, and have been proposed for resource planning decisions in Oregon.

Table 2.4

State Carbon Dioxide Valuations

\begin{tabular}{cl} 
State & $\begin{array}{l}\mathrm{CO}_{2} \text { Value } \\
\$ / \text { Ton }\end{array}$ \\
CA [1] & $\$ 7.50$ \\
NY [2] & $\$ 74$ \\
NY [3] & $\$ 5.50$ \\
NV [4] & $\$ 22$ \\
MA [5] & $\$ 23$ \\
OR [6] & $\$ 10-40$ \\
\hline
\end{tabular}

Notes:

[1] To be applied io 1991 resource plans (Utility Environment Report, 1991).

[2] Proposed values based on a genaral revenue tax in real levelized 1990 dollars (Draft New York State Energy Plan 1991 Biennial Update, 1991). Currently the

13) PSC requires that $\mathrm{CO}_{2}$ be valued at about $\$ 1$ per ton for new resource bidding

[3] Proposed values based on a trust fund tax in real levelized 1990 dollars

[4] 1990 real levelized dollars, Docket $89-752$

[5] Values adopted in Massachusetts Docket 89-239, converted to real levelized 1990 dollars.

[6] Proposed values submitted by Oregon Public Utility Commission/Department of Energy Staff, October 1991 (UM, 424).

Costs for the other greenhouse gases $\left(\mathrm{CH}_{4}\right.$ and $\left.\mathrm{N}_{2} \mathrm{O}\right)$ are based on the $\mathrm{CO}_{2}$ figure, assuming that they contribute, respectively, 10 and 180 times as much global warming, per unit weight, as does $\mathrm{CO}_{2}$ (Lashof and Ahuja, 1990). Thus, the $\mathrm{CH}_{4}$ cost is 10 times the $\mathrm{CO}_{2}$ cost and the $\mathrm{N}_{2} \mathrm{O}$ cost is 180 times the $\mathrm{CO}_{2}$ cost. 


\subsection{The Biomass Carbon Cycle}

Carbon is fixed in biomass through photosynthesis in which carbon dioxide is taken up from the atmosphere by the growing plant or tree. The carbon content of trees for example, is about one quarter their green weight or one half their dry weight. If a forest is in equilibrium for its hiomass production, there will be a balance between carbon uptake in growth and carbon releases from the aerobic decay of dead woody biomass (decomposition in the presence of oxygen). Essentially no net uptake or release of carbon dioxide takes place under such circumstances, but for small amounts of carbon that may become a part of the underlying soil, and small amounts of methane release from anaerobic decay. If an existing forest is still undergoing net growth (as is the case to some extent in New England) the uptake of carbon associated with the increases in overall biomass will exceed the decay of biomass, and a net sink for carbon will exist during the growth period.

The combustion of biomass for energy production releases carbon, primarily as carbon dioxide, just as the combustion of fossil fuels. At the same time, essentially the same amount of carbon will be taken from the atmosphere in the growth of the biomass. Thus, energy would be provided with essentially no net emissions of carbon dioxide if the biomass is used at the same rate that it is grown. If energy from such biomass resources were used instead of fossil fuels overall reductions of carbon dioxide releases would be effected. The use as fuels of forest and milling residues that would otherwise decompose can also provide energy at no net emissions of carbon dioxide, when employed as an alternative to fossil fuels.

\section{Wood Energy}

The energy and carbon content of woody biomass from forests can be compared for typical forests in the Northeastern U.S. and from potential high yield short rotation forest energy crops. One acre of forested land in the Northeastern U.S., with stocks of about 80 green tons per acre, is producing about 2 green tons per acre per year net growth. This would take up almost one-half ton of carbon (1.8 tons carbon dioxide) annually, and provide about $14 \mathrm{MMBtu}$ of fuel for combustion in furnaces or steamelectric power plants. At the high end it has been estimated that certain short rotation high yield species can provide 20 green tons per acre net annual yield, thereby providing about ten times the energy and carbon uptake per acre as the existing regional forests.

Below in Table 2.5 we compare the carbon dioxide emissions characteristics of various fossil electric generation options to biomass used in conventional wood steamelectric (boiler plus steam turbine) and advanced biomass gasification steam injected combustion turbine (BIG STIG) technologies. The fossil technologies include new integrated gasification combined cycle (IGCC) coal and new combined cycle natural gas (CCNG), since these are among the baseload facilities that are currently being considered throughout the U.S., and existing oil steam electric facilities, since these are 
likely candidates to be displaced by more economical options with favorable greenhouse gas characteristics.

Note that for the biomass facilities - BIG STIG and conventional WOOD steamelectric power plants - the gross emissions at the plant is given, as well as net emissions of zero which takes account of the growth of biomass to provide the fuel resource on a sustainable basis.

Table 2.5

Carbon Trade-otfs

Electric Sector

\begin{tabular}{|c|c|c|c|c|c|c|c|}
\hline & IGCC Coal & CCNG & Exist. Oil & \multicolumn{2}{|c|}{ BIG/STIG } & \multicolumn{2}{|c|}{ Wood } \\
\hline $\begin{array}{l}\text { Heat Rate } \\
\text { (MMBtu/MWh) }\end{array}$ & 9.2 & 7.7 & 10.0 & 10.5 & & 16. & \\
\hline $\begin{array}{l}\mathrm{CO}_{2} \text { Emiss } \\
\text { (lb/MMBtu) }\end{array}$ & 198 & 119 & 170 & $\begin{array}{r}213 \\
0\end{array}$ & $\begin{array}{c}\text { (Gross) } \\
\text { (Net) }\end{array}$ & $\begin{array}{r}213 \\
0\end{array}$ & $\begin{array}{l}\text { (Gross) } \\
\text { (Net) }\end{array}$ \\
\hline $\begin{array}{l}\mathrm{CO}_{2} \text { Emiss } \\
(\mathrm{lb} / \mathrm{MWh})\end{array}$ & 1820 & 920 & 1700 & $\begin{array}{r}2222 \\
0\end{array}$ & $\begin{array}{c}\text { (Gross) } \\
\text { (Net) }\end{array}$ & $\begin{array}{r}3520 \\
0\end{array}$ & $\begin{array}{l}\text { (Gross) } \\
\text { (Net) }\end{array}$ \\
\hline $\begin{array}{l}\text { C Emissions }{ }^{10} \\
\text { (lb/MWh) }\end{array}$ & 500 & 260 & 460 & $\begin{array}{r}600 \\
0\end{array}$ & $\begin{array}{l}\text { (Gross) } \\
\text { (Net) }\end{array}$ & $\begin{array}{r}920 \\
0\end{array}$ & $\begin{array}{l}\text { (Gross) } \\
\text { (Net) }\end{array}$ \\
\hline
\end{tabular}

While new coal plants will have energy efficiency improvements of more than 10 percent relative to existing coal plants, and sulphur and nitrogen dioxide emissions much lower than current levels owing to pollution control equipment, carbon dioxide will be essentially unchanged at between 0.91 to 1.0 tons per MWh generated. The principal supply side alternative to coal is natural gas, which in a combined cycle facility releases about one-half the carbon as a coal plant. Advanced fuel cells, which may become commercial and economical over the next twenty years, may provide additional reductions owing to their greater efficiency (with heat rates approaching 6 $\mathrm{MMBtu} / \mathrm{MWh}$ ). On the other hand, renewable energy resources and their technologies - solar, wind, geothermal, and hydro, and biomass in particular - can provide energy with no net releases of carbon dioxide.

Thus, $1000 \mathrm{MW}$ of BIG STIG at a 75 percent capacity factor will save about 0.85 to 1.54 Million Tons carbon (C) annually if it displaces fossil fuel power plants and if the biomass is supplied in a sustainable manner. The same amount of generation from a conventional wood-electric power plant would provide the same displacement,

${ }^{10} \mathrm{CO}_{2}$ emissions can be expressed as carbon (C) emissions, by stating the carbon content by weight in $\mathrm{CO}_{2}$, which is $12 / 44$ times the weight of $\mathrm{CO}_{2}$ 
but would require about 50 percent more wood resources (and thereby land) and would tend to have significantly more emissions of other air pollutants from combustion."

\section{Municipal Solid Waste}

Solid waste is an important source of greenhouse gases: primarily $\mathrm{CO}_{2}$ from combustion, and $\mathrm{CH}_{4}$ from decay of carbonaceous matter in landfill. Since the organic carbon is taken up from the atmosphere in photosynthesis, there is potentially a net carbon balance, if the biomass (e.g., paper and agricultural products) is produced in a sustainable cycle. There are two factors, however, that make the solid waste biomass cycle particularly important: first, given the higher global warming potential of methane, the carbon that decays in landfill will contribute more to global warming than the same amount taken up in biomass growth; second, whatever the mode of decay, solid waste can be used to replace fossil fuels, and thus have carbon uptake to balance their carbon releases.

Municipal solid waste disposed of in landfills will release equal amounts of carbon through its decomposition as carbon dioxide and methane (thus $\mathrm{CO}_{2}$ and $\mathrm{CH}_{4}$ in a 3 to 1 ratio by weight). Alternatively, the landfill gases could be captured and burned for heat and/or electricity, the solid waste could be incinerated to produce heat and/or electricity, or the organic fraction of the waste could be made into compost. In these instances almost all of the carbon is released as carbon dioxide which, from a global warming perspective, is preferable to methane emissions owing to the higher global warming potential of methane per unit of weight. Thus, while the biomass in the solid waste (paper, woody products, other organic matter) may be produced sustainably in a closed carbon cycle, with carbon uptake equal to carbon releases, it makes a difference whether the carbon released is in carbon dioxide or in methane.

Assuming that about 23 percent of municipal solid waste is degradable carbon, that 80 percent is fully degraded, and that equal amounts of methane and carbon dioxide are released by volume (i.e., equal amounts of carbon), one million tons of solid waste would release: $:^{12}$

$\begin{array}{ll}\text { Methane } & 0.23 * 0.8 * 0.5 * 16 / 12=0.12 \text { Million Tons } \\ \text { Carbon Dioxide } & 0.23 * 0.8 * 0.5 * 44 / 12=0.34 \text { Million Tons }\end{array}$

\footnotetext{
"Of course, it is the relative efficiencies of the BIG STIG and conventional wood-electric technologies that gives rise to these differences. It is noteworthy, therefore, that a range of heat rates have been indicated for these technologies, 8.5 to $10.5 \mathrm{MMBtu} / \mathrm{MWh}$ for BIG STIGS, and 12 to $20 \mathrm{MMBtu} / \mathrm{MWH}$ for conventional wood-electric power plants. Thus, the BIG STIGS generally look much more favorable in efficiency, although the conventional wood-electric facilities might come close (with about $15 \%$ lower efficiency) under some circumstances.

${ }^{12}$ Note that New York state generates about 15 million tons of municipal solid waste per year.
} 
The methane would have an ene:gy content of about 5.7 MMBtu, while the original solid waste would have an energy content of about $9 \mathrm{MMBtu}$. In carbon dioxide or carbon global warming potential equivalents, using a methane GWP of 10.0 in $\mathrm{CO}_{2}$ equivalent), the releases would be:
Methane 1.20 Million Tons $\mathrm{CO}_{2}$ equiv Carbon Dioxide 0.34 Million Tons

Some examples of the trade-offs between different means of managing the solid waste stream are given below. These include: conventional landfill, combustion of captured landfill methane and displacing oil combustion, incinerating solid waste for electric generation and displacing coal-fired generation, and composting.

Case 1. Conventional Landfill (one million tons per year)

GWP (C equiv)

$$
\begin{aligned}
& \text { Annual Emissions (106 tons) } \\
& \qquad \mathrm{CO}_{2}
\end{aligned}
$$

Landfill

Releases ( $C$ equiv) ${ }^{13}$

Biomass Growth

Offset ( $C$ equiv)

Net (C equiv)
0.34

0.09

$(0.67)$

(0.18)

(0.09)
Methane

\begin{tabular}{|c|c|}
\hline 0.12 & -..- \\
\hline 0.33 & 0.42 \\
\hline$\ldots$ & $\cdots$ \\
\hline - & $(0.18)$ \\
\hline 0.33 & 0.24 \\
\hline
\end{tabular}

Total

Thus, despite overall carbon balances between growth and release, a net GWP of 0.24 million tons in carbon equivalent (or 0.88 in $\mathrm{CO}_{2}$ equivalent) is created. ${ }^{14}$

By comparison, another option is to capture the methane released and burn it to provide thermal energy, thereby releasing all of the carbon as carbon dioxide. At the same time, the energy can displace fossil fuel at the end-use. In this case there is a net reduction of GWP (in C equivalent) of 0.14 million tons.

\footnotetext{
${ }^{13}$ GWP (in C equivalent), using 7.09 for methane and $12 / 44=0.27$ for $\mathrm{CO}_{2}$ :

14 The landfill releases of carbon are $12 / 44 \times 0.31$ plus $12 / 16 \times 0.11=0.17$, based on the relative molecular weights of carbon (12), carbon dioxide (44), and methane (16).
} 
Case 2. Capture Methane and Displace Oil at End-Use

\begin{tabular}{|c|c|c|c|}
\hline & Annual & imissions ( $10^{6}$ tons) & \\
\hline & $\mathrm{CO}_{2}$ & Methane & Total GWP (in C equiv) \\
\hline Landfill Gas Combustion & 0.67 & $\ldots$ & $\cdots$ \\
\hline Releases ( $C$ equiv) & 0.18 & - & 0.18 \\
\hline Biomass Growth & $(0.67)$ & ...- & $\ldots$ \\
\hline Offset (C equiv) & $(0.18)$ & - & $(0.18)$ \\
\hline Oil Emissions & $(0.50)$ & $\cdots$ & $(0.50)$ \\
\hline Offset ( $C$ equiv) & $(0.14)$ & $=$ & $(0.14)$ \\
\hline Net ( $C$ equiv) & $(0.14)$ & - & $(0.14)$ \\
\hline
\end{tabular}

The example below examines incineration of solid waste and displacement of coal-fired electricity production. One million tons of MSW has an energy content of about $9 \mathrm{MMBtu}$. Assuming a heat rate of $16.5 \mathrm{MMBtu} / \mathrm{MWh}$, about $0.545 \mathrm{MWh}$ of electricity would be generated. The carbon dioxide emissions factor of MSW is 165 $\mathrm{lb} / \mathrm{MMBtu}(0.083$ tons/MMBtu) or 1.36 tons/MWh. We assume that this displaces coal-fired generation with a heat rate of $10 \mathrm{MMBtu} / \mathrm{MWh}$ and a carbon dioxide emissions factor of $210 \mathrm{lb} / \mathrm{MMBtu}(0.11 \mathrm{ton} / \mathrm{MMBtu})$ or 1.05 tons/MWh.

Case 3. Burn for Electricity and Displace Coal-fired Generation

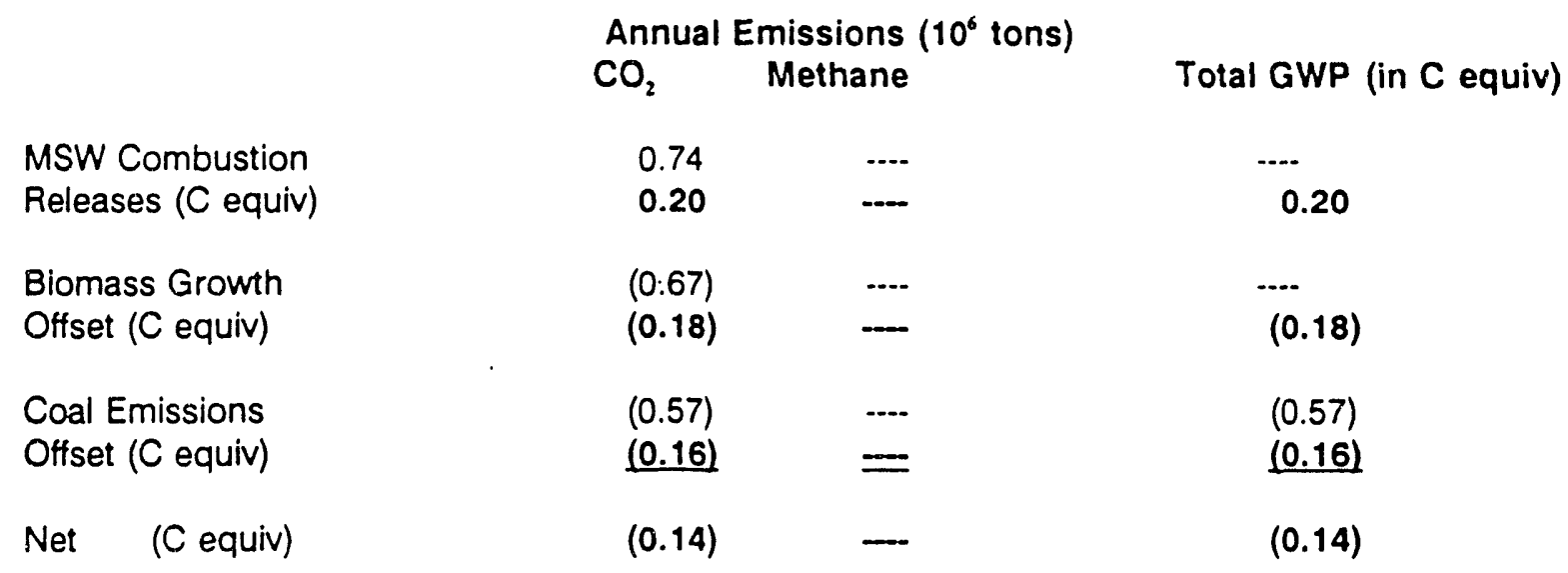


Case 4. Composting

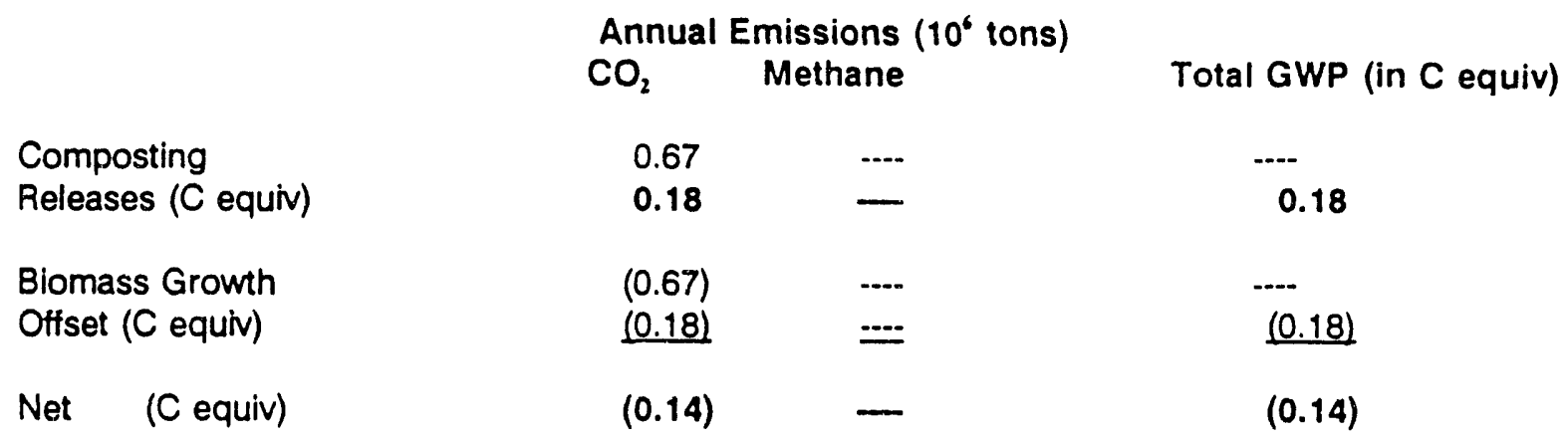

\subsection{Economic and Environmental Considerations in Biomass Energy Usage}

Significant levels of biomass energy usage could make use of forest and agricultural residues, thereby displacing fossil fuels combustion with combustion of carbonaceous matter that would have otherwise released carbon dioxide as it decayed. Such usage, however, might be limited by the ecological requirements met by closing the carbon cycle locally. The harvesting and movement of biomass from forests may also introduce specific environmental impacts, some deleterious, and perhaps some advantageous through introducing better management systems to already working forests. Large scale biomass energy forestry and agriculture can also require large amounts of land and water resources, and they could potentially disrupt sensitive ecological systems unless careful protocols are followed.

Municipal solid waste and sewage can provide energy feedstocks through incineration and or the capture and use of methane to produce thermal and/or electric energy. These options interact very strongly with other societal objectives, particularly as population and consumption increase the production of waste and as local air and water pollution and land-use become increasingly problematical. Solid waste used for energy production will compete with and to some degree complement source reduction, recycling and composting.

The principal environmental benefit of using biofuels is the inherent balance between carbon capture from the atmosphere in photosynthesis and carbon release at combustion and refining. Atmospheric carbon will essentially be neutral for a mature self-sustainable biofuel cycle. This does not necessarily imply that the transition to a biofuel cycle will not affect atmospheric carbon balances.

The net gain or loss of atmospheric carbon associated with the use of biofuels is a function of three factors - alternative fuels/efficiency, biomass harvesting/collecting 
and conversion practices, and alternative land uses. The first factor, alternatives to biofuels, is currently considered the overwhelming factor. In the near-term, biofuels may to some degree displace fossil fuels, which are major contributors to atmospheric carbon. Over time, however, competition with other technically feasible alternatives to biofuels (e.g., solar photovoltaic produced hydrogen) may decrease the importance of this factor relative to the others.

Biomass harvesting and collecting practices may place an additional penalty on the carbon balance as they liberate carbon captured in disrupted soils. Fertilization may create a further penalty depending on the fertilizer feedstock. The greatest penalties in these areas are likely to occur at the time that land is converted to dedicated biomass production. The conversion of biomass to more i efined biofuels may also have an impact on greenhouse gases, especially if converted to methane which has a high $\mathrm{CO}_{2}$ equivalence and which can leak during production and distribution. Furthermore, large scale energy production and harvesting could create significant disruptions to a far larger part of the ecosystem than the area under cultivation itself, ultimately creating negative implications for carbon capture and retention.

Alternative land uses may have a significant impact on the contribution of biofuels to the carbon balance versus alternative energy sources. A biofuel cycle based upon dedicated biomass production is expected to be in rough balance between captured and released carbon. However, alternative uses of the land - e.g., afforestation - may provide pure carbon sinks, while the biomass is undergoing growth. 


\section{CONEG Region Energy and Solid Waste Systems}

In this section, we develop projections of energy demand by sector and fuel type for the eleven CONEG states, including demand for biomass fuel resources (firewood, MSW, landfill gas), for the next thirty years. In addition, we project the demand for wood products (paper and pulp, construction), as these will ultimately affect the carbon cycle in the region. From the end-use fuel demands, we estimate the carbon dioxide and methane emissions from combustion - both at the point of end-use and for electricity production to supply the regional electric power grids - and from extraction and distribution losses.

We provide both a Reference Case and an Energy Conservation Case projection for purposes of this study. The purpose of the Reference projection is not to provide a prediction of the future energy system in the region. Rather, it is provided as a plausible reference path, based upon continuation of current trends and policies, from which an assessment of greenhouse gas emissions and the potential contribution of biomass to the mitigation of these emissions can be made.

The Conservation Case is provided in order to provide a context for biomass related greenhouse gas mitigation strategies. Given the very ambitious reduction goals that may be needed for climate stdbilization, biomass will likely play a modest role within a mix of many energy supply and demand options. Energy conservation, which is itself a set of myriad technologies and practices across all end-uses, can provide significant levels of carbon dioxide reductions at little or no net cost.

\subsection{The Current Energy System}

The CONEG region has a diverse energy system with a variety of resources, including a small but significant biomass contribution. The region has about 24 percent of the nation's population, and consumes about 17 percent of national primary energy requirements. The share of oil and nuclear energy in the regional total are above their share of the national total, about 50 and 10 percent, respectively, compared with 40 and 7 percent. On the other hand, its coal and natural gas contributions are lower than the nation's, at about 16 and 18 percent, respectively, versus 23 and 22 percent.

Almost all of the coal consumption in the region occurs in four states - New York, New Jersey, Pennsylvania, and Maryland. As noted earlier, the combustion of coal contributes about twice as much carbon to the atmosphere as natural gas and about twenty-five percent more than oil. From the standpoint of overall carbon emissions, then, the region's relatively lower coal and natural gas shares tend to off-set one another. 
The current end-use energy demands for the CONEG region are given on Table 3.1 below, organized by three sub-regions: New England, Mid-Atlantic, and New York. (Note that commercial wood is included here as a part of the renewable resources within total end-use energy demand.)

Table 3.1

CONEG Region

1988 End-Use Energy Demands

Quadrillion Biu

\begin{tabular}{|c|c|c|c|c|c|}
\hline \multirow[b]{2}{*}{$\begin{array}{l}\text { Coal } \\
\text { Nat Gas } \\
\text { Oil } \\
\text { Renew } \\
\text { Electric }\end{array}$} & & $\begin{array}{l}\text { Mid-All } \\
0.50 \\
1.26\end{array}$ & $\begin{array}{l}\text { New York } \\
0.10 \\
0.66\end{array}$ & \multicolumn{2}{|c|}{ CONEG } \\
\hline & $\begin{array}{l}0.02 \\
0.31 \\
1.52 \\
0.27 \\
0.35 \\
\end{array}$ & $\begin{array}{l}0.50 \\
1.26 \\
2.89 \\
0.17 \\
\underline{0.79}\end{array}$ & $\begin{array}{l}0.10 \\
0.66 \\
1.38 \\
0.12 \\
\underline{0.43} \\
\end{array}$ & $\begin{array}{l}0.62 \\
2.23 \\
5.79 \\
0.56 \\
1.57 \\
\end{array}$ & $\begin{array}{r}(5.8 \%) \\
(20.7 \%) \\
(53.8 \%) \\
(5.2 \%) \\
(14.6 \%)\end{array}$ \\
\hline Total & 2.47 & 5.60 & 2.69 & 10.76 & $(100.0 \%)$ \\
\hline
\end{tabular}

NB: Renewables include 0.12, 0.05, and 0.4 Quadrillion Btu, for New England, Mid-Atlartic and New
York, respectively.

Owing to the conversion efficiency of electricity production - about 34 percent the end-use electricity demands have primary energy requirements about three times their value at the end-use in the table above, or about 4.58 Quads for all of the CONEG region (1.03 Quads for New England, 2.3 Quads for Mid-Atlantic, and 1.25
Quads for New York).

The breakdown of end-use renewable resources is given in Table 3.2 below. The contribution of end-use firewood to overall energy end-use demand is about 3 percent for the CONEG region and as high as about 5 percent for New England. The contribution of all wood uses (including commercial wood) is about 5 percent for the CONEG region as a whole, and as high as 10 percent for New England. For all enduse renewables it is about 5 percent and 11 percent for the CONEG states and New England, respectively. 


\begin{tabular}{lrrrr} 
& Now Eng & Mid-Atl & New York & CONEG \\
Firewood & 126 & 112 & 76 & 314 \\
Wood Prod & 46 & 30 & 18 & 94 \\
Paper \& Pulp & 79 & 22 & 17 & 118 \\
Landf Gas & 1 & 2 & 2 & 5 \\
MSW Steam & 7 & 8 & 5 & 20 \\
Hydro & 13 & $=$ & 2 & 15 \\
Total & 272 & 174 & 120 & 566 \\
\hline
\end{tabular}

The breakdown of electric energy generation into the principal fuels consumed is given in Table 3.3 below. Note that while wood contributes less than 1 percent on a CONEG regional basis, it contributes almost 4 percent in New England. Similarly, biomass contributes about 6 percent to total electricity in New England, but only 2 percent for the CONEG region as a whole.

Table 3.3

CONEG, 1988 Electric Generation Mix Million MWhrs

\begin{tabular}{lrrrr}
\hline & New Eng & Mid-Atl & New York & CONEG \\
Coal & & & & \\
Nat Gas & 15.0 & 132.8 & 26.6 & 174.4 \\
Oil & 1.9 & 4.9 & 14.0 & 20.8 \\
Nucl & 38.2 & 18.7 & 39.7 & 96.6 \\
Hydro & 32.8 & 66.3 & 23.6 & 122.7 \\
Fossil Imports & 6.1 & 6.5 & 25.8 & 38.5 \\
Hydro Imports & 3.1 & 23.3 & 2.0 & 28.4 \\
Wood & 9.4 &.-- & 5.9 & 15.3 \\
MSW & 4.1 & 0.4 & 0.1 & 4.6 \\
Landfill Gas & 2.7 & 0.6 & 1.3 & 4.6 \\
Other & 0.2 & 0.3 & 0.2 & 0.7 \\
& -- & 1.5 & 0.1 & 1.6 \\
Total & & & & 508.4 \\
& 113.6 & 255.4 & 139.4 & $0.9 \%$ \\
Wood Share & & & & $2.0 \%$ \\
Biomass Share & $3.6 \%$ & $0.2 \%$ & $0.1 \%$ & $12.5 \%$ \\
Renewable Share & $6.2 \%$ & $0.5 \%$ & $1.2 \%$ & \\
\hline
\end{tabular}


Note that the 4.6 million $\mathrm{MWh}$ contribution from wood-electricity, shown in Table 3.3, amounts to about 75 Trillion Btu, which is about 24 percent of the firewood used in the buildings and industrial sectors and about 14 percent of the total end-use wood demand (firewood and wood/paper products) in the region. MSW-electricity also contributes about 75 trillion Btu, but this is almost four times MSW use for building energy and 75 percent of total MSW energy use in the region.

A more detailed breakdown of the contributions of biomass to electric generating capacity in the CONEG states, broken out by wood combustion, municipal solid waste (MSW) combustion, and landfill gas combustion, is given below in Table 3.4. For consistency in these biomass energy accounts we used the results of the Public Citizen survey of many sources and agencies in The Power of the States (Rader, 1990).

Table 3.4

CONEG Reference Case

1988 Biomass-Electric Power (MW)

\begin{tabular}{|c|c|c|c|c|}
\hline & Wood & MSW & Landfill & $\begin{array}{c}\text { Biomass } \\
\text { Total }\end{array}$ \\
\hline Connecticut & 0.3 & 152.2 & 2.4 & 154.9 \\
\hline Maine & 543.0 & 83.0 & $\cdots$ & 626.0 \\
\hline Massachusetts & 0.9 & 211.0 & 14.4 & 226.3 \\
\hline New Hampshire & 117.0 & 18.0 & 1.0 & 136.0 \\
\hline Rhode Island & $\cdots$ & $-\cdots$ & 8.0 & 8.0 \\
\hline Vermont & 54.7 & 5.5 & 0.1 & 60.3 \\
\hline New England & 715.9 & 469.7 & 25.9 & 1211.5 \\
\hline Delaware & -.--- & 19.0 & $-\cdots$ & 19.0 \\
\hline Maryland & 5.0 & 57.0 & 5.5 & 67.5 \\
\hline New Jersey & $\ldots$ & 12.0 & 8.0 & 20.0 \\
\hline Pennsylvania & 72.3 & 16.3 & 17.5 & 106.1 \\
\hline Mid-Atlantic & 77.3 & 104.3 & 31.0 & 212.6 \\
\hline New York & 13.0 & 191.0 & 31.0 & 235.0 \\
\hline CONEG & 806.2 & 765.0 & 87.9 & 1659.1 \\
\hline
\end{tabular}




\subsection{Reference Case Energy Projections}

\section{End-Use Energy Demands}

Projections of end-use energy requirements for various fuel types (including biomass fuels) and for non-fuel wood products for the 1990 to 2020 period were established for four sectors - residential, commercial, industrial, and transportation - in each of the eleven CONEG states. The end-use resources accounted for were:

$\begin{array}{llll}\text { Fossil Fuel } & \text { Biomass Energy } & \text { Biomass Products } & \text { Electricity } \\ \text { Natural Gas } & \text { Resid Firewood } & \begin{array}{l}\text { Pulp and Paper } \\ \text { Gas }\end{array} & \\ \text { Gasoline } & \text { Comm/Ind Woodfuel }{ }^{15} \text { Construction Wood } & \\ \text { Aviation Gas } & \text { Landfill Gas } & \\ \text { Kerosene/Jet Fuel } & \text { Stearn from MSW } & \\ \text { Diesel/Gas Oil } & & \\ \text { Residual/Fuel Oil } & & \\ \text { LPG/Bottled Gas } & & \\ \text { Other Petro Products } & & \\ \text { Coal } & \end{array}$

In the list above we include all energy resources that directly serve end-uses, whether or not the combustion (and emissions) is at the point of end-use. For both electricity and steam from MSW the combustion takes place at an earlier stage. As shown earlier for the year 1988, electricity supplied to meet end-use requirements is generated by a variety of fuels, including coal, oil, natural gas, nuclear, etc. as well as wood, landfill gas, and MSW combusted for electric generation.

The Base Year demands (1988) for conventional end-use fuels were taken from the Department of Energy EIA State Energy Data System (SEDS, 1988), as this was the most recent single comprehensive source available at the state/sectoral level. ${ }^{17}$

\footnotetext{
${ }^{15}$ Both firewood and pulp and paper are short lived wood products that through combustion or decay contribute $\mathrm{CO}_{2}$ or $\mathrm{CH}_{4}$ to the atmosphere. Pulp and paper decay is accounted for as part of overall landfill emissions which could be reduced by recycling.

${ }^{16}$ We have tracked construction wood using zero emissions coefficients for greenhouse gases as the carbon remains sequestered over the time-frame of our analysis.

17 The SEDS state/sector energy end-use data for 1988 were compared to a variety of other sources, including the New York State Energy Office (SEO, 1989), the New England Power Pool (NEPOOL, 1988) and the U.S. Department of Energy Annual Energy Outlook (DOE/EIA, 1990). Aggregate electricity
} 
The state end-use demands for biomass energy were more difficult to estimate. Several sources - including the EPA's National Emissions Data System (NEDS, 1988), Public Citizen's The Power of the States (PC, 1990), the New England Power Pool (NEPOOL, 1988)), the Governmental Advisory Associates Methane Recovery From Landfill Yearbook (GAA, 1989a), the New York State Energy Office (SEO, 1989b), the U.S. Forest Service (USFS, 1988), the National Wood Energy Association (NWEA, 1988) and reports by Tellus Institute (Tellus, 1987) - were consulted, providing a range of estimates, using a variety of assumptions, for all or some of the biomass resource demands. ${ }^{18}$ Reasonable "best estimates" were made on the basis of this survey, largely conforming to the Public Citizen estimates. ${ }^{19}$ State-specific paper and pulp, and construction wood demands were obtained from the U.S. Forest Service (USFS, 1988).

Reference scenario end-use energy demand projections for conventional fuels were based upon the state-level forecasts embodied in the U.S. Department of Energy's EIA 1990 Annual Energy Outlook (DOE/EIA, 1990), as these were the most recent comprehensive energy forecasts, for as long as twenty years, covering all the CONEG states using a single consistent methodology. However, comparison of these electricity forecasts with those provided by the utilities in the region - in (NERC, 1989), (NEPOOL, 1988), (SEO, 1989) - and by the Tellus Institute, indicated that a considerably lower level of electricity load growth would be appropriate. Consequently, for simplicity, a uniform scale-back of the annual rates of load growth in (DOE/ELA, 1990) by two-thirds was adopted for the residential, commercial and industrial sectors in all the states. ${ }^{20}$ The overall energy demands in each sector were kept the same, so that the reduced electricity demands were compensated by increases in the fossil fuel demands apportioned pro rata across the various fossil fuels according to the magnitude of their demands.

The energy end-use growth rates assumed for this study are listed in Table. 3.5.

requirements from the states forming power pools (NYPP, NEPOOL, MAAC/PJM) were compared to data from the North American Electric Reliability Council (NERC, 1989). The results were comparable.

${ }^{18}$ Only the U.S. Forest Service (USFS, 1988) gave pulp and construction wood demands. Only Public Citizen gave wood-electric capacities as well as direct wood use in the residential and commercial sectors.

19 The Public Citizen report (PC, 1990) on state renewable energy activity, itself reviewed a large number of sources. For firewood the Public Citizen report used the NWEA estimates.

${ }^{20}$ The DOE/EIA projections were for the 1988 to 2010 period. For the 2010 to 2020 period the load growth over the 2000 to 2010 period (lower than that for the 1988 to 2000 period) was assumed to continue, appropriately scaled by two-thirds. 
Table 3.5

CONEG Region, Reference Scenario

End-Use Demund Growth Rates

Percent/Year

\begin{tabular}{cccc}
\hline & $1988-2004$ & $2004-2020$ & $1988-2020$ \\
New England & & & \\
Electricity & 1.85 & 1.52 & 1.68 \\
All Energy & 0.40 & 0.54 & 0.47 \\
Mid-Attantic & & & 1.60 \\
Electricity & 1.74 & 1.47 & 0.74 \\
All Energy & 0.65 & 0.81 & \\
New York & & & 1.54 \\
Electricity & 1.65 & 1.41 & 0.42 \\
All Energy & 0.35 & 0.50 & \\
\hline
\end{tabular}

Sectoral fuelwood demands were assumed to grow at the same rate as overall energy demand for each sector. Pulp wood and construction wood were projected based on the Department of Commerce $U$. S. Industrial Outlook; 1.33 percent/year for paper and pulp (except for Maine, which was projected tn grow at 1.19 percent/year), and 1.50 percent/year for construction wood products (except for Maine, which was projected to decline at 3.67 percent/year.) Municipal Solid Waste facilities providing end-use energy (MSW steam and landfill gas) were assumed to remain constant at their current levels throughout the forecast period.

\section{End-Use Demand Projections}

In Tables 3.6a, 3.6b and 3.6c, the aggregate end-use fuel consumption projections used for the purpose of this study are given for New England, the Mid-Atlantic and New York, respectively. The fuel use breakdown is essentially that of the DOE/EIA 1990 Annual Energy Outlook, with the modifications discussed earlier. 
Table $3.6 a$

Reference Case

End-Use Energy Projections

New England

Trillion Btu

\begin{tabular}{lrrrrr}
\hline & 1988 & 1996 & 2004 & 2012 & 2020 \\
& & & & & \\
Electricity & 349.2 & 408.6 & 467.9 & 529.0 & 595.0 \\
Natural Gas & 311.7 & 332.1 & 352.5 & 370.4 & 380.5 \\
Oil & 1521.4 & 1513.3 & 1505.9 & 1509.3 & 1548.0 \\
Coal & 17.8 & 19.2 & 20.8 & 22.9 & 27.2 \\
Renewable & 271.8 & 279.8 & 287.2 & 298.8 & 319.9 \\
Total New Eng & 2471.9 & 2553.0 & 2634.3 & 2731.0 & 2870.6 \\
& & & & & \\
Connecticut & 553.9 & 577.3 & 600.8 & 627.7 & 664.9 \\
Maine & 401.6 & 407.5 & 413.5 & 424.9 & 452.7 \\
Massachusetts & 1020.6 & 1049.3 & 1078.0 & 1108.8 & 1145.7 \\
New Hamphire & 211.7 & 224.5 & 237.4 & 252.0 & 271.9 \\
Rhode Island & 160.3 & 163.1 & 165.8 & 170.0 & 178.3 \\
Vermont & 124.0 & 131.4 & 138.8 & 147.0 & 157.1 \\
\hline
\end{tabular}

Table 3.6b

Reference Case

End-Use Energy Projections

Mid-Atlantic

Trillion Btu

\begin{tabular}{lrrrrr}
\hline & 1988 & 1996 & 2004 & 2012 & 2020 \\
Electricity & 785.1 & 909.7 & 1034.4 & 1163.5 & 1306.3 \\
Natural Gas & 1262.1 & 1314.2 & 1366.3 & 1421.8 & 1487.7 \\
Oil & 2888.3 & 2952.1 & 3016.0 & 3100.8 & 3248.9 \\
Coal & 495.4 & 552.2 & 608.9 & 686.3 & 825.2 \\
Renewable & 174.0 & 183.7 & 193.8 & 204.6 & 217.6 \\
& & & & & 7085.7 \\
Total Mid-Atl & 5604.9 & 5911.9 & 6219.4 & 6577.1 & 232.1 \\
Delaware & 175.3 & 187.0 & 198.7 & 212.4 & 1190.5 \\
Maryland & 869.8 & 934.0 & 998.0 & 1075.0 & 2090.6 \\
New Jersey & 1781.1 & 1843.2 & 1905.3 & 1979.6 & 3572.6 \\
Pennsylvania & 2778.5 & 2947.7 & 3117.3 & 3310.2 & \\
& & & & & \\
\hline
\end{tabular}


Table 3.6c

Reference Case

End-Use Energy Projections

New York

Trillion Btu

\begin{tabular}{lrrrrr}
\hline & 1988 & 1996 & 2004 & 2012 & 2020 \\
Electricity & 428.7 & 493.5 & 558.2 & 625.3 & 699.2 \\
Natural Gas & 660.1 & 691.6 & 723.1 & 751.9 & 772.9 \\
Oil & 1381.9 & 1345.1 & 1308.2 & 1283.7 & 1295.9 \\
Coal & 99.2 & 110.2 & 121.2 & 136.5 & 164.6 \\
Renewable & 120.4 & 126.6 & 133.0 & 139.3 & 145.7 \\
Total New York & 2690.3 & 2766.9 & 2843.7 & 2936.7 & 3078.3 \\
\hline
\end{tabular}

\section{Energy Supply}

\section{Electric Power System}

Power plants and their characteristics were specified for each region (New York, New England and Mid-Atlantic) based on data obtained from the North American Electric Reliability Council's 1989 Electricity Supply and Demand (NERC, 1989) and from state sources. ${ }^{21}$ Each region's electricity system is reflected in the three regional power pools - NEPOOL PJM (or MAAC) and NYPP. Existing facilities and those planned for the next ten years were specified explicitly by fuel type:

Coal Steam

Natural Gas Steam

Residual Steam

Natural Gas Combined Cycle

Distillate Combined Cycle

Nuclear

Hydro

Natural Gas Combustion Turbine
Distillate Combustion Turbine

Pumped Storage Hydro

Imports-Fossil

Imports-Hydro

MSW-Electric

Wood-Electric

Landfill Gas-Electric

Other Non Utility Generators 22

${ }^{21}$ The Mid-Atlantic Region encompasses the PJM system plus a few utilities in western Pennsylvania. Thus, the NERC PJM data was supplemented by data on the additional utilities.

2 Other Non Utility Generators were assumed to be a mix of natural gas combined cycle (25\%), natural gas combined cycle with cogeneration (25\%), atmospheric fluidized bed coal with cogeneration (25\%), and nonpolluting renewables such as hydro, wind or solar $(25 \%)$. 
With growth in electricity demand, combined with retirement of existing power supplies, new resources are needed over time. If the systems needed additional resources, as electricity demands increased and existing plants are retired, they were chosen by the LEAP model from IGCC coil and Natural Gas Combined Cycle for baseload and Natural Gas Combustion Turbines for peaking.

The contributions from MSW-electric facilities were assumed to increase over the next decade based upon recent planned additions. Landfill gas-electric facilities were assumed to remain constant over time. The role of wood-electric power was assumed to approximately double over the next two decades, with capacity additions at about one-third those embodied in the recent U.S. National Energy Strategy Reference Case. The projections for wood, MSW, and landfill gas-electric power are given below in Table 3.7.

Table 3.7

Reference Case

Biomass Electric Capacity

\begin{tabular}{|c|c|c|c|c|c|}
\hline & 1988 & 1996 & 2004 & 2012 & 2020 \\
\hline \multicolumn{6}{|l|}{ New England } \\
\hline Wood ST & 716 & 739 & 763 & 786 & 786 \\
\hline MSW ST & 470 & 520 & 570 & 570 & 570 \\
\hline Landf ST & $\underline{26}$ & $\underline{26}$ & 26 & $\underline{26}$ & $\underline{26}$ \\
\hline BIOMASS TOTAL & 1,212 & $1,2 \overline{285}$ & 1,359 & $1,3 \overline{82}$ & $1,3 \overline{82}$ \\
\hline \multicolumn{6}{|l|}{ TOTAL NEW ENG } \\
\hline CAPACITY & 22,947 & 27,024 & 31,779 & 36,660 & 41,237 \\
\hline \multicolumn{6}{|l|}{ Mid-Atlantic } \\
\hline Wood ST & 77 & 210 & 344 & 477 & 477 \\
\hline MSW ST & 104 & 324 & 544 & 544 & 544 \\
\hline Landf ST & $\frac{31}{210}$ & 31 & $\frac{31}{010}$ & $\underline{31}$ & $\frac{31}{50}$ \\
\hline BIOMASS TOTAL & 212 & 565 & 919 & 1,052 & 1,052 \\
\hline \multicolumn{6}{|l|}{ TOTAL MID-ATL } \\
\hline CAPACITY & 55,581 & 67,587 & 74,277 & 81,510 & 91,506 \\
\hline \multicolumn{6}{|l|}{ New York } \\
\hline Wood ST & 13 & 146 & 280 & 413 & 413 \\
\hline MSW ST & 191 & 224 & 257 & 257 & 257 \\
\hline Landf ST & 31 & 31 & 31 & 31 & 31 \\
\hline BIOMASS TOTAL & 235 & 401 & $5 \overline{68}$ & $7 \overline{701}$ & 701 \\
\hline $\begin{array}{l}\text { TOTAL NY } \\
\text { CAPACITY }\end{array}$ & 33.398 & 36.751 & 38.411 & 42630 & 47669 \\
\hline
\end{tabular}


While both wood-electric and MSW-electric capacity is assumed to about double over the study period, this roughly keeps pace with electric capacity growth as a whole. Thus, the biomass-electric share increases only modestly from about 1.5 percent to 1.8 percent of the total.

\section{Electricity Imports}

For each region net electricity imports were added to regional electric power production, to the degree that they are currently used and projected to meet electricity demand. This will have the effect of adding uses of energy resources (particularly coal in the Mid-Atlantic region) and their emissions from outside the regions to the regional accounts. This is perhaps one reason that our estimate of $\mathrm{CO}_{2}$ emissions in the CONEG region in 1988 is higher than the total reported earlier from the EPA study.

\section{Energy Losses}

Distribution losses (e.g., gas pipeline leaks), conversion losses in combustion (efficiencies, heat rates), or collection (water, small branches and roots for trees) were taken into account. Landfill gas captured for energy use was assumed to be composed of methane and carbon dioxide in approximately equal proportions by volume, the methane burned and the carbon dioxide directly released to the atmosphere. Landfill gas not captured was assumed to be released directly. Finally, it was assumed that for each region the demand for energy would be the determinant of the primary resources extracted (coal mining, gas extraction, oil production) or processed (oil refining), whether or not these processes occurred in those regions. Thus a unit of residual oil demand in New York is assigned the refinery use and oil extraction that it requires.

\section{Electricity Supply Projections}

Tables 3.8, 3.9, and 3.10 provide the electricity supply projections for the three regions. Note that we have assumed that existing oil and gas fired generating facilities will slowly retire over the study period, while existing coal units are assumed to remain in service, through life extension for those that might otherwise retire. We have also assumed that New England and New York will install new NGCC units for baseload supply, while the Mid-Atlantic will install new coal facilities. 
Table 3.8

Reference Case

Electric Generation Projections

New England

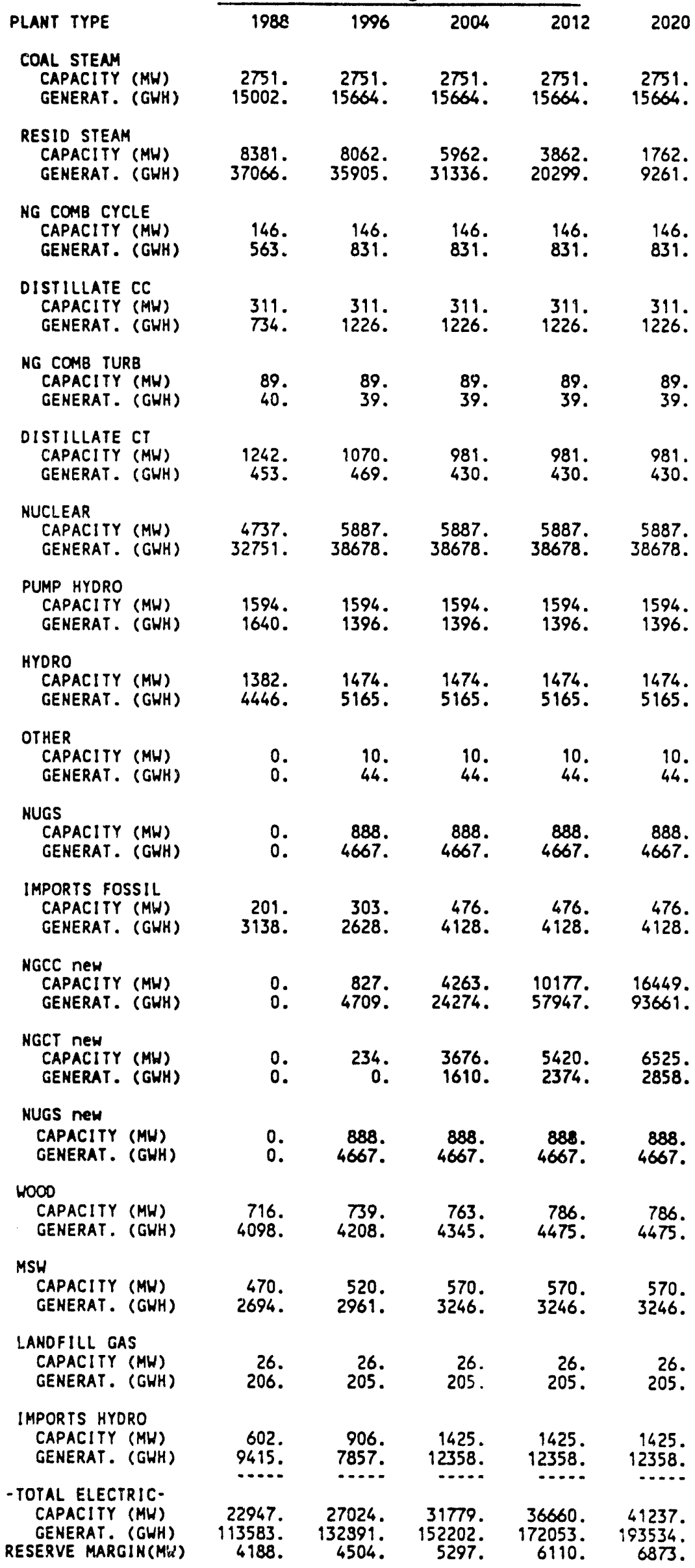


Table 3.9

Reference Case

Electric Generation Projections

Mid-Atlantic

\begin{tabular}{|c|c|c|c|c|c|}
\hline PLANT TYPE & 1988 & 1996 & 2004 & 2012 & 2020 \\
\hline $\begin{array}{l}\text { COAL STEAM } \\
\text { CAPACITY (MH) } \\
\text { GENERAT. (GHH) }\end{array}$ & $\begin{array}{r}22357 \\
132765\end{array}$ & $\begin{array}{l}22997 . \\
151090\end{array}$ & $\begin{array}{r}22997 \\
151090\end{array}$ & $\begin{array}{r}22997 . \\
151090\end{array}$ & $\begin{array}{r}22997 . \\
151090 .\end{array}$ \\
\hline $\begin{array}{l}\text { NG STEAM } \\
\text { CAPACITY (MW) } \\
\text { GENERAT. (GWH) }\end{array}$ & $\begin{array}{l}2121 . \\
3722 .\end{array}$ & $\begin{array}{l}2121 . \\
9290 .\end{array}$ & $\begin{array}{l}1621 . \\
7100 .\end{array}$ & $\begin{array}{l}1121 . \\
4910 .\end{array}$ & $\begin{array}{r}621 . \\
2720 .\end{array}$ \\
\hline $\begin{array}{l}\text { RESID STEAM } \\
\text { CAPACITY (MH) } \\
\text { GENERAT. (GHH) }\end{array}$ & $\begin{array}{l}7801 . \\
17112 .\end{array}$ & $\begin{array}{l}7801 . \\
6421 .\end{array}$ & $\begin{array}{r}5801 \\
25408\end{array}$ & $\begin{array}{l}3801 \\
16648\end{array}$ & $\begin{array}{l}1801 . \\
7888 .\end{array}$ \\
\hline $\begin{array}{l}\text { NG COMB CYCLE } \\
\text { CAPACITY (MW) } \\
\text { GENERAT. (GHH) }\end{array}$ & $\begin{array}{l}218 . \\
487 .\end{array}$ & $\begin{array}{r}218 . \\
1241\end{array}$ & $\begin{array}{r}218 . \\
1241 .\end{array}$ & $\begin{array}{l}218 . \\
1241 .\end{array}$ & $\begin{array}{r}218 . \\
124 \% .\end{array}$ \\
\hline $\begin{array}{l}\text { DISTILLATE CC } \\
\text { CAPACITY (MW) } \\
\text { GENERAT. (GWH) }\end{array}$ & $\begin{array}{l}114 . \\
405 .\end{array}$ & $\begin{array}{l}114 . \\
399 .\end{array}$ & $\begin{array}{l}114 . \\
399 .\end{array}$ & $\begin{array}{l}114 . \\
399 .\end{array}$ & $\begin{array}{l}114 . \\
399 .\end{array}$ \\
\hline $\begin{array}{l}\text { NG COMB TURB } \\
\text { CAPACITY (MW) } \\
\text { GENERAT. (GWH) }\end{array}$ & $\begin{array}{r}2645 . \\
767 .\end{array}$ & $\begin{array}{l}2645 . \\
1159 .\end{array}$ & $\begin{array}{l}2645 . \\
1159 .\end{array}$ & $\begin{array}{l}2645 . \\
1159 .\end{array}$ & $\begin{array}{l}2645 . \\
1159 .\end{array}$ \\
\hline $\begin{array}{l}\text { DISTILLATE CT } \\
\text { CAPACITY (MH) } \\
\text { GENERAT. (GWH) }\end{array}$ & $\begin{array}{l}4763 . \\
1227 .\end{array}$ & $\begin{array}{l}4763 . \\
2086 .\end{array}$ & $\begin{array}{l}4763 . \\
2086 .\end{array}$ & $\begin{array}{l}4763 . \\
2086 .\end{array}$ & $\begin{array}{l}4763 . \\
2086 .\end{array}$ \\
\hline $\begin{array}{l}\text { NUCLEAR } \\
\text { CAPACITY (MH) } \\
\text { GENERAT. (GWH) }\end{array}$ & $\begin{array}{l}12194 . \\
66320 .\end{array}$ & $\begin{array}{l}13249 \\
77761\end{array}$ & $\begin{array}{l}13249 \\
77761\end{array}$ & $\begin{array}{l}13249 . \\
77761 .\end{array}$ & $\begin{array}{l}13249 . \\
77761 .\end{array}$ \\
\hline $\begin{array}{l}\text { COAL nEW } \\
\text { CAPACITY (MW) } \\
\text { GENERAT. (GWH) }\end{array}$ & $\begin{array}{l}0 . \\
0 .\end{array}$ & $\begin{array}{l}0 . \\
0 .\end{array}$ & $\begin{array}{r}1674 . \\
10997\end{array}$ & $\begin{array}{r}7480 . \\
49142 \text {. }\end{array}$ & $\begin{array}{l}14806 . \\
97276 .\end{array}$ \\
\hline $\begin{array}{l}\text { HYDRO } \\
\text { CAPACITY (MW) } \\
\text { GENERAT. (GWH) }\end{array}$ & $\begin{array}{l}2758 \\
6519\end{array}$ & $\begin{array}{l}2758 . \\
6765 .\end{array}$ & $\begin{array}{l}2758 . \\
6765 .\end{array}$ & $\begin{array}{l}2758 . \\
6765 .\end{array}$ & $\begin{array}{l}2758 . \\
6765 .\end{array}$ \\
\hline $\begin{array}{l}\text { OTHER } \\
\text { CAPAC!TY (MW) } \\
\text { GENERAT. (GWH) }\end{array}$ & $\begin{array}{l}134 . \\
1268 .\end{array}$ & $\begin{array}{l}344 . \\
904 .\end{array}$ & $\begin{array}{l}494 . \\
1298 .\end{array}$ & $\begin{array}{l}494 . \\
1298 .\end{array}$ & $\begin{array}{l}494 . \\
1298 .\end{array}$ \\
\hline $\begin{array}{l}\text { NUGS } \\
\text { CAPACITY (MW) } \\
\text { GENERAT. (GWH) }\end{array}$ & $\begin{array}{c}77 . \\
197 .\end{array}$ & $\begin{array}{l}3687 \\
25838\end{array}$ & $\begin{array}{r}3687 \\
25838\end{array}$ & $\begin{array}{l}3687 \\
25838\end{array}$ & $\begin{array}{r}3687 . \\
25838 .\end{array}$ \\
\hline $\begin{array}{l}\text { IMPORTS FOSSIL } \\
\text { CAPACITY (MH) } \\
\text { GENERAT. (GWH) }\end{array}$ & $\begin{array}{r}187 . \\
23273 .\end{array}$ & $\begin{array}{l}836 \\
7250\end{array}$ & $\begin{array}{l}716 . \\
6209\end{array}$ & $\begin{array}{r}716 . \\
6209\end{array}$ & $\begin{array}{r}716 . \\
6209 .\end{array}$ \\
\hline $\begin{array}{l}\text { NGCC neN } \\
\text { CAPACITY (MH) } \\
\text { GENERAT. (GWH) }\end{array}$ & $\begin{array}{l}0 . \\
0 .\end{array}$ & $\begin{array}{l}100 . \\
569 .\end{array}$ & $\begin{array}{r}570 . \\
3246 .\end{array}$ & $\begin{array}{l}2801 . \\
15949 .\end{array}$ & $\begin{array}{r}3777 . \\
21506 .\end{array}$ \\
\hline $\begin{array}{l}\text { NGCT neW } \\
\text { CAPACITY (MH) } \\
\text { GENERAT. (GWH) }\end{array}$ & $\begin{array}{l}0 . \\
0 .\end{array}$ & $\begin{array}{l}4221 . \\
825 .\end{array}$ & $\begin{array}{c}10258 . \\
8986 .\end{array}$ & $\begin{array}{l}11821 . \\
10355 .\end{array}$ & $\begin{array}{l}16014 . \\
14029 .\end{array}$ \\
\hline $\begin{array}{l}\text { DIST CT neH } \\
\text { CAPAC!TY (MH) } \\
\text { GENERAT. (GWH) }\end{array}$ & 0. & $\begin{array}{l}1168 \\
1023\end{array}$ & $\begin{array}{l}1793 . \\
1571 .\end{array}$ & $\begin{array}{l}1793 . \\
1571 .\end{array}$ & $\begin{array}{l}1793 . \\
1571 .\end{array}$ \\
\hline $\begin{array}{l}\text { WOOO } \\
\text { CAPACITY (MH) } \\
\text { GENERAT. (GWH) }\end{array}$ & $\begin{array}{l}\pi \\
446 .\end{array}$ & $\begin{array}{l}210 . \\
1196 .\end{array}$ & $\begin{array}{l}344 . \\
1959 .\end{array}$ & $\begin{array}{l}477 . \\
2716 .\end{array}$ & $\begin{array}{l}477 . \\
2716 .\end{array}$ \\
\hline $\begin{array}{l}\text { MSH } \\
\text { CAPACITY (MH) } \\
\text { GENERAT. (GWH) }\end{array}$ & $\begin{array}{l}104 . \\
603\end{array}$ & $\begin{array}{l}324 \\
1845\end{array}$ & $\begin{array}{r}544 . \\
3098 .\end{array}$ & $\begin{array}{r}544 . \\
3098 .\end{array}$ & $\begin{array}{l}544 . \\
3098 .\end{array}$ \\
\hline $\begin{array}{l}\text { LANDFILL GAS } \\
\text { CAPACITY (MH) } \\
\text { GENERAT. (GHH) }\end{array}$ & $\begin{array}{r}31 . \\
249 . \\
\end{array}$ & $\begin{array}{l}39 . \\
244:\end{array}$ & $\begin{array}{r}31 . \\
244 \\
-\end{array}$ & $\begin{array}{r}31 . \\
244 . \\
\end{array}$ & $\begin{array}{r}31 . \\
244 .\end{array}$ \\
\hline $\begin{array}{c}\text {-TOTAL ELECTRIC- } \\
\text { CAPACITY (MH) } \\
\text { GENERAT. (GWH) } \\
\text { RESERVE MARGIN(MH) }\end{array}$ & $\begin{array}{r}55581 . \\
255360 \\
5234\end{array}$ & $\begin{array}{r}67587 . \\
295908 . \\
11264 .\end{array}$ & $\begin{array}{l}74277 \\
336456 \\
12380\end{array}$ & $\begin{array}{r}81510 \\
378480 \\
13585\end{array}$ & $\begin{array}{r}91506 . \\
424896 . \\
15251 .\end{array}$ \\
\hline
\end{tabular}


Table 3.10

Reference Case

Electric Generation Projections

New York

\begin{tabular}{|c|c|c|c|c|c|}
\hline PLANT TYPE & 1985 & 1996 & 2004 & 2012 & 2020 \\
\hline $\begin{array}{l}\text { COAL STEAM } \\
\text { CAPACITY (MW) } \\
\text { GENERAT. (GWH) }\end{array}$ & $\begin{array}{r}4814 \\
26609\end{array}$ & $\begin{array}{r}4814 . \\
52471 .\end{array}$ & $\begin{array}{r}4814 \\
32471\end{array}$ & $\begin{array}{r}4814 . \\
32471 .\end{array}$ & $\begin{array}{r}4814 . \\
32471 .\end{array}$ \\
\hline $\begin{array}{l}\text { NG STEAM } \\
\text { CAPACITY (MW) } \\
\text { GENERAT. (GWH) }\end{array}$ & $\begin{array}{l}3644 . \\
13434 .\end{array}$ & $\begin{array}{l}3644 . \\
15961 .\end{array}$ & $\begin{array}{r}2744 . \\
12019 .\end{array}$ & $\begin{array}{l}1844 . \\
8077 .\end{array}$ & $\begin{array}{r}944 . \\
4135 .\end{array}$ \\
\hline $\begin{array}{l}\text { RESID STEAM } \\
\text { CAPACITY (MW) } \\
\text { GENERAT. (GWH) }\end{array}$ & $\begin{array}{l}9754 . \\
38921 \text {. }\end{array}$ & $\begin{array}{r}9754 . \\
31982 .\end{array}$ & $\begin{array}{r}7354 . \\
32211 .\end{array}$ & $\begin{array}{r}4954 . \\
21699\end{array}$ & $\begin{array}{r}2554 . \\
11187 .\end{array}$ \\
\hline $\begin{array}{l}\text { NG COMB TURB } \\
\text { CAPACITY (MW) } \\
\text { GENERAT. (GWH) }\end{array}$ & $\begin{array}{l}894 . \\
648 .\end{array}$ & $\begin{array}{l}894 . \\
392 .\end{array}$ & $\begin{array}{l}894 . \\
392 .\end{array}$ & $\begin{array}{l}894 . \\
392 .\end{array}$ & $\begin{array}{l}894 . \\
392 .\end{array}$ \\
\hline $\begin{array}{l}\text { DISTILLATE CT } \\
\text { CAPACITY (MH) } \\
\text { GENERAT. (GHH) }\end{array}$ & $\begin{array}{r}2684 . \\
780\end{array}$ & $\begin{array}{l}2924 . \\
1281 .\end{array}$ & $\begin{array}{l}2924 . \\
1281 .\end{array}$ & $\begin{array}{l}2924 . \\
1281 .\end{array}$ & $\begin{array}{l}2924 . \\
1281 .\end{array}$ \\
\hline $\begin{array}{l}\text { NUCLEAR } \\
\text { CAPACITY (MW) } \\
\text { GENERAT. (GWH) }\end{array}$ & $\begin{array}{r}4774 . \\
23617\end{array}$ & $\begin{array}{r}5583 \\
34235\end{array}$ & $\begin{array}{r}5583 \\
34235\end{array}$ & $\begin{array}{r}5583 . \\
34235 \text {. }\end{array}$ & $\begin{array}{r}5583 . \\
34235 .\end{array}$ \\
\hline $\begin{array}{l}\text { PUMP HYDRO } \\
\text { CAPACITY (MW) } \\
\text { GENERAT. (GWH) } \\
\text { HYDRO }\end{array}$ & $\begin{array}{l}1000 \\
1233\end{array}$ & $\begin{array}{l}1000 \\
263\end{array}$ & $\begin{array}{l}1000 \\
263\end{array}$ & $\begin{array}{r}1000 \\
263\end{array}$ & $\begin{array}{l}1000 . \\
263 .\end{array}$ \\
\hline $\begin{array}{l}\text { CAPACITY (MW) } \\
\text { GENERAT. (GWH) }\end{array}$ & $\begin{array}{r}3987 \\
24621\end{array}$ & $\begin{array}{r}4385 \\
24968\end{array}$ & $\begin{array}{r}4385 \\
24968\end{array}$ & $\begin{array}{r}4385 \\
24968\end{array}$ & $\begin{array}{r}4385 . \\
24968 .\end{array}$ \\
\hline $\begin{array}{l}\text { NUGS } \\
\text { CAPACITY (MW) } \\
\text { GENERAT. (GWH) }\end{array}$ & $\begin{array}{l}302 . \\
49 .\end{array}$ & $\begin{array}{l}302 . \\
1323 .\end{array}$ & $\begin{array}{l}302 \\
1323\end{array}$ & $\begin{array}{l}302 . \\
1323 .\end{array}$ & $\begin{array}{l}302 \\
1323\end{array}$ \\
\hline $\begin{array}{l}\text { IMPORTS FOSSIL } \\
\text { CAPACITY (MW) } \\
\text { GENERAT. (GWH) }\end{array}$ & $\begin{array}{l}77 . \\
1969 .\end{array}$ & $\begin{array}{l}94 . \\
815 .\end{array}$ & $\begin{array}{r}367 \\
3183\end{array}$ & $\begin{array}{r}367 . \\
3183 .\end{array}$ & $\begin{array}{r}367 . \\
3183 .\end{array}$ \\
\hline $\begin{array}{l}\text { NGCT nEW } \\
\text { CAPACITY (MH) } \\
\text { GENERAT. (GWH) }\end{array}$ & $\begin{array}{l}0 . \\
0 .\end{array}$ & 0. & $\begin{array}{l}1275 \\
558 .\end{array}$ & $\begin{array}{l}2521 . \\
1104 .\end{array}$ & $\begin{array}{l}4231 . \\
1853 .\end{array}$ \\
\hline $\begin{array}{l}\text { NUGS nEW } \\
\text { CAPACITY (MW) } \\
\text { GENERAT. (GWH) }\end{array}$ & $\begin{array}{l}0 . \\
0 .\end{array}$ & $\begin{array}{l}2671 \\
11699\end{array}$ & $\begin{array}{l}2716 . \\
11896\end{array}$ & $\begin{array}{l}2716 . \\
11896\end{array}$ & $\begin{array}{r}2716 . \\
11896 .\end{array}$ \\
\hline $\begin{array}{l}\text { NGCC neH } \\
\text { CAPACITY (MH) } \\
\text { GENERAT. (GHH) }\end{array}$ & 0. & $\begin{array}{l}0 . \\
0 .\end{array}$ & $\begin{array}{r}2382 \\
13563\end{array}$ & $\begin{array}{r}8522 . \\
48523 .\end{array}$ & $\begin{array}{l}15151 . \\
86268 .\end{array}$ \\
\hline $\begin{array}{l}\text { WOOO } \\
\text { CAPACITY (MH) } \\
\text { GEHERAT. (GWH) }\end{array}$ & $\begin{array}{l}13 . \\
74 .\end{array}$ & $\begin{array}{l}146 . \\
831 .\end{array}$ & $\begin{array}{l}280 . \\
1594 .\end{array}$ & $\begin{array}{l}413 . \\
2352\end{array}$ & $\begin{array}{l}413 . \\
2352 .\end{array}$ \\
\hline $\begin{array}{l}\text { MSW } \\
\text { CAPACITY (MH) } \\
\text { GENERAT. (GHH) }\end{array}$ & $\begin{array}{l}191 . \\
1337\end{array}$ & $\begin{array}{l}224 . \\
1570\end{array}$ & $\begin{array}{r}257 . \\
1801 .\end{array}$ & $\begin{array}{r}257 . \\
1801\end{array}$ & $\begin{array}{r}257 \\
1801 .\end{array}$ \\
\hline $\begin{array}{l}\text { LANDFILL } \\
\text { CAPACITY (MH) } \\
\text { GENERAT. (GHH) }\end{array}$ & $\begin{array}{l}31 . \\
244 .\end{array}$ & $\begin{array}{l}31 . \\
244 .\end{array}$ & $\begin{array}{l}31 . \\
244 .\end{array}$ & $\begin{array}{l}31 . \\
244 .\end{array}$ & $\begin{array}{l}31 . \\
244 .\end{array}$ \\
\hline $\begin{array}{l}\text { IMPORTS HYORO } \\
\text { CAPACI ITY (MH) } \\
\text { GENERAT. (GHH) }\end{array}$ & $\begin{array}{l}233 . \\
5905 \\
0\end{array}$ & $\begin{array}{r}285 \\
2472 \\
-\end{array}$ & $\begin{array}{l}1103 . \\
9566 \\
\end{array}$ & $\begin{array}{l}1103 . \\
9566 .\end{array}$ & $\begin{array}{l}1103 . \\
9566 .\end{array}$ \\
\hline $\begin{array}{l}\text { - TOTAL ELECTRIC- } \\
\text { CAPACITY (MH) } \\
\text { GENERAT. (GWH) } \\
\text { RESERVE MARGIN(MW) }\end{array}$ & $\begin{array}{r}32398 \\
139442 \\
7193\end{array}$ & $\begin{array}{r}36751 . \\
160506 . \\
8101 .\end{array}$ & $\begin{array}{r}38411 . \\
181567 \\
6402\end{array}$ & $\begin{array}{r}42630 \\
203376 \\
7105\end{array}$ & $\begin{array}{r}47669 . \\
227417 . \\
7945 .\end{array}$ \\
\hline
\end{tabular}




\section{Energy Related Emissions Factors}

Carbon dioxide and methane emissions factors were developed for each stage in the energy supply/demand path. Fuels directly combusted at the end-use (e.g., oil, natural gas, wood, landfill gas) were assigned emission factors at that point. Others fuels such as steam from MSW or electricity were assigned emission factors at the point of combustion, with each plant type given its specific factor.

Landfill gas from municipal solid waste was assumed to have a 20 percent degradable carbon content, 80 percent of which would degrade anaerobically over about 25 years. The emissions content of landfill gas is 50 percent $\mathrm{CO}_{2}$ and 50 percent $\mathrm{CH}_{4}$ on a molar basis, or about 75 percent $\mathrm{CO}_{2}$ and 25 percent $\mathrm{CH}_{4}$ on a mass basis. Those emissions not captured for energy use were estimated, changing over time as population growth and consumption change.

Emissions from oil refining, and oil, gas and coal extraction were also estimated, changing over time as demands for these primary fuels change. Emissions from decay of paper and pulp products were not separately estimated as these were assumed to be embodied in landfill gases.

Emission coefficients were obtained from a variety of sources, including the EPA's Atmospheric Stabilization Framework (ASF, 1990), the IPCC (IPCC, 1990), the Stockholm Environment Institute-Boston Center (SEI, 1990), and the Tellus Institute (Tellus, 1990), the OECD (OECD, 1990), and other international sources.

The relative global warming potentials of carbon dioxide and methane - their time integrated contribution to average temperature increases - increase the significance of the latter, which on simple mass terms alone contributes almost two orders of magnitude less than the former (expressed in carbon content). The GWPs depend upon the relative instantaneous radiative forcing of the different molecules $\mathrm{CO}_{2}$ and $\mathrm{CH}_{4}$ - their residence times in the atmosphere, the time period over which the forcings are integrated, and other factors. Here, we have chosen a GWP of 10.0 for methane -1.0 tons of $\mathrm{CH}_{4}$ is equivalent to 10.0 tons of $\mathrm{CO}_{2}$ or 2.7 tons of $\mathrm{C}$ in $\mathrm{CO}_{2}-$ from the work of Lashof and Ahuja (1990), assuming 100 years integration. ${ }^{23}$

\section{Reference Case Energy Emissions Results}

Table 3.11 is a summary of the carbon dioxide and methane emissions from energy use for the Reference Case. There is an overall increase in $\mathrm{CO}_{2}$ by about 27

\footnotetext{
${ }^{20}$ For 100 years the range of values in carbon dioxide equivalents was 22 to 36 . The IPCC gives 21 for the same time frame. The results are very sensitive to time frame. E.g, the IPCC gives 9,21 , and 63 for 500 years, 100 years and 20 years respectively.
} 
percent, and in $\mathrm{CH}_{4}$ by about 37 percent (owing in large measure to its increased use of coal (with associated methane leaks at mines).

Table 3.11

Reference Case, CONEG Energy System

Greenhouse Gas Emissions

(Million Tons)

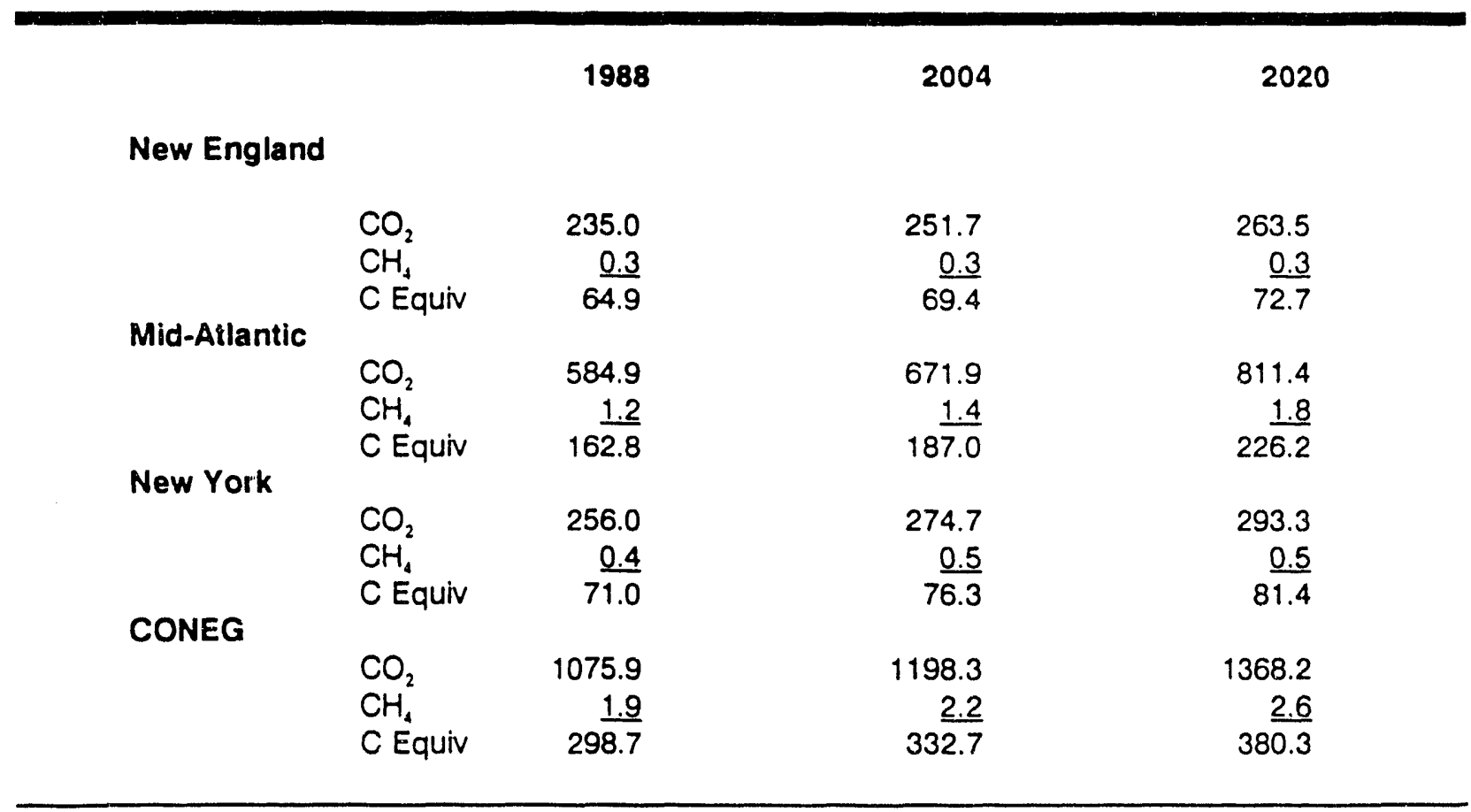

Contributions from end-use fuel combustion to overall $\mathrm{CO}_{2}$ emissions are over 60 percent - 64 for New England, 62 for Mid-Atlantic, and 64 for New York - and contributions from electricity generation are about 30 percent -29 for New England, 34 for Mid-Atlantic, and 30 for New York. The rest come from MSW combustion and other transformation processes such as oil refining. The contribution from end-use decreases over time, with the difference taken up by increases in electricity's contribution. The maximum shift is about 8 percentage points by 2020 for the MidAtlantic, owing to its greater increase in coal use.

Methane is released almost entirely in the energy transformation processes, including leakages in natural gas distribution and leakages from coal mine walls. 


\subsection{Conservation Case Energy Projections}

As a context for our examination of the potential role of biomass in mitigating greenhouse gas emissions in the region we assumed that significant levels of end-use energy efficiency would be realized across all sectors over the period 1988 to 2020 . The levels of conservation assumed here are indicative, and not based upon a detailed analysis of the potential in the region. We used estimates - in the range of 20 to 35 percent reductions relative to Reference Case energy use by 2020 - based in part on the results of a recent national study, America's Energy Choices (Meyer, 1991).

In this initial conservation scenario, no modification is assumed in biomass supply. End-use biomass demand, however, is assumed to be reduced as a result of conservation. It will be apparent that while energy conservation and renewable energy supplies generally will both likely contribute to national and regional energy and greenhouse gas strategies, biomass alone will likely play an important but not a dominant role.

\section{Conservation Case Energy Results}

Tables 3.12 a,b, and c provide summary results of our Conservation Case enduse energy projections for New England, the Mid-Atlantic, and New York, respectively. The overall level of end-use energy conservation assumed is about 30 percent by 2020 , about 1.2 percent per year. The study for which these conservation estimates were obtained (Meyer, 1991) found that these levels of conservation could be realized at or below avoided fuel costs (i.e. at no net cost). Greater levels -- up to about 45 percent overall -- could be achieved at net costs below about $\$ 25$ per ton of carbon dioxide reduction. Tables $3.13,3.14$ and 3.15 provide the modified electric supply system consistent with these levels of demand reductions. These modifications entail reduced construction and operation of new baseload coal and natural gas combined cycle facilities and new gas turbine peakers. 
Table $3.12 a, b, c$

Conservation Case: End-Use Energy Projections

Trillion Btu

\begin{tabular}{|c|c|c|c|c|c|}
\hline & 1988 & 1996 & 2004 & 2012 & 2020 \\
\hline \multicolumn{6}{|c|}{ a. New England } \\
\hline Electricity & 349.2 & 380.6 & 399.0 & 412.6 & 431.7 \\
\hline Natural Gas & 311.7 & 307.9 & 297.7 & 285.6 & 275.3 \\
\hline Oil & 1521.4 & 1378.3 & 1215.5 & 1073.7 & 91.2 \\
\hline Coal & 17.8 & 18.3 & 18.4 & 19.1 & 21.6 \\
\hline Renewable & 271.8 & 266.9 & 260.6 & 257.9 & 266.6 \\
\hline Total New Eng & 2471.9 & 2351.9 & 2191.2 & 2049.0 & 1986.4 \\
\hline Connecticut & 553.9 & 529.9 & 495.6 & 464.7 & 451.6 \\
\hline Maine & 401.6 & 381.7 & 357.2 & 338.5 & 339.2 \\
\hline Massachusetts & 1020.6 & 962.5 & 887.5 & 817.9 & 773.8 \\
\hline New Hamphire & 211.7 & 207.2 & 198.6 & 191.4 & 191.5 \\
\hline Rhode Island & 160.3 & 149.6 & 136.6 & 125.4 & 120.6 \\
\hline Vermont & 124.0 & 121.0 & 115.8 & 111.0 & 109.6 \\
\hline \multicolumn{6}{|c|}{ b. Mid-Atlantic } \\
\hline Electricity & 785.1 & 849.1 & 886.5 & 915.5 & 959.6 \\
\hline Natural Gas & 1262.1 & 1223.0 & 1164.2 & 1110.8 & 1088.0 \\
\hline Oil & 2888.3 & 2689.8 & 2439.4 & 2213.5 & 2086.7 \\
\hline Coal & 495.4 & 523.7 & 542.1 & 574.5 & 658.3 \\
\hline Renewable & 174.0 & 171.6 & 168.0 & 166.1 & 170.1 \\
\hline Total Mid-Atl & 5604.9 & 5457.2 & 5200.3 & 4980.5 & 4962.7 \\
\hline Delaware & 175.3 & 173.2 & 167.2 & 162.1 & 163.3 \\
\hline Maryland & 869.8 & 861.4 & 832.3 & 809.8 & 826.3 \\
\hline New Jersey & 1781.1 & 1693.8 & 1575.3 & 1469.8 & 1421.9 \\
\hline Pennsylvania & 2778.5 & 2728.9 & 2625.4 & 2538.8 & 2551.3 \\
\hline \multicolumn{6}{|c|}{ c. New York } \\
\hline Electricity & 428.7 & 459.7 & 476.0 & 487.1 & 504.0 \\
\hline Natural Gas & 660.1 & 640.6 & 609.0 & 576.4 & 553.0 \\
\hline Oil & 1381.9 & 1225.1 & 1055.7 & 910.9 & 825.0 \\
\hline Coal & 99.2 & 104.5 & 108.0 & 114.3 & 131.4 \\
\hline Renewable & 120.4 & 118.9 & 116.7 & 115.3 & 117.8 \\
\hline Total New York & 2690.3 & 2548.8 & 2365.3 & 2204.2 & 2131.2 \\
\hline
\end{tabular}


Table 3.13

Conservation Case

\section{Electric Generation Projections}

New England

\begin{tabular}{|c|c|c|c|c|c|}
\hline PLANT TYPE & 1988 & 1996 & 2004 & 2012 & 2020 \\
\hline $\begin{array}{l}\text { COAL STEAM } \\
\text { CAPACITY (MW) } \\
\text { GENERAT. (GWH) }\end{array}$ & $\begin{array}{l}2751 \\
15002\end{array}$ & $\begin{array}{l}2751 . \\
15664 .\end{array}$ & $\begin{array}{l}2751 . \\
15664 .\end{array}$ & $\begin{array}{l}2751 . \\
15664 .\end{array}$ & $\begin{array}{r}2751 . \\
15664 .\end{array}$ \\
\hline $\begin{array}{l}\text { RESID STEAM } \\
\text { CAPACITY (MH) } \\
\text { GENERAT. (GWH) }\end{array}$ & $\begin{array}{r}8381 \\
37066\end{array}$ & $\begin{array}{r}8062 \\
26798\end{array}$ & $\begin{array}{r}5962 . \\
30108 .\end{array}$ & $\begin{array}{r}3862 . \\
20299\end{array}$ & $\begin{array}{l}1762 . \\
9261 .\end{array}$ \\
\hline $\begin{array}{l}\text { NG COMB CYCLE } \\
\text { CAPACITY (MH) } \\
\text { GENERAT. (GWH) }\end{array}$ & $\begin{array}{l}146 . \\
563 .\end{array}$ & $\begin{array}{l}146 . \\
831 .\end{array}$ & $\begin{array}{l}146 . \\
831 .\end{array}$ & $\begin{array}{l}146 . \\
831 .\end{array}$ & $\begin{array}{l}146 . \\
831 .\end{array}$ \\
\hline $\begin{array}{l}\text { OISTILLATE CC } \\
\text { CAPACITY (MH) } \\
\text { GENERAT. (GWH) }\end{array}$ & $\begin{array}{l}311 . \\
734 .\end{array}$ & $\begin{array}{l}311 . \\
1226 .\end{array}$ & $\begin{array}{l}311 . \\
1226 .\end{array}$ & $\begin{array}{l}311 . \\
1226 .\end{array}$ & $\begin{array}{l}311 . \\
1226 .\end{array}$ \\
\hline $\begin{array}{l}\text { NG COMB TURB } \\
\text { CAPACITY (MH) } \\
\text { GENERAT. (GWH) }\end{array}$ & $\begin{array}{l}89 . \\
40 .\end{array}$ & $\begin{array}{l}89 . \\
39 .\end{array}$ & $\begin{array}{l}89 . \\
39 .\end{array}$ & $\begin{array}{l}89 . \\
39 .\end{array}$ & $\begin{array}{l}89 . \\
39 .\end{array}$ \\
\hline $\begin{array}{l}\text { DISTILLATE } \\
\text { CT } \\
\text { CAPACITY } \\
\text { GENERAT. } \\
\text { GH) }\end{array}$ & $\begin{array}{r}1262 . \\
453 .\end{array}$ & $\begin{array}{c}1070 . \\
469 .\end{array}$ & $\begin{array}{l}981 . \\
430 .\end{array}$ & $\begin{array}{l}981 . \\
430 .\end{array}$ & $\begin{array}{l}981 . \\
430 .\end{array}$ \\
\hline $\begin{array}{l}\text { NUCLEAR } \\
\text { CAPACITY (MW) } \\
\text { GENERAT. (GWH) }\end{array}$ & $\begin{array}{r}4737 \\
32751\end{array}$ & $\begin{array}{r}5887 \\
38678\end{array}$ & $\begin{array}{r}5887 \\
38678\end{array}$ & $\begin{array}{l}5887 \\
38678\end{array}$ & $\begin{array}{r}5887 \\
38678 .\end{array}$ \\
\hline $\begin{array}{l}\text { PUMP HYDRO } \\
\text { CAPACITY (MH) } \\
\text { GENERAT. (GWH) }\end{array}$ & $\begin{array}{l}1594 . \\
1640 .\end{array}$ & $\begin{array}{l}1594 . \\
1396 .\end{array}$ & $\begin{array}{l}1594 . \\
1396 .\end{array}$ & $\begin{array}{l}1594 . \\
1396 .\end{array}$ & $\begin{array}{l}1594 . \\
1396 .\end{array}$ \\
\hline $\begin{array}{l}\text { HYORO } \\
\text { CAPACITY (MH) } \\
\text { GENERAT. (GWH) }\end{array}$ & $\begin{array}{l}1382 . \\
4446 .\end{array}$ & $\begin{array}{l}1474 . \\
5165 .\end{array}$ & $\begin{array}{l}1474 . \\
5165^{\circ}\end{array}$ & $\begin{array}{l}1474 . \\
5165 .\end{array}$ & $\begin{array}{l}1474 . \\
5165 .\end{array}$ \\
\hline $\begin{array}{l}\text { OTHER } \\
\text { CAPACITY (MH) } \\
\text { GENERAT. (GWH) }\end{array}$ & $\begin{array}{l}0 . \\
0 .\end{array}$ & $\begin{array}{l}10 . \\
44 .\end{array}$ & $\begin{array}{l}10 . \\
44 .\end{array}$ & $\begin{array}{l}10 . \\
44 .\end{array}$ & $\begin{array}{l}10 . \\
44 .\end{array}$ \\
\hline $\begin{array}{l}\text { NUGS } \\
\text { CAPACITY (MH) } \\
\text { GENERAT. (GWH) }\end{array}$ & $\begin{array}{l}0 . \\
0 .\end{array}$ & $\begin{array}{l}888 . \\
4667 .\end{array}$ & $\begin{array}{l}888 . \\
4667\end{array}$ & $\begin{array}{l}888 . \\
4667\end{array}$ & $\begin{array}{l}888 . \\
4667\end{array}$ \\
\hline $\begin{array}{l}\text { IMPORTS FOSSIL } \\
\text { CAPACITY (MH) } \\
\text { GENERAT. (GWH) }\end{array}$ & $\begin{array}{l}201 . \\
3138 .\end{array}$ & $\begin{array}{l}303 . \\
2628\end{array}$ & $\begin{array}{l}476 . \\
4128\end{array}$ & $\begin{array}{l}476 . \\
4128 .\end{array}$ & $\begin{array}{r}476 . \\
4128 .\end{array}$ \\
\hline $\begin{array}{l}\text { NGCC nEH } \\
\text { CAPACITY (MH) } \\
\text { GENERAT. (GWH) }\end{array}$ & $\begin{array}{l}0 . \\
0 .\end{array}$ & $\begin{array}{l}827 . \\
4709\end{array}$ & $\begin{array}{l}827 . \\
4709 .\end{array}$ & $\begin{array}{r}3649 . \\
20775\end{array}$ & $\begin{array}{l}7284 \\
41476\end{array}$ \\
\hline $\begin{array}{l}\text { NGCT neH } \\
\text { CAPACITY (MH) } \\
\text { GENERAT. (GWH) }\end{array}$ & $\begin{array}{l}0 . \\
0 .\end{array}$ & $\begin{array}{l}0 . \\
0 .\end{array}$ & $\begin{array}{r}2434 . \\
0 .\end{array}$ & $\begin{array}{l}3885 \\
1702\end{array}$ & $\begin{array}{l}4370 . \\
1914 .\end{array}$ \\
\hline $\begin{array}{l}\text { NUGS nEH } \\
\text { CAPACITY (MW) } \\
\text { GENERAT. (GWH) }\end{array}$ & $\begin{array}{l}0 . \\
0 .\end{array}$ & $\begin{array}{l}888 . \\
4667 \text {. }\end{array}$ & $\begin{array}{l}888 \text {. } \\
4667 \text {. }\end{array}$ & $\begin{array}{l}888 \text {. } \\
4667 \text {. }\end{array}$ & $\begin{array}{l}888 . \\
4667\end{array}$ \\
\hline $\begin{array}{l}\text { WOOO } \\
\text { CAPACITY (MH) } \\
\text { GENERAT. (GWH) }\end{array}$ & $\begin{array}{l}716 . \\
4098 .\end{array}$ & $\begin{array}{l}739 . \\
4208 .\end{array}$ & $\begin{array}{l}763 . \\
4345\end{array}$ & $\begin{array}{l}786 . \\
4475 .\end{array}$ & $\begin{array}{l}786 . \\
4475 .\end{array}$ \\
\hline $\begin{array}{l}\text { MSH } \\
\text { CAPACITY (MH) } \\
\text { GENERAT. (GWH) }\end{array}$ & $\begin{array}{l}470 . \\
2694 .\end{array}$ & $\begin{array}{r}520 . \\
2961 .\end{array}$ & $\begin{array}{c}570 . \\
3246 .\end{array}$ & $\begin{array}{r}570 . \\
3246 .\end{array}$ & $\begin{array}{l}570 . \\
3246 .\end{array}$ \\
\hline $\begin{array}{l}\text { LANDFILL GAS } \\
\text { CAPACITY (MH) } \\
\text { GENERAT. (GHH) }\end{array}$ & $\begin{array}{l}26 . \\
206 .\end{array}$ & $\begin{array}{l}26 . \\
205 .\end{array}$ & $\begin{array}{l}26 . \\
205 .\end{array}$ & $\begin{array}{l}26 . \\
205 .\end{array}$ & $\begin{array}{l}26 . \\
205 .\end{array}$ \\
\hline $\begin{array}{l}\text { IMPORTS HYORO } \\
\text { CAPACITY (MH) } \\
\text { GENERAT. (GWH) }\end{array}$ & $\begin{array}{l}602 . \\
9415\end{array}$ & $\begin{array}{l}906 . \\
7857 . \\
\end{array}$ & $\begin{array}{r}1425 . \\
12358 \\
\cdots \cdots\end{array}$ & $\begin{array}{r}1425 \\
12358 \\
\cdots\end{array}$ & $\begin{array}{r}1425 . \\
12358 . \\
\cdots\end{array}$ \\
\hline $\begin{array}{l}\text { - TOTAL ELECTRIC- } \\
\text { CAPACITY (MH) } \\
\text { GENERAT. (GWH) } \\
\text { RESERVE MARGIN(MH) }\end{array}$ & $\begin{array}{r}22947 . \\
113583 . \\
4188 .\end{array}$ & $\begin{array}{r}26790 . \\
123784 . \\
5813 .\end{array}$ & $\begin{array}{r}27101 . \\
129798 \\
4517\end{array}$ & $\begin{array}{l}28596 \\
134208 \\
4766\end{array}$ & $\begin{array}{r}29917 . \\
140405 . \\
4986 .\end{array}$ \\
\hline
\end{tabular}


Tatie 3.14

Conservation Case

Electric Generation Projections

Mid-Atlantic

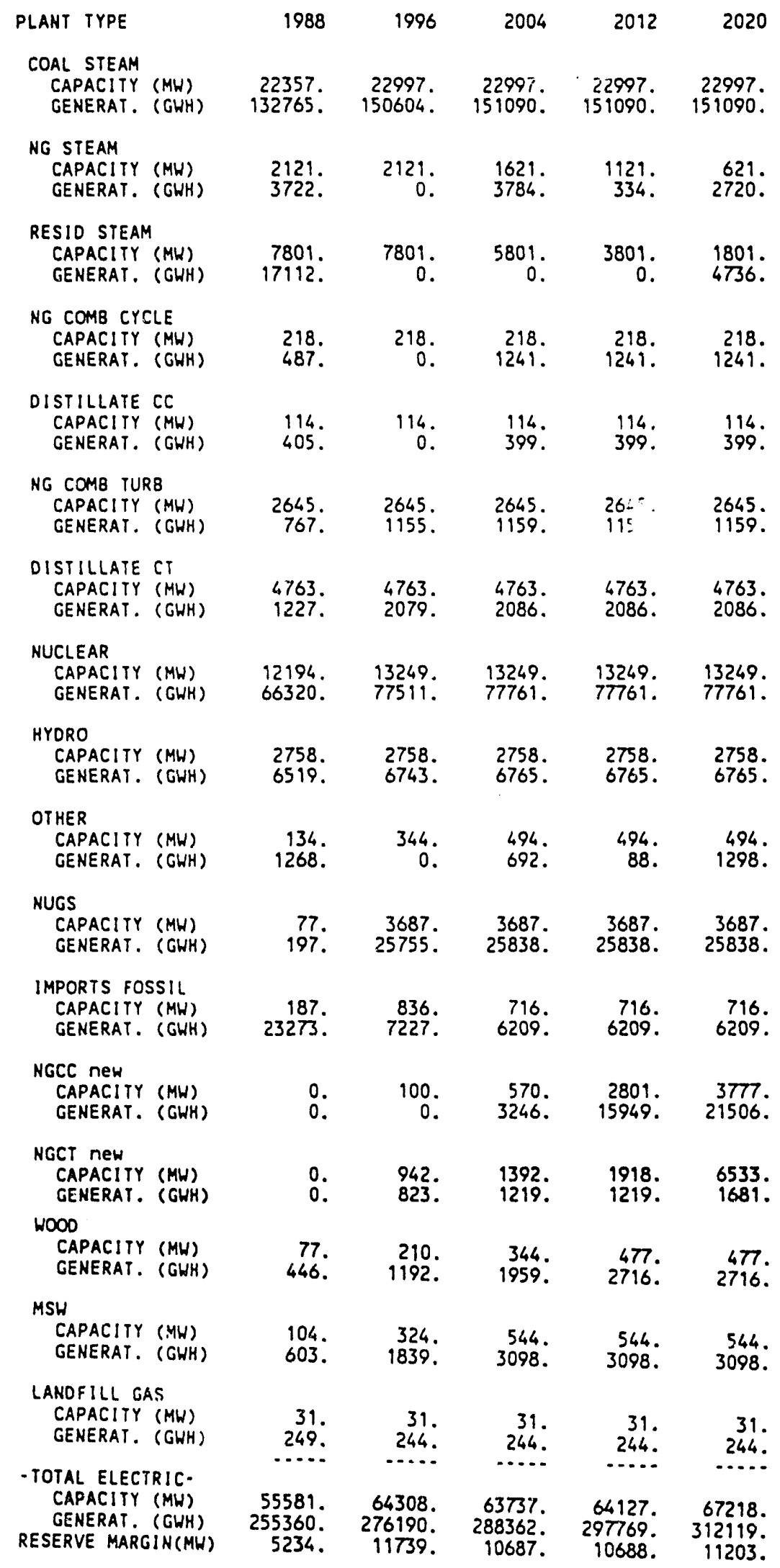


Table 3.15

Conservation Case

Electric Generation Projections

New York

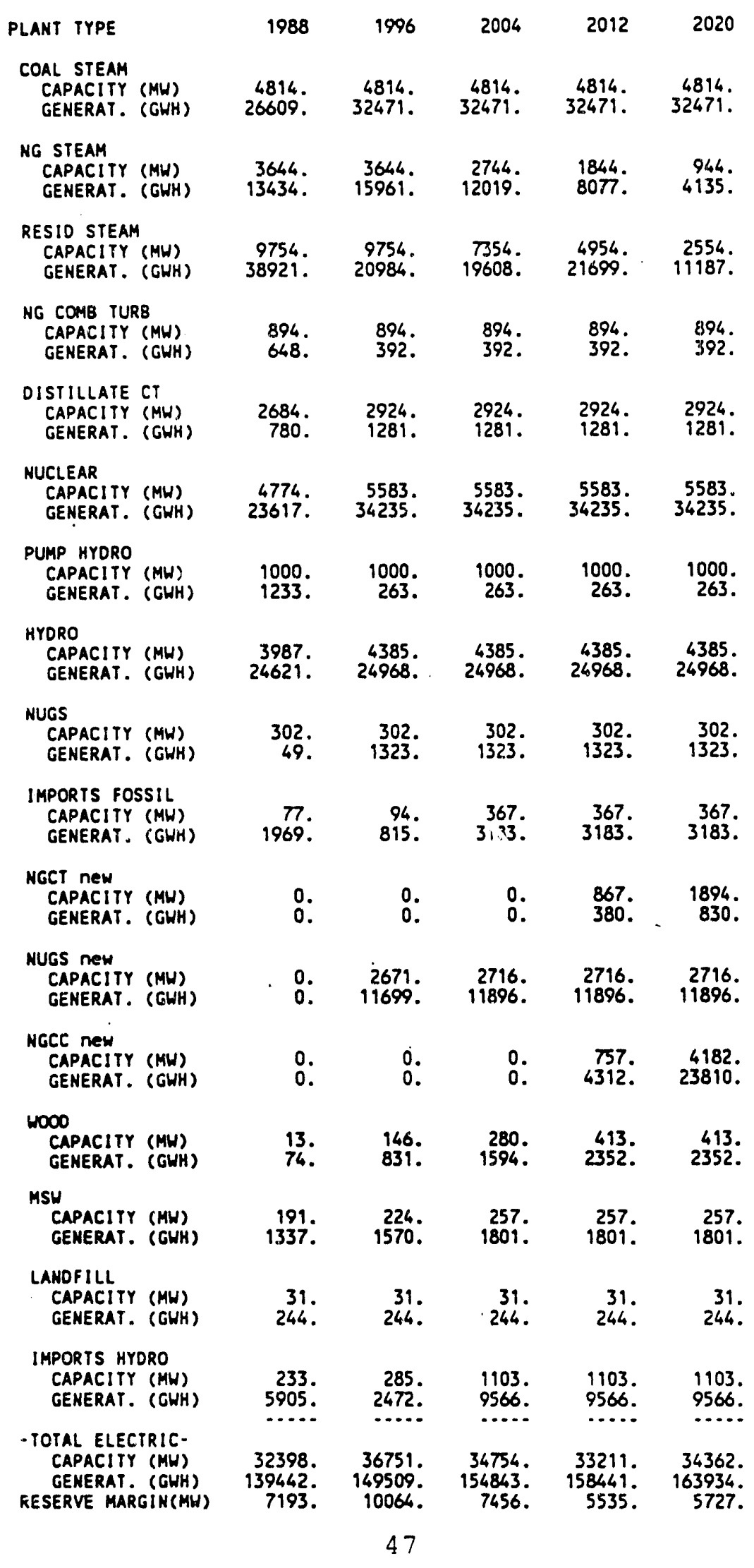




\section{Conservation Case Energy Emissions Results}

Table 3.16 summarizes the greenhouse gas emissions associated with the Conservation Case.

Table 3.16

Conservation Case, CONEG Energy System

Greenhouse Gas Emissions

(Million Tons)

\begin{tabular}{|c|c|c|c|c|}
\hline & & 1988 & 2004 & 2020 \\
\hline \multicolumn{5}{|l|}{ New England } \\
\hline & $\mathrm{CO}_{2}$ & 235.0 & 212.0 & 184.1 \\
\hline & $\begin{array}{l}\mathrm{CH}_{4} \\
\mathrm{C} \text { Equin }\end{array}$ & $\underline{0.3}$ & $\underline{0.3}$ & $\underline{0.3}$ \\
\hline \multicolumn{5}{|l|}{ Mid-Atlantic } \\
\hline & $\mathrm{CO}_{2}$ & 584.9 & 559.8 & 555.1 \\
\hline & $\mathrm{CH}_{4}$ & 1.2 & 1.3 & $\frac{1.3}{1.0}$ \\
\hline & C Equiv & 162.8 & 156.2 & 154.9 \\
\hline \multicolumn{5}{|l|}{ New York } \\
\hline & $\mathrm{CO}_{2}$ & 256.0 & 227.5 & 207.7 \\
\hline & $\begin{array}{l}\mathrm{CH}_{4} \\
\mathrm{C} \text { Equin }\end{array}$ & $\frac{0.4}{71.0}$ & $\underline{0.4}$ & $\begin{array}{r}0.4 \\
57.8\end{array}$ \\
\hline \multicolumn{5}{|l|}{ CONEG } \\
\hline & $\mathrm{CO}_{2}$ & 1075.9 & 999.3 & 946.9 \\
\hline & $\mathrm{CH}_{4}$ & 1.9 & $\underline{2.0}$ & 2.0 \\
\hline & C Equiv & $29 \overline{8.7}$ & $27 \overline{8.0}$ & $26 \overline{3.7}$ \\
\hline
\end{tabular}

The Conservation Case embodies across the board reductions in carbon dioxide and methane, amounting to about 30 percent decrease in carbon equivalent by 2020 relative to the Reference Case, and a 12 percent decline from 1988 levels. Energy conservation reduces combustion and emissions in both end-use demand and electricity generation. The greatest electric sector emissions decrease by 2020 is 35 percent in the Mid-Atlantic, owing principally to the reduction in coal-fired power generation.

\subsection{The CONEG States Solid Waste Systems}

The carbon releases from Municipal Solid '..ite (MSW) facilities and combustion of captured landfill gas are embodied he energy system emissions results presented earlier. Here we add the gross emissior. Im landfill decay and composting, taking account of the states' population growth, growin in consumption, and current plans to implement the more advanced solid waste management techniques. Later, in Section 6 we will present the strategy targets for reduction of solid waste greenhouse 
gas emissions from the levels given here. (A self contained treatment of all emissions and uptakes in the CONEG states' solid waste carbon cycles is given in Appendix A.)

Municipal solid waste is generated in the CONEG region in about equal amounts from the residential and commercial sectors, at about 1.2 tons per capita annually. We have assumed that population growth and a 1 percent annual increase in per capita solid waste generation will increase the magnitude of the solid waste stream over the thirty year study period. As noted earlier, solid waste can produce both carbon dioxide and methane, depending upon the mode of its management.

The municipal solid waste systems of the CONEG states can be managed through landfill, incineration, resource recovery (MSW), recycling, composting (of the organic fraction), and source reduction. Landfill and incineration are the more traditional modes of management, while resource recovery (incineration for energy production) emerged in the past two decades as a dual purpose - waste disposal and energy - option. The last three options - recycling, composting and source reduction are in their early stages, with the pressure of land scarcity and cost and urban environmental concerns stimulating policy and planning initiatives in the region.

Landfill carbon and methane releases are the residual emissions after source reduction, recycling, composting, and MSW resource recovery have removed portions of the solid waste stream. Some of the methane created in the landfill could then be captured and used as gaseous fuel, along with conventional natural gas, leaving the remainder of the methane to be released to the atmosphere.

The total residential and commercial solid waste generated in the CONEG region was about 57 million tons per year in 1988 (12.4 for New England, 24.5 for the Mid-Atlantic, and 20.3 for New York). Based on population growth and an assumed one percent annual increase in per-capita solid waste generation, this will increase by 43 percent to about 83 million tons per year by 2020 . If all of the waste degraded in landfill, the emissions would be about 19 million tons $\mathrm{CO}_{2}$ and 7 million tons $\mathrm{CH}_{4}$ in 1988. Assuming the carbon dioxide equivalent GWP for methane is 10 (2.73 in C equivalent), this is a total GWP of about 24.4 million tons carbon equivalent. This is about 8 percent of the total GWP of 298 million tons from energy related emissions given earlier. ${ }^{24}$ With the level of waste generation increasing by 43 percent by 2020 , the 24 million tons GWP would increase to about 35 million tons.

In fact, owing to existing solid waste management methods, including resource recovery, the total carbon equivalent emissions from the entire solid waste system was about 19 million tons in 1988 , or about 6.3 percent of the total carbon equivalent

24 With a higher methane GWP of 26 in $\mathrm{CO}_{2}$ equivalent or 7.1 in $\mathrm{C}$ equivalent, the total maximum GWP from landfill currently would be about 37.5 million tons carbon equivalent. 
emissions from energy. Given current plans and policies in the CONEG states - wasteto-energy resource recovery, recycling, composting, and source reduction - the solid waste system GWP should decline to about 13 million tons by 2020, despite population and consumption growth that would suggest a 43 percent increase to 27 million tons. Thus, changes in the solid waste management system will decrease carbon equivalent emissions to about one-half of what they would otherwise be by the year $2020 .^{25}$

In Table 3.17 below, is a summary of our Reference Case solid waste system landfill (and composting) emissions, based on a set of current state policies, recently summarized in the May 1991 issue of Biocycle (Glenn, 1991). ${ }^{26}$ The composting, recycling and source reduction goals embodied in these current state policies are generally quite ambitious. We modified them state by state to be less than or equal to our strategy goals (discussed in Section 6) of 20 percent composting, 40 percent recycling, and 20 percent source reduction. The year 2020 recycling targets embodied in our solid waste system Reference Case range from 15 percent (for Vermont) to 35 percent (for Maine) in New England, from 10 percent (for Delaware) to 40 percent (for New Jersey) in the Mid-Atlantic, and 40 percent for New York. While zero in some states, composting reaches a maximum of 20 percent (in Massachusetts and Delaware). The highest source reduction targets are taken to be 20 percent (for Connecticut, New Jersey and Pennsylvania).

We have assumed that current plans to build MSW resource recovery facilities before the end of the century are completed, and that no new landfill gas capture for energy production is added.

${ }^{25}$ It should be noted that if the higher value for the carbon equivalent of methane (7.1) were used, the carbon equivalent emissions from the CONEG state solid waste systems would be about 40 percent higher or about 9 percent of total current emissions from the energy system.

${ }^{20}$ A source reduction initiative adopted by CONEG in 1991 called for reduction in packaging waste to a level that contributes to the overall goal of at least 50 percent reduction in all solid waste in the Northeast by the year 2000. As a result, in 1992 the CONEG Source Reduction Task Force drafted model legislation that sets a 15 percent packaging reduction standard for 1996 and a 35 percent packaging reduction goal for the year 2000. Waste from packaging currently makes up about one-third of the municipal solid waste stream. Although the CONEG Source Reduction Initiative is indicative of the kind of plans and policies anticipated in this discussion, the source reduction projections used by Tellus to build the Reference Case and other scenarios were developed prior to adoption of this policy by the CONEG states, and do not correspond to the reduction targets set by this policy. 
Table 3.17

CONEG Region Solid Waste System

Greenhouse Gas Emissions

(Million Tons)

\section{Reference Case}

New England

Landfill Emissions

$\mathrm{CO} 2$
$\mathrm{CH} 4$
$\mathrm{C}$ Equin

$\begin{array}{lllll}1988 & 1996 \quad 2004 & 2012 & 2020\end{array}$

$\mathrm{CH} 4$

\begin{tabular}{lllll}
1.83 & 1.64 & 1.68 & 2.01 & 2.29 \\
0.75 & 0.42 & 0.12 & 0.18 & 0.24 \\
\hline 2.14 & 1.37 & 0.73 & 0.93 & 1.14
\end{tabular}

Res Recovery Emissions

$\mathrm{CO} 2$

$C$ Equin

\begin{tabular}{lllll}
4.65 & 5.67 & 5.93 & 5.93 & 5.93 \\
\hline 1.27 & 1.55 & 1.62 & 1.62 & 1.62
\end{tabular}

Total C Equivalent

$\begin{array}{lllll}3.41 & 2.91 & 2.34 & 2.55 & 2.76\end{array}$

Mid Allantic

Landfill Emissions

$\mathrm{CO} 2$
$\mathrm{CH}_{4}$
$\mathrm{C}$ Equin

\begin{tabular}{lllll}
6.53 & 4.19 & 3.79 & 3.73 & 3.78 \\
2.20 & 1.25 & 1.05 & 0.97 & 0.96 \\
\hline 7.79 & 4.54 & 3.90 & 3.68 & 3.64
\end{tabular}

Res Recovery Emissions

$\mathrm{CO} 2$

C Equin

\begin{tabular}{lllll}
3.44 & 6.87 & 6.87 & 6.87 & 6.87 \\
\hline 0.94 & 1.87 & 1.87 & 1.87 & 1.87
\end{tabular}

Tolal C Equivalent

$\begin{array}{lllll}8.73 & 6.41 & 5.77 & 5.55 & 5.51\end{array}$

New York

Landfill Emissions

$\mathrm{CO} 2$
$\mathrm{CH} 4$
$\mathrm{C}$ Equin

\begin{tabular}{rrrrr}
4.71 & 3.61 & 3.27 & 2.82 & 3.19 \\
1.71 & 1.31 & 1.19 & 1.03 & 1.16 \\
\hline 5.96 & 4.56 & 4.14 & 3.57 & 4.03
\end{tabular}

Res Recovery Emissions

$\mathrm{CO} 2$

C Equin

\begin{tabular}{lllll}
2.95 & 3.40 & 3.40 & 3.40 & 3.40 \\
\hline 0.80 & 0.93 & 0.93 & 0.93 & 0.93
\end{tabular}

Total C Equivalent

$\begin{array}{lllll}6.76 & 5.49 & 5.06 & 4.49 & 4.96\end{array}$

CONEG Region

Landfill Emission:

$\mathrm{CO} 2$
$\mathrm{CH} 4$
$\mathrm{C}$ Equin

\begin{tabular}{rrrrr}
13.07 & 9.44 & 8.74 & 8.56 & 9.25 \\
4.67 & 2.97 & 2.35 & 2.18 & 2.35 \\
\hline 15.89 & 10.47 & 8.76 & 8.17 & 8.81
\end{tabular}

Res Recovery Emissions

$\mathrm{CO} 2$

C Equin

\begin{tabular}{rrrrr}
11.03 & 15.94 & 16.20 & 16.20 & 16.20 \\
\hline 3.01 & 4.35 & 4.42 & 4.42 & 4.42 \\
18.90 & 14.81 & 13.17 & 12.59 & 13.23
\end{tabular}


Note that the total carbon equivalent emissions from the entire solid waste system was about 19 million tons in 1988, about 6.3 percent of total carbon equivalent emissions from energy. This declines to about 13 million tons by 2020 , despite population and consumption growth that would cause an increase to about 27 million. Thus, changes in the solid waste management system will decrease 2020 carbon equivalent emissions by about one-half of what they would otherwise be. Finally, it should be noted that if the higher value for the carbon equivalent of methane (7.1) were used, the carbon equivalent emissions from the CONEG states' solid waste systems would be about 50 percent higher, or about 9 percent of total emissions from the energy system.

\subsection{Total Emissions: Energy and Solid Waste}

\section{Reference Case}

In Tables 3.18 and 3.19 we combine the emissions results for the energy and solid waste systems in the region for the Reference Case and the Conservation Case, respectively.

In the Reference Case overall carbon equivalent emissions increase by about 24 percent over the 32 year period, with the Mid-Atlantic increasing by 35 percent owing in part to our assumption that new power supply would be primarily coal. 


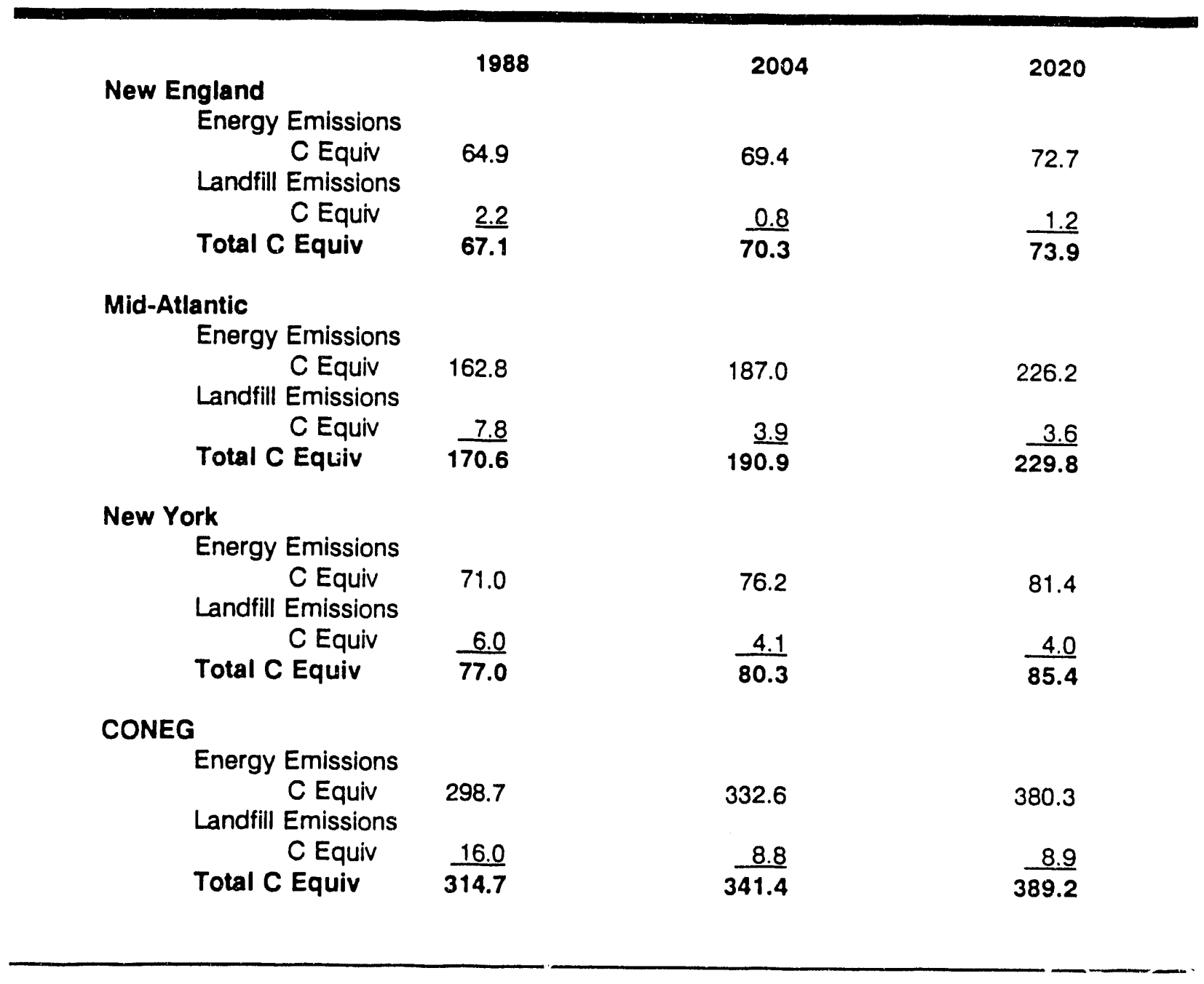

\section{Conservation Case}

In the Conservation Case overall carbon equivalent emissions decrease by about 13 percent over the 32 year period, rather than increase by 24 percent as they do in the Reference Case. By 2020, then, overall reductions achieved by energy conservation (and solid waste management) amount to about 30 percent. 
Table 3.19

Conservation Case Energy and Landfill Emissions

(Million Tons)

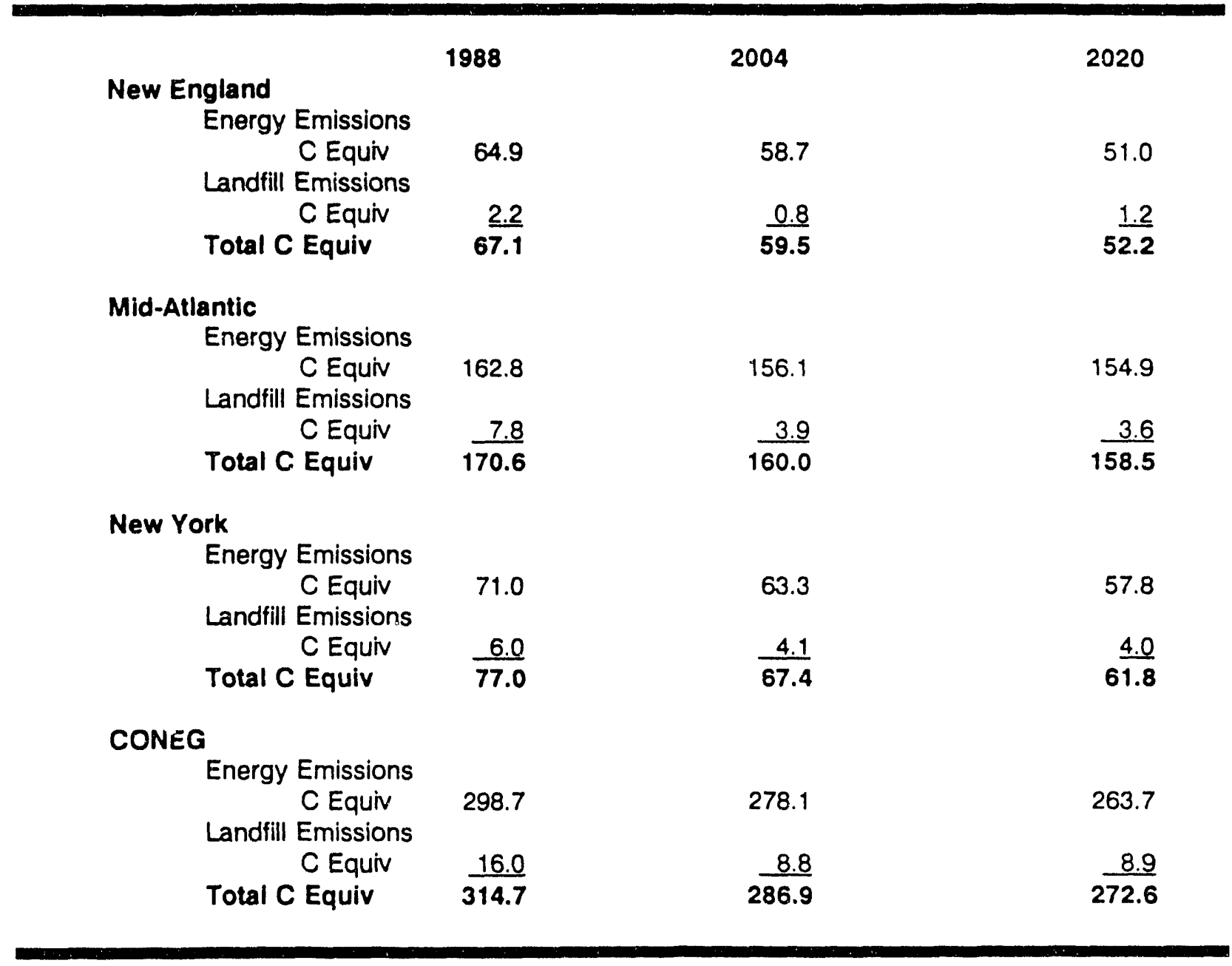

\subsection{Biomass Policy Scenarios}

The remainder of this report focuses on the role of biomass and its impact - and potential impact - on the region's net carbon equivalent emissions. Before proceeding to the policy scenarios, however, the next section is offered to establish the relevant methods and assumptions used in this study to estimate forest biomass and its impact on the region's carbon cycle, and to provide background on the forest characteristics and activities that could influence the potential scope of biomass strategies and policies. Section 5 then combines the carbon cycle impacts associated with Current Biomass Policy with the energy and solid waste systems impacts of the Reference Case and the Conservation Case, to produce Scenarios 1 and 2. Section 6 applies first Moderate Biomass Policy and then Advanced Biomass Policy to the energy and solid waste system impacts associated with the Conservation Case, resulting in Scenarios 3 and 4. Policy recommendations are presented in Section 7. 


\section{Accounting for Forest Biomass}

Assessing the potential for reducing greenhouse gas emissions through strategic use of biomass resources requires a method to account for biomass resources for two reasons. First, forest resources are tracked to measure the availability of forest products for their traditional uses, such as pulp, paper, energy, and other wood products. Second, forest resources are tracked to measure gross and net carbon uptake and releases. Biomass absorbs carbon dioxide through growth and releases it through respiration and decay, which results from natural causes and may be increased by human activities (e.g., logging residues). This section describes the methods and assumptions used to account for biomass resources in the CONEG region forests, and provides a brief overview of the region's land use patterns and forest characteristics, ownership patterns and land conversion trends.

\subsection{Definitions and General Procedures}

This report considers forest biomass on lands classified by the U.S. Forest Service as "forest lands". Forest lands are lands that are at least 10 percent stocked with trees of any size, or were formerly stocked at this level and not currently slated for development. The minimum acreage for each parcel is one acre. Besides forest land, there are four other land types considered: cropland, pasture, and other land (including urban areas).

Other vegetative matter that sequesters carbon, such as crops or shrubs, are not considered in this report. Crops are not considered because they are harvested regularly and thus do not constitute long-term carbon sinks. Shrubbery should be considered but no inventories exist on shrub volume or carbon uptake.

The largest subset of forest land, and the one considered by this report as land available for biomass harvesting is timberland. Timberland is forest land which is capable of producing at least 20 cubic feet per acre per year (about 0.67 green ton/yr) of industrial wood.

Finally, forest lands were further divided into the two major forest types: hardwood and softwood forests. Each forest type is a forest where a plurality of the trees are in one of these categories. In certain forest types, the plurality may be much larger than in other types. For example, a pine forest tends to be predominantly softwood trees whereas an oak forest tends to be very mixed.

Biomass is the quantity of organic material present in a tree above ground. It is measured in weight on a freshweight basis, i.e. wet (or green) wood. Some definitions used here are: 
- Growing stock trees are trees of commercial species, not including cull trees.

- $\quad$ Cull trees are trees which are rough or rotten and hence potentially marketable for fuelwood but not for wood products.

- Growing stock volume is the volume of growing stock trees 5.0 d.b.h. (diameter at breast beight) and larger from a 1 foot stump height to a minimum 4.0 inch top or to the point where the central stems breaks into limbs.

The data set used in this analysis were the series Forest Statistics and Biomass Statistics published by the U.S. Forest Service. These statistical publications are prepared decennially with the most recent editions published in 1986. The Biomass series is newer than the Forest series and was not available for all states. When a biomass series was not available for a particular state, the Forest Statistics were used and extrapolated to estimate biomass based on the relationships between biomass and growing stocks in other states.

These series provided basic information on stocks, yields (net annual growth), acreage, and the output of timber products. They also provided, either directly or indirectly, the conversion factors used at several points in this analysis.

\subsection{Baseline Forest Data}

The baseline forest data is the data from which the Current Policy scenario is developed. It assumes no change in growth rates, harvesting practices, or other forest characteristics from the current situation. In addition to the data described below, the baseline includes certain levels of planting and thinning which are described in Section 5.3 .

\section{Forest Acreage}

The acreage of forest lands was assigned to hardwood and softwood species based the plurality of acreage of that type. As noted above, the use of plurality of forest stocking to characterize the hardwood and softwood forest types introduces a certain degree of error into the analysis.

\section{Biomass Stocks}

The total stocking of all live material, including cull trees, was used to compute the total mass of softwood and hardwood biomass in each state. This total biomass 
from trees in the forests was divided by total forest acreage to provide a tons/acre stocking figure. Biomass stocking per acre were computed from either Forest Statistics or Biomass Statistics for all states except New York and Pennsylvania. For these two states, whose Statistics were published over ten years ago, the stocking was increased linearly from the year of the data to the present. The increase was based on the predicted growth rate as developed below.

\section{Biomass Yields}

The key element in this analysis is yield, or the annual volume increase in biomass stock. Yields are poorly understood as they are function of many variables. Robert Seymour, in the conclusion of the Timber Supply Projections for Maine, 19802080, writes:

Further refinements must come from better information on the key forest dynamics, particularly in better documenting actual harvest levels and quantifying forest yields (Seymour, 1989).

Seymour's analysis of Maine's forest resources considers yield as a function of four key variables: soil type, tree species, management class and age. This results in over 215 forest types (Seymour, 1989). Owing to the constraints of this project, a simpler method was used to estimate yields.

The yields used in this study are computed from the annual increase in trees classified as growing stock, including cull trees that are not dead or rotten. ${ }^{27}$ The annual yield figures presented below are net of annual deaths, and are thus below the gross annual yield assumptions used in our model.

The model used in our analysis (LEAP) predicts forest yield as exponential. That is, if a forest were to grow 2 percent annually with no cutting, by the end of 20 years there would be 1.48 times as much wood as in the base year. Yields are thus expressed as a percentage annual growth. The data obtained from the Forest Service is the annual average volume increase over a ten year period. For simplicity this figure was used to establish an annual rate of growth by dividing by the final year's stock. Thus, the estimate likely underestimates the actual rate of increase. Seymour noted this but said that it is "probably the best approach" (Seymour, 1991). This view is echoed by the Forest Service which writes:

"The assumptions used to develop the volume projections were considered realistic but conservative. Growth per acre of commercial forest land is not assumed to increase even though New York's forest are growing at 36 cubic feet

\footnotetext{
${ }^{27}$ The annual increase is the volume increase in the portion of the tree, for trees larger than 5 inches d.b.h., from a 1 foot stump to a minimum 4 inch top.
} 
per acre per year but are capable of growing almost twice as much." (Considine, 1984).

Two data sets provide a good estimate of yields. Earlier Tellus Institute work provides a baseline from which to compare the estimates used in the CONEG region study. In an earlier Tellus report yields are estimated as follows:

Our low-yield estimate for biomass yield was taken from the rule of thumb that an acre of unmanaged forest land can be expected to yield "one fourth to onethird of a cord ( 80 c.f., 2.5 tons) of growing stock" annually (Tellus, 1987).

This amounts to about 0.62 tons per acre or 20 to 26 cubic feet for growing stock. Seymour's paper provides another set of estimates reproduced in Table 4.1 below. These estimates are in terms of merchantable cubic feet (including rough cull, non-growing stock trees).

Table 4.1

Mean Annual Growth

cubic H/acre/year (tons/acre/year)

\begin{tabular}{llllll}
\hline Site Class & \multicolumn{2}{c}{ Empirical } & \multicolumn{3}{c}{ High Yield } \\
Good & 36 & $(1.12)$ & 89 & $(2.78)$ \\
Fair & 24 & $(.75)$ & 77 & $(2.41)$ \\
Poor & 20 & $(.625)$ & NA & (NA) \\
\hline
\end{tabular}

The biomass yields used in this study are computed from growing stock and scaled to total biomass (to account for its greater volume). In general, biomass in the forests of the region has a stocking of about 100 green tons per acre. Of this, about 40 50 tons per acre is growing stock. Using the CONEG state average growth rate of 2.23 percent for hardwood trees and 1.61 percent for softwood trees the yields shown in Table 4.2 can be computed:

Table 4.2

Estimated Per Acre Yields for Growing Stock

Hardwood 1.00 tons/acre or $32 \mathrm{cf} /$ acre $(@ 2.5 \mathrm{t} / 80 \mathrm{ct})$

Sottwood $\quad .72$ tons/acre or 27 cf/acre (@2.1 t/80cf) 
The total biomass yields are much larger than these estimates because total biomass stocking is $80-100$ tons per acre, rather than $40-50$ tons/acre for growing stock only. We thus obtain net annual yields of about 1.4 to 2.0 tons per acre for the forests in the CONEG states. In our detailed analyses, the stocks and yields are computed in the manner described above for each CONEG state. The net biomass yields used in this study are consistent with those developed by Dr. Harold Young. Dr. Young writes that the data "suggest a modest annual biomass productivity for planning purposes of 3.0 complete fresh tons per acre" (Young, 1982).

\section{Dead Trees}

Dead trees are an important aspect of forest resources and forest ecology. They provide fuelwood, habitat for various animals and other organisms, and contribute to the production of forest soil and other plant life. Their use as an energy resource could reduce net carbon emissions; the release of carbon dioxide at the point of combustion is essentially equal to the release from their decay in the forest, but at the same time they displace fossil fuel combustion and its emissions.

In this study, fifty percent of dead trees were assumed to be available for fuelwood harvest. Several studies identify them as important sources of firewood ${ }^{28}$ so they were included as such in the analysis. Despite this, the importance of dead wood to the forest ecology would require that its removal be limited; to the degree feasible, the carbon cycle should be closed locally. We have not investigated the forest ecology requirements and constraints that might warrant a change in the assumptions made here.

The "production" of dead trees was modelled using the same data sources used to compute forest yields. Forest yields are the annual growth in the forest, net of deaths. The dead tree account is used to distinguish between the gross and net growth of forest biomass in our accounts.

\subsection{Forest Management}

\section{Planting}

Tree planting is not widespread in the forests of the CONEG region. Nonetheless, new forests are being established at an accelerating rate in the CONEG states as older stands are being cut. The stocking of this new forest is not well understood and varies widely across forest type, geography, soil conditions, and other

\footnotetext{
${ }^{23}$ Considine noted that "the majority of fuelwood is reported to have come from salvable (sic) dead trees." page 48
} 
factors. For simplicity, in this study we have assumed that all 20 year old forests established in the region have a stocking of 30 tons/acre, which is the average biomass of seedlings and saplings for forests in Vermont, New Hampshire, and Maine. This stocking is used when a forest is planted during the study period. Growth in this study is linear over the study period for planted forests. Thus a ne ' forest will have a stocking of 0 , in 10 years a stock of 15 tons/acre, and in 20 years a stocking of 20 tons/acre.

\section{Thinning}

Thinning is a forest practice that increases the yields of established or newly established forests. A wide variety of thinning regimes exist, ranging from a single thinning to more frequent thinning. For most forest owners thinning is not considered an economical activity (USDA, 1990a) but some limited thinning does exist in the CONEG states. This study has used statistics on acreage which is considered "improved" by the U.S. Forest Service as a proxy for thinning activities (USDA, 1990b).

We have assumed that thinning achieves a doubling of annual yields from current levels in the CONEG states' forests. This yield was frequently mentioned as a ballpark figure for the increased yield. It is below the yield noted by Seymour which ranges from 3.4 times (planting + herbicides) to 6.4 times (genetics) (Seymour, 1989). We have lowered the 3.4 times yield to 2 times to conform with our conversations and to account for the infrequent use of herbicides. The actual levels of current planting and thinning ("improved acres" in Forest Service terminology) activities are presented in th Table 4.3 below. To simplify the computations, this study has included all planting ac ities when they exceed 2000/acres/year per state. 
Table 4.3

Current Forest Management Practice on

All Forest Land

FY 1989

\begin{tabular}{lc} 
& Acres/Year \\
Planted & Improved \\
Acres & Acres \\
\hline
\end{tabular}

Connecticut

Delaware

Maine

Massachussetts

New Hampshire

New Jersey

New York

Pennsylvania

Rhode Island

Vermont

Total
929

824

10,225

1

281

572

6,338

1,509

101

655
322

34

76,805

2,961

4,013

260

2,909

2,242

229

2,157

Source: FY 1989 Forest Planting Report, U.S.F.S., March 1990

\section{Baseline Harvesting and Processing}

The removal of trees from the forest produces losses at two stages: losses from harvesting, and losses from processing at sawmills. Each of these are sources of greenhouse gas emissions if they are left to decay (about one-quarter of the biomass weight is carbon; thus, one green ton of decaying biomass residues will emit 0.25 tons of carbon and about 0.9 tons of $\mathrm{CO}_{2}$. (This release is offset by the same amount of carbon taken up from the atmosphere, if the harvesting is done sustainably.) On the other hand, if the residues are used as fuel they would still emit the carbon dioxide when combusted but would displace emissions from fossil fuel combustion rather than add to those emissions. 


\section{Harvesting Losses}

Three factors influence harvesting losses: geography, harvesting practices, and species. Geography is important because it determines the willingness of the landowner or scavenger to remove residues from the forest. Harvesting practices produce much different residue volumes. Whole tree chipping and harvesting for energy production produces small amounts of harvesting residues whereas selective harvesting for commercial lumber may produce much higher residue volumes. Finally, the species influences the residue volume. Hardwoods have more tree mass in the tops that do softwoods, and within species there are differences as well. These three factors make it difficult to develop consistent loss estimates for the CONEG region.

Losses from harvesting were estimated using estimates developed from the harvesting of growing stock. Growing stock is the stem portion of the tree whereas biomass includes both the stump and the leaves and branches. These two components equal about 25 percent (USDA, NC 268) of the above-ground biomass. Numerous estimates exist of the losses from harvesting operations.

Thomas Considine estimates that 19 percent of growing stock harvests remain in the forest as logging residues. However he notes that:

Any utilization of growing stock residue, such as upper stems or trees knocked down, that takes place after the survey crews leave is not tallied. Yet some activities, such as fuelwood cutting, often take place after harvesting is completed.... In all likelihood, our estimate of logging residues overestimates what is left in the woods because some portion of it, perhaps a high portion, has been subsequently cut for fuelwood or other products. (Considine, 1984).

Considine's estimate was developed in 1980. Since 1980 there have been dramatic changes in the way that timber is harvested. Figure 4.1 provides estimates for several CONEG states of the amounts of logging residue produced. 
Figure 4.1

Recent Forest Management Practice

on All Forested Land in the CONEG States

\begin{tabular}{|lcc|}
\hline State & $\begin{array}{l}\text { Year of } \\
\text { Data }\end{array}$ & $\begin{array}{l}\text { \% of Harvest } \\
\text { as Logging } \\
\text { Residue }\end{array}$ \\
Pennsylvania & 1976 & $20.18 \%$ \\
New York & 1980 & $18.87 \%$ \\
Vermont & 1982 & $8.02 \%$ \\
New Hampshire & 1982 & $17.61 \%$ \\
Maine & 1982 & $7.82 \%$ \\
Mass. & 1984 & $6.82 \%$ \\
Rhode Island & 1984 & $4.24 \%$ \\
Delaware & 1985 & $9.46 \%$ \\
Connecticut & 1985 & $8.52 \%$ \\
Maryland & 1986 & $7.51 \%$ \\
New Jersey & 1987 & $2.53 \%$ \\
& & \\
Source: Forest Statistics & & \\
\hline
\end{tabular}

The residue levels appear to have fallen significantly, especially for softwood. The current average residue loss from Figure 4.1 is in the 7 percent range. This study has used this 7 percent as a percent of the growing stock volume (75 percent). This produces a loss on biomass of 5.2 percent to forest residues from logging.

An alternative estimate of logging residues is provided by the Office ' $f$ Technology Assessment. They note that in the forest of the Northern Unit:d States ${ }^{*}$ 16 thousand dry tons of residue was produced from a harvest of 32 thousand, or about 50 percent additional residue (OTA, no date). This estimate was computed in the early 1970's so it might not have much relevance today. Like Considine they note numerous difficulties in estimating residues. They write:

The regions are not defined 
There is some uncertainty as to whether various logging residues studies are in agreement as to what constitutes non-growing stock logging residue. (OTA, no date).

Some of the uncertainty concerning residues arises from how residues are created. When a tree is removed, different removal processes create different volumes of residue. Removal of the tree for energy or pulp generally involves removal and chipping of the entire tree. Thus, about half of the biomass in the growing stock residues and tops are taken and used, and stumps generally left behind. Harvesting of trees for commercial wood - for milling into wood products - involves removing just the growing stock with the rest of the biomass left in the forest. However, we assume here that onehalf of the residues and tops that are otherwise left behind are taken from the forest for fuelwood use. These two processes are not exclusive as some commercial logging may be accompanied by chipping operations. ${ }^{30}$

Table 4.4 provides the estimates of the forest losses and residue use for firewood and paper and pulp wood collection, as percentages of green tons throughput. About 30 percent of the total biomass volume is left behind as tops, stumps and other residues, and 42 percent of these residues are assumed to be used as fuels. In our biomass policy scenarios we assume that a greater fraction of the residues (67 percent) are collected for use as wood to fuel increased fuelwood demands, primarily in the electric sector.

Table 4.4

Firewood and Commercial Wood Harvesting Accounts/Losses Assumptions

\section{LOSS ASSUMPTIONS}

In the Forest

\begin{tabular}{|c|c|c|c|}
\hline & & In the $F$ & \\
\hline & $\begin{array}{c}\text { Original } \\
\text { Tree }\end{array}$ & $\begin{array}{l}\text { Left in } \\
\text { Forest }\end{array}$ & $\begin{array}{l}\text { Residues } \\
\text { Used }\end{array}$ \\
\hline Growing Stock & 69.8 & & \\
\hline Growing Stock Residue & 5.2 & 2.6 & 2.6 \\
\hline Stump & 5.0 & 5.0 & \\
\hline Tops & 20.0 & 10.0 & 10.0 \\
\hline Total & 100.0 & 17.6 & 12.6 \\
\hline
\end{tabular}

${ }^{30}$ A further complication to estimating losses involves trees that are destroyed during lugging. Dead trees provide considerable fuelwood and may be considered part of the logging residues noted above. 


\section{Processing Losses}

Logging residues from processing result from the sawing and debarking of trees. These residues can be quite significant yet most are used in the sawmill. For the southern New England region, the Forest Service writes:

The volume and proportion of unused residue from the region's sawmills have dropped substantially in recent years. In 1971, 1.7 million cubic feet, or one-fifth of the wood residue, went unused. In 1984, about one-fourth as much - less than 440,000 cubic feet - went unused (USDA, 1987).

The Forest Service estimates that 97 percent of the residues that are produced are "used". Useful uses include: Agriculture (49 percent), Fuel (34 percent), Fiber (11 percent), Other ( 3 percent). The total amount of residues which decay and produce greenhouse gases is then:

$$
\text { agriculture }+ \text { unused }=52 \text { percent of residues produced. }
$$

The volume of residues that are produced is dependent on the milling technology used and other factors. For this study, we estimate the 1988 residue level from the 1984 residues in southern New England, about 35 percent of the total volume processed. ${ }^{31}$

Table 4.5 on the following page provides the breakdown. About 30.2 percent of the original biomass volume is uncollected as raw material for commercial wood products (and 42 percent of this is collected in the forest for use as fuel). Then, at the sawmill about 35 percent of the material processed (or 25.3 percent of the original biomass) is lost in the process, while about half of these loses (12.2 percent of the original biomass) is used for firewood. The final wood product from harvesting and processing commercial wood thus embodies 47.1 of the original biomass.

${ }^{31}$ Table 1 and Table 7, Timber Industries of S.N.E. The estimate for 1988 does not coninrm with the expected trend. It would be estimated that residues would decline over time as milling equipnient impioved. However, the increase in residues may be a function of the use of smaller trees which produce more residues. 


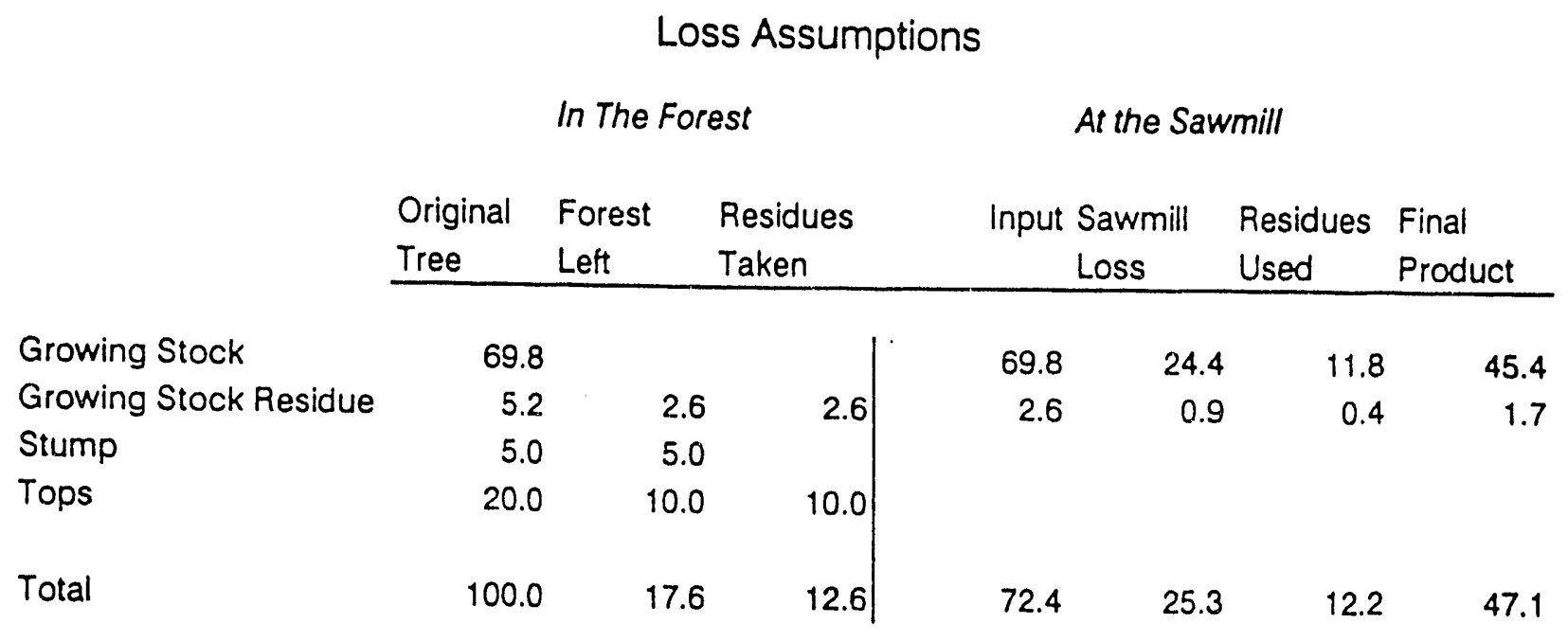

\subsection{Forest and Other Land Use Patterns}

The forests of the Northeastern United States contribute to the region's energy systems, to its commercial products, and to its greenhouse gas balances: they also provide recreational opportunities, settings for human settlement, habitats for wildlife, water supply and watershed protection, and soil production and protection. Because biomass plays multiple roles in the region's economy, ecology and public amenity, several characteristics of the region's forests interact to dictate how this resource can be utilized for energy supply and greenhouse gas mitigation strategies.

Table 4.6 provides land use accounts for each state in the CONEG region, broken out into hardwood forest, softwood forest, cropland, pasture land, protected forest, and other land (urban and other built environments, water bodies, etc.). 
Table 4.6

CONEG States Land Areas, 1000 Acres

\begin{tabular}{|c|c|c|c|c|c|c|c|}
\hline & $\begin{array}{l}\text { Hardw } \\
\text { Forest }\end{array}$ & $\begin{array}{l}\text { Softw } \\
\text { Forest }\end{array}$ & Crop & Pasture & $\begin{array}{l}\text { Prot } \\
\text { For }\end{array}$ & Other & Total \\
\hline Connecticut & 1,530 & 247 & 182 & 42 & 48 & 1,067 & 3,116 \\
\hline Maine & 7,051 & 10,009 & 591 & 138 & 547 & 1,500 & 19,836 \\
\hline Mass & 1,747 & 1,181 & 214 & 52 & 296 & 1,517 & 5,007 \\
\hline New Hamp & 2,640 & 2,175 & 124 & 51 & 175 & 593 & 5,758 \\
\hline Rhode Island & 312 & 59 & 24 & 5 & 33 & 242 & 675 \\
\hline Vermont & 3,144 & 1,278 & 566 & 320 & 122 & 503 & 5,933 \\
\hline New England & 16,424 & 14,949 & 1,701 & 608 & 1,221 & 5,422 & 40,325 \\
\hline Delaware & 235 & 141 & 509 & 92 & 13 & 247 & 1,237 \\
\hline Maryland & 1,792 & 632 & 1,602 & 567 & 279 & 1,425 & 6,297 \\
\hline New Jersey & 1,196 & 669 & 605 & 97 & 142 & 2,070 & 4,779 \\
\hline Pennsyivania & 14,995 & 929 & 4,779 & 1,588 & 902 & 5,586 & 28,779 \\
\hline Mid-Atlantic & 18,218 & 2,371 & 7,495 & 2,344 & 1,336 & 9,328 & 41,092 \\
\hline New York & 12,905 & 2,501 & 4,327 & 1,917 & 3,100 & 5,464 & 30,214 \\
\hline CONEG & 47,547 & 19,821 & 13,523 & 4,869 & 5,657 & 20,214 & 111,631 \\
\hline
\end{tabular}

Source: U.S. Forest Service

From Table 4.6 it can be seen that about 65 percent of the total land area in the CONEG states is forested and 8 percent of this is protected in public forests. New England has the highest forested percentage at 80 percent, while New York has the highest fraction under protection at 20 percent. Putting greater amounts of forest land under protection would help meet certain environmental aims, while constraining the use of forest biomass resources for energy and other products. The amounts of non-forested arable land (defined here as crop and pasture land) is about 16 percent of total land area in the CONEG states, highest in the Mid-Atlantic states at 24 percent, and only 6 percent in the New England states. To the degree that an increase in forested land - for fiber, energy, or $\mathrm{CO}_{2}$ sequestration - will require the use of currently unforested productive land, the greatest opportunities exist in the New York and Mid-Atlantic regions (primarily in New York, Pennsylvania, Maryland). This would compete, however, with other important uses, especially food production. These further breakdowns are summarized in Table 4.7. 
Table 4.7

Percent Shares of Key Land Types

in the CONEG States

Forest Land

$\%$ of Total
$\%$ of Forest

Protected
$\%$ of Forest Crop/Past

in Hardwood \% of Total
New England

Mid-Atlantic

New York
80

53

61

65
4

6

20

8
52

88

84

71
6

24

20

16

\subsection{Overview of the Forests}

The forests of the Northeastern states face an uncertain future. In close proximity to the densest population centers in the United States, the forests provide recreation and products for urban residents, yet face increasing stress from these same urbanites. At the same time, they provide benefits to and suffer the impacts of rural settlement and industrial activities. These forests also represent a natural endowment of intrinsic value in the variety of flora and fauna that they support. They provide important sources of clean water, rare organisms, and other natural values. Increasing urbanization, increased wood use, forest development and conversion all threaten the forests as do other threats such as global warming, acid rain, disease and pollution generally.

As can be seen in Table 4.6, the forests of the CONEG states cover nearly 70 million acres. Hardwood forest land predominates ${ }^{32}$; over 70 percent of the unprotected forest is hardwood, while softwood accounts for less than 30 percent. This is not uniform throughout the region, as hardwood in the New York and the Mid-Atlantic regions are 84 and 88 percent, while in New England is only 52 percent. Maine is the only state in which land dominated by softwood species predominates, at 58 percent. Typically, paper and pulp and wood products are provided by softwood while energy resources come from the hardwoods.

During the past few decades, these forests have grown in size as agricultural land has been abandoned and forests have expanded. The growth of the forest is a welcome development after decades of deforestation. The future, however, may not be so bright as the forest faces new threats from the development of vacation properties.

\footnotetext{
${ }^{32}$ Land is designated as hardwood or softwood according to the U.S. Forest Service characterization in terms of the majority species.
} 
Deforestation in the Northeast has occurred in several waves. The census of 1880, warned: "The forests of New York are no longer important as a source of general lumber supply ... White oak .. has become scarce ... elm, ash, hickory, and other woods are reported scarce." The situation was echoed in Pennsylvania where "merchantable pine has now almost disappeared ... manufacturers using hardwood report great deterioration and scarcity of material." (Perlin, 1989). Later waves of deforestation in the Northeast occurred with the railroad, and within the past three decades new road construction has put much more of the forest within reach of new harvesting technologies perhaps foreboding another period of deforestation (USDA-FS, April 1990).

The growth of the forests of the region has generally not been planned or managed. Forest management in the Northeastern states is generally limited when compared to the intensive management of the Southeast or Northwest United States, owing primarily to its different landholding patterns and the predominance of hardwoods in the Northeast. Planting is generally by natural means, and thinning, although becoming more common, is still the exception rather than the rule.

Because of the lack of management and past timber harvesting practices such as "high grading"33, much of the high quality wood has been removed from the forests of the region. Many foresters contacted during this study commented that harvesting the forests of the CONEG states would benefit these forests by releasing overstocked stands and replacing poor quality trees with "higher quality" trees. Replacement of the existing forest with a new forest could, on the other hand, be detrimental to wildlife and recreational activities.

\section{Ownership Patterns}

The forest ownership pattern in the CONEG states is unique in the United States. The forests of the Northeast are overwhelmingly owned by small private landowners. Over 72 percent of the forest land is held by private non-industrial landowners, and in small parcels. The Northwest, by contrast, has much larger public holdings in the National Forests. Table 4.8 below presents the land ownership patterns of the CONEG states. The total commercial forest land in Table 4.8 corresponds to the total of hardwood and softwood forest land area given in Table 4.6. Protected forest land, given in Table 4.6, is excluded from Table 4.8 as it is not available for commercial exploitation.

${ }^{33}$ High grading removes quality trees and leaves poor quality ones. 
Table 4.8

Commercial Forest Ownership Breakdown

Thousands of Acres

\begin{tabular}{|c|c|c|c|c|c|}
\hline & $\begin{array}{l}\text { National } \\
\text { Forest }\end{array}$ & $\begin{array}{l}\text { Other } \\
\text { Public }\end{array}$ & $\begin{array}{l}\text { Forest } \\
\text { Industry }\end{array}$ & $\begin{array}{l}\text { Other } \\
\text { Private }\end{array}$ & Total \\
\hline CT & - & 216 & 3 & 1,556 & 1,7 \\
\hline ME & 45 & 644 & 8,016 & 8,353 & 17,600 \\
\hline MA & - & 430 & 66 & 2,432 & 2,929 \\
\hline $\mathrm{NH}$ & 471 & 196 & 657 & 3,486 & 4,812 \\
\hline $\mathrm{RI}$ & - & 45 & 4 & 322 & 371 \\
\hline$V T$ & 183 & 246 & 409 & 3,583 & 4,422 \\
\hline DE & $\cdot$ & 13 & 31 & 331 & 376 \\
\hline MD & - & 245 & 130 & 2,047 & 2,424 \\
\hline NJ & - & 464 & 0 & 1,400 & 1,864 \\
\hline PA & 49 & 2,987 & 964 & 11,488 & 15,489 \\
\hline NY & - & 979 & 1,034 & 13,392 & 15,405 \\
\hline CONEG & 748 & 6,465 & 11,314 & 48,390 & 66,929 \\
\hline
\end{tabular}

Ownership patterns affect how the forest is used. Commercial land, over 11 million acres, is generally managed for wood production, while small private landowners have wide ranging objectives. Some invest for speculation, some for recreation, some for wood, and others for still different reasons.

The mix between public and private, and between large and small private landowners varies significantly across the CONEG states, as evidenced in Table 4.8. In some areas, such as Northern Maine, large private owners control the vast majority of the land. In other areas, such as in the Adirondacks, public ownership predominates.

Small landowners are a key element in the future of the CONEG forests. Land management is not a full time occupations for these owners, and they have widely differing objectives for the use of their land. These landowners can play a critical and pivotal role by providing timber, preserving land values, contributing to watershed and soil preservation, and protecting forest products and biota.

Small landowners face the most severe pressures to convert their land to other uses. Many of the holdings of small landowners are close to urban areas or transportation corridors making them vulnerable to conversion to other, potentially more 
profitable, uses. Future changes in small landownership represents one of the major unknowns about timber inventories and supplies in the CONEG states.

\section{Land Conversion}

A second major factor influencing biomass supply is land conversion. Although conversions from forest land to a variety of uses have slowed during the recent recession, the patterns established during the 1980's would threaten the Northeastern forests if continued into the next century. The Northern Lands Study warns of the continuation of past trends:

If the forces driving change are allowed to run their course unchecked, it is likely that several generations from now the Northern Forest will not have the attractive landscape, diversity of wildlife, and wealth of public recreation opportunities of today... Although these trends are affected by economic cycles, they have never been reversed. (USDA-FS, April 1990.)

The implications of land conversion are manifold. If the land is cleared when it is converted, forest acreage and wildlife may be adversely affected. Conversion of riparian habitat to residential vacation properties often threatens water quality and habitat. Road building brings people into closer contact with wildlife and is especially threatening to those species that need large areas. Land conversions could negatively affect the supply and quality of the accessible biomass resources of the region.

Finally, land conversions could contribute additional releases of carbon dioxide if the biomass is not sequestered in very long lived products but, rather, decays or is burned.

\subsection{Forest Products Usage}

Table 4.9 below, summarizes the forest products usage in the CONEG states. The energy figures are based on the Public Citizen study, Power of the States, and the forest products figures are based on U.S. Forest Service statistics. 
Table 4.9

Estimated Wood End-Use

in the CONEG States

Trillion Btuth

\begin{tabular}{|c|c|c|c|c|}
\hline & $\begin{array}{l}\text { Paper \& } \\
\text { Pulp }\end{array}$ & $\begin{array}{l}\text { Wood } \\
\text { Products }\end{array}$ & $\begin{array}{l}\text { Res/Comm/Ind } \\
\text { Fuel Wood }\end{array}$ & $\begin{array}{l}\text { Wood for } \\
\text { Electric Power }\end{array}$ \\
\hline Connecticut & 0.1 & 2.2 & 14.0 & $\ldots$ \\
\hline Maine & 61.8 & 25.7 & 30.6 & 51.0 \\
\hline Mass & 0.6 & 4.0 & 27.1 & $\cdots$ \\
\hline New Hamp & 10.0 & 8.0 & 20.8 & 11.0 \\
\hline Rhode Is & $\ldots$ & 0.2 & 4.6 & $\ldots$ \\
\hline Vermont & 6.9 & 5.6 & 19.9 & 5.0 \\
\hline New Eng & 79.4 & 45.7 & 117.0 & 67.0 \\
\hline Delaware & 0.3 & 0.7 & 4.1 &.-- \\
\hline Maryland & 0.1 & 5.5 & 21.4 & 0.5 \\
\hline New Jersey & $\cdots$ & 0.6 & 17.5 & $\ldots$ \\
\hline Pennsylvania & 18.8 & 22.8 & 69.1 & 6.9 \\
\hline Mid-Atlantic & 19.2 & 29.6 & 112.1 & 7.4 \\
\hline New York & 17.3 & 18.4 & 75.5 & 1.5 \\
\hline CONEG & 115.9 & 93.7 & 304.6 & 75.9 \\
\hline
\end{tabular}

As can be seen from Table 4.9, in absolute terms, New England has the largest production of wood and paper and pulp products (60 percent of the CONEG region total), the largest consumption of end-use wood fuels (38 percent of the CONEG region total), and the greatest amount of wood-electric power supply (88 percent of the CONEG region total). In relative terms, wood used for end-use and electric power production is about 7.5 percent of total end-use energy requirements in New England, 2.1 percent in the Mid-Atlantic states, and 2.8 percent in New York. ${ }^{35}$ End-use wood energy per capita is about 9.5 MMBtu for New England, 4.7 MMBtu for Mid-Atlantic, and 4.3 MMBtu for New York. Adding wood for electricity production increases the New England consumption to about 15 MMBtu per capita, and the Mid-Atlantic figure to about 5 MMBtu per capita.

\footnotetext{
${ }^{34}$ We assume 13.7 Million Btu per ton of dry wood.

${ }^{35}$ Here, we account wood used in electricity production, rather than the end-use electricity produced (reduced by a factor of 0.2 to reflect a heat rate of $16.5 \mathrm{MMBtu} / \mathrm{MWh}$ ).
} 
More detailed state-specific information on land use, forest biomass, and wood uses is provided in Appendix B. More detailed summary descriptions of the forests and commercial forest activities of each state can be found in Appendix C. This background information, based in part on information provided above in Tables 4.6 through 4.9 , is useful for understanding the opportunities and constraints relevant to the biomass related energy supply and greenhouse gas mitigation strategies developed in Section 6. 


\section{Current Biomass Policy: Reference and Conservation Cases}

In Section 3 we developed a Reference Case, which projected carbon and methane emissions for the CONEG region, based on continuation of current trends in energy demand and supply - including the demand for and supply of biomass fuel resources - over the next thirty years. We also described an Energy Conservation Case, which assumes that energy demand will be managed more intensively over that period, thus reducing the need for traditional energy resources, including biomass fuels. In this section, we round out the Reference and Conservation case results by accounting for the non-combustion part of the biomass carbon cycle: carbon uptake from tree growth and carbon releases from the decay of forest residues.

Two of our four scenarios result:

1) Current Biomass Policy - Reference Case

2) Current Biomass Policy - Conservation Case

Current Biomass Policy starts from the existing forest biomass resource and assumes no significant changes in forest management practices or the growth of wood energy or other wood product demands. In the next section, we will maintain the Conservation Case energy demand assumptions and introduce two additional biomass scenarios, each assuming a successively greater contribution of biomass to the region's overall energy mix.

\subsection{Current Biomass Policy - Reference Case}

The Reference Case embodies the assumptions about future energy consumption and biomass product demand which were described in Section 3. This section discusses the impacts of Current Biomass Policy on forest biomass resources and on the forest carbon cycle and resulting emissions, given the Reference Case energy assumptions.

\section{Wood Energy/Product Demand}

Current and future demand was considered for the three major wood products: fuelwood, pulpwood, and construction wood. We estimated the current and future demand for these resources by five sectors - residential fuelwood, commercial/industrial fuelwood, paper and pulp industry wood requirements, fuelwood for electricity production, wood for commercial products (sawmills). Some demands spanned more than one sector. Fuelwood, for example, is used both by the residential sector and by the electric generating sector. 
The current demands for each material and for fuelwood for electric generation are given in Table 5.1. This table states all demands on forest resources in common units of green tons in the forest. (In Table 3.2, we presented the end-use demands in energy terms.) We assumed 13.8 million Btu per dry ton of wood and 46 percent water content by weight in fresh or green forest biomass. End-use demands, such as dry tons and fuelwood consumption in Btu were converted to green tons using the loss factors presented in Section 4.

Residential and commercial/industrial firewood demand is assumed to grow at the same overall rate as energy demand generally. Pulpwood demand is assumed to grow slowly over the forecast period, based on projections prepared by RISI for the United States and Maine. For states other than Maine, we have used the U.S. forecast increase of 1.33 percent per year, whereas for Maine we have used the growth rate of 1.19 percent per year. We have also included changes in the relative shares of hardwood and softwood in providing the feedstock for pulp. According to RISI, hardwood is increasing in use as a paper coating thus increasing demand on hardwood stocks.

Construction wood demand has been forecast based on information for the United States as well as specific forecasts for Maine. The United States forecast, based on the U.S. Industrial Outlook, forecasts a 1.5 percent annual increase in construction wood demand. Conversely, the Forests for the Future report provides a much different forecast for Maine of negative 3.67 percent annually. We have accepted this forecast as reflective of specific conditions in Maine. 
Table 5.1

Biomass Demand Inputs

1988 Base Year

(million green tons)

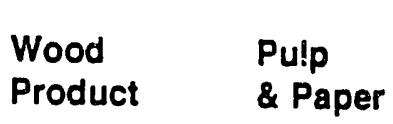

Resid. \&

Comm. Electric Total

Fuelwood Fuelwood

NorthEast

$\begin{array}{lrrrrr}\text { Connecticut } & 1 & 0 & 3 & 0 & 4 \\ \text { Maine } & 9 & 12 & 6 & 10 & 37 \\ \text { Massachusetts } & 1 & 0 & 5 & 0 & 6 \\ \text { New Hampshire } & 3 & 2 & 4 & 2 & 11 \\ \text { Rhode Island } & 0 & 0 & 1 & 0 & 1 \\ \text { Vermont } & \frac{2}{16} & \frac{1}{15} & \frac{4}{23} & \frac{1}{13} & \frac{8}{67}\end{array}$

Mid-Atlantic

Delaware
Maryland
New Jersey
Pennsylvania

0
2
0
8
10

$\begin{array}{rr}0 & 1 \\ 0 & 4 \\ 0 & 3 \\ 4 & \frac{13}{21}\end{array}$

$\begin{array}{lr}0 & 1 \\ 0 & 6 \\ 0 & 3 \\ 1 & \frac{26}{36}\end{array}$

New York

New York

6

3

14

Note: Converted from dry tons to wet tons with a water loss of 54 percent and collection losses of 17.8 percent for all wood types. These results will differ from LEAP results as they do not include the offsetting uses of milling residues.

Wood for electric generation, using conventional wood-electric power plants, is assumed to increase approximately two-fold from $806 \mathrm{MW}$ to about $1676 \mathrm{MW}$. The overall increase was assumed to be one-third of the $2600 \mathrm{MW}$ embodied in the DOE/EIA 1990 Annual Energy Outlook projections. These additions are about one-third those assumed in the most recent DOE projections. The reduction was intended to reflect more modest biomass energy policy goals, given current conditions including the relatively low price of oil in the region. Table 5.2 summarizes the wood-electric power supply assumptions for this case. 
Table 5.2

Current Policy Wood-Electric Power Supply

in the CONEG Region

(MW)

\begin{tabular}{lrrccc} 
& 1988 & 1996 & 2004 & 2012 & 2020 \\
New England & 716 & 739 & 763 & 786 & 786 \\
Mid-Atlantic & 77 & 210 & 344 & 477 & 477 \\
New York & 13 & 146 & 280 & 413 & 413 \\
CONEG & 806 & 1095 & 1387 & 1676 & 1676 \\
\hline
\end{tabular}

\section{Results}

The Current Biomass Policy scenario (assuming Reference Case energy demands) provides an indication of what might happen to forest biomass and the forest biomass carbon cycle in the Northeast if basic forest usage and growth patterns do not change.

\section{Forest Biomass Stocks}

Regionally, only New England's forest decline (about 6 percent by 2020), whereas the forests of the Mid-Atlantic and New York grow by about 7 and 18 percent, respectively, over the thirty-year period. Overall, the region's forest biomass is projected to remain stable over this period. Table 5.3 provides the resulis of Current Biomass Policy using the energy projections of the Reference Case.

Table 5.3

Current Biomass Policy

- Reference Case Energy -

Forest Biomass Stocks by CONEG Region (million green tons)

\begin{tabular}{lrrrrr}
\hline & 1988 & 1996 & 2004 & 2012 & 2020 \\
New York & 1,529 & 1,616 & 1,693 & 1,755 & 1,809 \\
Mid-Atlantic & 2,033 & 2,100 & 2,149 & 2,177 & 2,182 \\
New England & 2,732 & 2,700 & 2,666 & 2,623 & 2,560 \\
CONEG States & 6,295 & 6,417 & 6,508 & 6,555 & 6,551 \\
\hline
\end{tabular}


The patterns of modest growth in the Mid-Atlantic and New York regions, and a decline in New England, may signal an end to the growth phase of the past decades. We do not anticipate significant growth in forest resources in the next 30 years. This occurs from the outcome of simple logic: forests only grow so fast and demand continues to increase faster than forests can supply it.

To verify the results of the Reference Case we have consulted Boise Cascade and the author of the Forests for the Future report. According to Sy Balch, Chief Forester of Boise Cascade, the standing inventory in their market region (northern Vermont, NH, Maine, Quebec, and New Brunswick) will decline over the next 30 years. According to Balch, the standing inventory will be replaced with younger and faster growing trees. Mr. Balch saw equal reductions in inventory for hardwood and softwood and slow price increases over time moderating shortages. Price increases are also likely to reduce the competitiveness of biomass projects in the region. We were unable to obtain any quantitative information on Boise Cascade's forecast.

An alternative forecast is prepared by Robert Seymour of the University of Maine. His forecast predicts rapid reductions in forest inventories and shortages in some species over the next 100 years. Especially noticeable are forecast shortages in hardwood stands. Seymour notes that the demand for pulp wood in 1981-1985 was 101 percent of the sustainable level.

Table 5.4 compares the Seymour forecast of standing inventory for softwood and hardwood stocks until 2020. The Reference Case forecast is in general agreement with the Seymour forecast.

Table 5.4

Comparison of Tellus

and R. Seymour Forecasts

of Forest Stocks

Softwood

Seymour

Tellus

Hardwood

Seyriour

Tellus
Maine Inventories

$1990=100$

1990

100

100

100

100
2020

85

62

53

62
Decline

$15 \%$

$38 \%$ 
From our conversations with these two sources, we are confident that the Reference Case forecast is consistent with the current thinking about the forest resources of the Northeast.

\section{Forest Biomass Yields}

In addition to the overall forest stocks, it is important to examine the impact on forest yields, or annual increase to the volume of biomass stock resulting from growth. Forest yields are presented in Table 5.5.

Table 5.5

Current Biomass Policy

- Reference Case Energy -

Forest Biomass Yields by CONEG Region

(million green tons)

\begin{tabular}{lrrrrr}
\hline & 1988 & 1996 & 2004 & 2012 & 2020 \\
New York & 37.12 & 39.18 & 41.05 & 42.66 & 44.14 \\
Mid-Atlantic & 45.28 & 47.16 & 48.81 & 50.24 & 51.18 \\
New England & 63.94 & 64.88 & 65.73 & 66.42 & 66.03 \\
CONEG States & 146.34 & 151.22 & 155.59 & 159.32 & 161.35 \\
& & & & & \\
\hline
\end{tabular}

\section{Carbon Balances}

The gross carbon uptake and releases from forest biomass in the Reference Case are represented in Table 5.6. Carbon uptake results from forest growth, while carbon releases result from the decay of unused dead wood, from residues of firewood, pulp and paper, and commercial wood harvesting activities, and from commercial wood milling residues. The assumptions concerning the losses occurring at each stage in the process were described previously in Section 4.

This table illustrates that although the forests will absorb an increasing amount of carbon over the next 30 years through their growth, increased demands on forest biomass and their associated losses and decay, will offset the gross carbon uptake. On a net basis the carbon uptake in the region's forests increases by about 7 percent over the thirty year 
period. Annual net carbon uptake remains about 9 percent of the carbon currently released annually from energy use in the region.

Table 5.6

Current Biomass Policy

- Reference Case Energy -

Forest Carbon Uptake in the CONEG Region

(million tons)

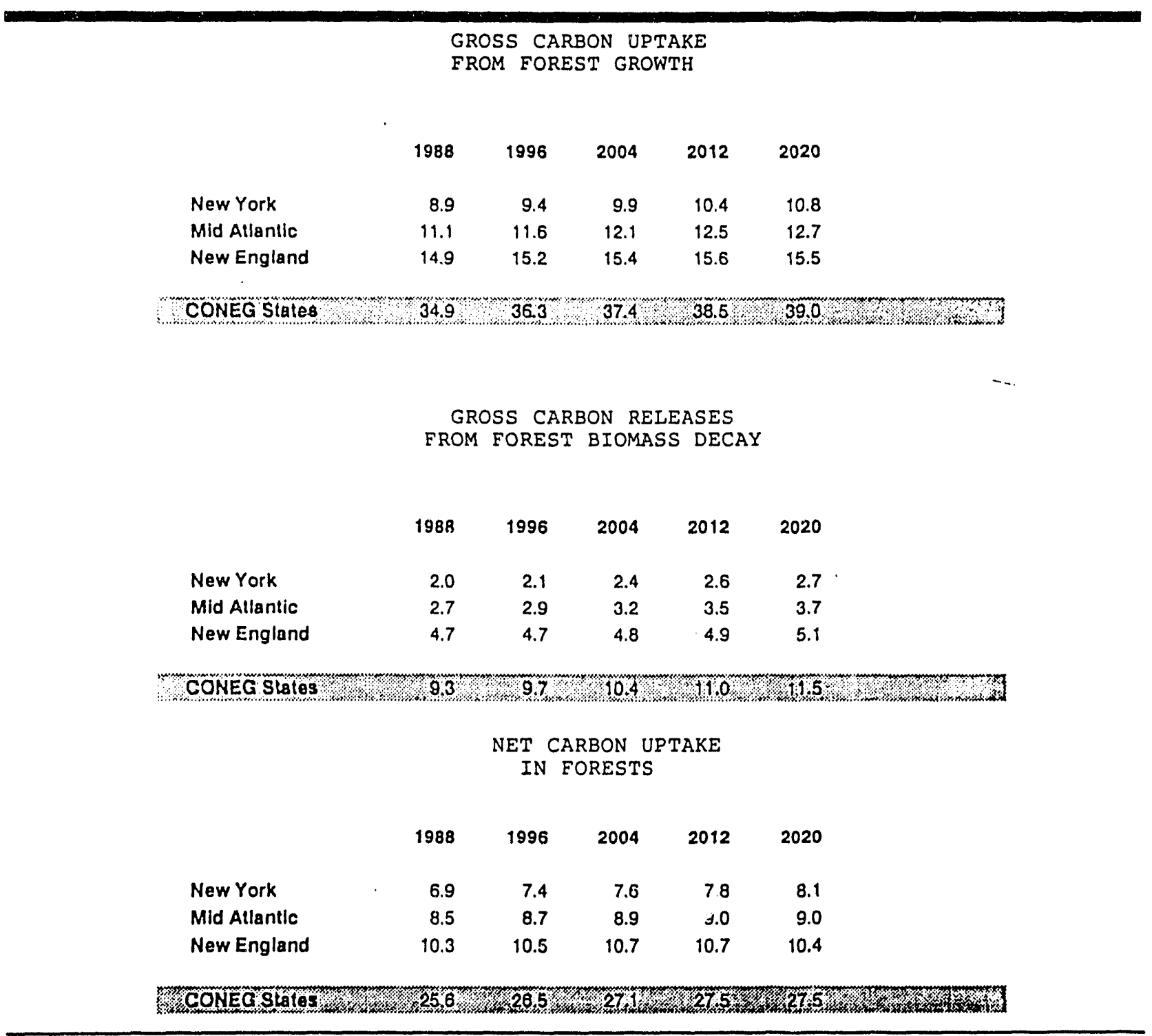


Table 5.7 provides the combined emissions for energy, soliu waste and forests including urban trees) for the Reference Case. Overall carbon equivalent emissions increase by about 25 percent over the 32 -year study period, driven by energy demand growth and continued use of fossil fuels. A decrease of 30 to 50 percent by from current levels by 2020 - arguably required for climate stabilization - would entail a reduction of 45 to 60 percent of projected emissions in that year. Thus, very substantial amounts of conservation and switching from fossil fuels would need to be effected by that time.

Table 5.7

Carbon Balances for the CONEG Region

- Current Biomass Policy -

Reference Case Energy

(Million Tons Carbon Equivalent)

\begin{tabular}{lccccc}
\hline & 1988 & 1996 & 2004 & 2012 & 2020 \\
$\begin{array}{l}\text { Gross Emissions } \\
\begin{array}{l}\text { From Energy } \\
\text { and Solid Waste }\end{array}\end{array}$ & 314.7 & 324.2 & 341.4 & 361.2 & 389.2 \\
$\begin{array}{l}\text { Net Carbon Uptake } \\
\text { From Growing Forest } \\
\text { Biomass }\end{array}$ & 25.6 & 26.5 & 27.1 & 27.5 & 27.5 \\
Net Emissions & 289.1 & 297.7 & 314.3 & 333.7 & 361.7 \\
\hline
\end{tabular}

\subsection{Current Biomass Policy - Conservation Case}

\section{Conservation Case}

The Conservation Case projects future energy and biomass product demand, assuming significant but attainable levels of energy conservation, as described in Section 3. This section will discuss the impacts of Current Biomass Policy on forest biomass resources and on the forest carbon cycle, given the Conservation Case energy assumptions. 


\section{Results}

\section{Forest Biomass Stocks}

Table 5.8 summarizes the impacts of Current Policy on forest biomass stocks in the region. The effect of energy conservation, which is assumed here to reduce end-use biomass demand by more than 20 percent, is to decrease the pressure on forest resources. Thus, by 2020 forest biomass stocks are about 11 percent above 1988 levels and 7 percent above the 2020 levels for the Reference Case energy assumptions.

Table 5.8

Current Biomass Policy

- Conservation Case Energy -

Forest Biomass Stocks by CONEG Region (million green tons)

\begin{tabular}{llllll} 
& 1988 & 1996 & 2004 & 2012 & 2020 \\
New York & 1,529 & 1,621 & 1,715 & 1,811 & 1,914 \\
Mid-Atlantic & 2,033 & 2,108 & 2,185 & 2,267 & 2,351 \\
New England & 2,732 & 2,708 & 2,702 & 2,712 & 2,731 \\
CONEG States & 6,295 & 6,437 & 6,602 & 6,789 & 6,996 \\
\hline
\end{tabular}




\section{Forest Biomass Yields}

Forest biomass yields also increase slightly in the Conservation Case, relative to the Reference Case.

Table 5.9

Current Biomass Policy

- Conservation Case Energy .

Forest Biomass Yields by CONEG Region

(million green tons)

\begin{tabular}{lccccc}
\hline & 1988 & 1996 & 2004 & 2012 & 2020 \\
New York & 37.1 & 39.3 & 41.5 & 43.9 & 46.4 \\
Mid-Atlantic & 45.3 & 47.3 & 49.6 & 52.2 & 54.9 \\
New England & 63.9 & 65.0 & 66.5 & 68.4 & 69.9 \\
CONEG States & 146.3 & 151.6 & 157.6 & 164.5 & 171.2 \\
& & & & & \\
\hline
\end{tabular}

\section{Carbon Balances}

Net annual carbon uptake by the region's forests, shown in Table 5.10, increases from 25.6 million tons in 1988 to 31.4 million tons in 2020, about 4 million tons above the Reference Case. 
Table 5.10

Current Biomass Policy

- Conservation Case Energy -

Forest Carbon Uptake in the CONEG Region

(million tons)

GROSS CARBON UPTAKE

FROM FOREST GROWTH

$\begin{array}{lrrrrr} & 1988 & 1996 & 2004 & 2012 & 2020 \\ \text { New York } & 8.9 & 9.5 & 10.1 & 10.7 & 11.4 \\ \text { Mid Allantlc } & 11.1 & 11.7 & 12.3 & 13.0 & 13.7 \\ \text { New England } & 14.9 & 15.2 & 15.6 & 16.1 & 16.6 \\ & & & & & \\ \text { CONEG States } & & & & \end{array}$

GROSS CARBON RELEASES

FROM FOREST BIOMASS DECAY

$\begin{array}{lllll}1988 & 1996 & 2004 & 2012 & 2020\end{array}$

$\begin{array}{llllll}\text { New York } & 2.0 & 2.1 & 2.3 & 2.4 & 2.5 \\ \text { Mid Allantic } & 2.7 & 2.8 & 3.0 & 3.1 & 3.3 \\ \text { New England } & 4.7 & 4.6 & 4.5 & 4.5 & 4.6\end{array}$

CONEG States

NET CARBON UPTAKE

IN FORESTS

$\begin{array}{lrrrrr} & 1988 & 1996 & 2004 & 2012 & 2020 \\ \text { New York } & 6.9 & 7.4 & 7.8 & 8.3 & 9.0 \\ \text { Mid Atlantic } & 8.5 & 8.9 & 9.3 & 9.9 & 10.5 \\ \text { New England } & 10.3 & 10.6 & 11.1 & 11.6 & 12.0\end{array}$

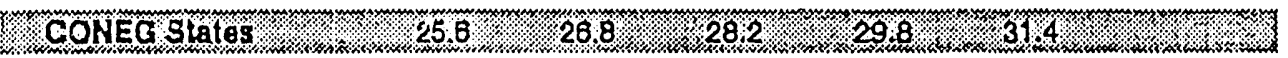


Table 5.11 gives net missions for energy, solid waste and forests for the Conservation Case. The Ccnservation Case achieves a 33 percent reduction in carbon equivalent emissions by the year 2020 relative to the Reference Case, a decrease of about 17 percent from 1988 levels. As noted earlier, this can be realized at essentially no net cost, since the energy Conservation Case assumes energy efficiency gains which can be achieved at costs which are equal to or less than avoided fuel costs.

Table 5.11

Carbon Ealances for the CONEG Region

- Current Blomass Policy -

Conservation Case Energy

(Million Tons Carbon Equivalent)

\begin{tabular}{lccccc}
\hline & 1988 & 1996 & 2004 & 2012 & 2020 \\
$\begin{array}{l}\text { Gross Emissions from } \\
\text { Energy and Solid Waste }\end{array}$ & 314.7 & 299.0 & 286.9 & 275.6 & 272.5 \\
$\begin{array}{l}\text { Net Carbon Uptake from } \\
\text { Growing Forest Biomass }\end{array}$ & 25.6 & 26.8 & 28.2 & 29.8 & 31.4 \\
Net Emissions & 289.1 & 272.2 & 258.7 & 245.8 & 241.1 \\
\hline
\end{tabular}

In the next section, we maintain the energy demand assumptions built into the Conservation Case, and examine the impact of increasing the contribution of biomass to the fuel mix. 


\section{Moderate and Advanced Biomass Policy Scenarios to Reduce Greenhouse Gases}

In the last section we completed the first two scenarios, combining Current Biomass Policy with Reference Case and Conservation Case energy demand assumptions, respectively. In this section we develop two additional scenarios:

3) Moderate Biomass Policy Scenario

4) Advanced Biomass Policy Scenario

These scenarios incorporate the energy demand assumptions built into the Conservation Case, but rely on a successively greater contribution from biomass to displace fossil fuel combustion and otherwise mitigate the net emission of greenhouse gases. This section concludes with a comparison of summary results for all four scenarios.

Greenhouse gas emissions in the region can be significantly reduced by a variety of measures - end-use efficiency, advanced fossil technologies, fuel switching, and the use of renewables, each contributing a part of the overall reduction. Renewable energy production from the use of biomass is a readily available option to reduce net emissions of greenhouse gases by displacing fossil fuels with a resource that can remove as much carbon from the atmosphere as it releases.

The ability of biomass to reduce the accumulation of carbon dioxide in the atmosphere depends on the type and quantity of vegetation added to existing stocks or being protected from loss. In this section, we consider measures which add to existing stock, or which reduce the use of existing stocks. These approaches are all based on the same basic premise: addition to the planet's net carbon stores in vegetative matter will moderate global warming by keeping atmospheric carbon dioxide accumulations less than they would otherwise be.

Each of the options discussed here is capable of reducing net emissions but some are more efficient and less expensive than others. It is beyond the scope of this report to consider the costs of these options; rather this report identifies the amount of carbon emissions which can be reduced by pursuing each option.

The measures that are examined include:

- $\quad$ greater utilization of harvesting and processing losses;

- $\quad$ increased forest management;

- urban tree planting;

- $\quad$ short rotation crops; and

- increased efficiency biomass energy production. 


\subsection{Moderate Biomass Policy Scenario}

In this section our Moderate Biomass Policy Scenario is discussed: currently available technologies and biomass management practices are used to expand the use of biomass resources in the region, and to reduce overall net carbon releases. Existing and new wood-electric resources are used to meet about 3 percent of the total electric energy demand in the CONEG states by 2020 . This entails an increase of $2610 \mathrm{MW}$, the amount assumed by the D.O.E., from the current $806 \mathrm{MW}$ to $3416 \mathrm{MW}$ by 2020 . The three percent of total electric generation supplied by wood in this scenario is after the 28 percent of demand (including electricity) that we assumed would be met with electric energy conservation. Wood electric generation, together with other biomass electric generation - MSW and landfill methane recovery - gives a total biomass contribution of about 4.5 percent of electricity production by 2020 .

\section{Wood-Electric Power Supply}

Conventional wood-electric power supply facilities with characteristics typical of technologies in use today were assumed to enter service over the 1988-2020 period according to the schedule given in Table 6.1. The range of efficiencies that have been experienced and predicted for these facilities is about 14 to $20 \mathrm{MMBtu} / \mathrm{MWh}$. We assume here that the heat rate of the wood-electric facilities coming into service in the region will be $16.5 \mathrm{MMBtu} / \mathrm{MWh}$, and that the facilities will operate at an average capacity factor of 65 percent.

Table 6.1

Moderate Biomass Policy Wood-Electric Power Supply in the CONEG Region

(MW)

\begin{tabular}{lccccc}
\hline & 1988 & 1996 & 2004 & 2012 & 2020 \\
New England & 716 & 786 & 856 & 926 & 926 \\
Mid-Atlantic & 77 & 477 & 877 & 1277 & 1277 \\
New York & 13 & 413 & 813 & 1213 & 1213 \\
CONEG & 806 & 1676 & 2456 & 3416 & 3416 \\
\hline
\end{tabular}

To reach this objective over the next 30 years major policy changes must be made at both state and federal levels. In the Advanced Biomass Policy scenario that follows we expand our wood-electric targets to almost 6 percent of total electricity. Other more 
aggressive scenarios, such as no net increase or substantial decreases in greenhouse gas emissions from current levels, would require expansion of the initiatives proposed in this scenario, as well as other biomass measures more fully discussed in Section 7.

It should be remembered that a wide variety of renewable, efficient, and low or non- carbon based fuels in the electricity sector, as well as in other sectors, will likely be needed to realize the ambitious carbon reduction targets that may be required to help ensure climate stabilization. Thus, a biomass contribution of much more than 5 percent of electricity production may not be warranted.

\section{Increasing the Use of Residues}

Harvesting and milling residues provide a large potential source of fuel for energy production. There are four basic methods of logging, each of which is designed to accommodate a number of physical and economic factors peculiar to the logging site. These four methods result in different volumes of harvesting residue. Once the tree is felled, it can be:

- $\quad$ skidded to the roadside as a whole tree;

- $\quad$ delimbed and the top cut off, and the stem skidded to the roadside;

- delimbed, topped, and bucked into long logs which are skidded; or

- cut into shorter logs which are skidded.

The first option, whole tree skidding, brings out the most wood but can cause the most peripheral damage to the forest. The other methods produce higher harvesting residues but can cause less damage to the forest.

Whole tree chipping, a relatively new practice in the Northeast, produces the least harvesting residue. This method can be quite useful in the Northeast where trees are small and terrain moderate. Whole tree chipping results in very small amounts of harvesting I sidues; usually only the stump (about 5 percent) and smaller branches of the tree remain.

There are positive and negative environmental consequences from the removal of harvesting residues. If harvesting residues are routinely gathered and burned cleanly, residue use for energy production can be environmentally beneficial if it displaces the combustion of more polluting fossil fuels. On the other hand, fossil fuels that are relatively clean or can be combusted relatively cleanly, such as natural gas combined cycle, steam injected turbines and even some advanced coal technologies, might be preferable, but for the greenhouse gas problem. With climate stabilization goals, sustainably produced biomass - including the use of biomass residues - becomes an attractive option. 
Residue removal, however, does pose a danger of soil depletion. Although nutrient cycling is not well understood, there appears to be some danger from residue removal in short-rotation silviculture. Residues also play a number of environmentally bereficial roles as animal shelter and protection against erosion. Finally, residue removal is likely to exacerbate disturbances to the forest floor by damaging or killing other vegetation.

We have assumed modest increases in the use of forest harvest residues and milling residues relative to Current Biomass Policy, but we do not assume any increased use of dead wood which is needed within the forest ecology. The increased use of forest residues, we expect, will come as whole tree chipping operations spread across the Northeast. Referring to Tables 4.4 and 4.5 , we assume that harvesting losses are reduced from the 17.6 percent of total forest biomass in the Current Biomass Policy scenario to 9.7 percent in the Biomass Policy; thus, of the 38 percent of forest biomass that is not directly harvested, residue use increases from 42 percent to 68 percent.

Milling residues also provide a ready source of fuelwood for electricity production. Currently mill residues are used primarily for agricultural purposes and a variety of other uses. In the Current Biomass Policy scenario we assume the milling losses and uses of Table 4.5 , with about 35 percent lost at the mill and 48 percent of this used for fuelwood (12.2 percent of the original tree). In the Moderate Biomass Policy scenario, we have diverted most of the residues to fuelwood use. We have assumed that 80 percent of the residues created in the milling process would be used for fuelwood, or about 20 percent of the mass of the original tree. No increases in milling efficiency have been assumed.

\section{Increased Forest Management}

In the Moderate Biomass Policy Scenario we assume a 50 percent increase in the level of forest management, from about 100,000 acres per year to about 150,000 acres per year. Increased forest management would provide net yields about twice that currently occurring in the region. Thus, an additional 1.4 to 2.0 green tons/acre could be obtained. The limits to intensive forest management arise primarily from the high degree of small holdings in the region. If there is increased demand for fuelwood resources, then the incentive for better forest management would increase. At the same time it would require that acceptable environmental protection protocols be implemented.

\section{Results}

\section{Forest Biomass Stocks}

The increased use of biomass for wood-electric power supply is offset in part by the increased forest management and use of wood residues. in this scenario, there is a slight reduction in the growth of forest biomass stocks, relative to the Current Biomass 
Policy Scenario, with the Conservation Case energy assumptions. Nonetheless, as Table 6.2 shows, forest biomass stocks increase by about 7 percent by $? .020$ from current levels.

Table 6.2

Moderate Biomass Policy

- Conservation Case Energy .

Forest Biomass Stocks by CONEG Region

(Million green tons)

\begin{tabular}{lrrrrr}
\hline & 1988 & 1996 & 2004 & 2012 & 2020 \\
New York & 1,529 & 1,616 & 1,670 & 1,683 & 1,671 \\
Mid-Atlantic & 2,033 & 2,111 & 2,159 & 2,176 & 2,168 \\
New England & 2,732 & 2,731 & 2,754 & 2,800 & 2,869 \\
CONEG States & 6,295 & 6,458 & 6,584 & 6,660 & 6,708 \\
\hline
\end{tabular}

\section{Forest Biomass Yields}

Forest biomass yields, shown in Table 6.3, exhibit a pattern similar to stocks.

Table 6.3

Moderate Biomass Policy

- Conservation Case Energy -

Forest Biomass Yields by CONEG Region

(million green tons)

\begin{tabular}{lrrrrr}
\hline & 1988 & 1996 & 2004 & 2012 & 2020 \\
New York & 37.1 & 39.2 & 40.6 & 41.1 & 41.2 \\
Mid-Atlantic & 45.3 & 47.5 & 49.3 & 50.7 & 51.6 \\
New England & 63.9 & 66.0 & 68.5 & 71.7 & 74.3 \\
CONEG States & 146.3 & 152.6 & 158.4 & 163.5 & 167.0 \\
\hline
\end{tabular}




\section{Carbon Balances}

Table 6.4 summarizes net carbon uptake from growth less decay in the region's forests. Net carbon uptake in the Moderate Biomass Policy Scenario is higher than in the Current Biomass Policy Scenario case, despite the lower stocks and yields. This occurs as a result of the increased use of biomass residues and hence lower carbon releases from forest biomass decay. (These emissions show up elsewhere in the combustion of biomass for end-use energy and wood-electric power plants.) 
Table 6.4

Moderate Biomass Policy

- Conservatisn Case Energy -

Forest Carbon Uptake in the CONEG Region

(million tons)

GROSS CARBON UPTAKE

FROM FOREST GROWTH

$\begin{array}{lrrrrr} & 1988 & 1936 & 2004 & 2012 & 2020 \\ \text { New York } & 8.9 & 9.4 & 9.8 & 10.0 & 10.0 \\ \text { Mid Atlantic } & 11.1 & 11.7 & 12.2 & 12.6 & 12.8 \\ \text { New England } & 14.9 & 15.5 & 16.2 & 17.0 & 17.7 \\ & & & & & \\ \text { CON States } & 34.9 & 36.6 & 38.2 & 39.6\end{array}$

GROSS CARBON RELEASES

FROM FOREST BIOMASS DECAY

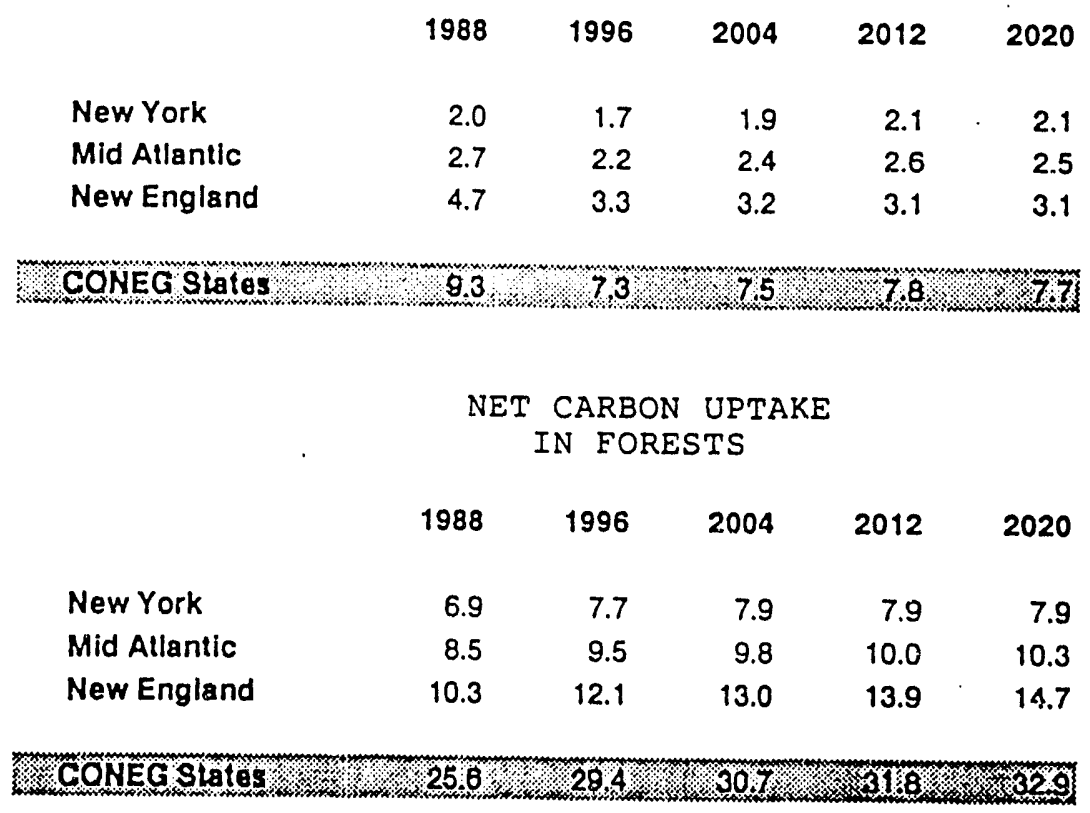


The maxituum carbon reduction benefit of wood-electric power supply will derive from displacement of coal generation, either existing or new. Table 6.5 summarizes the wood-electric power supply additions for the region in the Current Biomass Policy and the Moderate Biomass Policy scenarios. The $2600 \mathrm{MW}$ of wood-electric power supply added to the CONEG system in the Moderate Biomass Policy scenario contributes an additional $1740 \mathrm{MW}$ above the Current Biomass Policy scenario:

Table 6.5

Moderate Biomass Policy Capacity Additions (MW)

\begin{tabular}{|c|c|c|c|c|c|}
\hline \multicolumn{2}{|c|}{1988} & 1996 & 2004 & 2012 & 2020 \\
\hline Current Biomass Policy & 0 & 289 & 581 & 870 & 870 \\
\hline Moderate Biomass Policy & $\underline{0}$ & $\underline{870}$ & $\underline{1740}$ & $\underline{2610}$ & $\underline{2610}$ \\
\hline Net Change & 0 & 581 & 1159 & 1740 & 1740 \\
\hline \multicolumn{6}{|c|}{$\begin{array}{l}\text { Net Additional Carbon Emissions Wood-Electric Displacing Coal' } \\
\text { (Million Tons Carbon) }\end{array}$} \\
\hline & 0 & 0.7 & 1.3 & 1.9 & 1.9 \\
\hline
\end{tabular}

Overall carbon balances for the Moderate Biomass Policy Scenario are given in Table 6.6, below. While the use of forest and milling residues and forest management has been increased, this has been insufficient to offset the increased wood combustion. Thus, the net benefit from displacing coal in this scenario is negligible. To capture the full potential benefit, forest management would have to be increased well beyond the 50 percent assumed here.

\footnotetext{
${ }^{36} \mathrm{We}$ assume that the wood-electric power plant has a heat rate of $16.5 \mathrm{MMBtu} / \mathrm{MWh}$ a capacity factor of 65 percent, and emissions of $220 \mathrm{lb} / \mathrm{MMBtu}$. The coal plant is assumed to have a heat rate of 10.0 $\mathrm{MMBtu} / \mathrm{MWh}$ and the same emissions factor.
} 
Table 6.6

Carbon Balances for the CONEG Region

- Moderate Biomass Policy -

Conservation Case

(Million Tons Carbon Equivalent)

\begin{tabular}{lrccrr}
\hline Gross Emissions from & 1988 & 1996 & 2004 & 2012 & 2020 \\
Energy and Solid Waste & 314.7 & 299.7 & 288.2 & 277.5 & 274.4 \\
$\begin{array}{l}\text { Net Carbon Uptake from } \\
\text { Growing Forest Biomass }\end{array}$ & 25.6 & 29.4 & 30.7 & 31.8 & 32.9 \\
Net Emissions & 289.1 & 270.3 & 257.5 & 245.7 & 241.5 \\
\hline
\end{tabular}

\subsection{Advanced Biomass Policy Scenario}

This scenario incorporates additional initiatives, based upon emerging wood electric technology and biomass production techniques, and urban afforestation to reduce energy demand and to sequester carbon from the atmosphere.

\section{BIG STIG}

The burning of biomass can be utilized directly to provide heat, or indirectly to generate electricity. This section considers improvements in existing electric generation technology as a method to increase utilization of renewable biomass resources.

Currently under development in the U.S. (at General Electric Company in Schenectady N.Y.) and Europe is the BIG/STIG, a steam injected gas turbine, based on advanced jet engine technology, which is powered by biomass. A BIG/STIG is a biomass integrated gasifier/steam injected gas turbine which is an adaptation of the traditional steam injected gas turbine. The advantage of this configuration is that it can be adapted to small scale operations (e.g., 10 to 50MW) and thus reduces biomass transportation costs.

The STIG technology is in widespread use in the aerospace and power preduction industries. The biomass gasifier is an untested commercial scale technology but early tests indicate promise for this technology. The BIG/STIG is highly efficient, with efficiencies above 40 percent, and according to Larson cost-competitive with coal and other technologies (Larson, 1990). Other work, from Larson and R. Williams of 
Princeton, contains similar conclusions. The assumptions for the STIG technology are contained in Table 6.7 below.

A BIG/STIG project is currently under consideration in Bennington, Vermont. A $50 \mathrm{MW}$ facility is designed for the site with an annual wood consumption of 100,000 cords. The U.S.F.S. estimates that sufficient wood can be procured from the local region without reducing forest inventory or yields. The National Audubon Society (Cook, 1991) is promoting this project as a model of the use of biomass for energy production. Their support is contingent on comprehensive forest management plans, incorporation of forest management costs into energy costs, and regulation of forest practices by the State Fish and Wildlife Department.

$\begin{array}{lc}\text { Additional MW for CONEG region (MW) } & 2,600 \\ \text { Hours/YR } & 8760 \\ \text { Capacity Factor (CF) } & 75.00 \% \\ \text { Heat Rate (HR) } & 10.5 \\ \text { Total TBTU Needed (MW * Hours* CF * HR) } & 179 \\ \text { Heat Content of Wood (BTU/Dry Ton) } & 13.8 \\ \text { Wood Required Million Dry Tons } & \\ \bullet \quad M i l l i o n \\ \bullet\end{array}$

Since BIG STIGs, an emerging power plant technology, are projected to have a heat rate of about 9.5 to $10.5 \mathrm{MMBtu} / \mathrm{MWh}$ while conventional wood-electric plants currently in use around the U.S. have heat rates ranging from about 14 to 20 MMBtu/MWh, the use of conventional plants to provide the same electricity and fossil fuel displacement would require at least 40 percent more biomass fuel and land than BIG STIGS. Tnus, at a 75 percent capacity factor $1000 \mathrm{MW}$ of BIG STIG power facilities would require 62 to 69 trillion Btu (about 4.5 to 5 million dry tons or 8.4 to 9.3 
millicn green tons), while the same generation from conventional wood-electric facilities would require about 92 to 131 trillion Btu (about 6.7 to 9.5 million dry tons or 12.4 to 17.7 green tons).

In this case we assume that, in addition to the $2600 \mathrm{MW}$ of new conventional wood-electric power plants installed by 2010 , an additional 2600 MW of BIG STIGs is installed between 2000 and 2020. Total Wood-electric power supply in the region for this case is given in Table 6.8.

Table 6.8

Advanced Biomass Policy Wood-Electric Power Supply in the CONEG Region

(MW)

\begin{tabular}{lccccc}
\hline & 1988 & 1996 & 2004 & 2012 & 2020 \\
New England & 716 & 786 & 914 & 1389 & 1794 \\
Mid-Atlantic & 77 & 477 & 935 & 1740 & 2145 \\
New York & 13 & 413 & 871 & 1676 & 2081 \\
CONEG States & 806 & 1676 & 2720 & 4805 & 6020 \\
\hline
\end{tabular}

\section{Short Rotation Woody Crops}

Short rotation woody crops (SRWC) are energy crops that represent a promising feedstock for liquid fuels. Their high yields can provide a basis for conserving scarce productive land resources while providing economies of scale in biomass fuel costs. SRWC production is a plantation technique designed to promote high biomass yields at very young stand ages. According to Ranney at Oak Ridge National Laboratory, the most cost-effective combination of cultural management techniques for obtaining high yields includes the use of improved clonal material, intensive site preparatic 7 and weed control, some fertilizing, harvest cycles of five to eight years, and other factors (Strauss, no date).

Presently, there are no commercial SRWC installations in the CONEG states. Although the concept is well beyond the R\&D stage it remains in the pre-commercialization stage. Expected yields in the near term are on the order of 10 dry tons/acre/year (EPRI, 1990; Wright et al, 1989), or 20 green tons/acre/year. Currently, sample plots have yielded $5-8$ dry tons/acre/year. This varies depending on soil quality, water availability, etc. The species most commonly used is a hybrid poplar, and willow has also been used in some areas. Silver maple may also be considered in the Northeast. 
It is unlikely that many of the plantations for SRWC would be in the New England. In order to produce a competitive fuel, the focus has been to plant on retired or purchased agricultural land which is in short supply in New England. This is apparently not the case for New York and New Jersey, which have a fair amount of abandoned agricultural land that if left would revert to natural forest.

From a $\mathrm{CO}_{2}$ perspective, it may be unwise to clear an old-growth forest to plant SRWC. A recent (Harmon et al, 1990) paper in Science examines the $\mathrm{CO}_{2}$ accounts of old growth clearing to plant fast-growing trees. The results indicate that conversion of old-growth forests to young fast-growing forests will not decrease atmospheric $\mathrm{CO}_{2}$. This should not be surprising as old-growth forests have a lot of carbon tied up in the organic matter and humus.

This study has proposed plantations of SRWC to supply about 2 percent of the region's electric energy consumption, using the BIG STIG power plant technology. SRWC would be used in conjunction with increased usage of harvesting and milling residues. Using a yield assumption of 20 tons (freshweight) per acre per year, we estimate that a little over one million acres must be planted in the CONEG states to supply the necessary biomass.

The difference in land requirements for $1000 \mathrm{MW}$ of BIG STIG and conventional wood power plants (at 75 percent capacity factor), using either additional managed forest or SRWC resources is given in Table 6.9, below.

Table 6.9

Additional Land Required for Wood-Electric Power

(Thousand Acres)

\begin{tabular}{lrc} 
& $\begin{array}{r}\text { Managed Forest } \\
\text { (additional 2 tons/acre) }\end{array}$ & $\begin{array}{c}\text { SRWC Land Required } \\
\text { (20 tons/acre) }\end{array}$ \\
$\begin{array}{l}\text { Conventional } \\
\text { (@ 16.5 MMBtu/MWh) }\end{array}$ & 7,276 & 727 \\
BIG STIG & & 463 \\
(@ $10.5 \mathrm{MMBtu} / \mathrm{MWh})$ & 4,630 & 4 \\
\hline
\end{tabular}

Our Advanced Biomass Policy case assumes that 2600 MW of BIG STIG capacity would $b$. installed by 2020 , in addition to about $3400 \mathrm{MW}$ of conventional wood-electric power supply assumed in our Current Biomass Policy case, a rather large increase from the current $806 \mathrm{MW}$. For simplicity, the $2600 \mathrm{MW}$ of BIG STIG power plants in the 
CONEG region were assumed to be equally divided among the three regions - New England, Mid-Atlantic, and New York - and were assumed to phase in between 2000 and 2020 to provide time for the SRWC to be established.

Assuming an average size of $50 \mathrm{MW}$ this would entail the siting of 52 facilities throughout the region, preferably near the sources of fuelwood. The opportunities for establishing SRWC may be best in those areas of states where productive land is available, e.g., abandoned farmland in New York or Pennsylvania. To the degree that residues are relied upon for the wood fuel, proximity to forests and existing forest industry activities may be desirable. In any case, care would be required to ensure that local ecological constraints and conditions are satisfied; the dialogue between the Vermont DPU and environmental organizations to establish acceptable protocols for a proposed facility at Bennington provides a good starting point.

\section{Urban Trees}

Urban trees can reduce gross $\mathrm{CO}_{2}$ emissions in a number of ways. Two broad mechanisms can be classified; biological sequestering of $\mathrm{CO}_{2}$ and alteration of the urban microclimatic regime. Sequestering of $\mathrm{CO}_{2}$ during the growth phase of a tree can reduce the $\mathrm{CO}_{2}$ in the atmosphere by transferring carbon to the organic content of the tree. During the growth phase, the organic matter acts as a growing reservoir of carbon, displacing the atmospheric reservoir of $\mathrm{CO}_{2}$.

The second broad mechanism indirectly reduces $\mathrm{CO}_{2}$ emissions by lowering the demand for energy, typically supplied by fossil fuel burning power facilities. Urban trees significantly alter the energy balance of the urban environment by changing the radiative absorption properties of urban surfaces (shading), evapotranspiring water, and redirecting airflow. By reducing heat load, enhancing the atmospheric boundary layer, and lowering ambient air temperatures, reductions in energy demand associated with space cooling and heating in residential and commercial structures can be achieved. The impacts of these mechanisms are described in more detail below.

The first step in analyzing the potential $\mathrm{CO}_{2}$ offsets from potential urban forest growth is consideration of potential energy savings that can be expected from the addition of shade trees around residential and commercial structures. Estimated cooling load reductions are 11 to 43 percent and 4 to 28 percent for residential and commercial structures, respectively (Akbari et al, 1989). Estimated heating load reductions are 6 to 15 percent for average urban residential structures (Akbari, 1991).

We made estimates of residential urban shade tree planting potential for the CONEG states, based on the American Forestry Association survey of street trees in U.S. urban areas, which concluded that 11 percent of available planting spaces were available in New England and 44 percent in the Mid-Atlantic (including New York). This percentage was used as the fraction of households which would benefit from shade 
tree planting in our scenario, using the midpoint of the estimated range of savings noted above.

Table 6.10 gives an estimate of the total annual savings from reduced residential cooling and space heating needs due to the planting of urban shade trees for the New England, Mid-Atlantic, and New York regions. The potential in New England is lower than the other CONEG regions owing to the limited space availability. Approximately 14.7 million trees would be planted in this scenario.

Table 6.10

Potential Residential Energy Savings

From Urban Tree Planting

\begin{tabular}{|c|c|c|c|}
\hline & Savings (TBtu) & Resid Demand (TBtu) & $\%$ Savings \\
\hline New England & 3.81 & 565 & 0.67 \\
\hline Mid-Atlantic & 39.60 & 1320 & 3.00 \\
\hline New York & 22.90 & 718 & 3.19 \\
\hline \multicolumn{4}{|l|}{ CONEG } \\
\hline Natural Gas & 28.50 & 1160 & 2.45 \\
\hline Electricity & 11.00 & 603 & 1.83 \\
\hline Oil & 26.90 & $\underline{835}$ & $\underline{3.22}$ \\
\hline Total CONEG & 66.40 & 2600 & 2.55 \\
\hline
\end{tabular}

The reduction in $\mathrm{CO}_{2}$ emissions due to the displacement of existing fuel use resulting from the planting of urban shade trees has been quantified using the LEAP model. A base case was constructed for the Northeast assuming existing growth rates in energy demand and assumptions concerning future electricity supply options. This was constructed with a 32 year time horizon bringing the model result: to the year 2020 . As a perturbation to this base case, an urban tree scenario was constructed. In this scenario, $1 / 2$ of the 14.7 million shade trees were in place and fully effective by the year 2004 and the remainder reached full effectiveness by the year 2020 . The direct sequestering of $\mathrm{CO}_{2}$ was calculated based on the assumption that each tree can sequester, on average, $13 \mathrm{lbs}$ of carbon per year (Akbari et al, 1989).

In addition to shade trees planted to offset energy use on private plots, programs aimed at tree planting in open areas, parks and other public spaces have been included in the urban tree planting scenario. This scenario assumes a 22 percent increase per year 
over present planting rates which in Northeast urban areas is approximately 0.28 million trees planted per year (extrapolated from the national average). This planting rate was shared out within the Northeast by population with the additional reduction $(1 / 5$ of the national average) in New England due to reduced planting potential. This scenario would fulfill the Northeast's share of the America the Beautiful initiative giving rise to approximately 110 million new tree plantings by the year 2010 . The carbon reduction due to these additional trees is presented in Table 6.11. The energy savings associated with the alteration of the urban climate due to these 110 million additional trees has not been calculated.

Table 6.11

Total Annual Carbon Reduction Savings From Urban Tree Planting In the CONEG Region (Tons Carbon Equivalent)

\begin{tabular}{|lc|c|c|c|c|}
\hline & 1988 & 1996 & 2004 & 2012 & 2020 \\
\hline $\begin{array}{l}\text { New England Region: } \\
\text { energy displacement }\end{array}$ & 0 & 0 & 0.04 & 0.08 & 0.12 \\
direct sequester-shade trees & 0 & 0 & 0 & 0 & 0 \\
direct sequester-other urban trees & 0 & 0 & 0 & 0.04 & 0.23 \\
Mid-Atlantic Regions: & & & & & \\
energy displacement & 0 & 0.21 & 0.32 & 0.55 & 1.15 \\
direct sequester & 0 & 0.01 & 0.02 & 0.03 & 0.04 \\
direct sequester-other urban trees & 0 & 0.01 & 0.08 & 0.43 & 2.16 \\
New York State: & & & & & \\
energy displacement & 0 & 0.01 & 0.20 & 0.35 & 0.70 \\
direct sequester & 0 & 0 & 0.02 & 0.03 & 0.04 \\
direct sequester-other urban trees & 0 & 0 & 0.05 & 0.30 & 1.51 \\
Total & & & & & \\
energy displacement & 0 & 0.37 & 0.57 & 0.97 & 1.97 \\
direct sequester & 0 & 0.02 & 0.04 & 0.07 & 0.09 \\
direct sequester-other urban trees & 0 & 0.17 & 0.34 & 0.51 & 0.68 \\
TOTAL & 0 & 0.56 & 0.95 & 1.55 & 2.74 \\
\hline
\end{tabular}

The results in Table 6.11 indicate that carbon reduction from the displacement of energy far exceeds the carbon reduction from direct sequestering of $\mathrm{CO}_{2}$ by the urban shade trees. 


\subsection{Solid Waste Policy Results}

The current state policies for increased recycling, composting and source reduction - represented in our solid waste system analysis in Section 3 - entail rather strong shifts towards these new modes of solid waste management. Thus, despite a more than 40 percent increase in waste generation over the study period, net carbon equivaient emissions are expected to decrease by about 30 percent. Nonetheless, we have assumed that even greater levels of these options could be targeted as part of a greenhouse gas mitigation strategy for the region. In the Advanced Biomass Policy Scenario, we assume that each of the CONEG states would achieve 20 percent source reduction, 40 percent recycling, and 20 percent composting by the year 2020. Table 6.12 summarizes the results of these policy assumptions for the region. With these targets the carbon equivalent emissions from the solid waste systems would be reduced by about 65 percent from 1988 levels. A total of 6.8 million tons carbon equivalent reduction wculd be achieved by 2020 , relative to Reference Case projected emissions. About 78 percent of this, or 5.3 million tons carbon equivalent reduction, would come from landfill and composting, and 1.5 million from reduced MSW as some facilities retire or scale back operations with the reduced waste stream. 
Table 6.12

CONEG Region Solid Waste System

Greenhouse Gas Emissions

(million tons)

New England

$\begin{array}{lllll}1988 & 1996 \quad 2004 & 2012 \quad 2020\end{array}$

Landill Emissions

\begin{tabular}{llllll}
$\mathrm{CO} 2$ & 1.83 & 1.01 & 2.12 & 2.34 & 2.54 \\
$\mathrm{CH} 4$ & 0.75 & 0.20 & 0.00 & 0.00 & 0.00 \\
\cline { 2 - 6 } C Equin & 2.14 & 0.78 & 0.56 & 0.62 & 0.67
\end{tabular}

Res Recovery Emissions

$\mathrm{CO} 2$

$C$ Enuiv

Total C Equivalent

Mid Atlantic

Landfill Emissions

$\mathrm{CO} 2$
$\mathrm{CH} 4$
$\mathrm{C}$ Equin

Res Recovery Emissions

$\mathrm{CO} 2$

C Equin

Total C Equivalent

\begin{tabular}{lllll}
6.53 & 3.37 & 4.21 & 4.47 & 4.77 \\
2.20 & 0.50 & 0.10 & 0.05 & 0.03 \\
\hline 7.79 & 3.39 & 1.42 & 1.36 & 1.37
\end{tabular}

\begin{tabular}{lllll}
3.44 & 6.80 & 6.59 & 5.26 & 4.63 \\
\hline 0.94 & 1.85 & 1.80 & 1.43 & 1.26
\end{tabular}

8.73

5.24

3.22

2.80

2.63

New York

Landfill Emissions

$\mathrm{CO} 2$
$\mathrm{CH} 4$
$\mathrm{C}$ Equin

Res Recovery Emissions

$\mathrm{CO} 2$

C Equin

Total C Equivalem

\begin{tabular}{lllll}
4.71 & 3.98 & 4.48 & 4.14 & 4.14 \\
1.71 & 1.18 & 0.46 & 0.23 & 0.12 \\
\hline 5.50 & 4.29 & 2.46 & 1.75 & 1.46
\end{tabular}

\begin{tabular}{lllll}
2.95 & 3.40 & 3.40 & 3.40 & 3.40 \\
\hline 0.80 & 0.93 & 0.93 & 0.93 & 0.93
\end{tabular}

6.75

5.22

3.39

2.67

2.38

CONEG Region

Landfill Emissions

$\mathrm{CO}_{2}$
$\mathrm{CH}_{4}$
$\mathrm{C}$ Equin

Res Recovery Emissions

○2

$$
\text { . Equin }
$$

Total C Equivalent

\begin{tabular}{rrrrr}
13.07 & 8.36 & 10.81 & 10.96 & 11.45 \\
4.67 & 2.28 & 0.56 & 0.28 & 0.15 \\
\hline 15.89 & 8.46 & 4.45 & 3.73 & 3.50
\end{tabular}

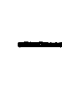

\begin{tabular}{rrrrr}
11.03 & 15.73 & 14.00 & 11.80 & 10.74 \\
\hline 3.01 & 4.29 & 3.82 & 3.22 & 2.93
\end{tabular}

18.90

12.75

8.27

6.95

6.43 


\section{Results}

The Advanced Biomass Policy case results in significant net uptake of greenhouse gases as well as the production of about 5 percent of the region's electricity from additional biomass sources.

\section{Forest Biomass Stocks}

As shown in Table 6.13 , total forest stocks increase by 10 percen: over the thirty year period, from 6296 million tons in 1988 to 6924 million tons by 2020. This brings stocks in the region to about 6 percent above that reached in the Current Biomass Policy Scenario, with the Reference Case Energy forecast, and about the same as with the Conservation Case forecast.

Table 6.13

Advanced Biomass Policy

- Conservation Case Energy -

Forest Biomass Stocks by CONEG Region

(million green tons)

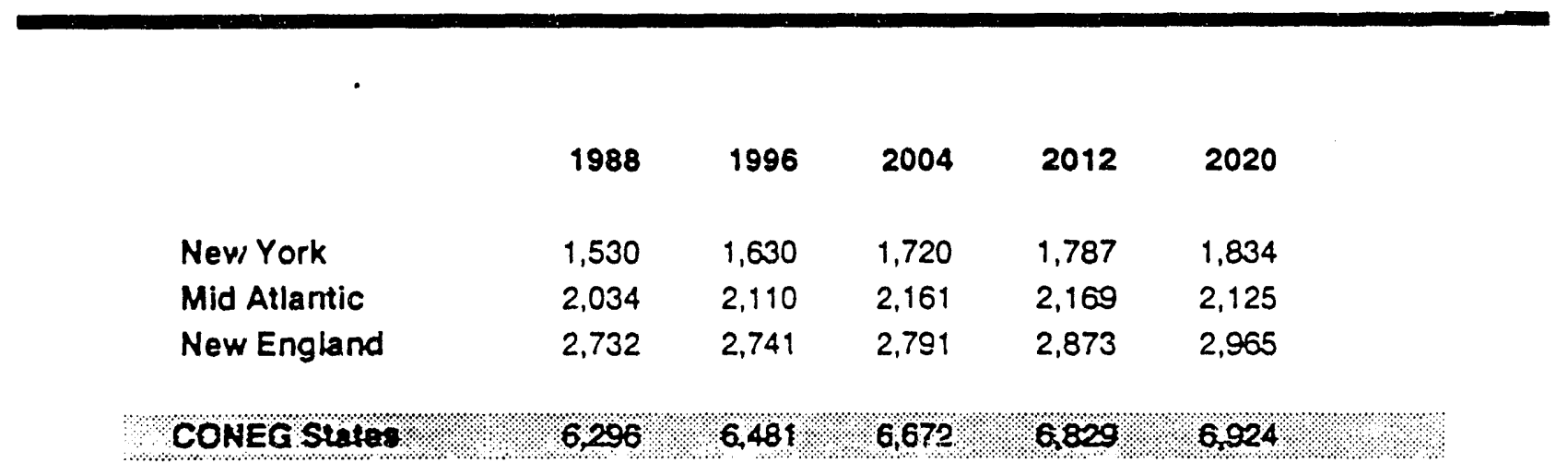

\section{Forest Biomass Yields}

Yields increase by 33 percent, from 146 million tons in 1988 to 194 million tons in this case, as shown in Table 6.14. By 2020, the annual yields are 20 percent above those of the Current Biomass Policy Scenario, with Reference Case energy forecast, and about 
13 percent above the yields in the Current Policy with the Conservation scenario energy forecast, despite the great increase in wood use for electric power supply. This occurs as a result of the various biomass production policies reflected in this case, including large scale planting of short rotation woody crops, urban tree planting, increased residue usage, and increased forest management.

Table 6.14

Advanced Biomass Policy

- Conservation Case Energy -

Forest Biomass Yields by CONEG Region

(million green tons)

\begin{tabular}{|c|c|c|c|c|c|}
\hline & 1988 & 1996 & 2004 & 2012 & 2020 \\
\hline New York & 37.18 & 41.43 & 47.48 & 51.55 & 55.88 \\
\hline Mid Atlantic & 45.31 & 417.68 & 51.01 & 52.75 & 54.39 \\
\hline New England & 63.94 & 67.77 & 73.79 & 78.98 & 83.85 \\
\hline CONEG Statos & 146.43 & 156.88 . & 172.28 & 18328 & $194: 12$ \\
\hline
\end{tabular}

\section{Carbon Balances}

Table 6.15 below provides gross releases and net uptake of carbon in the Advanced Biomass Policy Scenario. Net carbon uptake increases from about 28 million tons in 1988 to over 40 million tons in this scenario. Note that the carbon uptake in 2020 is more than 12 million tons above that in Current Biomass Policy, with Reference Case energy assumptions, thus offsetting increases in gross emissions from the increased wood-electric and BIG STIG power plant operation. 
Table 6.15

Advanced Biomass Policy

- Conservation Case Energy -

Forest Carbon Uptake and Fielease in the CONEG Region

\begin{tabular}{|c|c|c|c|c|c|}
\hline & \multicolumn{4}{|c|}{$\begin{array}{l}\text { GROSS CARBON UPTAKE } \\
\text { FROM FOREST GROWTH }\end{array}$} & \multirow[b]{2}{*}{2020} \\
\hline & 1988 & 1996 & 2004 & 2012 & \\
\hline New York & 8.9 & 10.1 & 11.7 & 12.8 & 14.0 \\
\hline Mid Atlantic & 11.1 & 11.8 & 12.7 & 13.2 & 13.6 \\
\hline New England & 14.9 & $i 6.0$ & 17.6 & 19.0 & 20.4 \\
\hline \multirow[t]{3}{*}{ CONEG States } & 34.9 & 37.8 & 42.0 & 45.0 & 48.0 \\
\hline & \multicolumn{5}{|c|}{ GROSS CARBON RELEASES } \\
\hline & 1988 & 1996 & 2004 & 2012 & 2020 \\
\hline New York & 2.0 & 1.7 & 1.9 & 2.1 & 2.1 \\
\hline Mid Atlantic & 2.7 & 2.2 & 2.3 & 2.5 & 2.5 \\
\hline New England & 4.7 & 3.3 & 3.2 & 3.1 & 3.1 \\
\hline \multirow[t]{3}{*}{ CONEG States } & 8.3 & 73 & 74 & 7.8 & 7.8 \\
\hline & \multicolumn{4}{|c|}{$\begin{array}{l}\text { NET CARBON UPTAKE } \\
\text { IN FORESTS }\end{array}$} & \\
\hline & 1988 & 1996 & 2004 & 2012 & 2020 \\
\hline New York & 6.9 & 8.3 & 9.8 & 10.7 & 11.9 \\
\hline Mid Allantic & 8.5 & 9.6 & 10.3 & 10 & 11.1 \\
\hline New England & 10.3 & 12.6 & 14.5 & 15.9 & 17.3 \\
\hline
\end{tabular}


The overall carbon balances for the Advanced Biomass Policy Scenario are given in Table 16, below.

Table 6.16

Carbon Balances for the CONEG Region

- Advanced Biomass Policy -

Conservation Case

(Million Tons Carbon Equivalent)

\begin{tabular}{lrrrrr}
\hline \multicolumn{1}{c}{1988} & $\mathbf{1 9 9 6}$ & $\mathbf{2 0 0 4}$ & $\mathbf{2 0 1 2}$ & $\mathbf{2 0 2 0}$ \\
$\begin{array}{l}\text { Gross Emissions from } \\
\text { Energy and Solid Waste }\end{array}$ & 314.7 & 297.3 & 283.3 & 272.0 & 267.1 \\
$\begin{array}{l}\text { Net Carbon Uptake from } \\
\text { Growing Forest Biomass }\end{array}$ & 25.6 & 30.5 & 34.6 & 37.2 & 40.2 \\
Net Emissions & $\mathbf{2 8 9 . 1}$ & $\mathbf{2 6 6 . 8}$ & $\mathbf{2 4 8 . 7}$ & $\mathbf{2 3 4 . 8}$ & $\mathbf{2 2 6 . 9}$ \\
\hline
\end{tabular}

\subsection{Comparison of Summary Results}

Tables 6.17 through 6.20 compare the summary forest stocks, summary forest yields, forest carbon uptake, and net carbon equivalent emissions results for the four scenarios:

1) Current Biomass Policy - Reference Case Energy

2) Current Biomass Policy - Conservation Case Energy

3) Moderate Biomass Policy - Conservation Case Energy

4) Advanced Biomass Policy - Conservation Case Energy

\section{Forest Impacts}

The impact of the greatly increased biomass cultivation on the economy and ecology of the Northeast can only be speculated about. The levels of land conversion proposed in the Advanced Biomass Policy scenario, although significant, are not likely to cause major environmental problems. The one million acres (estimated to be required 
for Short Rotation Woody Crops to supply electric power in this scenario) represent less than two percent of the CONEG region's land area of nearly 70 million acres. 
Table 6.17

Forest Stocks by CONEG Region for

Four Scenarios

(million green tons)

CURRENT BIOMASS POLICY.REFERENCE CASE ENERGY

$\begin{array}{lccccc} & 1988 & 1996 & 2004 & 2012 & 2020 \\ \text { New York } & 1,529 & 1,616 & 1,693 & 1,755 & 1,809 \\ \text { Mid Atlantic } & 2,033 & 2,100 & 2,149 & 2,177 & 2,182 \\ \text { New England } & 2,732 & 2,700 & 2,606 & 2,623 & 2,560 \\ & & & & & \\ \text { CONEG States } & 6,295 & 6,417 & 6,508 & 6,555 & 6,551\end{array}$

CURRENT BIOMASS POLICY CONSERVATION CASE ENERGY

\begin{tabular}{|c|c|c|c|c|c|}
\hline New York & 1,529 & 1,621 & 1,715 & 1,811 & 1,914 \\
\hline Mid Allantic & 2,033 & 2,108 & 2,185 & 2,267 & 2,351 \\
\hline New Engiand & 2,732 & 2,708 & 2,702 & 2,712 & 2,731 \\
\hline CONEG States & 6,295 & 6,437 & 6,602 & 6.789 & 6.996 \\
\hline
\end{tabular}

MODERATE BIOMASS POLICY CONSERVATION CASE ENERGY

\begin{tabular}{|c|c|c|c|c|c|}
\hline New York & 1,529 & 1,616 & 1,670 & 1,683 & 1,671 \\
\hline Mid Atlantic & 2,033 & 2,111 & 2,159 & 2.176 & 2,168 \\
\hline Hew England & 2,732 & 2,731 & 2,754 & 2,800 & 2,869 \\
\hline CONEG SLEIES & $6,295$. & 6.458 & 6,584 & 6,660 & 6,708 \\
\hline
\end{tabular}

ADVANCED BIOMASS POLICY CONSERVATION CASE ENERGY

\begin{tabular}{|c|c|c|c|c|c|}
\hline New York & 1,530 & 1,630 & 1,720 & 1,787 & 1,834 \\
\hline Mid Atlantic & 2,034 & 2,110 & 2,161 & 2,169 & 2,125 \\
\hline New England & 2,732 & 2,741 & 2,791 & 2,873 & 2,965 \\
\hline OANEG StateS & 6.296 & $648 \%$ & 6672 & 6.829 & 6.924 \\
\hline
\end{tabular}


Table 6.18

Forest Yields by CONEG Region for

Four Scenarios

(million green tons)

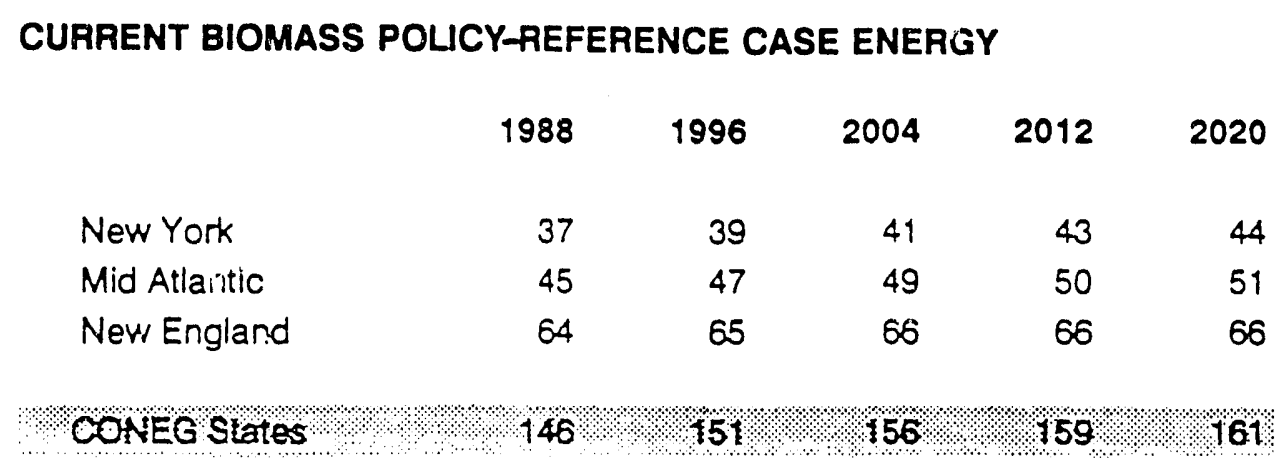

CURRENT BIOMASS POLICY-CONSERVATION CASE ENERGY

$\begin{array}{lllll}1988 & 1996 \quad 2004 & 2012 \quad 2020\end{array}$

$\begin{array}{llllll}\text { New York } & 37 & 39 & 42 & 44 & 46 \\ \text { Mid Atlantic } & 45 & 47 & 50 & 52 & 55 \\ \text { New England } & 64 & 65 & 67 & 68 & 70\end{array}$

GONEG States $/ 146 / 152$ ? $/ 158 \%$ ? $164 \%$ ? 17

MODERATE BIOMASS POLICY-CONSERVATION CASE ENERGY

$\begin{array}{lllll}1988 & 1996 & 2004 & 2012 & 2020\end{array}$

$\begin{array}{llllll}\text { New York } & 37 & 39 & 41 & 41 & 41 \\ \text { Mid Atlantic } & 45 & 47 & 49 & 51 & 52 \\ \text { New England } & 64 & 66 & 69 & 72 & 74\end{array}$

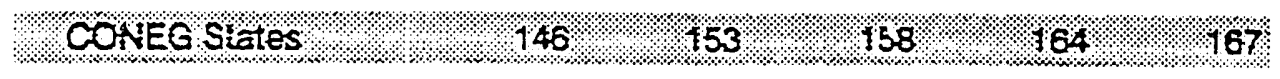

ADVANCED BIOMASS POLICY-CONSERVATION CASE ENERGY

$\begin{array}{lllll}1988 & 1996 \quad 2004 & 2012 \quad 2020\end{array}$

$\begin{array}{llllll}\text { New York } & 37 & 41 & 47 & 52 & 56\end{array}$

$\begin{array}{llllll}\text { Mid Atlantic } & 45 & 48 & 51 & 53 & 54\end{array}$

$\begin{array}{llllll}\text { New England } & 64 & 68 & 74 & 79 & 84\end{array}$

CoNEG States : : 
Table 6.19

Net Carbon Uptake by Region for

Four Scenarios

(million tons)

\begin{tabular}{|c|c|c|c|c|c|}
\hline \multicolumn{6}{|c|}{ CURRENT BIOMASS POLICK-REFERENCE CASE ENERGY } \\
\hline & 1988 & 1996 & 2004 & 2012 & 2020 \\
\hline New York & 6.9 & 7.4 & 7.6 & 7.8 & 8.1 \\
\hline Mid Atlantic & 8.5 & 3.7 & 8.9 & 9.0 & 9.0 \\
\hline New England & 10.3 & 10.5 & 10.7 & 10.7 & 10.4 \\
\hline CONEG Sutes & 25.6 & 26.5 & 27.1 & 27.5 & 27.5 \\
\hline
\end{tabular}

CURRENT BIOMASS POLICKCONSERVATION CASE ENERGY

$\begin{array}{lrrrrr} & 1988 & 1996 & 2004 & 2012 & 2020 \\ \text { New York } & 6.9 & 7.4 & 7.8 & 8.3 & 9.0 \\ \text { Mid Atlantic } & 8.5 & 8.9 & 9.3 & 9.9 & 10.5 \\ \text { New England } & 10.3 & 10.6 & 11.1 & 11.6 & 12.0 \\ \text { CoNEG States } & & & & & \\ \text { o. } & 25.6 & 28.8 & 28.2\end{array}$

MODERATE BIOMASS POLICY-CONSERVATION CASE ENERGY

\begin{tabular}{|c|c|c|c|c|c|}
\hline & 1988 & 1996 & 2004 & 2012 & 2020 \\
\hline New York & 6.9 & 7.7 & 7.9 & 7.9 & 7.9 \\
\hline Mid Atlantic & 8.5 & 9.5 & 9.8 & 10.0 & 10.3 \\
\hline New England & 10.3 & 12.1 & 13.0 & 13.9 & 14.7 \\
\hline CONEG SLETES & 25.6 & 29.4 & 30.7 & 318 & 32.9 \\
\hline
\end{tabular}

\section{ADVANCED BIOMASS POLICY-CONSERVATION CASE ENERGY}

\begin{tabular}{|c|c|c|c|c|c|}
\hline & 1988 & 1996 & 2004 & 2012 & 2020 \\
\hline New York & 6.9 & 8.3 & 9.8 & 10.7 & 11.9 \\
\hline Mid Atlantic & 8.5 & 9.6 & 10.3 & 10.6 & 11.1 \\
\hline New England & 10.3 & 12.6 & 14.5 & 15.9 & 17.3 \\
\hline CONEG States & 25.7 & 30.5 & 34.6 & 372 & 402 \\
\hline
\end{tabular}




\section{Net Carbon Equivalent Emissions}

Table 6.20 summarizes the combined emissions results for energy, solid waste, and forestry (including urban trees) from all four scenarios.

As noted earlier, annual carbon equivalent emissions decrease by about 17 percent from 1988 to the year 2020 in the Conservation Case (Current Biomass Policy). By the year 2020, this represents a 33 percent reduction in carbon equivalent emissions relative to the Reference Case ( 241.1 million tons versus 361.7 million tons).

Note that there is almost no net benefit from the wood-electric facilities which displace $1740 \mathrm{MW}$ of coal-fired power in the Moderate Biomass Policy Scenario. The addition of conventional wood-electric power supply, along with a 50 percent increase in forest management and increased use of forest and harvesting residues, has little effect on the region's overall carbon balances.

The higher heat rate relative to coal capacity results in net emissions of carbon when coal generation is displaced by conventional wood-electric generation. Improving the heat rate of wood-electric generation would close this gap.

At the same time, increasing forest management by 50 percent (50 thousand acres per year) brings an additional 3.4 million acres under management by 2020, with about 2 green tons per acre additional yield. This takes up about 1.7 million tons of carbon per year, about one-third of the carbon released from wood combustion. In addition, about 5.6 million green tons from increased use of forest and milling residues contributes to wood supply for the additional wood-electric facilities, and taking up about 1.4 million tons of carbon. Together, increased forest management and increased use of residues provide about two-thirds of the additional fuel needed for the wood-electric power plants, with the remaining 7.2 million green tons taken from current growth. Further increases in management, although difficult given the land ownership patterns, would be required to make up the difference.

Finally, with BIG STIGs displacing coal generation, supplied by about 1 million acres of short rotation woody crops, urban forestry, and ambitious solid waste management strategies for the states in the region, about 14.6 million tons additional carbon reduction is achieved in the Advanced Biomass Policy scenario. About 5.3 million tons come from solid waste strategy, about 2 million from urban shade trees, and about 7.3 million come from short rotation crops and related forest biomass impacts. ${ }^{37}$ This adds about 4 percent to the 33 percent achieved by energy conservation.

\footnotetext{
${ }^{37}$ Of the 7.3 million tons increase in net carbon uptake embodied in changing from the Moderate to the Advanced Biomass scenario, almost 1 million is from carbon uptake from urban trees.
} 
Table 6.20

\section{Carbon Balances for the CONEG Region \\ Four Scenarios \\ (Million Tons Carbon Equivalent)}

CURRENT BIOMASS POLICY-REFERENCE CASE ENERGY

$\begin{array}{lccccr}\begin{array}{l}\text { Gross Emissions } \\ \text { Froin Energy } \\ \text { and Solid Waste }\end{array} & 1988 & 1996 & 2004 & 2012 & 2020 \\ \begin{array}{l}\text { Net Carbon Uptake } \\ \begin{array}{l}\text { From Growing Forest } \\ \text { Biomass }\end{array}\end{array} & 314.7 & 324.2 & 341.4 & 361.2 & 389.2 \\ \text { Net Emissions } & 25.6 & 26.5 & 27.1 & 27.5 & 27.5 \\ & 289.1 & 297.7 & 314.3 & 333.7 & 361.7\end{array}$

CURRENT BIOMASS POLICY-CONS'ERVATION CASE ENERGY

\begin{tabular}{|c|c|c|c|c|c|}
\hline Gross Ernissions from & 1988 & 1996 & 2004 & 2012 & 2020 \\
\hline $\begin{array}{l}\text { Energy and Solid Waste } \\
\text { Net Carbon Uptake from }\end{array}$ & 314.7 & 299.0 & 286.9 & 275.6 & 272.5 \\
\hline Growing Forest Biomass & 25.6 & 26.8 & 28.2 & 29.8 & 31.4 \\
\hline Net Emissions & 289.1 & 272.2 & 258.7 & 245.8 & 241.1 \\
\hline \multicolumn{6}{|c|}{ MODERATE BIOMASS POLICY-CONSERVATION CASE ENERGY } \\
\hline Gross Emissions from & 1988 & 1996 & 2004 & 2012 & 2020 \\
\hline Energy and Solid Waste & 314.7 & 299.7 & 288.2 & 277.5 & 274.4 \\
\hline $\begin{array}{l}\text { Net Carbon Uptake from } \\
\text { Growing Forest Biomass }\end{array}$ & 25.6 & 29.4 & 30.7 & 31.8 & 32.9 \\
\hline Net Emissions & 289.1 & 270.3 & 257.5 & 245.7 & 241.5 \\
\hline \multicolumn{6}{|c|}{ ADVANCED BIOMASS POLICY-CONSERVATION CASE ENERGY } \\
\hline & 1988 & 1996 & 2004 & 2012 & 2020 \\
\hline \multicolumn{6}{|l|}{ Gross Emissions from } \\
\hline Energy and Solid Waste & 314.7 & 297.3 & 283.3 & 272.0 & 267.1 \\
\hline \multicolumn{6}{|l|}{ Net Carbon Uptake from } \\
\hline Growing Forest Biomass & 25.6 & 30.5 & 34.6 & 37.2 & 40.2 \\
\hline Net Emissions & 289.1 & 266.8 & 248.7 & 234.8 & 226.9 \\
\hline
\end{tabular}




\section{Policies for Reducing Greenhouse Gas Emissions}

\subsection{Introduction}

An effective strategy for reducing net emissions of greenhouse gases in the CONEG region should be designed around several key factors:

- Identification of the largest sources (and potential sinks) of greenhouse gases in the region;

- Identification of those sources (sinks) for which technically feasible means of reduction (augmentation) exist or are likely to be developed in the near term;

- Identification of those options with the most favorable benefit to cost ratios (i.e., cost per ton of carbon emissions reduced);

- Identification of sound institutional bases for promulgating and implementing the strategy and its various elements.

These factors are perhaps self-evident, but important to recall and use as an initial screening framework, as strategies and associated policies are considered. For example, the first is especially compelling in light of the very ambitious carbon emissions reduction goals - e.g., 25 percent by 2005 and 50 to 70 percent by 2020 - that rire arguably required for climate stabilization. Those options that rank high on all of these factors should draw special attention; an option that is technically feasible, but is very costly and has a very small impact would not be high on the strategy hierarchy. Nonetheless, some attention should also be paid to forward-looking elements of a strategy: e.g., an option that could effect large reductions, but needs research, development and demonstration before it can be effectively implemented. Also, small contributions can be important if a large number can be implemented with a single option or policy.

Additional considerations must also come into play in fashioning. a sound strategy and associated policies. These include other environmental goals, including the limitation of air pollutant emissions that can adversely affect human health and the environment at local and regional levels, and competing economic and ecological constraints and objectives. Opportunities for cross-cutting strategy elements and policies should be sought, in order to realize economies in technology and administrative costs. Finally, it should be emphasized that with ambitious greenhouse gas reduction goals it may be necessary to piece together many strands of a strategy, of elements ranging in both the size and cost of their impact. 
Based upon these considerations the energy sector, the solid waste sector, and forestry all have important roles to play in an integrated greenhouse gas mitigation strategy for the region. As noted in Sections 2 and 3:

- Carbon dioxide from energy use contributes almost 80 percent of U.S. and CONEG region greenhouse gas global warming potential, and other greenhouse gas emissions from energy use also contribute;

- Methane from both energy activities and landfill contributes between about 5 percent and 15 percent, depending upon the assumptions used in computing its carbon equivalent global warming potential; and

- $\quad$ Forest growth in the CONEG region provides an uptake of carbon that offsets about 8 percent of gross annual carbon emissions.

The focus here, then, is on these three sectors in general, and on the role of biomass in particular. In each, attention should be drawn to those options that have favorable characteristics within the framework given above, including aggressive implementation of no-cost or low-cost options, and implementation of options that have combined or synergistic benefits.

\section{Energy}

Energy use - fossil fuel use in particular - is the major source of greenhouse gas emissions in the U.S. and the CONEG region. In the energy sector, end-use efficiency (demand-side management, or DSM) is particularly attractive: it can reach all sub-sectors and fuels which together dominate greenhouse gas emissions accounts; it can cut deeply into overall greenhouse emissions at essentially no net cost of equivalent carbon reduction; more expensive DSM can be targeted at high carbon emissions sectors and fuels; and its implementation can create jobs and stimulate the economy. Other potentially attractive strategy options in the energy sector include:

- $\quad$ Cogeneration (using fuel for dual purposes);

- Fuel switching to less carbon-intensive fuels;

- $\quad$ Changing operating practices (e.g., shifting power plant dispatch to use less polluting plants);

- Improving the efficiency of energy supply technologies; and

- Shifting modes of transport, spatial organization, product and process mix, to effect lower energy use, and carbon releases, consistent with other economic and societal goals. 
Among the potential energy sector options are efficiency and fuel switching in space conditioning, and efficiency in equipment and lighting, in the buildings sector; efficiency (fuel economy), fuel switching, and mode shifting in the transportation sector; advanced fossil technologies (e.g., natural gas fuel cells) and fuel switching in the electric sector; and expanded use of renewable energy resources, including biomass resources, in a variety of sectors.

Increased energy efficiency can take us a long way toward reducing dependence on costly, depletable and polluting resources. The remaining fuel needs for international, national and local economies would remain substantial, however, and it remains desirable to meet those needs with renewable, relatively clean resources, at reasonable costs. While some advanced highly efficient fossil technologies might be a suitable part of an overall mix, greater use of renewable resources, including biomass with no net carbon emissions - can help meet climate stabilization goals and other societal goals. To achieve this, however, such goals - reduced dependence on depletable fuels, economical energy services, environmental sustainability, and climate stabilization - must be embodied in policy, programs, planning, regulation and pricing, at the federal, regional, and state levels.

Although only modest progress has been made on the federal level in the past 12 years (since the PURPA legislation of 1978), state regulatory commissions have been making significant strides in incorporating energy efficiency oujectives, increasing small (and renewable) power supplies and, more recently, incorporating environmental objectives into resource acquisition protocols, under the rubric of least-cost integrated planning or integrated resource planning (IRP). Among the environmental objectives that have been included in some these policy and regulatory initiatives is the reduction of greenhouse gas releases to the atmosphere from energy and other anthropogenic sources.

IRP processes and resource bidding systems rave been instituted in several of the CONEG states, as in other states around the U.S., to encourage investments in energy efficiency and independent power production (IPP). While the original intent of the PURPA legislation was to encourage small power producers to provide cogeneration and renewable sources of electric power, more recently the IPP resource acquisition processes have been expanded to include all sources of power supply in order to encourage competition and lower electricity costs. Nonetheless, renewable resources can still compete with more traditional and advanced fossil resources in such a process, especially if environmental goals are taken into account.

Several recent utility regulatory developments may contribute to increasing the likelihood that energy efficiency and renewables will compete with more conventional power supply options. These are: the decoupling of electric energy sales and rates to remove disincentives to utility DSM investments, the creation of various incentive systems for utility DSM investments, the inclusion of DSM along with powe: supply in resource bidding systems, more thoroughgoing IRF proceedings with regulatory scrutiny, and the inclusion of environmental externalities costs in IRP processes. In California, Nevada, Massachusetts, New Jersey, and New York, for example, IRP processes and bidding systems have been instituted, using environmental externalities costs in resource 
selection. For $\mathrm{CO}_{2}$ in particular, the value currently adopted in Massachusetts and Nevada is about $\$ 24$ per ton, which implies about 2.5 cents per $\mathrm{kWh}$ added cost for a new coal plant and about 1.0 cents per $\mathrm{kWh}$ for a new natural gas combined cycle plant. These are significant additions to the costs of electricity from those resources and will render both DSM and renewables more competitive since they have no $\mathrm{CO}_{2}$ emissions.

The regulatory and policy activity summarized above has focussed on the regulated energy industry, and electricity in particular. To be sure, about forty percent of current $\mathrm{CO}_{2}$ emissions in the U.S. occur in the electric utility sector, but fossil fuel combustion in the buildings, transportation and industrial sectors, methane releases from landfill and coal mines, and CFC releases, also contribute to greenhouse warming potential. Thus, policies directed at these other sectors and sources of greenhouse gas releases need to be developed.

For energy combustion itself, the reflection of environmental externalities costs in investment and consumption decisions across all sectors along the full fuel chain (from extraction to processing to final fuel consumption) would require a comprehensive and consistent framework, e.g. pollution taxes or, as with the new Clean Air Act Amendments for sulphur dioxide, system quantity limits and a trading system in marketable pollution permits. Ideally, such a system would be established at the national level, but states could take the initiative - and some already have - in lieu of, and as a stimulant to, federal action.

\section{Solid Waste}

Strategies affecting the solid waste sector should also be included in solutions to the greenhouse gas problem. Her too, biomass plays a role - indeed a central one as a large fraction of the solid waste stream is organic carbon from forests (paper and wood products) and agriculture that is released as carbon dioxide and methane. Given the high global warming potential carbon equivalency of methane (10 to 30 times $\mathrm{CO}_{2}$, or about 2.7 to 8 in terms of $C$ ), the fact that one half the carbon released in anaerobic decomposition of organic matter in landfill is emitted as methane, attention to the solid waste stream is important. Looking back at the framework and related considerations for the development of greenhouse gas strategies for the region, the conditions and opportunities in solid waste are compelling.

First, solid waste has important relationships with the other key sectors involved in greenhouse gas releases and mitigation strategy. In particular, owing in part to its biomass content, solid waste can provide an energy resource to displace fossil fuels, with no net carbon releases if the organic matter that enters the waste stream is matched by re-growth (as in agriculture). Second, a large part of the solid waste stream that derives from forest products, which are produced in industries that use biomass both as their basic material and as an energy resource. For example, the use of residues especially in cogeneration applications can be an attractive option, both for its energy efficiency and no-net-carbon potential. Moreover, as these industries utilize the forest biomass resources they can degrade or improve the carbon uptake and sequestration offset to gross releases, depending upon their practices. 
Finally, it should be noted that, particularly in densely populated urban areas scarce and expensive land and concern about local pollutant emissions have motivated other strategies and policies, specific to solid waste, that intersect with the greenhouse gas mitigation issue. These include recycling, source reduction, and composting, in addition to waste to energy conversion. These options can often offer multiple benefits at net savings or little net cost - land-use conservation, local pollution reduction, greenhouse gas mitigation, and local jobs and economic stimulation. Re-cycling, source reduction, and composting in the solid waste sector thus bear strong similarities to DSM and cogeneration in the energy sector.

\section{Forestry}

Forest practices can also have multiple benefits - affecting forest products, energy use, recreation, greenhouse gas releases and uptake, jobs and regional economic benefits - that can be combined in designing an acceptable greenhouse gas mitigation strategy. Among the options that may have these features are: use of forest biomass for energy (particularly with cogeneration) in the forest industries, expansion of wood-electric power supply, improvement and expansion of forest management, preservation and increase of forest land, planting of trees in under-utilized or otherwise favorable locations, and the stimulation of research, development and demonstration both regionally and nationally.

At the same time, attention should be given to those societal goals that may come into conflict with such biomass development options - for example, competing land uses and ecological sustainability. With sufficient care, these factors can be taken into account, thereby both limiting the potential and requiring additional actions and investments to realize the maximum desirable.

\section{Summary of Biomass Options for the Reduction of Greenhouse Gas Emissions}

Appropriate biomass strategies focussed on the greenhouse gas problem would consider a mix of the following elements:

- Elimination or reduction of deforestation;

- Afforestation;

- Improved forest management;

- Substitution of wood for fossil fuels;

- More efficient wood-to-energy conversion technologies;

- Elimination or reduction of landfill gas releases through source reduction, recycling, composting, and methane capture for energy;

- Substitution of new wood resources for other materials; and

- Development of high yield woody and herbaceous crops.

Other, related, efforts would be appropriate, such as:

- Development of ecologically sound biomass resource production procedures; 
- $\quad$ Protection of wildlife habitats;

- $\quad$ Abatement of other air pollutants from biomass energy use; and

- Development of more advaried wood combustion technologies.

Each of these would be supported by energy, land-use, forestry, solid waste, and environmental policies and programs at the federal, regional and state levels. To further promote the development of renewable energy, this report proposes several broad policy initiatives for the CONEG states.

\section{Costs of Carbon Reduction Options}

Below we compare some cost trade-offs for various options in the energy, solid waste and forestry sectors.

\section{Energy}

In Table 7.1 the costs of new integrated gasification combined cycle coal, new natural gas combined cycle, existing oil-fired generation, BIG STIG, and conventional wood-electric power plants are listed, using fuel prices from the U.S. D.O.E. and technology costs and characteristics from the D.O.E., EPRI and the recent study America's Energy Choices. Note that we have assumed that wood will cost as high as $\$ 3.00$ per MMBtu, which may be somewhat high compared to today's costs. This has been done to account for the potential additional costs of providing substantial amounts of woody biomass with sound environmental practices. 
Table 7.1

Biomass/Fossil Cost Trade-offs

Electric Sector

2000 In-Service Date (cents per kwh)

\begin{tabular}{|c|c|c|c|c|c|}
\hline & IGCC & NGCC & OIL & BIG/STIG & WOOD \\
\hline Heat Rate & 9.20 & 7.74 & 10.00 & 10.50 & 16.5 \\
\hline Cap Cost $(1990 \$ / \mathrm{kw})$ & 1738 & 568 & $\cdots$ & $1000-1600$ & 1500 \\
\hline Cap Cost $(1990 \mathrm{c} / \mathrm{kw})$ & 2.65 & 0.87 & $\cdots$ & $1.52-2.44$ & 2.29 \\
\hline Fuel Cost $(1990 \mathrm{c} / \mathrm{kwh})$ & 1.95 & 4.71 & 6.38 & $2.10-3.15$ & $3.30-4.95$ \\
\hline O\&M Cost (1990c/kwh) & 0.91 & 0.46 & 0.40 & $0.70-1.00$ & 0.50 \\
\hline Other Cost & 0.46 & 0.46 & $\cdots$ & 0.46 & 0.46 \\
\hline Total Cost & 5.97 & 6.50 & 6.78 & $4.78-7.05$ & $6.55-8.20$ \\
\hline $\begin{array}{l}\mathrm{CO}_{2} \text { Emissions } \\
\text { (Tons/MWh) }\end{array}$ & 0.91 & 0.46 & 0.85 & 0.00 & 0.00 \\
\hline
\end{tabular}

Notes: Al costs in levelized real 1990 dollars at the point of generation. Assume 75 percent capacity factor, 10 percent levelized real fixed charge factor. Fossil fuel costs from EIA 1990 Annual Energy Outlook. Capital costs from EPRI Technical Assessment Guide. "Other cost" is estimate for $\mathrm{NO}_{x}$ pollution controls (assuming wood plants have same control costs as coal).

Switching from coal to natural gas reduces carbon dioxide at about $\$ 12$ per ton, while new efficient natural gas can reduce carbon at essentially no cost when displacing oil. The cost of net $\mathrm{CO}_{2}$ reduction is free if $\mathrm{BIG} / \mathrm{STIG}$ replaces an Integrated Gasification Combined Cycle Coal (IGCC) plant and ranges between a negative cost and about $\$ 12$ per ton $\mathrm{CO}_{2}$ if it replaces $\mathrm{NGCC}$ (in 1990 dollars). For wood steam plants displacing coal would reduce carbon dioxide at about $\$ 6.40$ to $\$ 25$ per ton. Moreover, owing to the heat rate difference, the STIG would require about one-third less wood resources (and hence less land), assuming the same sources of wood. The cost of reducing carbon dioxide by displacing gas with wood is higher, as gas has less carbon dioxide emissions: for BIG STIGs it ranges from a negative cost to $\$ 12$ per ton, and for wood steam plants it is between zero and about $\$ 37$ per ton. Finally, the cost of displacing existing oil steam plants with wood-electric are up to about $\$ 2$ and $\$ 17$ per ton for BIG STIG and conventional wood-steam respectively.

A comparison of power supply carbon reduction options is given below: 

Replace New Gas With BIG STIG
Replace New Coal With BIG STIG
Replace New Coal Gas
Replace New Coal With WOOD
Replace Existing Coal With Big STIG
Replace New Gas With WOOD
Replace Existing Coal With New NGCC
Replace Existing Coal With
Existing Gas ${ }^{38}$

- $\$ 37.4 /$ Ton to $+\$ 12.0 /$ Ton

- $\$ 13.1 /$ Ton to $+\$ 11.9 /$ Ton

$+\$ 11.8 /$ Ton

$+\$ 6.4 /$ Ton to $+\$ 24.0 /$ Ton

$+\$ 16.0 /$ Ton to $+\$ 41.2 /$ Ton

$0.0 /$ Ton to $+\$ 37.0 /$ Ton

$+\$ 65.0 /$ Ton

$+\$ 80.0 /$ Ton

The Oregon Department of Energy has made estimates of the net carbon dioxide reduction costs of various options. Using somewhat different assumptions (e.g., a 6.5 percent fixed charge rate, lower gas costs) they find:

\author{
Replace Existing Coal With Geothermal \\ Replace Existing Coal With NGCC \\ Replace Elec With Gas Water Htr \\ Other End-Use Fuel Switch \\ New Solar Photovoltaic \\ Replace Existing Coal With Conservation \\ at $<3 c / k W h$ )
}

\author{
$\$ 13 /$ Ton \\ $\$ 25 /$ Ton to $\$ 40 /$ Ton \\ $\$ 0 /$ Ton to $\$ 18 /$ Ton \\ $<\$ 40 /$ Ton \\ $\$ 34 /$ Ton \\ $<\$ 3 /$ Ton
}

The national energy strategy study America's Energy Choices, found that residential energy conservation up to 20 to 25 percent of consumption by 2010 , and 30 to 40 percent by 2030 , could be achieved at less than the avoided fuel costs, and thus at no cost per ton of carbon dioxide reduction. Similar reductions at less than avoided cost were found for the industrial and transport sectors. In the most ambitious scenario - Climate Stabilization - overall energy requirements were halved by conservation by 2030 , while renewable energy was increased more than four-fold. The highest cost options used to achieve that target were about $\$ 25 /$ Ton.

\footnotetext{
${ }^{38}$ Assume both have beat rates of $10 \mathrm{MMBtu} / \mathrm{MWh}$, and the displacement takes place in 2000 through a shift in dispatch.
} 


\section{Forestry}

Creating additional forest biomass can be used to provide feedstocks for wood energy facilities, or simply to remove carbon dioxide fium the atmosphere and sequester it for many years.

The World Resources Institute's (WRI) Minding the Carbon Store: Weighing U.S. Forestry Strategies to Slow Global Warming (Trexler, 1991), summarizes its recommended carbon storage options. The cost of these recommendations range from $\$ 8$ to $\$ 20 /$ Ton for urban tree planting, to $\$ 54$ to $\$ 272 /$ Ton for implementing a forestry reserve program. The table below adapts Table 3. of the WRI study, by expressing the costs in $\$ /$ Ton of $\mathrm{CO}_{2}$ rather than $\$ /$ Ton $\mathrm{C}$.

Table 7.3

Costs of Forestry Options for Carbon Storage/Reduction

(Costs Per Ton of Carbon Dioxide)

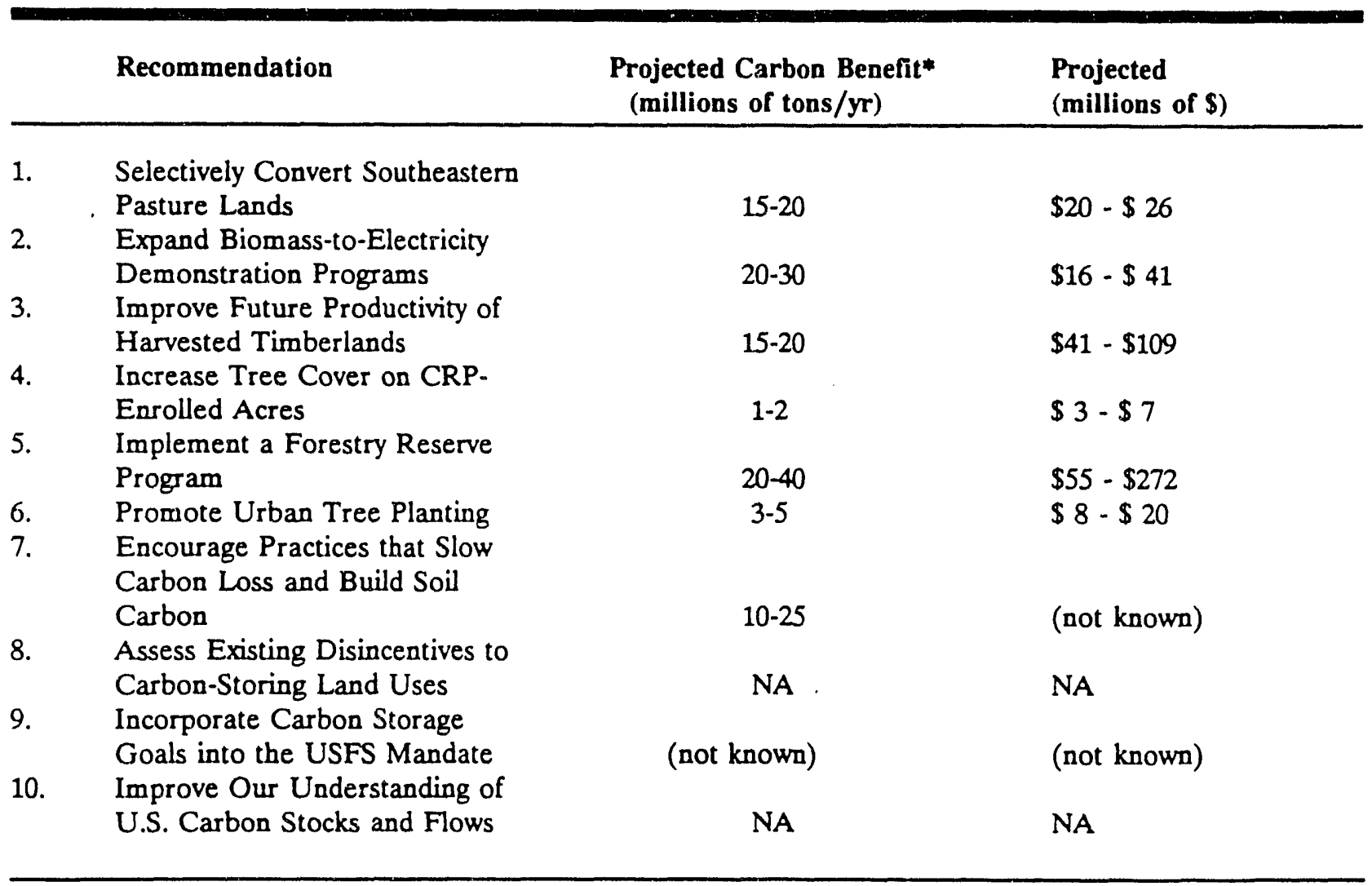

NA $=$ Not Applicable

- The carbon benefit figure estimates carbon storage in biomass and in soils as well as fossil fuel carbon displaced through implementation of the biotic options.

The Oregon Department of Energy estimated the cost of planting non-productive or unstocked timberland and replanting currently underproductive timberland: 
Planting

Unstocked Timberland $\quad \$ 21 /$ Ton to $\$ 39 /$ Ton, Avg $=\$ 26 /$ Ton

Replanting

Underproductive Timberland $\$ 35 /$ Ton to $\$ 66 /$ Ton, Avg $=\$ 44 /$ Ton

The average cost of these two options ( 0.55 million tons $\mathrm{CO}_{2}$ from the first and 1.06 million tons from the second) was about $\$ 40 /$ Ton of carbon dioxide taken up in forest growth.

\section{Solid Waste Management}

The cost of conventional landfill in the CONEG region ranges from $\$ 35 /$ Ton of solid waste in New Hampshire to about $\$ 110 /$ Ton in New York and New Jersey, with the other state's costs generally in between (e.g., \$70/Ton). Assuming the cost of collection is about $\$ 30 /$ Ton, landfill of solid waste costs about $\$ 65$ to $\$ 140 /$ Ton. The solid waste and greenhouse gas mitigation strategy options discussed earlier can benefit from off-setting this cost as well as reducing the other land-use and environmental impacts of landfill.

Urban solid waste recycling costs are about $\$ 30$ to $\$ 50 /$ Ton, depending on scale economies, with collection at about $\$ 60 /$ Ton. Thus, recycling, at $\$ 90-\$ 100 /$ Ton of solid waste, can have a marginal cost relative to landfill of plus or minus about $\$ 50 /$ Ton. Since, as shown earlier, recycling can eliminate about 0.43 tons of carbon equivalent (1.56 tons $\mathrm{CO}_{2}$ equivalent) from methane and carbon dioxide releases that would occur from one ton of solid waste at a landfill, the carbon dioxide reduction cost ranges from negative to $\$ 30 /$ Ton. Composting and MSW electric generating facilities can also achieve carbon reductions at negative or little net cost.

\subsection{Policy Recommendations}

\section{Establish Greenhouse Gas Goals at the State Level}

Much of the scientific work to date indicates that there is great potential for significant global climate change over the next several decades, from both past and likely future greenhouse gas releases, in the absence of major policy initiatives to reduce these emissions. Based on these findings, a variety of international and national organizations - e.g., the Intergovernmental Panel on Climate Change (IPCC) - have called for near and longer term targets for greenhouse gas emissions reductions, e.g. about 25 percent by 2005 and at least 50 percent by 2030 below current levels.

\footnotetext{
${ }^{39}$ Some have argued that "climate stabilization" will require at least $80 \%$ reductions from current levels.
} 
Following the scientific and policy work of the IPCC, an Intergovernmental Negotiating Committee (INC) was set up. INC will explore the promulgation of new protocols to limit worldwide and national releases of greenhouse gases. INC is also exploring mechanisms to ensure equitable allocation of responsibility and sharing of the financial burdens of such an ambitious undertaking, especially in light of the economic objectives of both the developing and industrial countries.

In the meantime, some governments have already taken unilateral action to begin the process of reducing greenhouse gas emissions (e.g., by both carbon taxes and reduction targets). Germany has a policy to reduce carbon dioxide levels 25 percent by 2005 and is investigating a $\mathrm{CO}_{2}$ tax of $\$ 6 /$ ton. Denmark has adopted a goal of 20 percent reduction for 2005 and is considering a tax of about $\$ 16 /$ ton. Various other European countries have carbon emissions stabilization or reduction policies. Sweden has enacted a carbon dioxide tax of about $\$ 40$ per ton.

The U.S. government has as yet made no commitment to either an international protocol or a national greenhouse gas reduction agenda. A bill has passed the Senate (S.324) calling for study of the implications of a 20 percent reduction in carbon emissions by 2005 , and one is before the House (HR 4805) calling for a $\$ 25 /$ ton tax on carbon ( $\$ 6.80 /$ ton carbon dioxide) for fossil fuels.

However, some U.S. states have take action. This can be seen as both a commitment to global climate stabilization and an economically sound approach to the investments that, it is believed, will inevitably come later, perhaps at significantly higher costs. The Oregon Department of Energy Plan (January 1991) recommends that Oregon establish emission targets for each greenhouse gas over the next 15 years. The proposed objective is a 20 percent reduction by 2005 . The Plan's policy is to promote the state's energy development consistent with a national carbon dioxide tax of $\$ 40$ per ton, and calls for such a national tax. ${ }^{40}$ In July 1991, a law requiring 20 percent reduction in greenhouse gas releases by 2000 was enacted by the Oregon legislature. The Vermont Department of Public Service's recent state energy plan targets a 12 percent reduction in $\mathrm{CO}_{2}$ by the year 2000. New York State has explored the costs and proposed protocols for realizing up to 10 percent reductions by 2010 .

Ambitious $\mathrm{CO}_{2}$ reduction strategies at the state level could assume a pro-rata share of the worldwide reductions that have been proposed, e.g., 25 percent by about 2005 and 50 percent by about 2030. Specific mechanisms could then be designed to meet these targets. Such goals, or more modest targets, might be established by a group of states, such as the CONEG states, as a matter of regional policy, until a more comprehensive national policy is developed. As noted above, i $i$ those states which have significant biomass resources, such policy targets could stimulate the development and

${ }^{40}$ It is noteworthy that Massachusetts and Nevada have adopted $\$ 25$ per ton $\mathrm{CO}_{2}$ adders for electric utility resource planning protocols, but not for other fuels as yet. Sweden has adopted a $\$ 40$ per ton $\mathrm{CO}_{2}$ tax, along with other pollutant taxes, to help send the correct price signals to all energy developers and users. 
use of such resources if they can be provided as a relatively low cost option in a sustainable and ecologically sound manner.

\section{Incorporate Environmental Costs into Planning}

The costs of environmental degradation are not included in traditional economic analysis for resource planning and investment decision. In energy, for example, environmental costs could be incorporated into many stages of the fuel cycle: extraction, processing, conversion, and delivery. This is already beginning in the regulated electric and gas utility sectors (within IRP rules, power supply bidding, and DSM bidding and evaluation). As noted earlier, some states in the region already have such protocols, although there is not a comprehensive and consistent system across all of the states.

In the CONEG region, Massachusetts, New York and New Jersey have adopted environmental externalities adders in their IRP, resource bidding, and/or DSM acquisition protocols. Vermont has adopted a 10 percent DSM credit and a 5 percent supply resource adder to reflect flexibility and societal costs inherent in these resource options. Other state utility regulators in the region have been actively considering adopting externalities costs in resource acquisition protocols. Recently, however, the Maine Public Utilities Commission rejected this course, citing the need for a more comprehensive national system. The $\mathrm{CO}_{2}$ value per ton is about $\$ 24$ in Massachusetts, about $\$ 14$ per ton in New Jersey, and about $\$ 2.20$ per ton - although much higher values are under discussion - in New York.

A national system covering all major pollutants including $\mathrm{CO}_{2}$ would be the most reasonable approach. This should be promoted by those organizations and agencies (such as CONEG) that support a climate stabilization policy. So too should a U.S. commitment to working out a comprehensive, efficient and fair international protocol. Until this occurs, state level initiatives could begin to make the resource selection process consistent with the longer term goals of environmental sustainability and climate stabilization in particular. The mechanisms that states could develop include sectorspecific regulations (as in electric utility IRP rules, transportation policies and investments) or all-sector all-fuels instruments (e.g., taxes or targets and trading).

At the very least, the momentum and experience already gathered in utility IRP regulation should be secured and extended throughout the states in the region and across the relevant power pools (NEPOOL, NYPP, and PJM). The use of a $\mathrm{CO}_{2}$ cost adder for electric and gas system planning and operating decisions should be encouraged throughout the CONEG region. Action at the power pool level could require initiatives at the Federal Energy Regulatory Commission, which regulates interstate wholesale transactions and pooling agreements.

While the use of a $\mathrm{CO}_{2}$ cost adder for resource planning would give appropriate credit to non-emitting resources, for biomass - unlike DSM, wind, geothermal, solar - it is necessary to close the carbon cycle by ensuring that enough is grown to off-set the carbon releases during combustion. The utility regulators should be encouraged to require sufficient, and ongoing, evidence that this is being carried out. Documentation 
of the character of the biomass resource, including the forest management practices used to provide it, could be required in the plans for the facility, just as in IRP and facility siting generally. Perhaps the state environmental and forestry agencies could provide the initial assessment and continued monitoring. A wood energy facility, including the fuel. source, that does not meet the requirement of a closed carbon cycle would not be acceptable." Beyond carbon balance itself, the environmental impacts of producing biomass crops or removing wood from the forests should be monitored and minimized by sound forestry practices, administered by the appropriate agencies.

Imposing a tax or ascribing a planning or resource acquisition cost adder for $\mathrm{CO}_{2}$ will cause non-emitting resources, such as sustainable produced biomass, to become more competitive with other options. Of course, other such non-emitting options, including DSM, solar, wind and geothermal will also become more competitive.

If, in addition to inclusion of environmental externalities costs, IRP and resource acquisition processes also gave recognition to fuel diversity, this would increase the role of renewable (and biomass) supplies. Then, the ultimate mix of resources that would likely comprise an IRP portfolio would depend upon availability, reliability, and the costs to construct and operate, along with the $\mathrm{CO}_{2}$ and other externalities cost adders. Biomass would likely find a place within such portfolios, depending upon the particular local conditions - natural, policy, land-use, and financial - for its development.

\section{Regulatory or Tax Incentives for Biomass and Other Renewables}

Incentives can be offered for the use of renewables. Federal tax incentives for solar energy have expired, so the burden may for the present lie with state governments. The inclusion of environmental externalities, as discussed above, may be sufficient to make biomass competitive, on an appropriate societal cost basis. However, incentives may be helpful during the early period of market development and commercialization.

In recent DSM discussions before utility commissions and elsewhere, utilities and other parties have proposed that special incentives be given to utilities - e.g., an extra return on investment, or a share of demonstrated resource savings - for successful implementation of DSM programs. Since this is a controversial policy - why pay more for the right resource choice - some support such an approach only as an interim measure to help shift utility managements' planning culture and expand utility experience with this relatively new service. A similar approach could be taken for renewable resources for a period of time, whether utility-developed or from independent power producers. To encourage utility investment in renewable energy technologies, state utility commissions could offer incentive rates of return for investing in renewable energy resources.

\footnotetext{
${ }^{41}$ A weaker version of this would ascribe the non-zero net carbon emissions cost to the facility.
} 


\section{Market Development}

Prior to the widespread use of concrete, steel, and other "modern" building materials, wood was used for the construction of bridges, structures, and other projects. The substitution of wood for other materials would reduce emissions from concrete (a major contributor to greenhouse gases) and would sequester carbon in long-lived structures. It could also provide the needed materials at a lower energy intensity.

One example of an application where wood could replace concrete and steel is the timber bridge. Made of local, low value hardwoods, stress-tested and pressure treated, it is an excellent substitute for other materials. It is readily usable in rural areas around the Northeast. The U.S. Forest Service has other examples of wood-based products that are relatively easy to start up. For these products to replace other materials, markets must be developed.

In the packaging industry, paperboard could displace other materials in certain instances where economical in terms of both direct and environmental life-cycle costs. This depends upon policies and practices affecting both the production of paperboard itself and the other materials (e.g., would energy- and emissions-intensive aluminum be from recycled or virgin sources). This area deserves careful study, and state-level market development policies may be warranted.

Market development could be promoted by the federal government through many established and functioning networks in the CONEG region, including research and demonstration projects, and educational/extension services. State agencies could also adopt policies that promote the use of wood materials where this is a more cost-effective and environmentally sound option over the long term, following study of the relative merits of the options available.

Government could also promote market development for other renewable energy products. Government facilities could purchase renewable energy technologies for their facilities (schools, offices, hospitals) in those cases where they are cost competitive and environmentally sound. For example, governments could purchase vehicles operating on biomass derived alcohol as a means to stimulate this market. Fleet vehicles could provide the basis for economies of scale. Here again, overall economics, environmental characteristics, and the merits of diversity might oblige agencies to compare and perhaps mix a variety of promising options such as natural gas, electricity, diesel and biofuels.

\section{Forest Management Education}

The forests of the Northeast are not as productive they could be. Forest management is limited in the region, primarily because it is uneconomical. Increasing the demand for wood, such as might happen if the Vermont's Bennington project comes on line, could lead to price increases that would encourage better forest management. Such projects might also encourage management by providing a long-term stable market for low value hardwoods and could also encourage landowners to take a longer term view of their resource and its management. 
There is a potential audience of some 350,000 owners of forest land in the CONEG states that could be further educated concerning modern, environmentallyconscious forestry management practices. To do this it is necessary to develop educational approaches that effectively communicate both the benefits to the atmosphere which come from better managed forests and the value of the wood products which are harvested from those forests. There are several programs, both public and private, through which such an effort could be pursued.

The American Tree Farm Program is an industry sponsored effort to recognize and encourage owners of 10 or more acres of timberland who prepare and adhere to management plans for their timberland. Tree farms are certified by forestry experts in each of the 50 states. Massachusetts, Connecticut, and Rhode Island have had landowners involved in the Tree Farm Program since the late 1940s and mid 1950s. Today there are 2,015 tree farms in those three states, representing about 8 percent of the private timberland acreage.

Both the American Tree Program and the USFS State and Private Forestry Program are well established networks to reach and motivate the small, non-industrial private owners. CONEG initiatives or strategies to influence the take-up of $\mathrm{CO}_{2}$ by trees should work closely with these well-established institutions. The Forest Stewardship Act of 1990, within the Food, Agriculture, Conservation, and Trade Act of 1990, outlines many of the goals and means the USFS has to work with private owners through, and in cooperation with, the individual state foresters. The Act recognizes that nearly half of the U.S. wood supply comes from private lands, these lands can benefit from better forest management, and the pressures to develop these lands for non-timber uses is escalating. The Act authorizes the Secretary of Agriculture to, engage in, among other things, "...strengthening educational, technical, and financial assistance programs that provide assistance to owners of non-federal forest lands..."

On the school front, additional educational materials can be developed. These might include enhancements to "Project Learning Tree", the American Forest Council's teachers' curriculum guide for trees and forestry, which makes schoolchildren aware of the tremendous energy values derived from trees and forests - for clean air, shading, windbreaks, and energy efficient products.

\section{Short Rotation Crops}

Biomass in general, and forest resources in particular, have been used on a relatively small scale in the U.S., and elsewhere, to produce energy, including electric power and transportation fuels. Some of the CONEG states have promoted biomass-toenergy applications, typically using agricultural or forest residues. However, if the larger potential for this resource is to be realized, especially in the electric power and transportation sectors, it will be important to research and experiment with new technologies and techniques. Some important dimensions of the process are:

- $\quad$ improving the efficiency of biomass-to-energy conversion facilities;

- $\quad$ reducing the pollutant emissions from these facilities; 
- $\quad$ keeping the costs and operating characteristics of such facilities competitive with the alternatives;

- developing effective means to produce large amounts of biofuel feedstocks through short rotation woody and herbaceous crops; and

- developing the knowledge and operating principles to ensure that largescale biomass resources are produced in a an ecologically sound manner.

All of these elements would help to ensure that economies in cost and land-use, and protection of air, water and environmental resources are realized.

There are currently no short rotation crop plantations in the Northeast. Demonstration projects should be encouraged to test the viability and environmental consequences of these projects in specific locations throughout the CONEG states. Land-use patterns, climate, rainfall, soil conditions, existing vegetation, and competing commercial opporturities may all need to be studied in order to screen and prioritize the locations, species, infrastructure and other requirements for economical biomass energy crops. State energy, environmental, agricultural, and forestry agencies, with support from industry and research organizations, could develop research programs to address these factors.

These projects should be tested to determine the option's long term technological, environmental, and economic feasibility. Demonstration efforts need to be directed not only at producing biomass more efficiently but also at converting it more efficiently to electricity. The ongoing testing of BIG STIGs at General Electric Company in Schenectady, New York is an example of this. (Currently, conventional wood power plants have heat rates of from 14,000 to $20,000 \mathrm{Btu} / \mathrm{kwh}$, while BIG STIGS promise about 10,000 .) The states in the CONEG region have strong industrial, engineering, research and educational, and governmental institutions, that could vigorously promote the research and development of environmentally sound biomass technologies.

\section{Urban Trees}

As discussed earlier an ambitious program of urban tree planting can have multiple benefits. By shading and other mechanisms strategically planted urban trees can reduce residential and commercial space conditioning energy requirements. They can also take up carbon from the atmosphere and store it indefinitely if well maintained. Urban trees can provide aesthetic amenities, in neighborhoods, in parks, along commuter highways, and in greenbelts. It can also help in the retention of scarce urban soils. As estimated by WRI urban tree planting can be realized at relatively low costs per ton of carbon dioxide sequestered.

\section{Solid Waste Management Policies}

Degradable carbon in landfill, which derives from biomass, contributes both $\mathrm{CO}_{2}$ and methane to the atmosphere. Policies to reduce these emissions, while perhaps achieving energy and economic benefits, make sense in the current context. The scarcity 
of landfill space in the region, and the potentially harmful emissions of toxics that could derive from incineration, have encouraged analysis and policy-makers to seek nther methods for solid waste management, including waste-to-energy facilities for methane capture or combustion, recycling, and composting of organic waste.

Combustion of municipal solid waste contributes essentially all of the carbon content to the atmosphere in the form of $\mathrm{CO}_{2}$, while the anaerobic decomposition of organics in landfill emits carbon in carbon dioxide and carbon in methane in equal amounts. Even if the organics material were produced sustainably (with growth equal to use and release) in a closed carbon cycle, from a global warming perspective the landfill releases would contribute to net warming owing to the higher global warming potential of carbon in methane. Thus, solid waste combustion, especially if it displaces fossil combustion, is a promising option in this regard. Unfortunately, there is understandable concern about the emissions of toxics from the stacks of such facilities and in the waste products that require disposal. This, along with local siting constraints and opposition, will likely limit the amount of combustion that will ultimately take place.

As a consequence of such concerns, re-cycling and composting, both of which turn some of the waste into useful products, have been on the policy agendas of environmental and state agencies. The first limits the volume of waste and the second produces carbon dioxide and not methane (and thus, no net carbon emissions in a sustainable cycle). Beyond these options, source reduction strategies and policies at the state level could reduce the overall levels of wastes, and perhaps their toxicity as well, entering the solid waste management nexus.

Even if ambitious policies for source reduction, re-cycling and composting are adop:ed at the state level, there will likely remain some residual waste that might need to be either landfilled $n$ r burned. Preferably, policies would be adopted to ensure that their use for energy be encouraged where cost-effective compared with alternatives and that stringent requirements on residual pollutants and appropriate siting be enforced.

\section{Environmental Management}

Threats to the environment from biomass projects should not be underestimated. Large scale biomass projects will disrupt the natural environment. Monocultures of fast growing trees can reduce soil nutrients, the diversity of vegetation, and species diversity. These projects may also increase road access to the forest which could also be disruptive of the natural environment. A final unknown concerning biomass is genetic engineering. If new species are introduced into the natural environment it is not known how they will int ract with this environment. Many experiments of this sort have gone awry, the creation and migration of the "killer" bees being only among the most recent. Similar concerns have jeen voiced about genetically engineered farm crops.

Despite the environmental threats from large scale biomass production, the threat to the environment from global warming is equally if not more serious. For this reason, environmentalists have reason to work with biomass energy developers to develop technology and guidelines for use that are mutually acceptabie (Beyea et al, 1991). 
The Audubon Society is hopeful that cooperation to protect bio-diversity will occur. Keeler and Beyea write:

Without recognition of and a change in emphasis on environmental quality, genetic engineering for the production of energy and raw materials will likely take place independent of providing environmental protection. It would be much wiser and cheaper in the long run to better integrate environmental concerns into energy biotechnology planning from the beginning. Because the technology is new, the opportunity exists to channel biomass engineering into environmentally sound directions, integrating environmental safeguards into the product design process. 


\section{References}

The American Forestry Association. 1989 Street Tree Survey of U.S. Cities and Towns. The American Forestry Association, Washington, DC, July, 1990.

Analytical and Economic Challenges in Assessing $\mathrm{CO}_{2}$ Abatement Costs and Policies, 1990. Proceedings of the Workshop on Economic/Energy/Environmental Modeling for Climate Policy Analysis. David O. Wood and Tioichi Kaya, eds. Washington, D.C. October, p. 549.

Air and Energy Engineering Research Laboratory, 1989. The 1985 NAPAP Emissions Inventory (Version 2): Development of the National Utility Reference File. Prepared for the National Acid Precipitation Assessment Program, EPA-600/7-89-013a, November.

Akbari, H. Huang, J. Matien, et al., 1989. Saving Energy and Reducing Atmospheric Pollution by Controlling Summer Heat Islands, in Controlling Summer Heat Islands: Proceedings of the Workshop on Saving Energy and Reducing Atmospheric Pollution by Controlling Summer Heat Islands, LBL-27872, Berkeley, CA, 1989.

Akbari, H., 1991. Personal Communication.

Anderson, Dennis, 1991. The Forestry Industry and the Greenhouse Effect. Scottish Forestry Trust.

Beyea, Jan, J. Cook and Kathleen Keeler, 1991. Potential Impacts of Biomass Production in the United States on Biological Diversity, April 23, p 5.

Beyea, Jan and Kathleen Keeler, 1991. Biotechnological Advances in Biomass Energy and Chemical Production: Impacts on Wildlife and Habitat. Critical Reviews of Biotechnology, February.

The Carbon Dioxide Information Analysis Center, Oak Ridge National Laboratory, 1990. A Compendium of Data on Global Change, Oak Ridge Tennessee, August.

Carver, Phil, no date. SB 576 Global Warming Emissions Reduction Strategies, Technical Appendices. Oregon Department of Energy.

The Climate Institute, 1989. Coping with Climate Change. Proceedings of the Second North American Conference on Preparing for Climate Change: A Cooperative Approach, Washington, D.C. June.

Considine, Thomas, 1984. An analysis of New York's Timber Resources. USFS: pp. 46 and 50. 
Cook, James and Jan Beyea, 1991. Potential Impacts of Biomass Production in the United States on Biological Diversity, April, pp. 28.

Cook, James H, 1991. Preserving Biological Diversity in the Face of Large-Scale Demands for Biofuels. Energy from Biomass and Wastes, XV, Washington, DC, March 25-29.

Cook, Jim, 1991. Toward Ecological Guidelines for Large-Scale Biomass Energy Development. National Audubon Society. July 1, draft.

Cost Effectiveness Analysis of $\mathrm{CO}_{2}$ Reduction Options: Country Reports, 1991. Report for the Commission of the European Communities Directorate General XII. May.

The Economist, 1991. The Future of the Forests. June 22, pp. 19-23.

Electric Power Research Institute, 1990. Wood - America's Renewable Fuel. EPRI Technical Brief RP2612-12, Palo Alto, CA.

European Environmental Bureau, 1989. Energy Policy in the Greenhouse. Volume 1. From Warming Fate to Warming Limit. International Project for Sustainable Energy Paths, September.

Glenn, J. and D. Riggle, 1991. The State of Garbage in America. Biocycle, May.

Grubb, M.J., 1990. Analytical and Economic Challenges in Assessing CO, Abatement Costs and Policies,Proceedings of the Workshop on Economic/Energy/Environmental Modeling for Climate Policy Analysis, Washington, DC. October, p. 547.

Hubbard, James. E., 1989. Private Nonindustrial Forests. American Forests. November/December, pp. 24.

ICF Resources Incorporated, 1990. Preliminary Technology Cost Estimates of Measures Available to Reduce US Greenhouse Gas Emissions by 2010. Submitted to: US EPA, August.

Idaho National Engineering Laboratory, Los Alamos National Laboratory, et al., 1990. The Potential of Renewable Energy. An Interlaboratory White Paper, March.

Intergovernmental Panel on Climate Change, 1990. Potential Impacts of Climate Change, Repnrt from Working Group II, August.

Larson, E.D and R.H. Williams, 1990. Biomass-Gasifier Steam-Injected Gas Turbine Cogeneration. Journal of Engineering for Gas Turbines and Power. April, Vol. 112, pp. 157-163. 
Maine Department of Conservation, 1988. Forest for the Future, a Report on Maine's Forest to the Legislature, the Governor, and the People of Maine. Maine Department of Conservation, Augusta, ME, January, p. 3.

Manne, Alan S. and Richard G. Richels, 1989. $\mathrm{CO}_{2}$ Emission Limits: An Economic Cost Analysis for the U.S.A. Electric Power Research Institute. Palo Alto, California, November.

Marland, Gregg, 1989. Reforestation: Pulling Greenhouse Gases Out of Thin Air. Biologue and the Regional Biomass Energy Program Reports. April/May, pp. 14-18.

Meyer, Alden, et al., 1991. America's Energy Choices: Investing in a Strong Economy and a Clean Environment. A joint project of Alliance to Save Energy, American Council for an Energy Efficient Economy, Natural Resources Defense Council, Union of Concerned Scientists, in consultation with Tellus Institute. Union of Concerned Scientists, Cambridge, Massachusetts, September.

Mills, Evan, Deborah Wilson and Thomas B. Johansson, 1991. Getting Started: Noregrets Strategies for Reducing Greenhouse Gas Emissions. Energy Policy, July/August.

Moulton, Robert J. and Kenneth R. Richards, 1990. Costs of Sequestering Carbon through Tree Planting and Forest Management in the United States. August 27 (draft).

National and State Level Emissions Estimates of Radiatively Important Trace Gases From Anthropogenic Sources. U.S. EPA. PB91-103572. Washington, D.C. October 1990.

New York State Energy Office, 1991. Analysis of Carbon Reduction in New York State, Albany, N.Y. June.

Nordhaus, William D., 1990. "To Slow or Not to Slow: The Economics of the Greenhouse Effect." The Economic Journal, 101. July, pp. 920-937.

North American Electric Reliability Council. 1989 Electricity Supply \& Demand for 19891998, Annual Data Summary. North American Electric Reliability Council, Princeton, NJ, October, 1989.

Office of Technology Assessment, 1991. Energy Technology Choices: Shaping Our Future, Washington, D.C, July.

Office of Technology Assessment. No date. Energy From Biological Processes, Technical and Environmental Analyses, Ballinger: Cambridge, pp. 12-13.

Oregon Department of Energy. Fourth Biennial Energy Plan. State of Oregon, January 1991. 
Oregon Department of Energy, 1990. Oregon Task Force on Global Warming Report to the Governor and Legislature, Salem, Oregon, June.

Oregon Department of Energy, 1991. Fourth Biennial Energy Plan, Salem, Oregon, June.

Perlin, John. 1989. A Forest Journey, The role of wood in the development of Civilization, New York: Norton, page 360.

Peterson, Jeffrey M. Wood Energy Technology Assessment. New York State Energy Research and Development Authority, July 1991.

Piccot, Stephen and Mark Saeger. National and State Level Emissions Estimates of Radiatively Important Trace Gases (RITGs) from Anthropogenic Sources. US Department of Commerce, October, 1990.

Rader Nancy, K. Bossong, Jonathan Becker, Daniel Borson, and Cleo Manuel. 1990. The Power of the States: A Fifty State Survey of Renewable Energy. Public Citizen Critical Mass Energy Porject, Washington, D.C., June.

Rader, Nancy, Ken Bossong et al. The Power of the States, Supplement: State-by-State Data Sheets. Public Citizen Critical Mass Energy Project, Washington, DC, June 1990.

Rosenberg, Norman J., et al., 1989. Greenhouse Gas Warming: Abatement and Adaptation, Resources for the Future. Washington, D.C.

Seymour, Robert. 1991. Personal communication, February 12.

Seymour, Robert. "Productivity of Maine's Forest Underestimated -- More intensive Approaches are needed", page 209.

Seymour, Robert and Ronald Lemin. 1989. "Timber Supply Projections for Maine, 1980-2080." CFRU Research Bulletin 7, February, pp. 6 and 32.

Shands, William. E. "Federal Forests." American Forests. November/December, 1989, p. $22+$.

Stone, B. 1991. Personal Communication. Vermont Department of Forests, Parks and Recreation.

Strauss, Charles and Lynn Wright. Woody Biomass Production Costs in the United States: An Economic Summary of Commercial Populus Plantation Systems, Solar Energy, Vol 45, No. 2, pp. 105-110.

Tellus Institute, 1991. Greenhouse Gas Scenario System. Stockholm Environment Institute -- Boston Center, Boston, MA. 
Tellus Institute. Existing and Future Solid Waste Management Systems in the RPA Region. Tellus Institute, Boston, MA, October 8, 1991.

Tellus Institute, 1987. An Evaluation of Central Maine Power Company's Proposed Purchase of Power from Hydro Quebec, Report No. 87-30, Tellus Institute, Boston, Massachusetts.

Trexler, Mark C. Minding the Carbon Store: Weighing US Forestry Strategies to Slow Global Warming. World Resources Institute, Washington, DC, January 1991.

United States Department of Agriculture, Forest Service. Aboveground Tree Biomass Statistics for Maine: 1982. Resource Bulletin NE-86.

United States Department of Agriculture, Forest Service. Forest Statistics for New Hampshire - 1973 and 1983. Resource Bulletin NE-88.

United States Department of Agriculture, Forest Service. Forest Statistics for Massachusetts - 1972 and 1985. Resource Bulletin NE-106.

United States Department of Agriculture, Forest Service. Forest Statistics for New Jersey 1987. Resource Bulletin NE-112.

United States Department of Agriculture, Forest Service. Forest Statistics for New York 1980. Resource Bulletin NE-71.

United States Department of Agriculture, Forest Service. Forest Statistics for Pennsylvania - 1978. Resource Bulletin NE-65.

United States Department of Agriculture, Forest Service. Forest Statistics for Maryland 1976 and 1986. Resource Bulletin NE-107.

United States Department of Agriculture, Forest Service. Forest Statistics for Maine 1971 and 1982. Resource Bulletin NE-81.

United States Department of Agriculture, Forest Service. Biomass Statistics for New Hampshire - 1983. Resource Bulletin NE-92.

United States Department of Agriculture, Forest Service. Biomass Statistics for Vermont 1983. Resource Bulletin NE-91.

United States Department of Agriculture, Forest Service. Forest Statistics for Connecticut - 1972 and 1985. Resource Bulletin NE-105.

United States Department of Agriculture, Forest Service. Forest Statistics for Delaware 1972 and 1986. Resource Bulletin NE-109. 
United States Department of Agriculture, Forest Service. Resource Bulleting NE-87. Forest Statistics for Vermont, p. 14.

United States Department of Agriculture, Forest Service. Forest Statistics for Rhode Island - 1972 and 1985. Resource Bulletin NE-104.

United States Department of Agriculture, Forest Service. 1990b. FY 1989 U.S. Forest Planting Report, March, Table 6.

United States Department of Agriculture, Forest Service. Research Paper NC-268. Factors and Equations to Estimate Forest Biomass in the North Central Region, by W. Brad Smith. Writes that $26 \%$ of the tree is in the stump and top, p. 3.

United States Department of Agriculture, Forest Service. 1987. The Economic Importance of Vermont's Sawtimber, by J. Michaels, et al. Northeastern Forest Experiment Station, NE-RP-587, September.

United States Department of Agriculture, Forest Service. 1987. The Timber Industries of Southern New England, A Periodic Assessment of Timber Output, p. 14.

United States Department of Agriculture, Forest Service. 1990. Northern Forest Lands Study, April, p. 9.

United States Department of Agriculture, Forest Service. 1990a. "Developing Markets for Low Quality Wood: Wood Supply in Bennington County and Surrounding Area." December, p. 23.

U.S. Department of Energy. Regional Reports for the Annual Energy Outlook 1990. US Department of Energy, Washington, DC, April 26, 1990.

U.S. Environmental Protection Agency, 1990. Policy Options for Stabilizing Global Climate, Report to Congress, Washington, D.C., December.

U.S. Environmental Protection Agency. National Emissions Data System Information (First Edition). EPA-450/4-80-013a, US EPA Office of Air and Radiation, Research Triangle Park, NC, November 1984.

Walt, Harold R. "Industrial Forests." American Forests. November/December, 1989, p. $26+$.

Wilcove, David S. "Protecting Biodiversity in Multiple-use Lands: Lessons from the US Forest Service." Trends in Ecology and Evolution. December 1989, Vol. 4, No. 12.

Williams, Robert H. Biomass Gasifier/Gas Turbine Power and the Greenhouse Warming. IEA/OECD Expert Seminar on Energy Technologies for Reducing Emissions of Greenhouse Gases, Paris, April 12-14, 1989. 
Wisconsin Department of Natural Resources, 1991. Global Climate Change Strategies for Wisconsin, Madison, Wisconsin, October.

World Resources Institute, "Slowing Global Warming Biotically - Options for the United States" in Minding the Carbon Store: Weighing U.S. Forestry Strategies to Slow Global Warming, WRI, 1991.

Young, Harold. 1982. Principles of Complete Forest Management Applied to the West Half of T4-R16, Elm Stream Township, Maine, Orono: University of Maine, p. 25. 
APPENDIX A

CONEG REGION SOLID WASTE SYSTEM:

STATE-SPECIFIC GREENHOUSE GAS EMISSIONS 
The following sets of tables provide state-specific and CONEG region-wide greenhouse gas emissions over the period 1988 to 2020 for a Reference Case and Policy Case, respectively. Each of these cases begins with a region-wide summary table, followed by the more detailed state-by-state tables upon which the summaries are based. The basic assumptions regarding the magnitude of the residential and commercial solid waste stream, the percentage of that stream diverted by source reduction (SR), recycling, and composting, and the amounts designated for MSW incineration and landfill gas (methane) resource recovery are given for each state.' These policy related assumptions are changed over time and between scenarios, reflecting plausible assumptions about the impact of current plans (Reference Case) and more ambitious strategies (Policy Case).

Emissions of both carbon dioxide $\left(\mathrm{CO}_{2}\right)$ and methane $\left(\mathrm{CH}_{4}\right)$ are estimated for all sources. These include: "landfill emissions" of both $\mathrm{CO}_{2}$ and $\mathrm{CH}_{4}$ released from the degradation of organic waste in landfill (not including the gas captured for energy recovery); "compost emissions" of $\mathrm{CO}_{2}$ from aerobic decomposition of carbonaceous material in the production of compost; and $\mathrm{CO}_{2}$ "emissions from burning" in MSW and captured landfill gas energy facilities. In addition, it is assumed that all of the organic carbon in the waste stream has been produced through the uptake of atmospheric carbon, and thus "carbon uptake" is subtracted from total carbon emissions to give net emissions. The global warming potential of methane is taken to be a factor of 10 times that of carbon dioxide and thus the carbon equivalents of $\mathrm{CH}_{4}$ and $\mathrm{CO}_{2}$ are:

$$
\begin{array}{ll}
\mathrm{CH}_{4} \mathrm{C} \text { Equiv: } & 10 \times 12 / 44=2.73 \\
\mathrm{CO}_{2} \mathrm{C} \text { Equiv. } & 12 / 44=0.27
\end{array}
$$

Other assumptions embodied in the following tables are:

$\begin{array}{lll}\begin{array}{l}\text { Fraction of waste (by weight) as carbon } \\ \text { Fraction of carbon (by weight) degraded }\end{array} & = & 0.23 \\ \begin{array}{l}\text { Fraction of degraded carbon (by volume) } \\ \text { released as } \mathrm{CO}_{2}\end{array} & =0.8 \\ \begin{array}{l}\text { Fraction of degraded carbon (by volume) } \\ \text { released as } \mathrm{CH}_{4}\end{array} & =0.5 \\ & =9 \mathrm{MMBtu} / \mathrm{Ton} \\ \begin{array}{l}\text { Energy content of waste } \\ \mathrm{CO}_{2} \text { emissions from waste combustion }\end{array} & =165 \mathrm{lb} / \mathrm{MMBtu}\end{array}$

1 A source reduction initiative adopted by CONEG in 1991 called for reduction in packaging waste to a level that contributes to the overall goal of at least 50 percent reduction in all solid waste in the Northeast by the year 2000. As a result, in 1992 the CONEG Source Reduction Task Force drafted model legislation that sets a 15 percent packaging reduction standard for 1996 and a 35 percent packaging reduction goal for the year 2000. Waste from packaging currently makes up about one-third of the municipal solid waste stream. Although the CONEG Source Reduction Initiative is indicative of the kind of plans and policies anticipated in this discussion, the source reduction projections used by Tellus to build the Reference Case and other scenarios were developed prior to adoption of this policy by the CONEG states, and do not correspond to the reduction targets set by this policy. 
New England

\section{CONEG Region Solid Waste System \\ Greenhouse Gas Emissions}

(Million Tons)

Reference Case

$\begin{array}{lllll}1988 & 1996 & 2004 & 2012 & 2020\end{array}$

Landfill Emissions

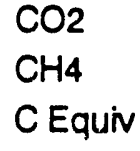

\begin{tabular}{rrrrr}
1.83 & 1.64 & 1.68 & 2.01 & 2.29 \\
0.75 & 0.42 & 0.12 & 0.18 & 0.24 \\
\hline 2.14 & 1.37 & 0.73 & 0.93 & 1.14
\end{tabular}

Res Recovery Emissions

$\mathrm{CO} 2$

$C$ Equiv

\begin{tabular}{lllll}
4.65 & 5.67 & 5.93 & 5.93 & 5.93 \\
\hline 1.27 & 1.55 & 1.62 & 1.62 & 1.62 \\
3.41 & 2.91 & 2.34 & 2.55 & 2.76
\end{tabular}

Mid Atlantic

Landfill Emissions

$\mathrm{CO} 2$
$\mathrm{CH} 4$
$\mathrm{C}$ Equiv

\begin{tabular}{lllll}
6.53 & 4.19 & 3.79 & 3.73 & 3.78 \\
2.20 & 1.25 & 1.05 & 0.97 & 0.96 \\
\hline 7.79 & 4.54 & 3.90 & 3.68 & 3.64
\end{tabular}

Res Recovery Emissions

$\mathrm{CO} 2$
$\mathrm{C}$ Equiv

Total C Equivalent

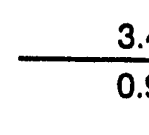

8.73

3.44
6.87

1.87

6.41

5.77

5.55

5.51

New York

Landfill Emissions
$\mathrm{CO} 2$
$\mathrm{CH} 4$
C Equiv

\begin{tabular}{rrrrr}
4.71 & 3.61 & 3.27 & 2.82 & 3.19 \\
1.71 & 1.31 & 1.19 & 1.03 & 1.16 \\
\hline 5.96 & 4.56 & 4.14 & 3.57 & 4.03
\end{tabular}

Res Recovèry Emissions

$\mathrm{CO} 2$

- C Equir

\begin{tabular}{lllll}
2.95 & 3.40 & 3.40 & 3.40 & 3.40 \\
\hline 0.80 & 0.93 & 0.93 & 0.93 & 0.93
\end{tabular}

Total C Equivalent

6.76

5.49

5.06

4.49

4.96

CONEG Region

Landfill Emissions

\begin{tabular}{lrrrrr}
$\mathrm{CO} 2$ & 13.07 & 9.44 & 8.74 & 8.56 & 9.25 \\
$\mathrm{CH} 4$. & 4.67 & 2.97 & 2.35 & 2.18 & 2.35 \\
\cline { 2 - 6 } $\mathrm{C}$ Equiv & 15.89 & 10.47 & 8.76 & 8.17 & 8.81
\end{tabular}

Res Recovery Emissions

$\mathrm{CO} 2$

C Equin

\begin{tabular}{rrrrr}
11.03 & 15.94 & 16.20 & 16.20 & 16.20 \\
\hline 3.01 & 4.35 & 4.42 & 4.42 & 4.42
\end{tabular}




\begin{tabular}{rrrrr}
1988 & 1996 & 2004 & 2012 & 2020 \\
\hline 20.26 & 21.94 & 23.94 & 26.07 & 28.24 \\
$0 \%$ & $10 \%$ & $10 \%$ & $10 \%$ & $10 \%$ \\
$11 \%$ & $20 \%$ & $30 \%$ & $40 \%$ & $40 \%$ \\
$0 \%$ & $0 \%$ & $0 \%$ & $0 \%$ & $0 \%$ \\
3.50 & 4.10 & 4.10 & 4.10 & 4.10 \\
14.54 & 11.26 & 10.27 & 8.94 & 10.02 \\
0.57 & 0.57 & 0.57 & 0.57 & 0.57 \\
& & & & \\
13.97 & 10.69 & 9.70 & 8.37 & 9.45
\end{tabular}

Landfill Emissions

$\mathrm{CH} 4$ fromLF

As $C$ equivalent

$\mathrm{CO} 2$ from LF

As C

Compost Emissions

$\mathrm{CO} 2$ from Compost

As $\mathrm{C}$

$\mathrm{CO} 2$

C Tons ${ }^{\wedge} 6$
0.00

0.00

1.71

4.67

4.71

1.28

1.31

3.58

3.61

0.98

0.00

0.00

\section{Emissions from Burning}

MSW RR

As $\mathrm{C}$

Landfill Gas

As $\mathrm{C}$

Total Emissions

As Carbon Equivalent
CO2 Tons^ 6

$C$ Tons ${ }^{\wedge} 6$

CO2 Tons ${ }^{\wedge} 6$

$C$ Tons $^{\wedge} 6$

$C$ Tons ${ }^{\wedge}$

6.76

\section{Carbon Uptake}

As Carbon Equivalent

C Tons ${ }^{\wedge} 6$

3.73

Net Emissions

As Carbon Equivalent
C Tons $₹$

3.03
1.19

3.24

3.27

0.89

0.00

0.00
1.03

2.80

2.82

0.77

0.00

0.00

0.00

0.00

1.16

3.16

3.19

0.87
5.49

5.06

4.49

4.96

4.04

4.41

4.80

5.20

$\begin{array}{lll}1.45 & 0.66 & -0.30\end{array}$

$\begin{array}{lll}1.45 & 0.66 & -0.30\end{array}$

3.06

3.06

0.84

0.33

0.09

\begin{tabular}{ll}
0.09 & 0.09 \\
\hline
\end{tabular}

0.09

0.33

$-0.24$ 
Total Waste

SR

Recycling

Compost

Resource Recovery

Net Landfill

LF Methane Recovery

Degrading in the Landfill

$10^{\sim} 6$ tons

\%SR.

\%RC

\%Compost

$10^{\wedge} 6$ tons

$10^{\wedge} 6$ tons

$10 \Upsilon 6$ tons

$\mathrm{CH}_{4}$ Tons $^{\wedge} 6$

$C$ Tons $₹$

$\mathrm{CO} 2$ Tons $^{\wedge} 6$

$\mathrm{CO} 2$

C Tons ${ }^{\circ}$

0.04

0.01

0.06

0.02

0.10

0.16

0.04

0.17

0.03

0.05

$0 \%$

$10 \%$

$20 \%$

0.61

0.28

0.06

0.22

0.03

0.07

0.07

0.02

\section{Emissions from Burning}

MSW RR

As $\mathrm{C}$

Landfill Gas

As $\mathrm{C}$

\section{Total Emissions}

As Carbon Equivalent

\section{Carbon Uptake}

As Carbon Equivalent

C Tons 76

0.14

0.16

0.19

0.21

0.23

Net Emissions

As Carbon Equivalent

0.46

0.12

0.46

0.12

0.04

0.46

0.46

0.46

0.04

0.04

0.12

0.12

CO2 Tons

0.01

0.01

0.01

0.04

0.01

C Tons $\gamma$

0.15

0.19

0.22

0.24

0.27

$c$ Tons $ซ 6$

0.01

0.02

0.03

0.02

0.04

0.01


Total Waste

SR

Recycling

Resource Recovery

LF Methane Recovery
Compost

Net Landfill $10^{\sim} 6$ tons

\%SR

\%RC

\%Compost

$10^{\wedge} 6$ tons

$10^{\Upsilon} 6$ tons

$10^{\wedge} 6$ tons
5.47

$0 \%$

$0 \%$

$0 \%$

1.09

4.38

0.16

4.22

3.34

3.79

4.13

4.60

Landfill Emissions

$\mathrm{CH} 4$ fromLF

As $C$ equivalent

$\mathrm{CO} 2$ from $L F$

As $\mathrm{C}$

\section{Compost Emissions}

$\mathrm{CO} 2$ from Compost

As $\mathrm{C}$
$\mathrm{CH}_{4}$ Tons $^{\wedge} 6$

C Tons ${ }^{\top} 6$

CO2 Tons 6
0.52

1.41

1.42

0.39

$\mathrm{CO} 2$

$c$ Tons $₹$
0.00

0.00
0.41

1.12

1.13

0.31

0.00

0.00
0.47

1.27

1.28

0.35

0.00

0.00
6.73

$0 \%$

$20 \%$

$0 \%$

1.09

4.29

0.16

4.76

0.16

$0 \%$

$20 \%$

$0 \%$

1.09
7.32

16

0.56

1.54

1.55

0.42

0.38

0.00

0.00

0.00

0.00

\section{Emissions from Burning}

MSW RR

As $\mathrm{C}$

Landfill Gas

As $\mathrm{C}$
CO2 Tons ${ }^{\wedge} 6$

$C$ Tons ^ 6

CO2 Tons ${ }^{\wedge} 6$

$C$ Tons $\mathcal{} 6$
0.81

0.22

0.09

0.03

2.05

1.67

1.87

2.01

As Carbon Equivalent $C$ Tons $` c$

\section{Carbon Uptake}

As Carbon Equivalen

C Tons $\dot{6}$

1.01

0.94

1.09

1.24

Net Emissions

As Carbon Equivalent $\quad C$ Tons $\mathcal{6}$
1.04

0.73

0.77

0.77

0.87
2.21

1.35

0.81

0.22

0.09

0.03

0.03 


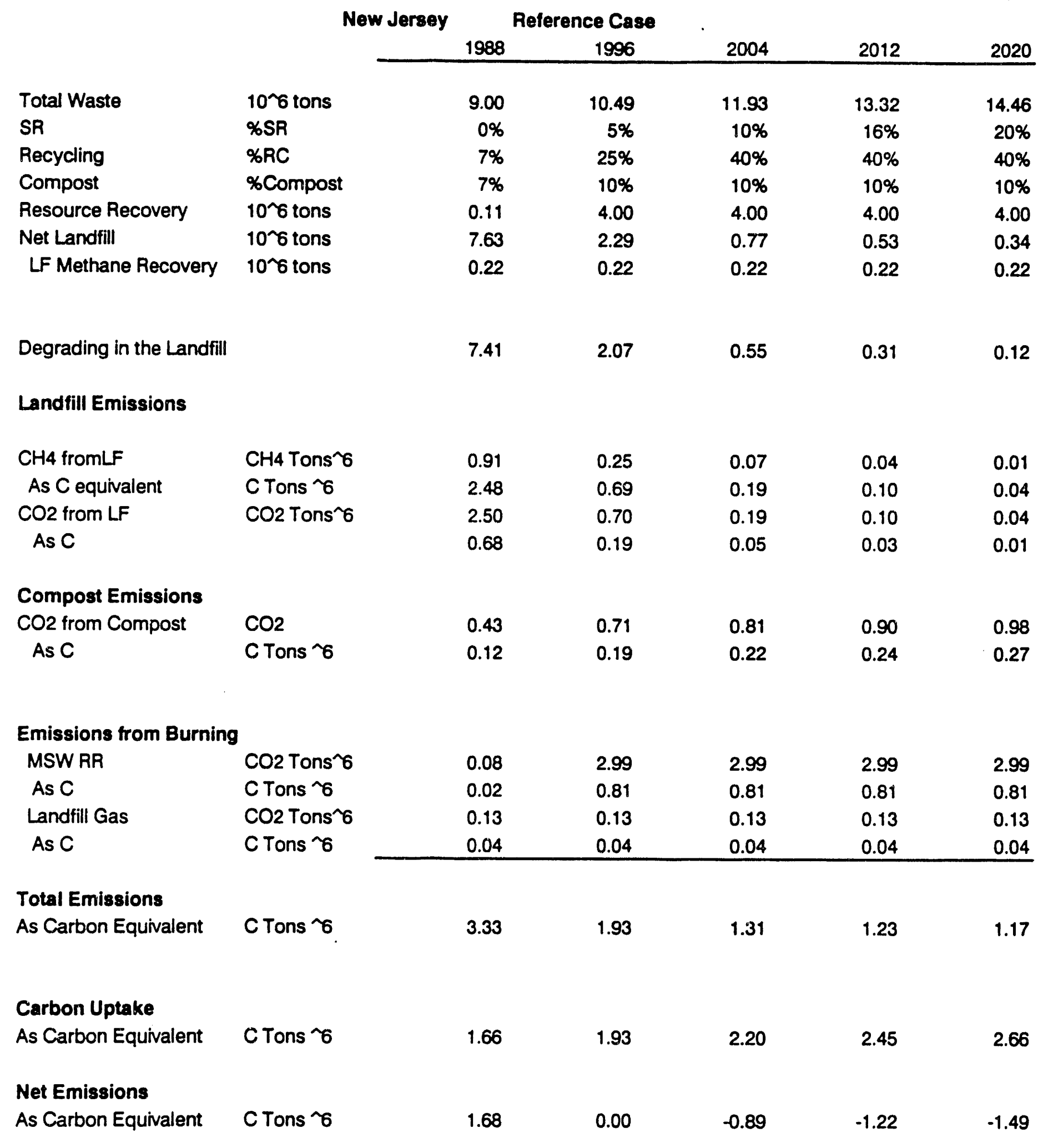




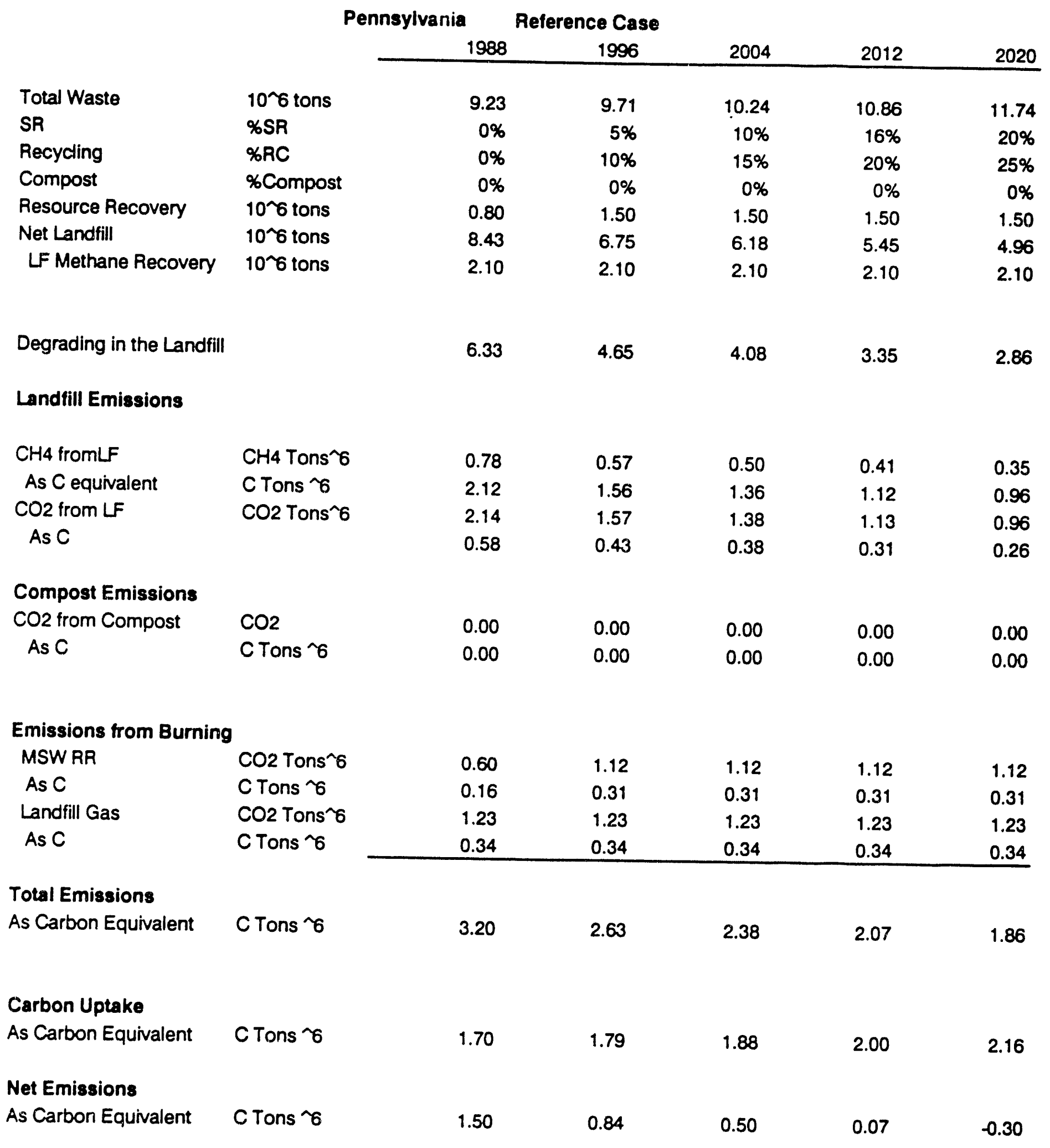




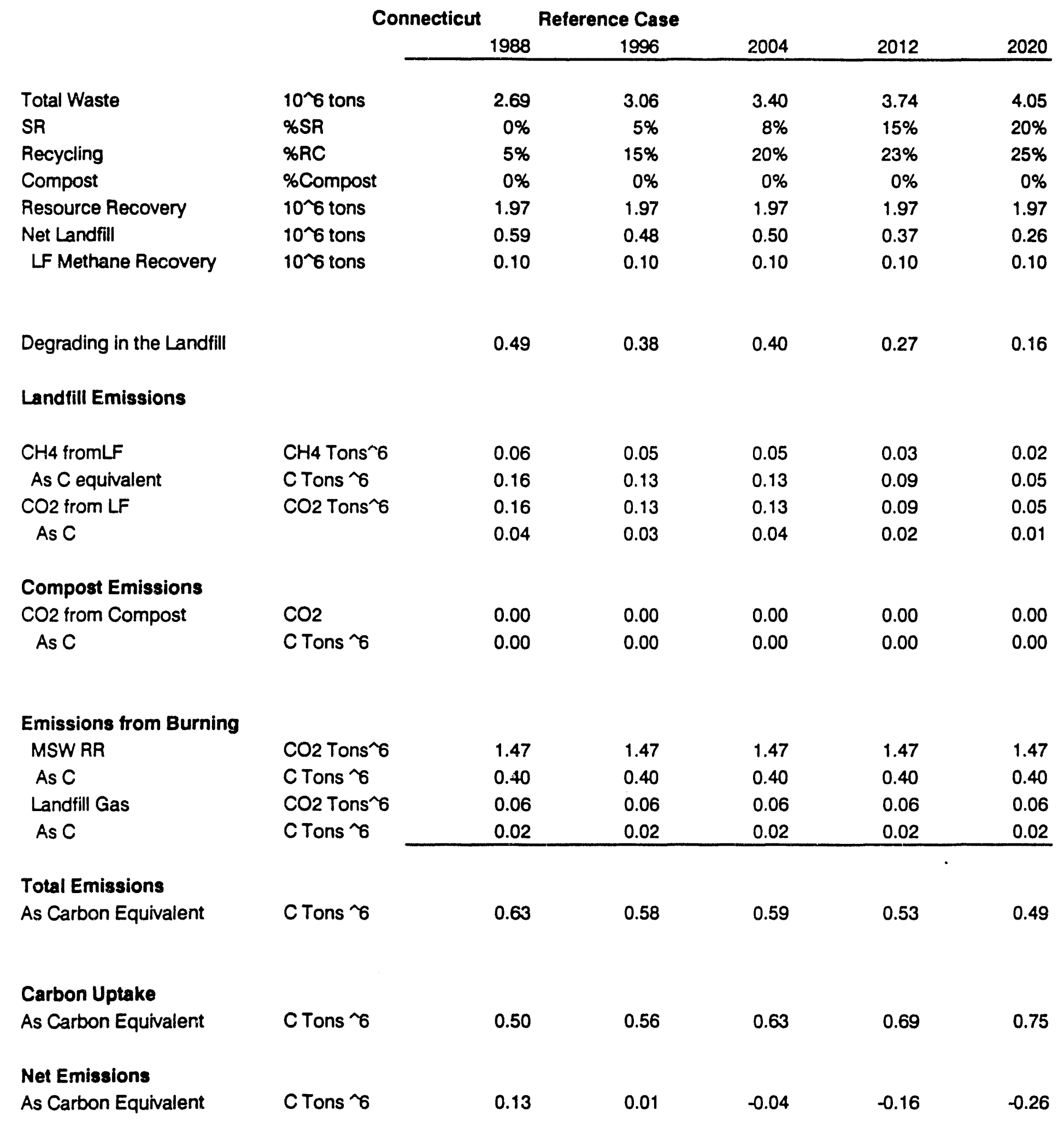


Total Waste

SR

Recycling

Compost

Resource Recovery

Net Landfill

LF Methane Recovery

Degrading in the Landfill

\section{Landfill Emissions}

$\mathrm{CH} 4$ fromLF

As $C$ equivalent

$\mathrm{CO} 2$ from LF

As C

\section{Compost Emissions}

$\mathrm{CO} 2$ from Compost

As C $10^{\wedge} 6$ tons

\%SR

\%RC

\%Compost

$10^{\wedge} 6$ tons

$10^{\circ} 6$ tons

$10^{\wedge} 6$ tons
1.01

$0 \%$

$10 \%$

$7 \%$

0.63

0.21

0.00

0.21

$\mathrm{CH}_{4}$ Tons $^{\sim} 6$

$C$ Tons $\gamma$

CO2 Tons` 6
1.14

$0 \%$

$30 \%$

$10 \%$

0.63

0.05

0.00

0.05

0.00

0.07

0.13

1.51

$0 \%$

$35 \%$

$15 \%$

0.63

0.13

0.00

0.00

0.00
0.00

0.00

0.00

0.00

0.00

0.02

0.02

0.02

0.08

0.02

0.13

0.03

0.15

0.01
0.14

0.04

0.01

0.02

0.02

0.01
0.04

0.04

0.01

0.04

\section{Emissions from Burning}

MSW RR

As $\mathrm{C}$

Landfill Gas

As $\mathrm{C}$
0.47

0.13

0.00

0.00

0.23

0.17

0.47

0.13

0.00

0.00

C Tons $\widetilde{6}$

Total Emissions

As Carbon Equivalent

\section{Carbon Uptake}

As Carbon Equivalent

Net Emissions

As Carbon Equivalent
CO2 Tons^ 6

$C$ Tons ${ }^{\wedge} 6$

$\mathrm{CO} 2$ Tons $^{\wedge} 6$

$C$ Tons ${ }^{\wedge} 6$
C Tons ${ }^{\wedge} 6$

0.04

$-0.04$

$-0.07$

$-0.06$

0.47

0.47

0.13

0.13

0.13

0.00

0.00

0.00

0.00

0.00 
Massachusetts Reference Case

Total Waste

SR

Recycling

Compost

Resource Recovery

Net Landfill

LF Methane Recovery

Degrading in the Landfill

$10^{\wedge} 6$ tons

\%SR

\%RC

\%Compost

$10^{\wedge} 6$ tons

$10^{\wedge} 6$ tons

$10^{\wedge} 6$ tons

3.19

1.94

0.33

0.70

1.04

\section{Landfill Emissions}

$\mathrm{CH} 4$ fromLF

As $C$ equivalent

$\mathrm{CO} 2$ from $L F$

As $\mathrm{C}$

\section{Compost Emissions}

$\mathrm{CO} 2$ from Compost

As C

$\mathrm{CO} 2$

C Tons ${ }^{\wedge} 6$

0.00

0.00

0.39

1.07

1.08

0.29

0.24

0.65

0.65

0.18

0.52

0.14

1.09

1.20

1.31

0.30

0.33

0.36

$10 \%$

$25 \%$

$20 \%$

3.07

1.28

0.24

0.13

0.35

0.35

0.10

\section{Emissions from Burning}

MSW RR

As $C$

Landfill Gas

As C
CO2 Tons $₹$

C Tons $₹$

CO2 Tons ${ }^{\wedge} 6$

$c$ Tons ${ }^{\wedge} 6$
2.02

0.55

0.14

0.04

1.95

1.63

1.10

1.29

1.46

Total Emissions

As Carbon Equivalen

\section{Carbon Uptake}

As Carbon Equivalent

C Tons $\mathcal{B}$

1.22

1.34

1.49

1.64

1.78

Net Emissions

As Carbon Equivalent

C Tons $` 6$

0.74

0.29

$-0.39$

$-0.35$

$-0.32$

2.29

0.63

0.14

0.04

\begin{tabular}{lll}
0.63 & 0.63 & 0.63 \\
0.04 & 0.14 & 0.14 \\
0.04 & 0.04 & 0.04 \\
\hline
\end{tabular}

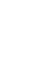


New Hampshire Reference Case

1988

1996

Total Waste

SR

Recycling

Compost

Resource Recovery

Net Landfill

LF Methane Recovery

Degrading in the Landfill

$10^{\wedge} 6$ tons

\%SR

\%RC

\%Compost

$10^{\Upsilon} 6$ tons

$10^{\wedge} 6$ tons

$10^{\wedge} 6$ tons

$\mathrm{CH} 4$ Tons $^{\wedge} 6$

C Tons 16

CO2 Tons $₹ 6$

\section{Landfill Emissions}

As $C$ equivalent

$\mathrm{CO} 2$ from $L F$
0.99

$0 \%$

$5 \%$

$0 \%$

0.31

0.63

0.08

0.55

0.15

0.12

0.10

0.19

$\mathrm{CH} 4$ fromLF

As C

\section{Compost Emissions}

CO2 from Compost

As C

$\mathrm{CO} 2$

C Tons ${ }^{\wedge} 6$

0.00

0.00

0.04

0.01

0.10

0.03

0.01

0.04

0.04

0.01

0.01

1.70

$10 \%$

$15 \%$

$15 \%$

0.84

0.18

0.08

1.85

$10 \%$

$15 \%$

$15 \%$

0.84

0.27

0.08

0.19

0.05

0.01

0.01

0.02

0.06

0.06

0.02

Emissions from Burning

$0.05 \quad 0.05$

MSW RR

As $C$

Landfill Gas

As $\mathrm{C}$

CO2 Tons ${ }^{\wedge} 6$

C Tons ${ }^{\wedge} 6$

CO2 Tons $\wedge 6$

$C$ Tons ${ }^{\top} 6$

Total Emissions

As Carbon Equivalent

$$
\text { C Tons } ₹
$$

0.31

0.26

0.26

0.27

0.31

\section{Carbon Uptake}

As Carbon Equivalen

$$
\text { C Tons ^ } 6
$$

0.18

0.2

0.27

0.31

0.34

Net Emissions

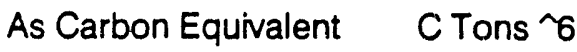

0.13

0.03

$-0.01$

$-0.04$

$-0.03$ 
Total Waste

SR

Recycling

Compost

Resource Recovery

Net Landfill

LF Methane Recovery $10^{\wedge} 6$ tons

\%SR

\%RC

\%Compost

$10 \Upsilon 6$ tons

$10^{\wedge} 6$ tons

$10^{\wedge} 6$ tons
1988

0.82

$0 \%$

$3 \%$

$3 \%$

0.06

0.72

0.19

0.53

0.28

0.01

0.08

0.10

1996

0.93

$0 \%$

$5 \%$

$0 \%$

0.41

0.47

0.19

0.20

0.19

0.27

0.19

0.19

$0 \%$

0.76

.29

Landfill Emissions

$\mathrm{CH} 4$ fromLF

As $C$ equivalent

$\mathrm{CO} 2$ from $L F$

As C

Compost Emissions

$\mathrm{CO} 2$ from Compost

As $\mathrm{C}$

$\mathrm{CO} 2$

C Tons ${ }^{\wedge} 6$
$\mathrm{CH}_{4}$ Tons $^{`} 6$

C Tons 8

CO2 Tons $₹$
0.07

0.18

0.18

0.05

0.01

0.00

\section{Emissions from Burning}

\section{MSW RR}

As $\mathrm{C}$

Landfill Gas

As C
CO2 Tons` 6

$C$ Tons ${ }^{\wedge} 6$

CO2 Tons ${ }^{\wedge} 6$

$C$ Tons ${ }^{\wedge} 6$
0.04

0.01

0.11

0.03

0.27

0.23

0.19

0.57

0.15

0.11

0.03

0.03

0.00
0.00

0.00

0.00

0.01

0.03

0.03

0.01

0.01

0.04

0.04

0.01

Total Emissions

As Carbon Equivalent

C Tons $` 6$

0.22

0.23

\section{Carbon Uptake}

As Carbon Equivalent

C Tons $\mathfrak{6}$

0.15

0.17

0.19

0.21

0.23

Net Emissions

As Carbon Equivalent $\quad C$ Tons ${ }^{\wedge} G$

0.12

0.06

0.00

0.01

0.00 


\begin{tabular}{|c|c|c|c|c|c|c|}
\hline & \multicolumn{2}{|c|}{ Vermont } & \multicolumn{4}{|c|}{ Reference Case } \\
\hline & & 1988 & 1996 & 2004 & 2012 & 2020 \\
\hline Total Waste & $10^{\wedge} 6$ tons & 0.33 & 0.37 & 0.41 & 0.45 & 0.49 \\
\hline SR & \%SR & $0 \%$ & $3 \%$ & $5 \%$ & $5 \%$ & $5 \%$ \\
\hline Recycling & \%RC & $8 \%$ & $8 \%$ & $15 \%$ & $15 \%$ & $15 \%$ \\
\hline Compost & \%Compost & $8 \%$ & $8 \%$ & $15 \%$ & $15 \%$ & $15 \%$ \\
\hline Resource Recovery & $10^{\wedge} 6$ tons & 0.06 & 0.19 & 0.19 & 0.19 & 0.19 \\
\hline Net Landfill & $10^{\wedge} 6$ tons & 0.22 & 0.12 & 0.08 & 0.11 & 0.13 \\
\hline LF Methane Recovery & $10^{\wedge} 6$ tons & 0.00 & 0.00 & 0.00 & 0.00 & 0.00 \\
\hline Degrading in the Landfill & & 0.22 & 0.12 & 0.08 & 0.11 & 0.13 \\
\hline \multicolumn{7}{|l|}{ Landfill Emissions } \\
\hline $\mathrm{CH} 4$ fromLF & CH4 Tons^ 6 & 0.03 & 0.01 & 0.01 & 0.01 & 0.02 \\
\hline As $C$ equivalent & C Tons ${ }^{\wedge} 6$ & 0.07 & 0.04 & 0.03 & 0.04 & 0.04 \\
\hline $\mathrm{CO} 2$ from LF & CO2 Tons^6 & 0.07 & 0.04 & 0.03 & 0.04 & 0.05 \\
\hline As C & & 0.02 & 0.01 & 0.01 & 0.01 & 0.01 \\
\hline \multicolumn{7}{|l|}{ Compost Emissions } \\
\hline CO2 from Compost & $\mathrm{CO} 2$ & 0.02 & 0.02 & 0.04 & 0.05 & 0.05 \\
\hline As C & $C$ Tons $₹ 6$ & 0.00 & 0.01 & 0.01 & 0.01 & 0.01 \\
\hline \multicolumn{7}{|c|}{ Emissions from Burning } \\
\hline MSW RR & CO2 Tons` 6 & 0.04 & 0.14 & 0.14 & 0.14 & 0.14 \\
\hline As $\mathrm{C}$ & C Tons ${ }^{\wedge} 6$ & 0.01 & 0.04 & 0.04 & 0.04 & 0.04 \\
\hline Landfill Gas & $\mathrm{CO} 2$ Tons $^{`} 6$ & 0.00 & 0.00 & 0.00 & 0.00 & 0.00 \\
\hline As $\mathrm{C}$ & C Tons ^ 6 & 0.00 & 0.00 & 0.00 & 0.00 & 0.00 \\
\hline \multicolumn{7}{|l|}{ Total Emissions } \\
\hline As Carbon Equivalent & CTons $\mathfrak{B}$ & 0.11 & 0.09 & 0.08 & 0.10 & 0.11 \\
\hline \multicolumn{7}{|l|}{ Carbon Uptake } \\
\hline As Carbon Equivalent & $C$ Tons ^ 6 & 0.06 & 0.07 & 0.08 & 0.08 & 0.09 \\
\hline \multicolumn{7}{|l|}{ Net Emissions } \\
\hline As Carbon Equivalent & C Tons ^ 6 & 0.05 & 0.03 & 0.01 & 0.01 & 0.02 \\
\hline
\end{tabular}




\section{New England}

\section{CONEG Region Solid Waste System}

\section{Greenhouse Gas Emissions}

(Million Tons)

Policy Case

$\begin{array}{lllll}1988 & 1996 & 2004 & 2012 & 2020\end{array}$

Landfill Emissions

\begin{tabular}{llllll}
$\mathrm{CO} 2$ & 1.83 & 1.01 & 2.12 & 2.34 & 2.54 \\
$\mathrm{CH} 4$ & 0.75 & 0.20 & 0.00 & 0.00 & 0.00 \\
\cline { 2 - 6 } C Equiv & 2.14 & 0.78 & 0.56 & 0.62 & 0.67
\end{tabular}

Res Recovery Emissions

$\mathrm{CO} 2$
$\mathrm{C}$ Equin

Total C Equivalent

\begin{tabular}{lllll}
4.65 & 5.53 & 4.01 & 3.14 & 2.71 \\
\hline 1.27 & 1.51 & 1.09 & 0.86 & 0.74 \\
& & & & \\
3.41 & 2.29 & 1.66 & 1.48 & 1.41
\end{tabular}

Mid Atlantic

Landfill Emissions

\begin{tabular}{llllll}
$\mathrm{CO} 2$ & 6.53 & 3.37 & 4.21 & 4.47 & 4.77 \\
$\mathrm{CH} 4$ & 2.20 & 0.90 & 0.10 & 0.05 & 0.03 \\
\cline { 2 - 6 } $\mathrm{C}$ Equiv & 7.79 & 3.39 & 1.42 & 1.36 & 1.37
\end{tabular}

Res Recovery Emissions

$\mathrm{CO} 2$
$\mathrm{C}$ Equiv

Total C Equivalent

\begin{tabular}{lllll}
3.44 & 6.80 & 6.59 & 5.26 & 4.63 \\
\hline 0.94 & 1.85 & 1.80 & 1.43 & 1.26
\end{tabular}

$\begin{array}{lllll}8.73 & 5.24 & 3.22 & 2.80 & 2.63\end{array}$

New York

Landfill Emissions

\begin{tabular}{llllll}
$\mathrm{CO} 2$ & 4.71 & 3.98 & 4.48 & 4.14 & 4.14 \\
$\mathrm{CH} 4$ & 1.71 & 1.18 & 0.46 & 0.23 & 0.12 \\
\cline { 2 - 6 } $\mathrm{C}$ Equiv & 5.96 & 4.29 & 2.46 & 1.75 & 1.46
\end{tabular}

Res Recovery Emissions

$\mathrm{CO} 2$
$\mathrm{C}$ Equiv

Total C Equivalent

\begin{tabular}{lllll}
2.95 & 3.40 & 3.40 & 3.40 & 3.40 \\
\hline 0.80 & 0.93 & 0.93 & 0.93 & 0.93
\end{tabular}

$\begin{array}{lllll}6.76 & 5.22 & 3.39 & 2.67 & 2.38\end{array}$

CONEG Region

Landfill Emissions

\begin{tabular}{lrrrrr}
$\mathrm{CO} 2$ & 13.07 & 8.36 & 10.81 & 10.96 & 11.45 \\
$\mathrm{CH} 4$ & 4.67 & 2.28 & 0.56 & 0.28 & 0.15 \\
\hline C Equiv & 15.89 & 8.46 & 4.45 & 3.73 & 3.50
\end{tabular}

Res Recovery Emissions
$\mathrm{CO} 2$
C Equin

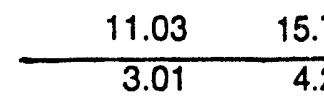

$18.90 \quad 12.75$

\begin{tabular}{rrrr}
15.73 & 14.00 & 11.80 & 10.74 \\
\hline 4.29 & 3.82 & 3.22 & 2.93
\end{tabular}

Total C Equivalent 


\begin{tabular}{|c|c|c|c|c|c|c|}
\hline & \multicolumn{2}{|c|}{ NEW YORK } & Policy Case & \multirow[b]{2}{*}{2004} & \multirow[b]{2}{*}{2012} & \multirow[b]{2}{*}{2020} \\
\hline & & 1988 & 1996 & & & \\
\hline Total Waste & $10^{\Upsilon} 6$ tons & 20.26 & 21.94 & 23.94 & 26.07 & 28.24 \\
\hline SR & $\% S R$ & $0 \%$ & $10 \%$ & $10 \%$ & $15 \%$ & $20 \%$ \\
\hline Recycling & $\% R C$ & $11 \%$ & $20 \%$ & $35 \%$ & $40 \%$ & $40 \%$ \\
\hline Compost & \%Compost & $0 \%$ & $5 \%$ & $20 \%$ & $20 \%$ & $20 \%$ \\
\hline Resource Recovery & $10^{\wedge} 6$ tons & 3.50 & 4.10 & 4.10 & 4.10 & 4.10 \\
\hline Net Landfill & $10^{\sim} 6$ tons & 14.54 & 10.16 & 4.28 & 2.42 & 1.55 \\
\hline LF Methane Recovery & $10 \Upsilon 6$ tons & 0.57 & 0.57 & 0.57 & 0.57 & 0.57 \\
\hline Degrading in the Landfill & & 13.97 & 9.59 & 3.71 & 1.85 & 0.98 \\
\hline \multicolumn{7}{|l|}{ Landfill Emissions } \\
\hline $\mathrm{CH} 4$ fromLF & $\mathrm{CH}_{4}$ Tons $^{`} 6$ & 1.71 & 1.18 & 0.46 & 0.23 & 0.12 \\
\hline As $C$ equivalent & $C$ Tons ${ }^{\top}$ & 4.67 & 3.21 & 1.24 & 0.62 & 0.33 \\
\hline CO2 from LF & CO2.Tons` 6 & 4.71 & 3.24 & 1.25 & 0.62 & 0.33 \\
\hline As C & & 1.28 & 0.88 & 0.34 & 0.17 & 0.09 \\
\hline \multicolumn{7}{|l|}{ Compost Emissions } \\
\hline CO2 from Compost & $\mathrm{CO} 2$ & 0.00 & 0.74 & 3.23 & 3.52 & 3.81 \\
\hline As C & C Tons ^ 6 & 0.00 & 0.20 & 0.88 & 0.96 & 1.04 \\
\hline \multicolumn{7}{|l|}{ Emissions from Burning } \\
\hline MSW RR & CO2 Tons^ 6 & 2.61 & 3.06 & 3.06 & 3.06 & 3.06 \\
\hline As $C$ & C Tons $` 6$ & 0.71 & 0.84 & 0.84 & 0.84 & 0.84 \\
\hline Landfill Gas & CO2 Tons^ 6 & 0.33 & 0.33 & 0.33 & 0.33 & 0.33 \\
\hline As C & C Tons ${ }^{\wedge} 6$ & 0.09 & 0.09 & 0.09 & 0.09 & 0.09 \\
\hline \multicolumn{7}{|l|}{ Total Emissions } \\
\hline As Carbon Equivalent & C Tons ^ 6 & 6.76 & 5.22 & 3.39 & 2.67 & 2.38 \\
\hline \multicolumn{7}{|l|}{ Carbon Uptake } \\
\hline As Carbon Equivalent & C Tons ^ 6 & 3.73 & 4.04 & 4.41 & 4.80 & 5.20 \\
\hline \multicolumn{7}{|l|}{ Net Emissions } \\
\hline As Carbon Equivalent & C Tons ^ 6 & 3.03 & 1.18 & -1.02 & -2.12 & -2.81 \\
\hline
\end{tabular}




\begin{tabular}{rrrrrr} 
Maryland & \multicolumn{3}{c}{ Policy Case } & & \\
& 1988 & 1996 & 2004 & 2012 & 2020 \\
\hline 5.47 & 5.10 & 5.93 & 6.73 & 7.32 \\
$0 \%$ & $10 \%$ & $10 \%$ & $15 \%$ & $20 \%$ \\
$0 \%$ & $20 \%$ & $35 \%$ & $40 \%$ & $40 \%$ \\
$0 \%$ & $5 \%$ & $20 \%$ & $20 \%$ & $20 \%$ \\
1.09 & 1.09 & 1.09 & 1.09 & 1.09 \\
4.38 & 2.23 & 0.99 & 0.59 & 0.37 \\
0.16 & 0.16 & 0.16 & 0.16 & 0.16
\end{tabular}

Degrading in the Landfill

4.22

2.07

0.83

0.43

0.21

Landfill Emissions

$\mathrm{CH} 4$ fromLF

As $C$ equivalent

$\mathrm{CO} 2$ from LF

As C

\section{Compost Emissions}

$\mathrm{CO} 2$ from Compost

As C
$\mathrm{CH}_{4}$ Tons $^{`} 6$

$C$ Tons 16

$\mathrm{CO} 2$ Tons` 6
0.52

1.41

1.42

0.39

$\mathrm{CO} 2$

C Tons $\uparrow$
0.00

0.00
0.25

0.69

0.70

0.19

0.17

0.05
0.10

0.28

0.28

0.08

0.80

0.22

0.91

0.05

0.03

0.14

0.07

0.15

0.07

0.04

0.02

\section{Emissions from Burning}

MSW RR

As $\mathrm{C}$

Landfill Gas

As $\mathrm{C}$
CO2 Tons^ 6

C Tons ${ }^{\circ} 6$

$\mathrm{CO} 2$ Tons $^{\wedge} 6$

$c$ Tons ${ }^{\wedge} 6$
0.81

0.22

0.09

0.03

C Tons ${ }^{\wedge} 6$
2.05

1.18

0.82

0.68

0.61

\section{Carbon Uptake}

As Carbon Equivalent $\quad C$ Tons ${ }^{\wedge} 6$

1.01

0.94

1.09

1.24

1.35

Net Emissions

As Carbon Equivalent $\quad C$ Tons ${ }^{\circ} 6$

1.04

0.24

$-0.27$

$-0.56$

$-0.74$

Total Emissions 


Total Waste
SR
Recycling
Compost
Resource Recovery
Net Landfill
LF Methane Recovery

Degrading in the Landfill

\section{Landfill Emissions}

$\mathrm{CH} 4$ fromLF
As $\mathrm{C}$ equivalent
$\mathrm{CO} 2$ from $\mathrm{LF}$
$\mathrm{As} \mathrm{C}$

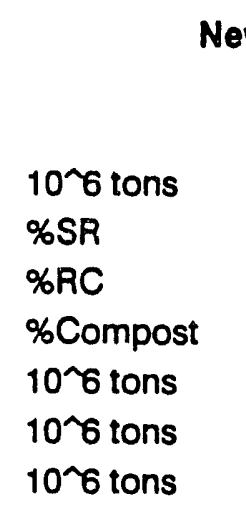

New Jersey

Policy Case

\begin{tabular}{rrrrr}
1988 & 1996 & 2004 & 2012 & 2020 \\
\hline 9.00 & 10.49 & 11.93 & 13.32 & 14.46 \\
$0 \%$ & $10 \%$ & $10 \%$ & $15 \%$ & $20 \%$ \\
$7 \%$ & $20 \%$ & $35 \%$ & $40 \%$ & $40 \%$ \\
$7 \%$ & $5 \%$ & $20 \%$ & $20 \%$ & $20 \%$ \\
0.11 & 4.00 & 3.96 & 3.11 & 2.67 \\
7.63 & 2.82 & 0.22 & 0.22 & 0.22 \\
0.22 & 0.22 & 0.22 & 0.22 & 0.22
\end{tabular}

\section{Compost Emissions}

$\mathrm{CO} 2$ from Compost

As C

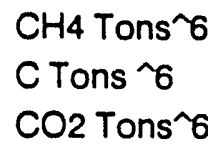

7.41

2.60

0.00

0.00

0.00

0.91

2.48

2.50

0.68

0.32

0.87

0.88

0.24

0.00

0.00

0.00

0.00

0.00

0.00

0.00

0.00

0.00

0.00

0.00

0.00

\section{Emissions from Burning}

MSW RR

As C

Landfill Gas

As C
CO2 Tons ${ }^{\wedge} 6$

$C$ Tons ${ }^{\wedge} 6$

CO2 Tons^ 6

$C$ Tons ${ }^{\top} 6$

$C$ Tons ${ }^{\wedge} 6$

Total Emissions

As Carbon Equivalent
$\mathrm{CO} 2$

$C$ Tons ${ }^{\wedge} 6$

0.43

3.33

0.08

0.02

0.13

0.04

0.35

0.10

1.61

0.44

1.80

0.49

1.95

0.53

\section{Carbon Uptake}

As Carbon Equivalent

$C$ Tons ${ }^{\wedge} 6$

1.66

1.93

2.20

2.45

2.66

Net Emissions

As Carbon Equivalen

C Tons ${ }^{\wedge} 6$

1.68

0.12

$-0.92$

$-1.29$

$-1.55$ 
Total Waste

$10^{\wedge} 6$ tons

\%SR

SR

Recycling

Compost

Resource Recovery

Net Landfill

LF Methane Recovery

\%RC

\%Compost

$10^{\Upsilon} 6$ tons

$10^{\wedge} 6$ tons

$10^{\wedge} 6$ tons
9.23

9.71

0.00

0.00

\section{Landfill Emissions}

$\mathrm{CH} 4$ fromis $\mathrm{F}$

As $C$ equivalent

$\mathrm{CO} 2$ from $L F$

As $\mathrm{C}$
$0 \%$

$0 \%$

$0 \%$

0.80

8.43

2.10

$10 \%$

$20 \%$

$5 \%$

1.50

4.81

2.10

6.33

2.71

0.00

$\mathrm{CH}_{4}$ Tons $^{\top} 6$

$C$ Tons 16

$\mathrm{CO} 2$ Tons $^{\wedge} G$
0.78

2.12

2.14

0.58

0.00

0.00
0.33

0.91

0.91

0.25

0.33

0.09
10.24

$10 \%$

$35 \%$

$20 \%$

1.48

2.10

2.10

$\mathrm{CO} 2$

$c$ Tons $₹$

As $\mathrm{C}$

Compost Emissions

$\mathrm{CO} 2$ from Compost

$$
\text { As }
$$

\section{Emissions from Burning}

\section{MSW RR}

As $\mathrm{C}$

Landfill Gas

As C
CO2 Tons^ 6

C Tons 16

CO2 Tons^ 6

$C$ Tons ${ }^{\top} 6$
0.60

0.16

1.23

0.34

3.20

As Carbon Equivalent

C Tons $₹$

1.89

1.02

0.86

0.82

\section{Carbon Uptake}

As Carbon Equivalent

C Tons 96

1.70

1.79

1.88

2.00

2.16

Net Emissions

As Carbon Equivalent $\quad$ C Tons $₹$

1.50

0.10

$-0.87$

$-1.14$

$-1.34$ 


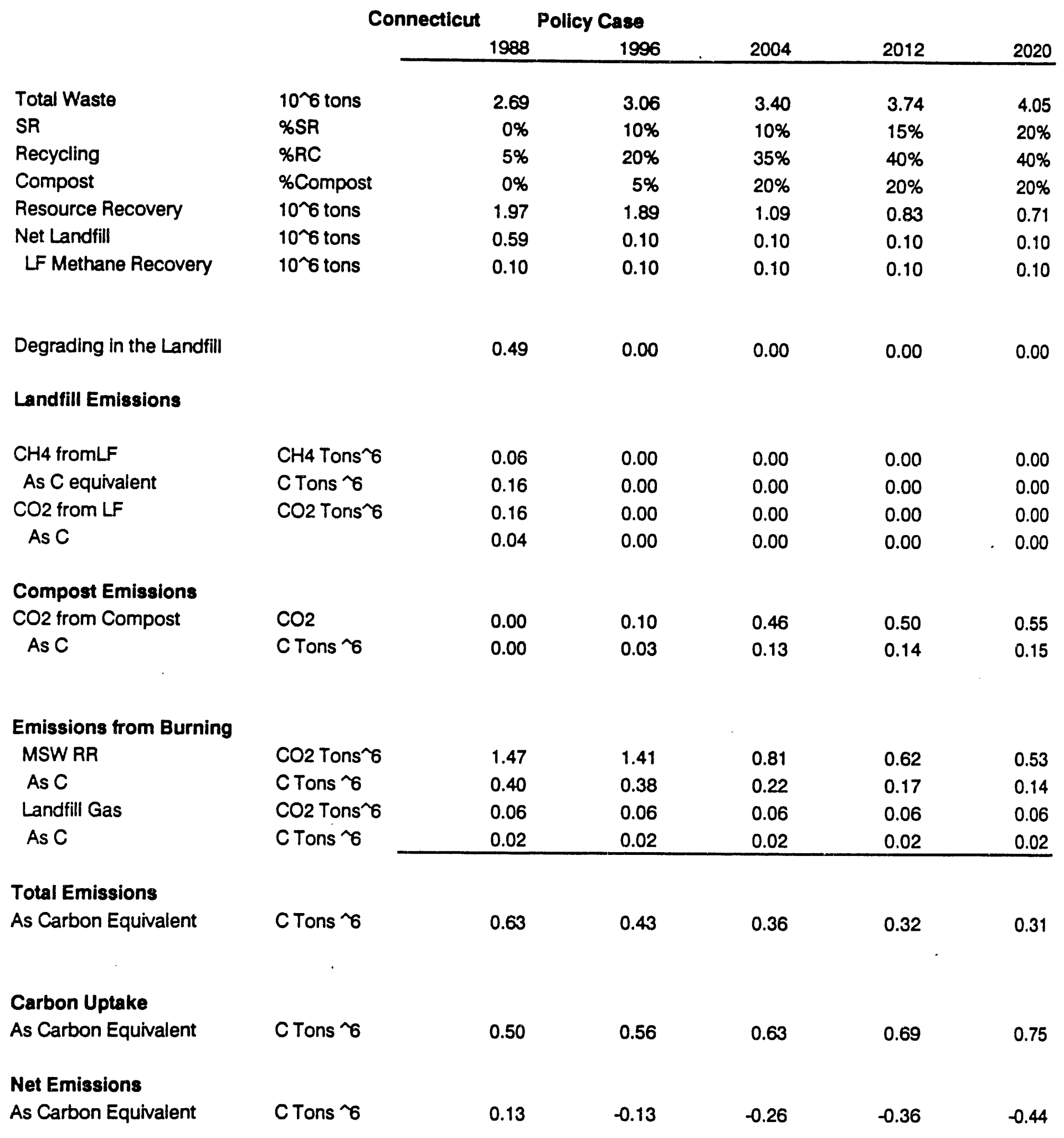


Total Waste

SR

Recycling

Compost

Resource Recovery

Net Landfill

LF Methane Recovery

Degrading in the Landfill

$\mathrm{CH} 4$ fromLF

As $C$ equivalent

$\mathrm{CO} 2$ from $L F$

As C

\section{Compost Emissions}

$\mathrm{CO} 2$ from Compost

As C

$\mathrm{CO} 2$

$c$ Tons ${ }^{\wedge} 6$

0.01

\section{Landfill Emissions}

1.14 $10 \%$ $20 \%$ $5 \%$ 0.63

0.11

0.00

0.11

$\mathrm{CH}_{4}$ Tons $^{\wedge} 6$

$C$ Tons ${ }^{\wedge} 6$

CO2 Tons ${ }^{\wedge} 6$
0.05

0.03

0.07

0.07

0.02
0.01

0.04

0.04

0.01

0.04

0.01
1.26

$10 \%$

$35 \%$

$20 \%$

0.44

0.00

0.00

0.00

0.00

0.00

0.00

0.00

0.17

0.05
1.39

$15 \%$

$40 \%$

$20 \%$

0.35

0.00

0.00

0.00

0.00

1.51

$20 \%$

$40 \%$

$20 \%$

0.30

0.00

0.00

\section{Emissions from Burning}

MSW RR

As $C$

Landfill Gas

As $\mathrm{C}$

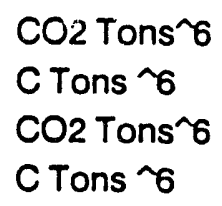

0.47

0.13

0.00

0.00

0.47

0.13

0.00

0.00

0.00

0.00

0.00

0.00

0.00

0.00

0.00

0.00

\section{Total Emissions}

As Carbon Equivalent

C Tons ${ }^{\wedge} 6$

0.23

0.19

0.14

0.12

0.12

\section{Carbon Uptake}

As Carbon Equivalent

$$
\text { C Tons }{ }^{\wedge}
$$

0.19

0.21

0.23

0.26

0.28

Net Emissions

As Carbon Equivalent

C Tons ^ 6

0.04

$-0.02$

$-0.10$

$-0.13$

$-0.16$ 


\begin{tabular}{|c|c|c|c|c|c|c|}
\hline & \multicolumn{2}{|c|}{ Massachusetts } & Policy Case & \multirow[b]{2}{*}{2004} & \multirow[b]{2}{*}{2012} & \multirow[b]{2}{*}{2020} \\
\hline & & 1988 & 1996 & & & \\
\hline Total Waste & $10^{\wedge} 6$ tons & 6.61 & 7.29 & 8.10 & 8.92 & 9.67 \\
\hline SR & \%SR & $0 \%$ & $10 \%$ & $10 \%$ & $15 \%$ & $20 \%$ \\
\hline Recycling & \%RC & $7 \%$ & $20 \%$ & $35 \%$ & $40 \%$ & $40 \%$ \\
\hline Compost & \%Compost & $0 \%$ & $5 \%$ & $20 \%$ & $20 \%$ & $20 \%$ \\
\hline Resource Recovery & $10^{\wedge} 6$ tons & 2.71 & 3.07 & 2.59 & 1.99 & 1.69 \\
\hline Net Landfill & $10^{\wedge} 6$ tons & 3.43 & 1.67 & 0.24 & 0.24 & 0.24 \\
\hline LF Methane Recovery & $10^{\sim} 6$ tons & 0.24 & 0.24 & 0.24 & 0.24 & 0.24 \\
\hline Degrading in the Landfill & & 3.19 & 1.43 & 0.00 & 0.00 & 0.00 \\
\hline \multicolumn{7}{|l|}{ Landfill Emissions } \\
\hline $\mathrm{CH} 4$ fromLF & $\mathrm{CH}_{4}$ Tons` 6 & 0.39 & 0.18 & 0.00 & 0.00 & 0.00 \\
\hline As $C$ equivalent & $C$ Tons ${ }^{\wedge} 6$ & 1.07 & 0.48 & 0.00 & 0.00 & 0.00 \\
\hline CO2 from LF & CO2 Tons^ 6 & 1.08 & 0.48 & 0.00 & 0.00 & 0.00 \\
\hline As C & & 0.29 & 0.13 & 0.00 & 0.00 & 0.00 \\
\hline \multicolumn{7}{|l|}{ Compost Emissions } \\
\hline CO2 from Compost & $\mathrm{CO} 2$ & 0.00 & 0.25 & 1.09 & 1.20 & 1.31 \\
\hline As C & C Tons ^ 6 & 0.00 & 0.07 & 0.30 & 0.33 & 0.36 \\
\hline \multicolumn{7}{|c|}{ Emissions from Burning } \\
\hline MSW RR & CO2 Tons^6 & 2.02 & 2.29 & 1.94 & 1.49 & 1.27 \\
\hline As C & $C$ Tons ${ }^{\wedge} 6$ & 0.55 & 0.63 & 0.53 & 0.41 & 0.35 \\
\hline Landfill Gas & CO2 Tons^6 & 0.14 & 0.14 & 0.14 & 0.14 & 0.14 \\
\hline As C & $C$ Tons ${ }^{\top} 6$ & 0.04 & 0.04 & 0.04 & 0.04 & 0.04 \\
\hline \multicolumn{7}{|l|}{ Total Emissions } \\
\hline As Carbon Equivalent & $C$ Tons ^ 6 & 1.95 & 1.34 & 0.86 & 0.77 & 0.74 \\
\hline & & & & & & \\
\hline \multicolumn{7}{|l|}{ Carbon Uptake } \\
\hline As Carbon Equivalent & 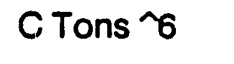 & 1.22 & 1.34 & 1.49 & 1.64 & 1.78 \\
\hline \multicolumn{7}{|l|}{ Net Emissions } \\
\hline As Carbon Equivalent & CTons ${ }^{\wedge} 6$ & 0.74 & 0.00 & -0.62 & -0.87 & -1.04 \\
\hline
\end{tabular}


Total Waste

SR

Recycling

C. npost

Resource Recovery

Net Landfill

LF Methane Recovery

Degrading in the Landfill

\section{Landfill Emissions}

$\mathrm{CH} 4$ fromLF

As $C$ equivalent

CO2 from LF

As $\mathrm{C}$

\section{Compost Emissions}

$\mathrm{CO} 2$ from Compost

As $\mathrm{C}$
$10 \Upsilon 6$ tons

\%SR

\%RC

\%Compost

$10 \wedge 6$ tons

$10^{\wedge} 6$ tons

$10^{\wedge} 6$ tons
0.99

$0 \%$

$5 \%$

$0 \%$

0.31

0.63

0.08

0.55

CH4 Tons^ 6

C Tons ${ }^{\wedge} 6$

CO2 Tons` 6

$\mathrm{CO} 2$

C Tons ${ }^{\wedge} 6$
0.00

0.00
1.26

$10 \%$

$20 \%$

$5 \%$

0.74

0.08

0.08

0.00

0.00

0.00

0.00

1.85

$20 \%$

$40 \%$

$20 \%$

0.29

0.08

0.08

0.08
0.00

0.00

0.00

0.00

0.00

0.20

0.05

0.23

0.06

0.25

0.07

0.00

0.00

0.00

0.00

\section{Emissions from Burning}

MSW RR

As $C$

Landfill Gas

As $\mathrm{C}$

Total Emissions

As Carbon Equivalent $\quad C$ Tons ${ }^{\wedge} 6$

CO2 Tons^6

C Tons ${ }^{\wedge} 6$

CO2 Tons $\wedge 6$

$c$ Tons ${ }^{\wedge} 6$

0.23

0.06

0.05

0.01

0.01

0.05

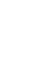

0.33

0.09

0.05

0.01

0.01

0.31

0.17

0.16

0.15

0.14

\section{Carbon Uptake}

As Carbon Equivalent $C$ Tons ` 6

0.18

0.23

0.27

0.31

0.34

Net Emissions

As Carbon Equivalent

c Tons ^ 6

0.13

$-0.06$

$-0.12$

$-0.17$

$-0.20$

0.22

0.06

0.05

0.01

0.01

0.01

0.34 


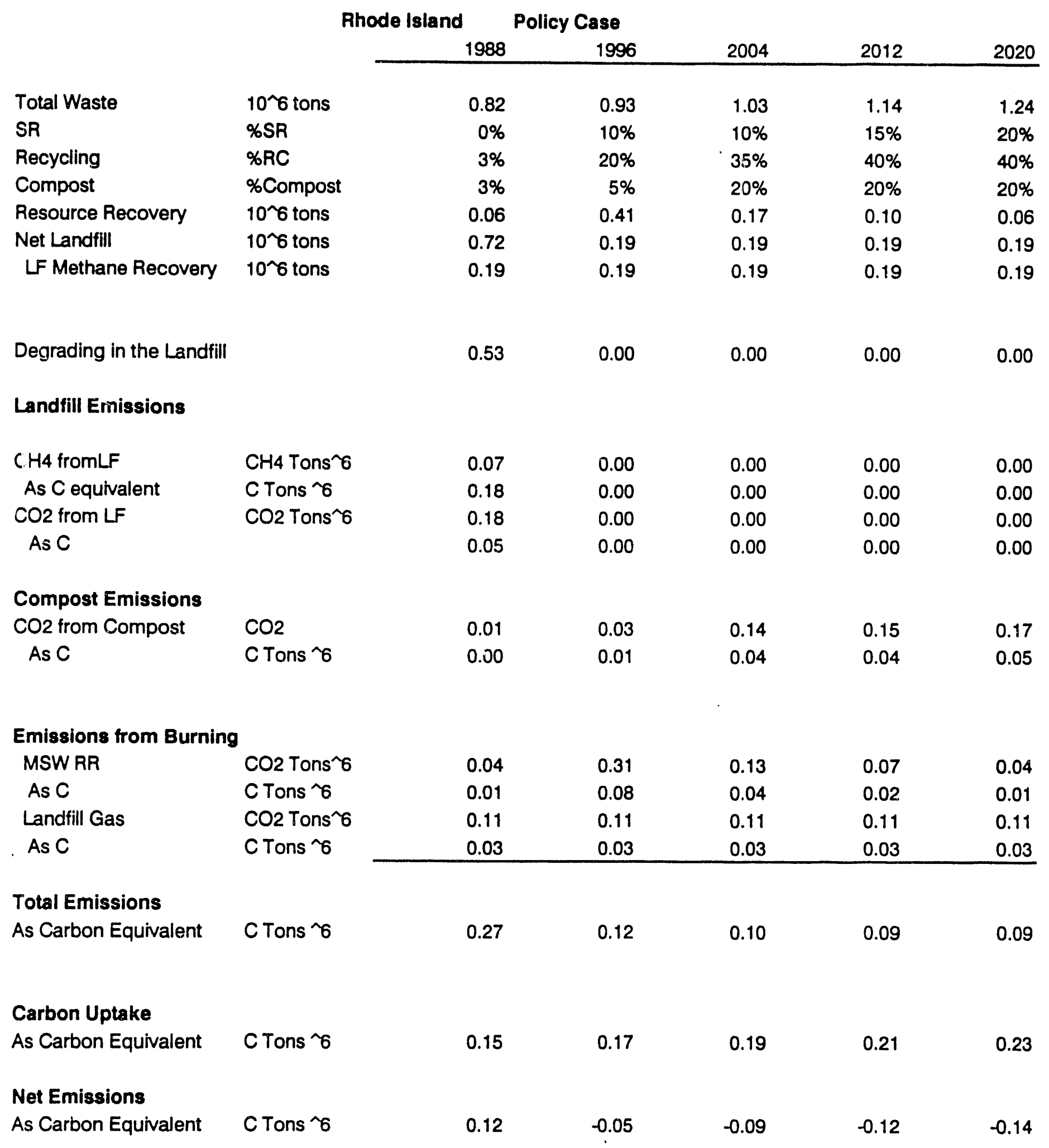


Total Waste

SR

Recycling

Compost

Resource Recovery

Net Landfill

LF Methane Recovery

Degrading in the Landfill

Landfill Emissions

$\mathrm{CH} 4$ fromLF

As $C$ equivalent

$\mathrm{CO} 2$ from $L F$

As $\mathrm{C}$

$\mathrm{CH}_{4}$ Tons` 6

$C$ Tons ${ }^{\top} 6$

CO2 Tons^ 6

0.22

0.05

0.00

0.00

0.00

0.33

$8 \%$

0.06

0.22

0.00

0.05

0.00

0.41

$10 \%$

$35 \%$

$20 \%$

0.14

0.00

0.00

0.45

$15 \%$

$40 \%$

$20 \%$

0.11

0.00

0.00

0.49

$20 \%$

$40 \%$

$20 \%$

0.10

0.00

0.00

Compost Emissions

$\mathrm{CO} 2$ from Compost

As $\mathrm{C}$

$\mathrm{CO} 2$

C Tons 16

0.02

0.00

0.01

0.00

0.06

0.02

0.06

0.02

0.07

0.02

0.00

0.00

0.00

0.00

$0.00^{\circ}$

\section{Emissions from Burning}

\section{MSW RR \\ As $\mathrm{C}$}

Landfill Gas

As $\mathrm{C}$
CO2 Tons $^{\wedge} 6$

$C$ Tons ${ }^{\top} 6$

CO2 Tons ${ }^{\wedge} 6$

$c$ Tons ${ }^{\wedge} 6$
0.04

0.01

0.00

0.00

Total Emissions

As Carbon Equivalent

C Tons ${ }^{\wedge} 6$

0.11

0.06

0.04

0.04

0.04

\section{Carbon Uptake}

As Carbon Equivalent

C Tons ^ 6

0.06

0.07

0.08

0.08

0.09

Net Emissions

As Carbon Equivalent $\quad C$ Tons ${ }^{`} \sigma$

0.08

0.07

0.02

0.00

0.00

$\begin{array}{ll}0.00 & 0.00 \\ 0.00 & 0.00\end{array}$

0.04

0.05

0.00

$-0.03$

$-0.04$ 


\section{APPENDIX B}

ASSUMPTIONS OF CONEG STATE LAND USE, FOREST BIOMASS, AND WOOD USES 
疍 邑

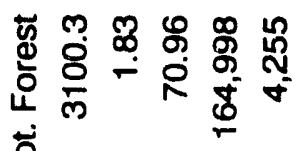

ธั่

产

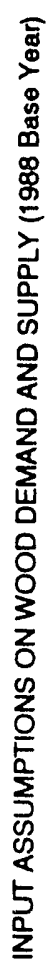

a

密点

응

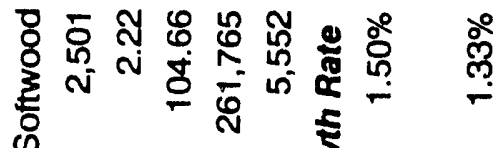

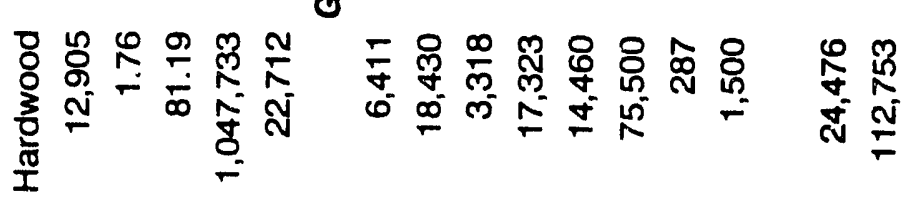

这

ठํ்

홍ㅇㅇㅇ

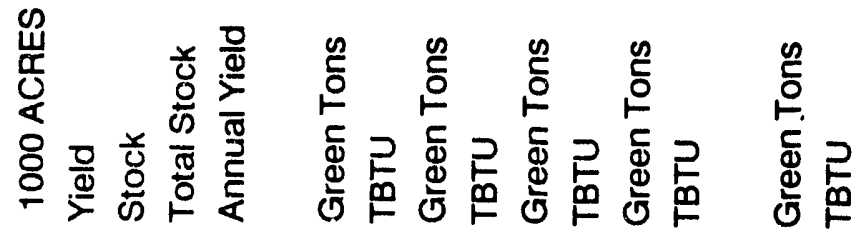

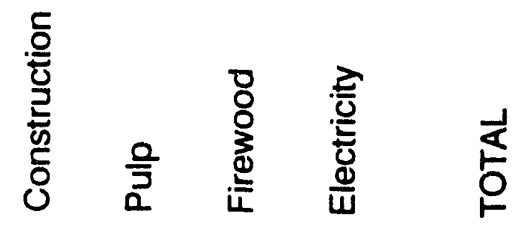

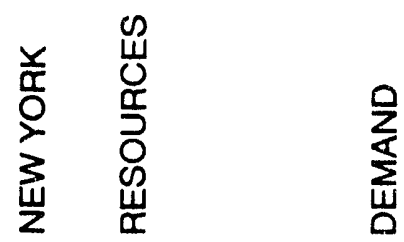




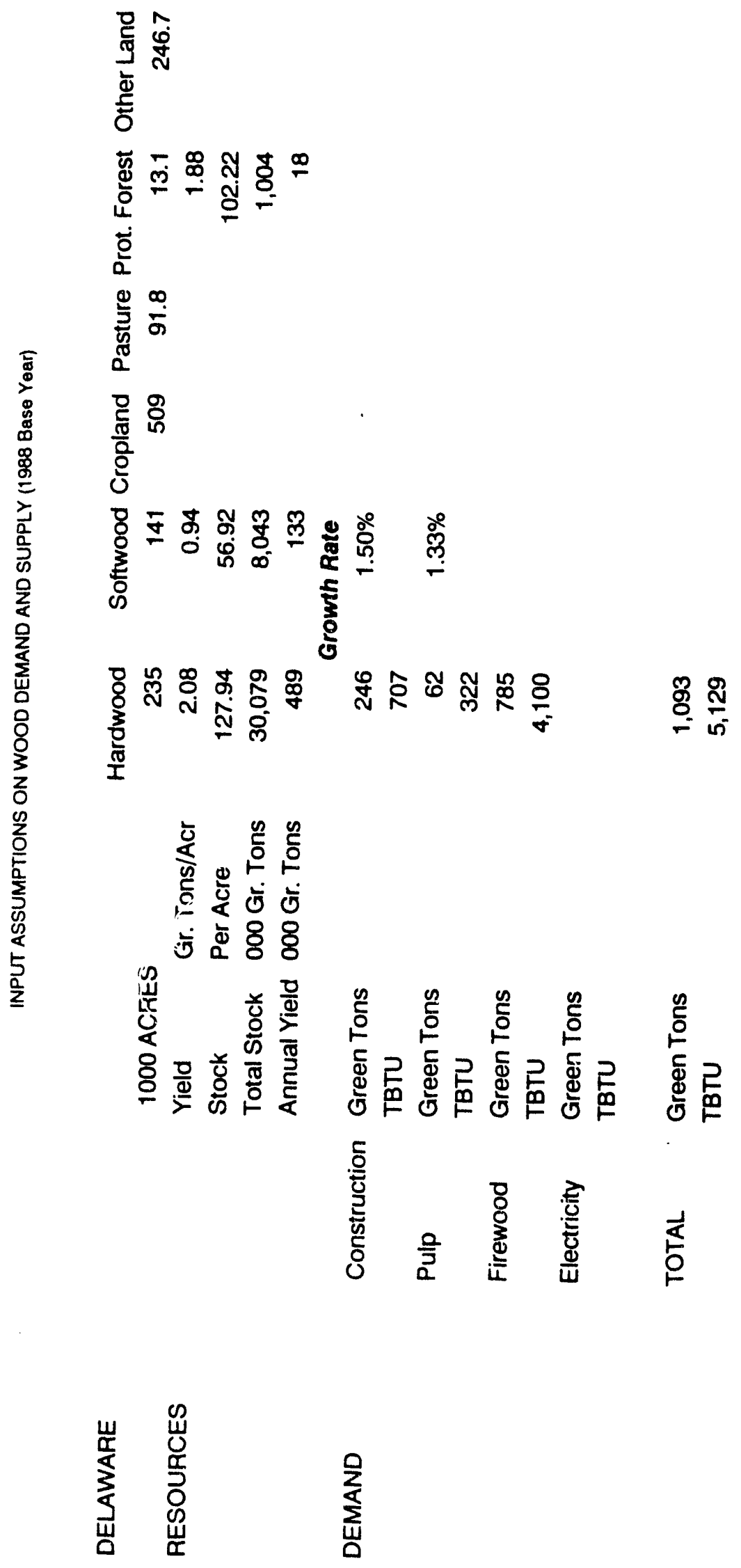




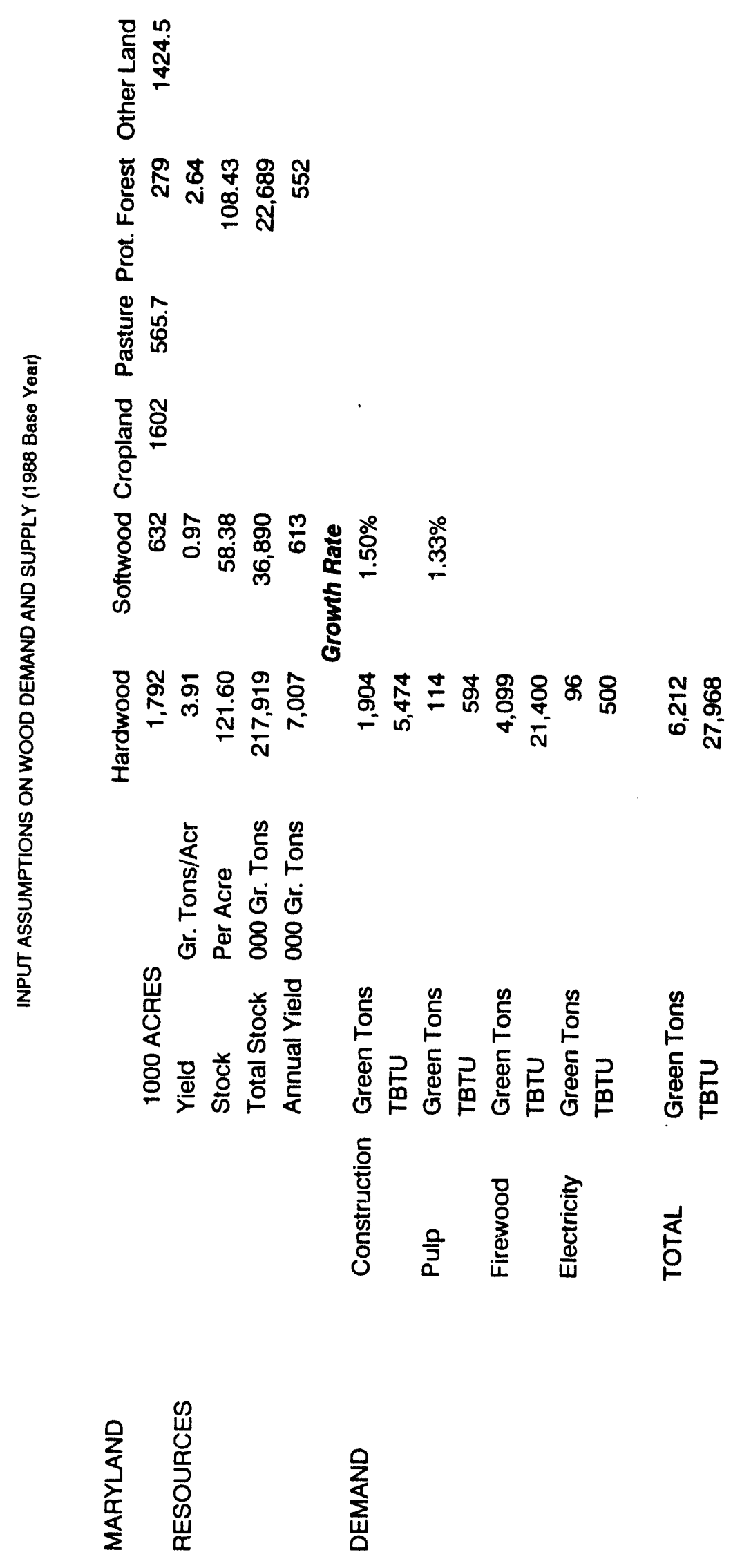




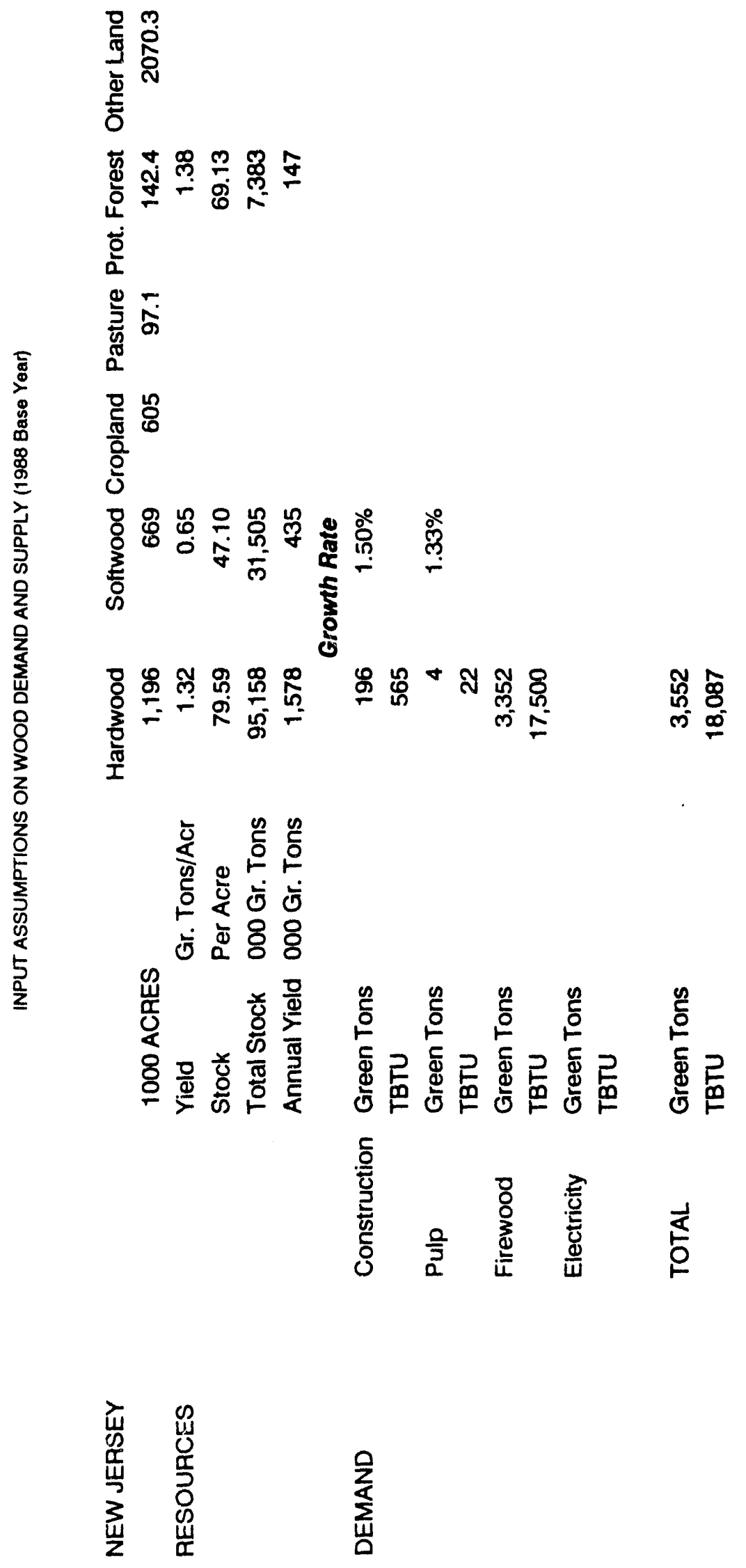




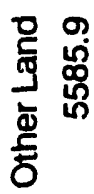

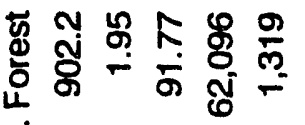

ธิํㅁ

莺

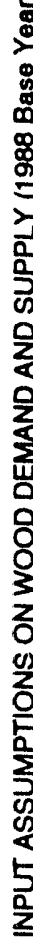

尊尽

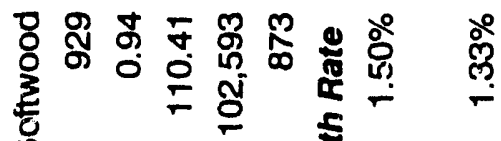

ईิ

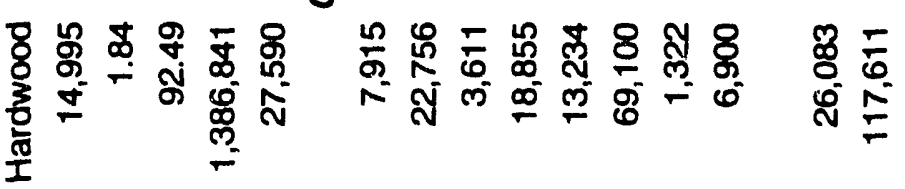

这 $\stackrel{n}{0} \stackrel{0}{0}$

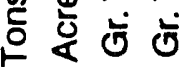

迥 ๕

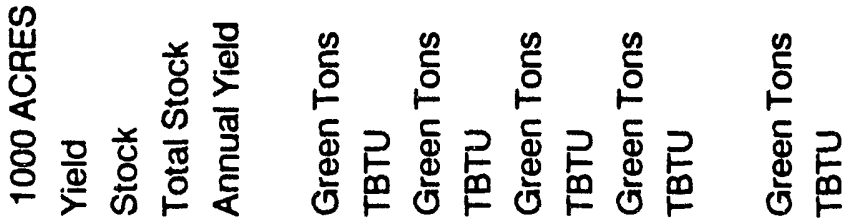

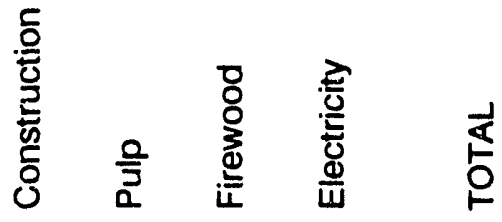

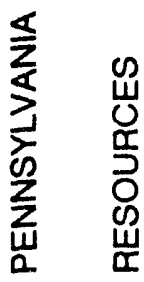

$\sum_{\text {蒠 }}^{0}$ 


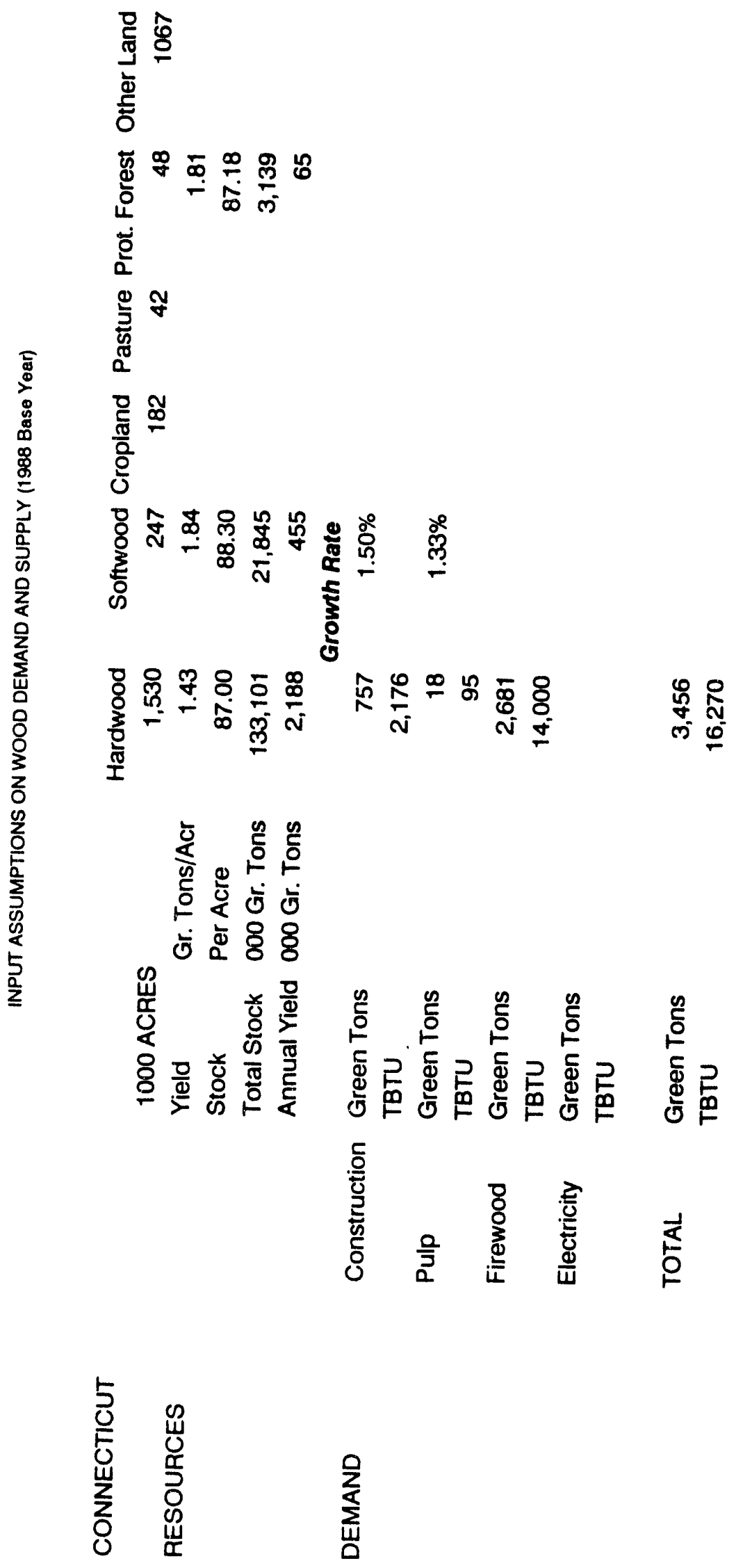


苗

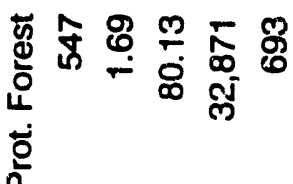

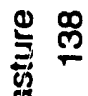

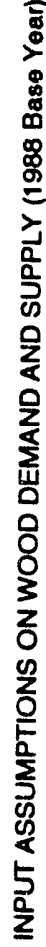

융

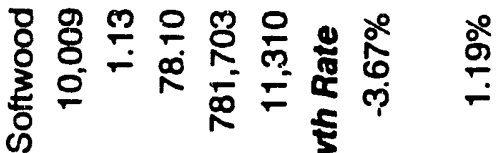

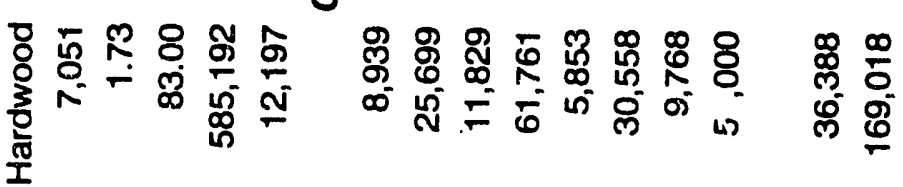

눙 능 응

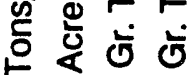

ज通

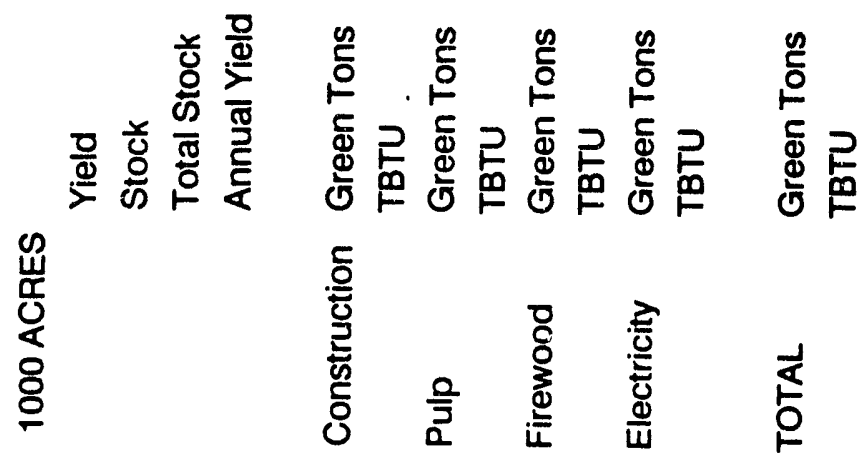

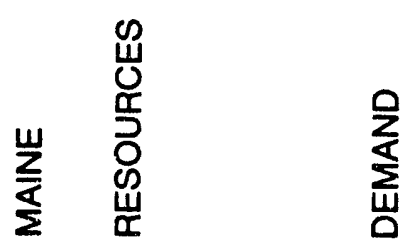




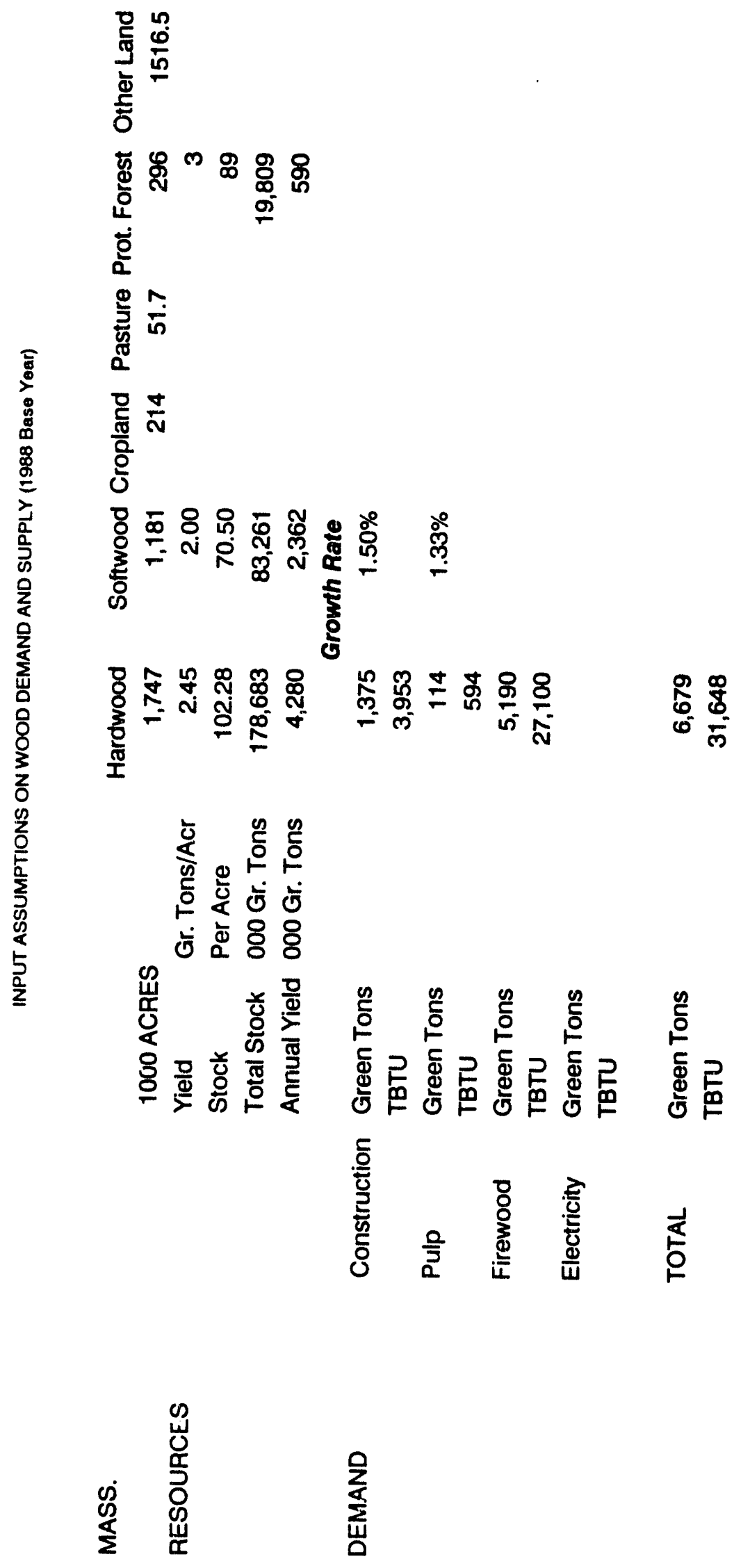




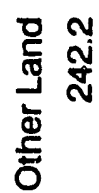

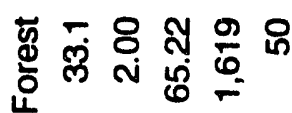

ธั่

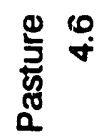

듀

응

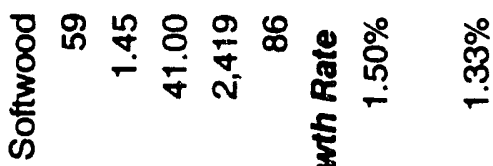

ปั

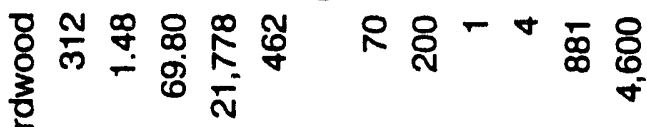

సू

I

递客

氞造通

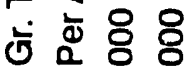

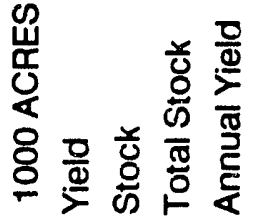

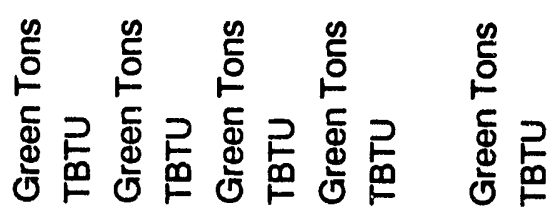

总

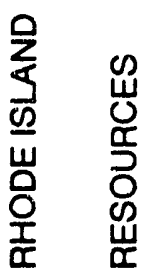

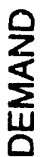




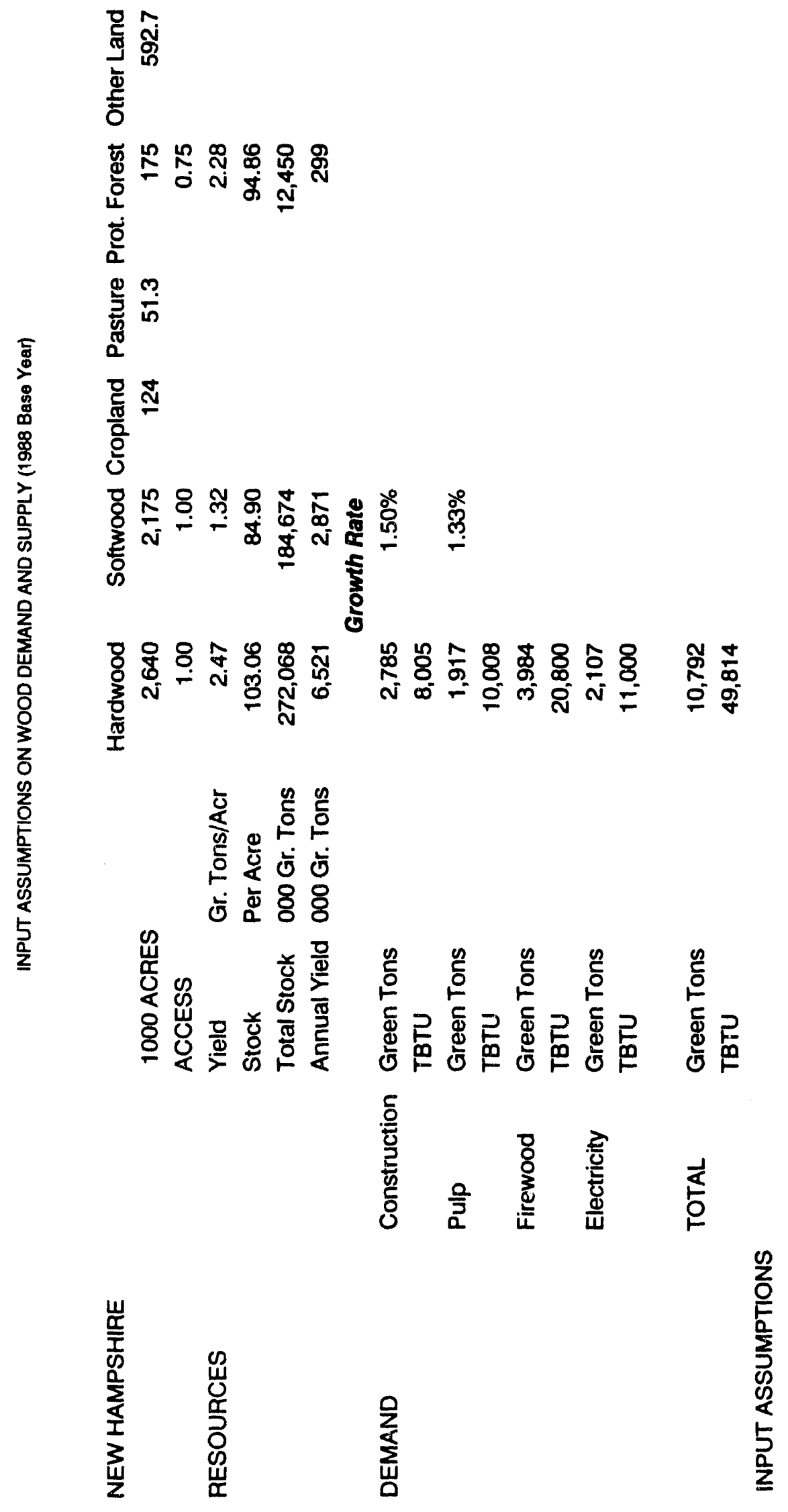


苞

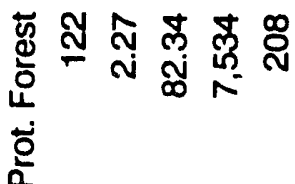

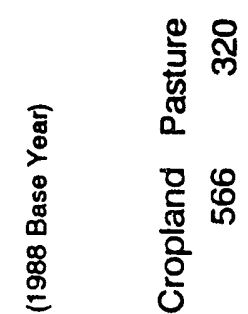

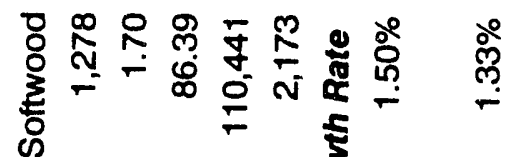

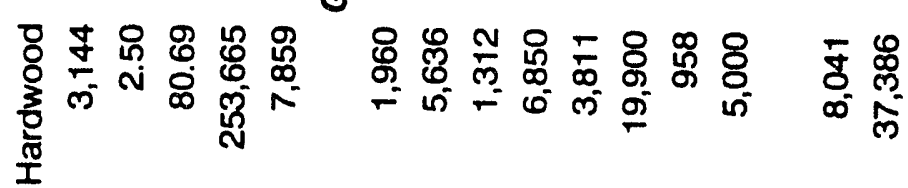

这

号过过

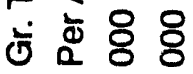

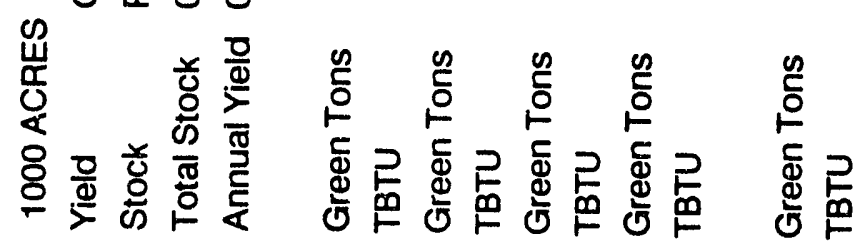

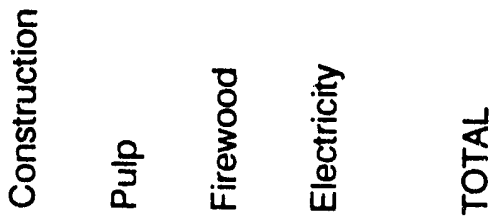

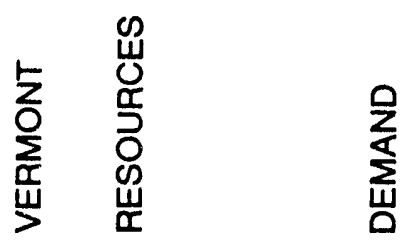




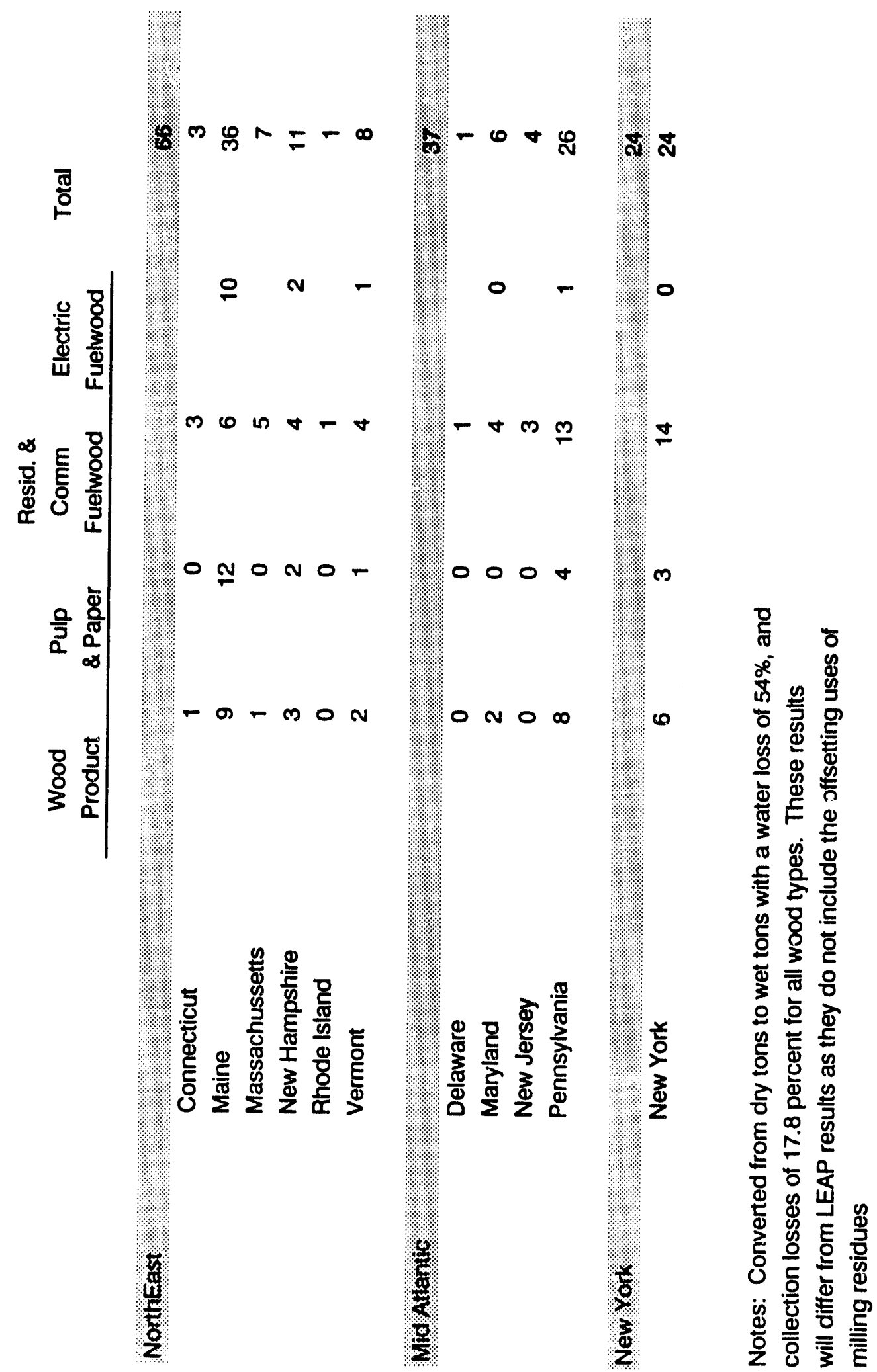




\section{APPENDIX C \\ SUMMARY DESCRIPTIONS OF CONEG STATE FORESTS}

AND FOREST PRODUCTS

\section{New England}

\section{Connecticut}

About fifty nine percent of Connecticut is covered by some 1.8 million acres of forest land, almost entirely available to commercial uses. Small private landuinners about 92,000 individuals - hold about 1.5 million acres or 88 percent of Connecticut's forest resources; state-owned tracts make up the remaining 12 percent.

Connecticut is a densely populated state, and despite the high percentage of its land in forests, there is only about an acre of land in total for every resident of the state. The state estimates some 3 thousand acres a year are lost to development. Recent legislation in the form of a "giobal warming bill" takes definite steps to miligate the effects on the environment from forest cover destruction for the sake of development. About 500 of the 92,000 private owners have enrolled in the American Tree Farm System and the total acreage of their certified tree farms equals about 150 thousand acres.

About 86 percent of Connecticut's commercial forests are hardwood. The oakhickory forest type is the most common species group in the Connecticut forest but red maple is the most prevalent individual species across the state in terms of growing stock volume. The northern hardwoods, including maples, birch and beech account for about a quarter of the state's forested area.

Connecticut's forest is showing clear indications of maturity. Between the last two U.S. Forest Service inventories of the state's forest resource, the volum:e in the sawtimber size trees increased by 44 percent. The sawtimber growth doubled during the interval between surveys, and this was the fastest gain in Southern New England. Sawtimber stands make up nearly two-thirds of the state's forested area. Likewise, there is less area taken up by seedlings and saplings than there was 20 years ago.

Connecticut contributes a very small amount to the region's paper and pulp production. Although Connecticut has a relatively small share (5.6 percent) of New England's timber, and less than 5 percent of its wood products production, it has an above average share of the region's value-added related to wood products manufacturing. Connecticut's species and products are in high demand in Europe and 
the Asian countries. Wood exports from Connecticut increased 50 percent in 1987 alone.

Per capita wood use for end-use energy in Connecticut is about 4.5 MMBtu, comparable to that in Massachusetts and Rhode Island, but much lower than Vermont, New Hampshire and Maine, where per capita firewood use is several times higher (about 20 to 40 MMBtu per capita).

\section{$\underline{\text { Maine }}$}

With almost 18 million acres of forested land, about 89 percent of its land area, Maine is the most heavily forested state in the country. Ninety-seven percent of Maine's forest land is available for commercial exploitation; only 3 percent is protected. Virtually all of the commercial timberland in Maine is privately owned, about half by forest industry and only half in smaller private holdings; this is unlike the pattern throughout the other CONEG states where small private holdings dominate.

Maine has the highest percentage of the forest industry's forest land in the U.S.no other state has as much corporate ownership. This strong focus on industrially owned and managed forest resources is one reason why, in relation to the other 10 CONEG states, there is so much intensive management. Much of the timberland is held to produce income on a sustained basis, or to provide a certain amount of fiber to specific pulp and paper mills or sawmills in Maine or eastern Quebec.

Maine's extensive commercial forest in the northern part of the state is still recovering from the after-shock of a massive epidemic of the spruce budworm in the late 1970s. Millions of acres of spruce-fir forest were severely defoliated, mortality was extensive and the landowners were, for the most part, forced to harvest aggressively to recoup these damaged trees before they were no longer salvageable. The result was a severe disruption in the balanced distribution of age classes within the spruce fir forest. Consequently there is concern about the availability of sawtimber sized trees early in the next century. In response to that situation many major landowners are actively pursuing proven techniques (planting, herbicide brush control and density or spacing control) to shorten the period it takes to bring young stands to maturity. Regardless of the budworm, Maine's softwood stands tend to regenerate profusely and growth becomes inhibited within $20-25$ years or so unless something is done to reduce the number of stems/acre.

Although Maine is regarded by many as a softwood state (its softwood acreage is second only to Georgia on the east coast) the Maine forest is actually 40 percent hardwood. The northern hardwoods - birch, beech, and maple, as well as oak and ash, are significant and valuable parts of Maine's forests. World deinand for these hardwoods is increasing dramatically as major industrialized countries in Europe and Asia react to the growing concern over the depletion of tropical hardwood forests. 
Some major landowners are increasing their hardwood management activities because of these increasing values. Hardwood stands were frequently neglected during the past 15 years as budworm salvage operations took top priority. There is also a wide variety of markets for various wood products in Maine itself, including end-use and electric power supply fuels. With the exception of the extreme northern areas of the state, markets are relatively well distributed.

There is a concern that Maine's forests are being harvested faster than they are being replenished in order to satisfy the increasing demand for fiber (Forests, 1988). The recent Northern Forest Lands Study (USDA-FS, April 1990) examined many of these issues. A variety of views were expressed during the study including many from the forest industry who argued that the current harvesting is removing slow growing or over-mature stands and turning those sites into more vigorous stands. They argued that with intensive management techniques fewer acres of timberland could provide the same amount of product as today. Alternatively, many environmentalists argued that more intensive management would harm biodiversity and limit recreational opportunities.

Despite the preponderance of private ownership, forest management is fairly heavily regulated - through zoning and other statutory procedures. The Land Use Regulation Commission (LURC) has comprehensive zoning authority over many of the activities in the state's 10 million acres of unorganized territory. This authority relates mostly to road building, shoreland development and erosion control, and protection of critical areas. A Forest Practices Act regulates timber harvesting statewide - especially clear-cutting. Professional foresters must be licensed to manage timberland, and the industry has a voluntary certification program for professional loggers which attests to the successful completion of specialized training in silviculture, business practices, harvesting techniques and safety.

Maine contributes almost 80 percent of New England's paper and pulp production (and half of the entire CONEG region production), 56 percent of its wood products, 26 percent of its end-use energy consumption, and 76 percent of its wood for electric power supply (two-thirds of the CONEG region total). Per capita end-use wood energy consumption in Maine - about 27 MMBtu - is far above that of all other CONEG states except for Vermont and New 'Hampshire.

Wood energy markets play a significant role in the integrated management of Maine's woodlands. Whole tree chipping for pulp and energy of poorly formed and defective trees, as well as the recovery of harvesting residues, has enabled foresters to upgrade the quality of forest stands, especially in hardwood or mixed stands. Seven free-standing power plants and numerous cogenerating facilities across the state have been a major influence on industrial and non-industrial timberland management practices. 


\section{Massachusetts}

Massachusetts is about 64 percent forested, with approximately 3 million acres of forest land. The forest industry owns only about 2 percent of Massachusetts commercial forest. Small private holdings account for 2432 thousand acres or 83 percent, while the state owns about 430 thousand acres or 15 percent. Many areas in Western Massachusetts have an even higher percentage of forest.

Like much of the rest of New England, the Massachusetts forest are mature the old abandoned farms that gradually regenerated back to trees are now coming of . age and could be harvested. About 60 percent of the state's unprotected forests are hardwood. Forest Service surveys indicate a substantial 57 percent increase in sawtimber volume statewide between 1972 and 1986. The growth in white pine during that period centered on larger trees above 13" in diameter, and at the same time the volume in smaller diameter white pine decreased by 12 percent. White pine is still the most prevalent species within the Massachusetts forest in terms of volume - it makes up about 25 percent of the standing volume statewide. Red maple is the second most prevalent, followed by red oak which accounts for 12 percent of the total volume of growing stock.

Although Massachusetts has a substantial and valuable timber base, there are a number of constraints affecting its utilization. First, most of the timberland is held by some 235,000 individual landowners, and the majority of these ownerships are less than 10 acres in size. Although there is a current use tax system for managed woodlots greater than 10 acres in size, only 7 percent of the eligible acreage is enrolled in this tax relief program. Much of the timber owned by former urbanites will be difficult to bring under long-term management plans as these owners tend to prefer preservation to management. Second, the small scale of the typical harvesting operation in Massachusetts makes it difficult to absorb the high overhead costs of complying with state and various local harvesting ordinances. This may account for the fact that Massachusetts grows about three times as much as it harvests each year with respect to all products.

Overall wood production and use in the state is generally lower than elsewhere in the New England region. Massachusetts is responsible for about 9 percent of New England's wood products production, less than 1 percent of its paper and pulp production, and essentially no wood electric power generation. However, Massachusetts does consume a large share of the New England region's firewood, owing primarily to its relatively large population; its per capita consumption is about 5.74 MMBtu. 


\section{New Hampshire}

New Hampshire has 5.0 million acres of forest land, about 87 percent of total land area in the state, making it the second most forested state in the nation. Commercially exploitable timberland comprises about 96 percent of the total forest land, or 4.8 million acres. The large forest industry owns about 657 thousand acres or about 14 percent of commercial timberland, public forests account for 14 percent, and small holders account for about 3.5 million or about 70 percent of the total.

About 55 percent of the total commercial timberland in the state is hardwood. White pine comp: es the greatest percentage of the softwood timberland, making this species commercially the most important to the state's forest industry. New Hampshire has a strong forest industry which purchases an estimated 80 percent of the timber harvested annually within the state.

There is considerably less, on a total or percentage basis, of the spruce-fir type which is common in Maine, but white pine, a much more valuable species for commercial wood, predominates in most of the state. Also, there is nearly three times as much hardwood in New Hampshire as there is spruce-fir. The annual harvests are dominated by softwoods, however, and as much as 90 percent of the annual softwood growth (mostly white pire) is cut each year. Canadian and Vermont sawmills buy much of the hardwood and softwood logs which are harvested from New Hampshire's forests. Much of the state's former wood manufacturing base has relocated or gone out of business and landowners who want to sell their timber have a limited selection of markets within the state. Unfortunately, when logs are transported great distances to these out-of-state mills the landowners stumpage return is reduced since much of the value of the delivered product is sacrificed to the cost of transportation.

Approximately one-third of New Hampshire's harvest comes from timberlands in Coos County, the northernmost county of the state where a large percentage of the forest is owned by industry. About 80,000 small landowners own the rest of the private land, but 55,000 of these owners have less than 10 acres in their possession. Those owners with more than 50 acres control about 80 percent of the small ownership acreage. Unlike in Maine, some of the best timberland in the state (especially for hardwoods) is in the White Mountain National Forest. This forest land is generally inaccessible due to wilderness set-asides. New Hampshire has some 800 thousand acres certified by the American Tree Farm Program.

Wood energy is a significant force in New Hampshire's forest management systems. The state has about 130 megawatts of wood-fired electric capacity, which requires about 2 million tons of chips per year - an amount equivalent to about 30 percent of the state's total annual harvest. The state regards this market as an opportunity to encourage forest management on small woodlots. The state consumes 16 percent of New England's wood for electric power supply. New Hampshire also uses 
about 18 percent of the total end-use firewood used in New England, with a high per capita consumption at about $23 \mathrm{MMBtu}$.

\section{Rhode Isiand}

Rhode Island has one of the smallest forest resource bases of the 11 CONEG states: approximately 400 thousand acres. Despite this relatively small absolute figure, about 59 percent of the state's total land is forest. Virtually all of this forest land is in small private holdings. Ninety percent of Rhode Island's forest resources are owned by 14,000 private, non-industrial owners in parcels ranging from one to five hundred acres. A census taken in 1977 showed thai 2,000 of these owners have cut trees from their property. In that same year Rhode Island ranked 46th in the volume of timber harvested in the continental United States.

Unlike other New England states to the north, the Rhode Island forest was actually dwindling in size during the 20-year period from 1950-1970. Although 8 percent of Rhode Island's timber base was lost to development during that period (at a time when some neighboring states saw their forested acreage actually increasing as old farms reverted to forests), this trend has stopped and the state remains about 60 percent forested - on par with Connecticut.

What Rhode Island's forest land lacks in size it makes up for with quality. The forest in this state is about 84 percent hardwood and much of it is valuable red and white oak, used for furniture and other commercial products. In the softwood component white pine makes up nearly two-thirds of the growing stock and nearly three-quarters of the sawtimber volume. Very little of Rhode Island's timbered acreage is not in production -98 percent is classified as commercial timberland. Rhode Island's distance from major wood products manufacturing centers makes it difficult to manage small tracts of timber for any kind of profit.

Rhode Island contributes only 0.4 percent of New England's wood products production and no paper and pulp. Its 4 percent of the region's end-use fuelwood consumption is based on about 4.8 MMBtu per capita.

\section{Vermont}

Vermont is 77 percent forested with about 4.5 million acres of timberland. The forest industry owns only about 9 percent of Vermont's timberland, and nearly all of that acreage is located in the "Northeast Kingdom" in the northeastern corner of the state. These owners export much of their annual harvest to Quebec, New Hampshire, and Maine. Small, non-industrial private owners own about 3.5 million acres, or about 80 percent of total forested land. This group also owns about 80 percent of the most valuable sawtimber acreage in the state. The Green Mountain National Forest, together with state forests, comprise about 10 percent of Vermont's forest but it also 
has the highest percentage of acreage with above average stocking of sawtimber volumes.

At one time Vermont was over 80 percent open farmland; much of today's forest derives from old fields which were abandoned, grew back into a forest, and is now maturing. About 70 percent of Vermont's forest is hardwood. Vermont's forest is nearly one quarter sugar maple; this species "accounts for 23 percent of the growingstock volume and 24 percent of the sawtimber volume" (USDA-FS, September 1987). The northern hardwoods as a group make up about 60 percent of the forest cover approximately four times the area that grows predominantly spruce and fir. White pine is an extremely valuable part of the state's softwood resource but only occupies about 10 percent of the forest.

Although Vermont's forest has a high percentage of high grade sawtimber volumes, the annual harvest still has a higher share of lower grade products such as pulpwood and fuelwood. In 1982, for example, the amount of wood cut for fuel was nearly 40 percent of the total harvest, according to the U.S. Forest Service decennial survey (USDA-FS NE-87). Fuel wood from Vermont provides energy to the Burlington Electric power plant as well as plants in New Hampshire.

Even though Vermont's secondary manufacturing facilities use Vermont lumber for three-quarters of their raw material needs, the state still imports a fair amount of raw material from neighboring states. In 1982, the last year a census was taken, the industry claimed it actually imported more sawlogs than it exported. Many of Vermont's sawmills are located near the boundaries with New York and New Hampshire, faciliting such imports (USDA-FS, September 1987), and the interstate highway facilitates exchanges with Quebec. Overall, Vermont is growing much more wood than it harvests, but in the Northeast Kingdom there is some concern that nearly all or most of the growth is harvested on an annual basis (Stone, 1991).

The Burlington Electric power plant has had a positive effect on forest policy in Vermont. Concerns about clear-cutting and the potential for adverse management practices, that whole tree chipping entail, caused the state to require that harvesting be done by licensed operators and their work would be monitored on a regularly scheduled basis. This encouraged "best management practice" and stimulated workshops for loggers in the state. It established the practice of linking the purchase of wood to the means by which it was harvested.

Still, clear-cutting and the possible loss of biodiversity in the wake of such harvests remains a concern to environmentalists (e.g., in the Sierra Club and Audubon Society) and some state officials. The Department of Public Service believes, on the other hand, that ten years of data on this practice shows no evidence of biodiversity loss. The issue is probably the size of clear-cuts, rather than the actual use of clearcutting as a management tool. Some foresters feel that the quality of clear-cut 
operations (i.e. erosion control, wildlife buffers, etc.) is superior on the mechanized chipping operations compared to the more conventional skidder (chainsaw logging operation).

Vermont contributes about 10 percent of New England's wood products and paper and pulp production. It consumes about 17 percent of New England's end-use wood energy, with a per capita consumption of about $39 \mathrm{MMBtu}$, far above the region's average of 9.6 MMBtu. Finally, Vermont uses about 7.5 percent of the region's wood for electric power generation.

\section{Mid-Atlantic}

\section{Delaware}

Delaware has the least amount of forested acreage of all the states in the

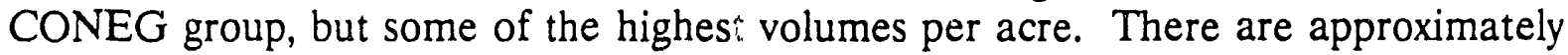
390 thousand acres of timberland, about 33 percent of the total area in the state. Most of this acreage, 97 percent, is commercial timberland. Overall, about 88 percent of the state's commercial timberland is in non-industrial holdings - private individuals own approximately 330 thousand acres. There are 17,000 separate private ownerships, and 11,000 of these are less than 10 acres in size. There is an average of 103 tons of live trees/acre and 4,748 board feet/acre of sawtimber. In comparison, the state of Maine has about 86 tons/acre of live trees and 2,795 board feet of sawtimber/acre, on the average.

Although one-half of Delaware is agricultural land, over 7 thousand acres of timberland is harvested each year. The forest industry, primarily two paper companies, owr about 8 percent of the state's timberland. About 4 thousand acres of this total is in the hardwood forest in the northern half of the state. Some 3 thousand acres in the soluthern state are cut annually. On these sites loblolly pine is intensively managed on shurt rotations (20 years) for pulpwood. Many of these operations are clear-cut, followed by intensive site preparation and followed by herbicide spraying for control of hardwood competition. There are 110 tree farms certified by the American Tree Farm Program with a total of 40 thousand acres.

Oak/hickory is the major forest type, but loblolly pine is the major species across the state. Dover is about the northernmost reach of this southern pine. The U.S. Forest Service inventory registered an 11 percent decline in the loblolly growing stock between 1972 and 1986 as well as a slight increase overall in the rate of removals versus annual growth in the softwoods in general. The same inventory indicated a 12 percent increase in the area occupied by sawtimber stands along with a corresponding decrease in the area occupied b" seedlings and saplings. These are likely indications of 
a maturing forest which may be past its most rapid growth and rate of carbon sequestration.

Delaware contributes relatively little to the Mid-Atlantic region's wood product production and wood-energy usage. It produces about 2 percent of the region's paper and pulp and wood products, uses 4 percent of its end-use wood energy (with a per capita consumption of about $7 \mathrm{MMBtu}$ ), and no wood for electricity.

\section{Maryland}

There are about 2.7 million acres of forest in Maryland, or 43 percent of the state's total land area. Ninety percent of this resource is classified as commercial timberland, and 85 percent of this is held by non-industrial private owners. These 2 million acres are owned by 95,800 individuals, with an average holding of about 24 acres in size. This average ranges from a little less than 16 acres near urban areas in the central part of the state to 50 acres in the less populated counties in extreme western part of the state. Three major paper companies own the majority of the 130 thousand acres of the industrial portion of private lands.

The industrial forest owners are most active in the areas known as the Lower Eastern Shore and Western Maryland. Although the industrial ownership is only 6 percent of the total forest, the influence of industrial style management extends to other acres through company sponsored landowner assistance programs. As is the case in most CONEG states, reaching the small private owner is still a challenge since over. half of these owners have less than 10 acres. Maryland lost about 16 percent of its forest land over the past 20 years as more valuable uses for these acres were found. The state recognizes the threat these development opportunities pose to the future of the resource and the Forest Conservation Act monitors and mitigates the loss of forest cover, site by site.

Maryland's forests extend from the Atlantic ocean in the east to the Appalachian Region in the west. The diverse soils and drainage patterns across these regions yield a diversity of forest types. On the Eastern Shore much of the intensive forest management takes place when poor hardwoods are converted to loblolly pine stands. Although some of these sites are highly regulated through environmental controls to protect the quality of Chesapeake Bay, it is still feasible to make the investments in superior planting stock and site preparation costs which loblolly pine management demands. These cultural activities are conducted by major paper companies which own nearly 25 perceni of the four counties in the Lower Eastern Shore. This area is just about the northernmost range of loblolly pine.

Further west, in the Piedmont Province, the soils change and the forest species mix shifts towards hardwoods like oak and tulip poplar. In the Allegheny Plateau near Pennsylvania and West Virginia the typical northern hardwoods are found as well as 
red and white oak. Yellow poplar is the number one species statewide and it represents 12 percent of the growing stock volume. Maryland is basically a hardwood state - over 80 percent of the resource is made ur of oak, poplar, and gum; the remaining 20 percent is accounted for by loblolly and Virginia pines. Maryland is a "crossroads" for some species which are at the southernmost or northernmost extent of their ranges.

Although growth exceeded harvest on a statewide basis according to latest U.S. Forest Service survey in 1986, the removals exceeded growth on the Lower Eastern Shore. Most of the sawtimber that is cut in Maryland remains in-state for primary manufacturing. Oaks represent a third of the annual sawtimber harvest and a like amount is in loblolly pine.

Maryland uses about 19 percent of the Mid-Atlantic region's total end-use fuelwood consumption (with a per capita consumption of about $5 \mathrm{MMBtu}$ ), produces about 19 percent of its wood products, and uses about 7 percent of the wood for electricity generation in the region.

\section{$\underline{\text { New Jersey }}$}

New Jersey's total land base is 42 percent forested for a total of 2 million acres. There are no industrial ownerships, and some 64,000 private individuals own 75 percent of the state's commercial forest land. The balance is public land. About 3,000 private woodland owners - with about 116 thousand acres - participate in the state's Farmland Assessment Program, and have their forest land properties under professional management. There are 228 tree farms totaling 84 thousand acres certified under the American Tree Farm system.

Oak-hickory is the predominant forest type in New Jersey, but red maple is the most common single hardwood species, with 12 percent of the state's growing stock. volume. Pitch pine is the most prevalent softwood species and it has 14 percent of the state's growing stock volume. New Jersey harvests less than 25 percent of its annual growth each year, and only about 13 percent of its annual growth in sawtimber. Most of the sawlog harvest is accounted for by valuable hardwood species such as ash, yellow poplar, and red and white oaks, and chestnut oak.

Although New Jersey's primary wood manufacturing industry (sawmills) is small in comparison to its extensive industrial base, the state has a strong secondary manufacturing base which uses New Jersey products but also relies on the import of lumber from other states. A comprehensive wood energy program for the state needs to deal with the manufacturing residues from these industries.

The fact that New Jersey, one of the most densely populated, urban states in the Union, is still nearly half covered with forests, certainly attests to the tenacity and 
renewability of the Northeastern forests. A most striking contrast to the industrialized sectors of the Northeast is the unique pinelands of South Jersey. The "Pinelands Protection Act" entails a "Comprehensive Management Plan", and the Act states: "The legislature hereby finds that the pinelands area comprises pine-oak forests, cedar swamps, and extensive surface and ground water resources of high quality which provide a unique habitat for a wide diversity of rare, threatened, and endangered plant and animal species...".

New Jersey contributes about 16 percent of the Mid-Atlantic region's total enduse fuelwood consumption (with the lowest per capita use in the entire CONEG region, at about $2.4 \mathrm{MMBtu}$ ), and about 2 percent of its total wood products.

\section{Pennsylvania}

Pennsylvania is a highly industrialized state, with a significant agricultural sector, a large population, and substantial amounts of timberland. In essence, it has something in common with nearly each of the other states and in the same order of magnitude. For example, it has nearly as much timberland as Maine, but its industrialization is much greater and has more in common with New Jersey. Four broad topographical divisions give Pennsylvania a little bit of what each of the other states has in terms of forest type - the land base stretches from a coastal plain and piedmont region in the southeast corner, across the ridge and valley section of the Appalachian Mountains, which run from Maryland northeast to New Jersey, and then a plateau region in the north and west sections of the state. Although the oak-hickory type is most common ( 7.5 million acres, 47 percent of the forest area), the northern hardwoods are close behind (6.3 million acres, 40 percent of the area).

There are 16.8 million acres of forest land in Pennsylvania, about 58 percent of its land base. Almost all is classified as conmercial timberland. Most of the commercial timberland - about 80 percent or 12.5 million acres - is privately owned, and only 8 percent of this is in industrial holdings. There are nearly one-half million private ownerships. Three hundred thousand of these owners have one million acres in parcels less than 10 acres, but a large amount of private land, almost 4 million acres, is in ownership with more than 100 acres. There are over 3 million acres of public forest, and this includes the Allegheny National Forest.

Pennsylvania is essentially a hardwood producing state. Over 90 percent of the resource is hardwood species, by volume. Various oaks account for 34 percent of the total timber volume and nearly 40 percent of the Commonwearth's sawtimber volume. The maples make up 25 percent of the total volume and nearly 20 percent of the sawtimber volume. Valuable black cherry is about 9 percent of the total volume and 10 percent of the sawtimber volume. 
The net growth in volume exceeds the volume annually harvested by 2 to 1 . Although sawtimber stands have increased steadily in recent years (the continuing after effect of extensive harvesting near the turn of the century), there is concern about the quality and value of this sawtimber resource. A Forest Productivity Report completed in 1979 identified opportunities to increase productivity on 1.8 million acres, across all categories of ownerships, through commercial thinning. It might be difficult to justify these efforts in a state where there is a surplus of timber and alternative uses for the land.

The overwhelming proportion of the Mid-Atlantic region's wood products and wood energy use - 98 percent of its paper and pulp, 77 percent of its wood products, 62 percent of its end-use fuelwood, and 93 percent of its wood-electric energy generation occurs in Pennsylvania. End-use wood energy consumption is about 5.8 MMBtu per capita.

\section{New York}

There are 18.5 million acres of forest land in New York state, about 61 percent of its total land area. About 3 million acres are in "preserved" lands such as the Adirondack Park, and the balance is classified as commercial timberland. Only 7 percent of the state's 15.4 million acres of commercial timberland is in relatively large industrial holdings; the bulk is held by other private owners.

New York is predominantly a hardwood state, with 84 percent of its commerciui forest having a plurality of hardwocd species; 20 percent of the nation's sugar maple inventory is within New York state. Also, black cherry, a valuable wood products resource, grows in the southwestern part of the state, near Pennsylvania.

During the 1970s New York registered an increase of nearly 1 million acres of timberland, as old farmland which reverted to trees became fully stocked. Although New York grows nearly twice as much as it harvests on a statewide level, the cut and growth are nearly the same in the southwestern tier where forest management and utilization is more intense. Much of the state's forest is mature with large diameter sawtimber.

The nature of the local industries and the quantity and quality of the products harvested go hand in hand. For example, many of New York's pulp and paper mills are located in the Adirondack region because of the abundant timber and water: over 70 percent of the state's pulpwood harvest is generated in this "northern tier". Likewise, over one-half of the sawlogs harvested are in the southwestern region where the state's best sawtimber and most valuable species are located.

About half of the lumber which New York produces is considered high grade and 40 percent goes on to become furniture. Although many of New York's hardwood 
sawmills are nearby, New York is a net exporter of sawtimber and Pennsylvania is New York's biggest trading partner in this respect. The interstate highway system also gives New York ready access to lucrative sawtimber markets in Canada; one study estimated New York loses 1,600 jobs, $\$ 10$ million in payroll and a potential $\$ 19$ million in value. added to Canada each year through the state's export of valuable sawtimber. Despite the fact that many New York companies need lumber for their secondary manufacturing operations, over half of the state's lumber production is shipped to other states.

Any program aimed at influencing the nature or intensity of forest management and forest conservation on New York's vast commercial forest is hampered by the extremely fragmented nature of the ownership. There are over 500 thousand separate private ownerships that control 94 percent of the state's timberland. As land is sold and resold, long-range forest management plans become diluted or lost forever. Half of these small land holdings are less than 10 acres in size, and there are only 600 ownerships with 1000 or more acres of forest. The forest industry owns less than 9 percent of the resource.

New York contributes about 17 percent to the CONEG region's total wood products and pulp output, and about one-quarter of its residential and commercial fuelwood consumption. It does not as yet have a significant amount of wood-electric power supply, at less than 2 percent. 

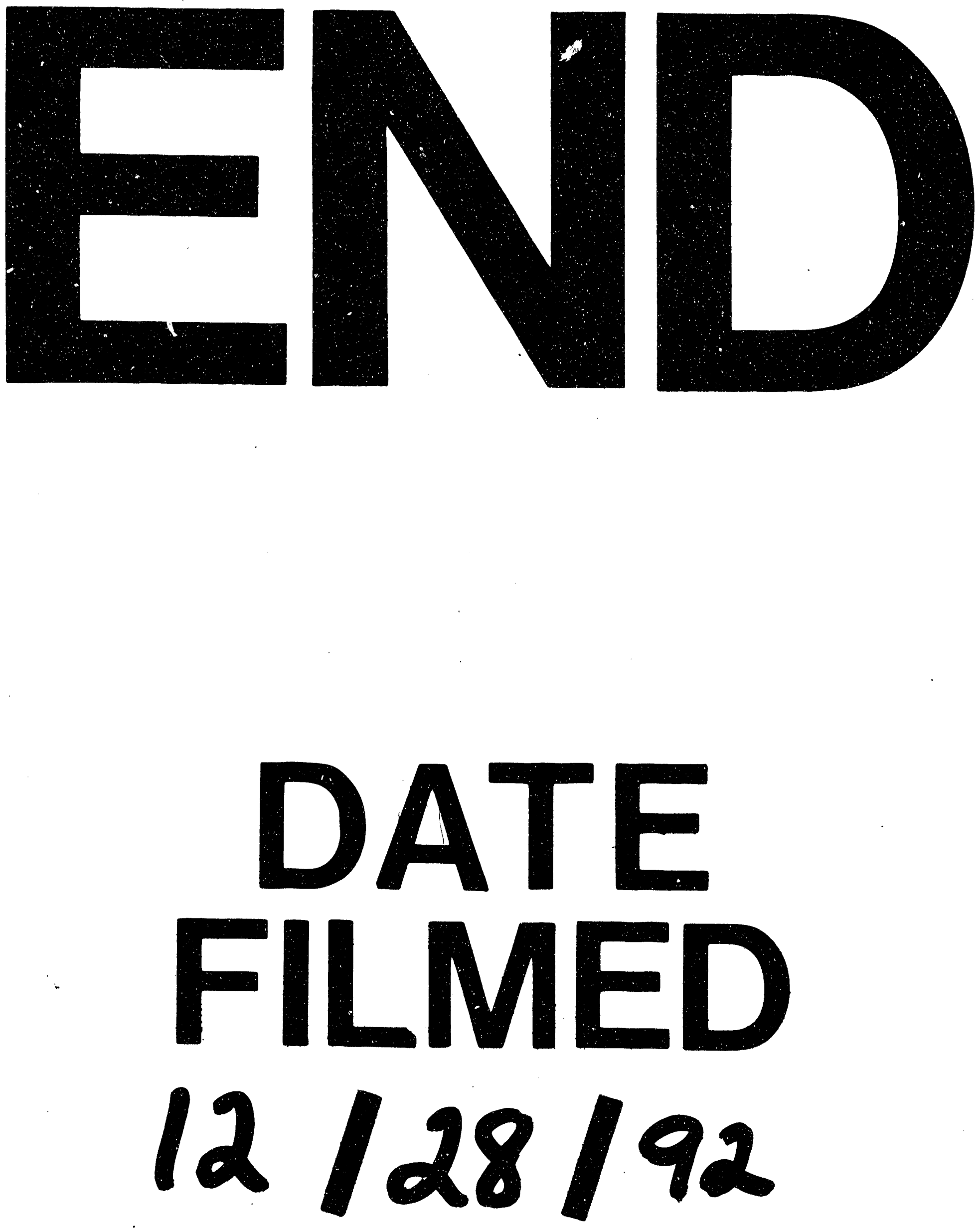
